\title{
MASTER
}

\section{Projections of Spent Fuel to be Discharged by the U.S. Nuclear Power Industry}
C. W. Alexander
C. W. Kee
A. G. Croff
J. O. Blomeke

\section{OAK RIDGE NATIONAL LABORATORY}




\section{Printed in the United States of America. Available from National Technical Information Service \\ U.S. Department of Commerce 5285 Port Royal Road, Springfield, Virginia 22161 \\ Price: Printed Copy $\$ 6.00$; Microfiche $\$ 3.00$}

This report was prepared as an account of work sponsored by the United States Government. Neither the United States nor the Energy Research and Development Administration/United States Nuclear Regulatory Commission, nor any of their employees, nor any of their contractors, subcontractors, or their employees, makes any warranty, express or implied, or assumes any legal liability or responsibility for the accuracy, completeness or usefulness of any information, apparatus, product or process disclosed, or represents that its use would not infringe privately owned rights. 


\section{DISCLAIMER}

This report was prepared as an account of work sponsored by an agency of the United States Government. Neither the United States Government nor any agency Thereof, nor any of their employees, makes any warranty, express or implied, or assumes any legal liability or responsibility for the accuracy, completeness, or usefulness of any information, apparatus, product, or process disclosed, or represents that its use would not infringe privately owned rights. Reference herein to any specific commercial product, process, or service by trade name, trademark, manufacturer, or otherwise does not necessarily constitute or imply its endorsement, recommendation, or favoring by the United States Government or any agency thereof. The views and opinions of authors expressed herein do not necessarily state or reflect those of the United States Government or any agency thereof. 


\section{DISCLAIMER}

Portions of this document may be illegible in electronic image products. Images are produced from the best available original document. 
ORNL/TM-6008

Contract No. W-7405-eng-26

CHEMICAL TECHNOLOGY DIVISION

PROJECTIONS OF SPENT FUEL TO BE DISCHARGED BY THE

U.S. NUCLEAR POWER INDUSTRY

C. W. Alexander

C. W. Kee

A. G. Croff

J. 0. Blomeke

Date Published: October 1977

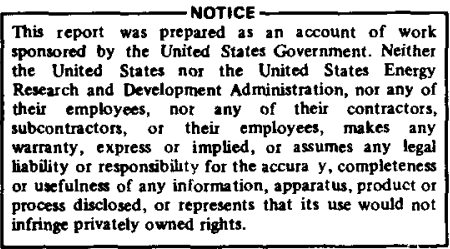

OAK RIDGE NATIONAL LABORATORY

Oak Ridge, Tennessee 37830

operated by

UNION CARBIDE CORPORATION

for the

DEPARTMENT OF ENERGY 
Abstract ........................... 1

1. Introduction . . . . . . . . . . . . . . . . . 1

2. Reactor and Fuel Characteristics .............. 2

3. Projections of Spent Fuel to Be Discharged . . . . . . . . . 3

4. NAC Projections . . . . . . . . . . . . . . 6

5. References ........................ . 7 
PROJECTIONS OF SPENT FUEL TO BE DISCHARGED BY THE U.S. NUCLEAR POWER INDUSTRY

C. W. Alexander, C. W. Kee, A. G. Croff, and J. O. Blomeke

\section{ABSTRACT}

Calculated properties of spent fuel projected to be discharged and accumulated by the U.S. nuclear power industry through the year 2031 A.D. are presented. The projections are based on installed nuclear capacities of 380 and $543 \mathrm{GW}(\mathrm{e})$ in the year 2000 and 2030, respectively. They include compilations of the grams of the elements, curies of radioactivity, thermal decay power, photon and neutron emission rates, and radiotoxicities of the assemblies that are accumulated at a Spent Unreprocessed Fuel Facility (SURFF), allowing for delays of 5 and 10 years before shipment to SURFF.

\section{INTRODUCTION}

This report summarizes the quantities and properties of spent fuel projected to be discharged from LWRs in the United States through the year 2031 A.D. The calculations are for use in the planning and design of the Spent Unreprocessed Fuel Facility (SURFF), which may be used to store the fuel pending a decision as to its final disposition.

Table 1.1 presents the forecast of nuclear power generating capacities that is used for the spent fuel projections. The nuclear power generating capacities are based on the April 1977 "Low Growth Case" of ERDA's Office of Planning, Analysis, and Evaluation ${ }^{1}$ through the year 2000, and on a projection by Battelle - Pacific Northwest Laboratories ${ }^{2}$ thereafter. The "Low Growth Case" is based on the premise that reprocessing is indefinitely 
deferred. The Battelle forecast assumes that the nuclear Industry consumes 3.7 million short tons of $\mathrm{U}_{3} \mathrm{O}_{8}$ over 1 ts operating lifetime with an enrichment tails assay of $0.1 \%{ }^{235} \mathrm{U}$. In addition, it assumes the following reactor capacity factors:

$\begin{array}{cc}\text { Year of operation } & \text { Capacity factor } \\ \begin{array}{c}1 \\ 1\end{array}-3 & 42 \% \\ 4-15 & 65 \% \\ 16-40 & \text { Declines } 2 \% / \text { year to } 40 \%\end{array}$

This is equivalent to a 40-year 1ifetime capacity factor of $56 \%$.

\section{REACTOR AND FUEL CHARACTERISTICS}

The pertinent physical and operational characteristics of the LWRs used in this study are given in Table 2.1. The characteristics of the reference uranium-enriched LWRs were taken from BWR and PWR standard safety analysis reports ${ }^{3,4}$ and are based on $1250 \mathrm{MW}$ of electrical generation, burnups of 27,500 and 33,000 MWdmetric ton for BWRs and PWRs, respectively, and charge-discharge rates based on an $80 \%$ reactor capacity factor.

The physical characteristics of the fresh fuel assemblies are given in Table 2.2. Additional and more-detailed characteristics can be found in ref. 5 .

The isotopic buildup and depletion in the two types of reactors were calculated with a modified version of the ORIGEN computer code, designated ORIGEN2. Approximately 200 of the cross sections used in ORIGEN2 resulted from BWR and PWR reactor physics calculations using the XSDRNPM ${ }^{6}$ and 
CITATION $^{7}$ computer codes. These calculations considered the buildup and depletion of 38 actinide isotopes between ${ }^{232} \mathrm{Th}$ and ${ }^{253} \mathrm{Es}$. Eighteen of these actinide cross sectlons vary with burnup during the ORIGEN2 irradiation calculations.

Table 2.3 presents the grams of elements charged, Tables 2.4 and 2.5 the grams of elements discharged, Tables 2.6 through 2.8 the curies of radioactivity, Tables 2.9 through 2.11 the thermal power, and Tables 2.12 through 2.14 the photon emission rates for one BWR assembly irradiated to an average burnup of $27,500 \mathrm{MWd} /$ metric ton at an average specific power of $25.9 \mathrm{MW} /$ metric ton. In addition, the total curies, the thermal power, and the neutron emission rates from spontaneous fissions and from $\alpha, n$ reactions in the fuel are given in Tables 2.15 through 2.18, respectively.

The same properties organized in identical order are presented in Tables 2.19 through 2.34 for a PWR assembly irradiated to 33,000 MWd/metric ton at $37.5 \mathrm{MW} /$ metric ton.

In these tables, a cutoff value of $0.1 \%$ of the total was used in determining which elements or nuclides are specifically identified. However, the total is representative of all elements or nuclides calculated by the computer code.

\section{PROJECTIONS OF SPENT FUEL TO BE DISCHARGED}

The nuclear generating capacity and mass flow information presented in Sects. 1 and 2 was used in the KWIKPLAN code ${ }^{8}$ to estimate the quantities of spent fuel discharged annually. These data were then used with ORIGEN2 to calculate the properties of the spent fuel accumulated through the year 2031 . 
In these projections, allowance was made for 2342 metric tons of uranium reported by ERDA to be in storage as commercial spent fuel at the end of 1976.9

Three different projections of spent fuel inventorles were made. One of these was concerned with the total inventory of spent fuel, as generated, in the United States through the year 2031. The other two were concerned with spent fuel inventories at SURFF.

The spent fuel inventory at SURFF was governed by three requirements: (1) SURFF was to begin receiving spent fuel in 1985 , (2) the inventory was to be examined with respect to accepting 5-year-old and older or 10-year-old and older spent fuel, and (3) the backlog of unshipped spent fuel was to be worked off by 1995 in both cases. These requirements were met by using specific shipping schedules for the 5-year-old case and the 10-year-old case.

The shipping schedules, Tables 3.1 and 3.2, extend only through 1995 . After this time, the amount of spent fuel shipped annually is the amount of 5- or 10-year-old spent fuel that is available for shipment each year. (However, this is not the case in the years 1985 through 1994 because of the influence of the backlog of spent fue1.)

All shipping is done on the basis of the oldest spent fuel being shipped first. In the 10-year-old spent fuel case, the shipping schedule from 1985 through 1989 is based on the amount of 10-year-old spent fuel being generated, even though the fuel being shipped is older. The shipping schedule for the second 5-year period is different because the remaining backlog is greater than the amount of 10 -year-old spent fuel being generated. To compensate for that, the remaining backlog was evenly distributed over the years 1990 through 1994. 
The shipping schedule for 5-year-old spent fuel differs from the 10-year-old case because the backlog of 5-year-old fuel is more than five times that of the 10-year-old spent fuel. Hence, the shipping schedule exceeded the amount of 5-year-old spent fuel being generated because the backlog had to be distributed over 10 years in order to meet the criterion of working off the backlog by 1995 .

Tables 3.3 through 3.24 present the accumulations of discharged fuel In the United States. The projected total accumulated properties are summarized in Table 3.3. The accumulated grams of radioisotopes are given in Tables 3.4 through 3.6, the curles in Tables 3.7 through 3.9 , the thermal decay power (includes contributions from all types of decay) of the isotopes in Tables 3.10 through 3.12, the ingestion toxicities in Tables 3.13 through 3.15, and the inhalation toxicities in Tables 3.16 through 3.18. Toxicities are expressed in the units of cubic meters of water or air necessary to dilute the isotopes to those values specified in the radiation concentration guides (10 CFR 20) as the maximum acceptable for unrestricted use. In addition, the accumulated grams and curies of radioisotopes as a function of age after 2031 are given in Tables 3.19 through 3.24 .

Accumulations at SURFF of spent fuel shipped 5 years after discharge from the reactors are presented in Tables 3.25 through 3.43 , and accumulations based on the assumption that the spent fuel is shipped 10 years after reactor discharge are given in Tables 3.44 through 3.62 .

A cutoff value of $0.01 \%$ of the total was employed for each individual isotope identified in these tables. The total, however, represents all the Isotopes included in the computer calculations. 


\section{NAC PROJECTIONS}

The Nuclear Assurance Corporation (NAC) ${ }^{10}$ has made projections of the amount of spent fuel to be discharged through 1986. These projections, which are presented in Table 4.1, reflect the utilities' expectations for additional commitments and more efficlent construction and licensing schedules in order to meet the nation's energy demand.

The NAC projections differ by up to $50 \%$ from the projections in this report. Several factors can account for this difference. First, the SURFF projections are based on the more conservative and more cautious ERDA "Low Growth Case," which forecasts lower installed capacities than NAC. Second, the SURFF projections of discharged spent fuel are based on reactor capacity factors, while the NAC projections have used individual utility discharge strategies. Finally, the SURFF projections use reactor models to project the amount of fuel discharged. These models cannot reflect the characteristics of all the presently operating reactors exactly; therefore, some deviations from the NAC projections arise from the use of such models. 


\section{REFERENCES}

1. E. J. Hanrahan, R. H. Williamson, and R. W. Bown, "World Requirements and Supply of Uranium," presented at the Atomic Industrial Forum Fuel Cycle Conference 1977, Kansas C1ty, Mo., Apr. 25, 1977.

2. R. M. Fleischman, Battelle Pacific Northwest Laboratories, personal communication (June 27, 1977).

3. Westinghouse Nuclear Energy Systems, Reference Safety Analysis Report, RESAR-3, Docket STN 50-480 (1972).

4. General Electric Standard Safety Analysis Report, BWR/6, Docket STN 50-477 (1973).

5. Alternatives for Managing Wastes from Reactors and Post-Fission Operations in the LWR Fuel Cycle, Vol. 1, Pp. 2.28-2.29, ERDA 76-43 (May 1976).

6. N. M. Greene, J. L. Lucius, L. M. Petrie, W. E. Ford III, J. E. White, and R. Q. Wright, AMPX: A Modular Code System for Generating Coupled Multigroup Neutron-Gamma Libraries from ENDF/B, ORNL/TM-3706 (March 1976).

7. T. B. Fowler and D. R. Vondy, Nuclear Reactor Core Analysis Code: CITATION, ORNL/TM-2496, Rev. 2 (July 1969).

8. R. Salmon and C. W. Kee, KWIKPLAN - A Computer Program for Projecting the Annual Requirements of Nuclear Fuel Cycle Operations, ORNL/TM-5880 (June 1977).

9. LWR Spent Fuel Disposition Capabilities, ERDA 77-25 (May 1977).

10. U.S. and Free World Discharged Nuclear Fuel Storage and Transportation Analysis, reports prepared for the U.S. Energy Research and Development Administration by Nuclear Assurance Corporation, Atlanta, Ga. (April 1977). 
TABLES 
Table 1.1. Forecast of U.S. nuclear electric power generating capacity, GW(e)

\begin{tabular}{|c|c|c|c|c|c|c|c|}
\hline \multirow{2}{*}{$\begin{array}{c}\text { Calendar } \\
\text { year }\end{array}$} & \multicolumn{3}{|c|}{ Installed capacity } & \multirow{2}{*}{$\begin{array}{c}\text { Calendar } \\
\text { year }\end{array}$} & \multicolumn{3}{|c|}{ Installed capacity } \\
\hline & BWR & PWR & Total & & BWR & PWR & Total \\
\hline $\begin{array}{r}1970 \\
71 \\
72 \\
73 \\
74 \\
75 \\
76 \\
77 \\
78 \\
79\end{array}$ & $\begin{array}{r}3.1 \\
4.4 \\
7.2 \\
7.2 \\
11.7 \\
15.2 \\
17.0 \\
17.8 \\
18.6 \\
20.4\end{array}$ & $\begin{array}{r}3.3 \\
4.7 \\
7.4 \\
12.9 \\
18.2 \\
21.8 \\
25.7 \\
33.4 \\
37.5 \\
40.2\end{array}$ & $\begin{array}{r}6.4 \\
9.1 \\
14.6 \\
20.1 \\
29.9 \\
37.0 \\
42.7 \\
51.2 \\
56.1 \\
60.6\end{array}$ & $\begin{array}{r}2000 \\
01 \\
02 \\
03 \\
04 \\
05 \\
06 \\
07 \\
08 \\
09\end{array}$ & $\begin{array}{l}121.1 \\
124.9 \\
128.6 \\
132.5 \\
136.3 \\
140.1 \\
144.0 \\
147.8 \\
151.6 \\
155.4\end{array}$ & $\begin{array}{l}258.6 \\
266.5 \\
274.3 \\
282.4 \\
290.5 \\
298.6 \\
306.7 \\
314.8 \\
321.9 \\
328.8\end{array}$ & $\begin{array}{l}379.7 \\
391.4 \\
402.9 \\
414.9 \\
426.8 \\
438.7 \\
450.7 \\
462.6 \\
473.5 \\
484.2\end{array}$ \\
\hline $\begin{array}{r}1980 \\
81 \\
82 \\
83 \\
84 \\
85 \\
86 \\
87 \\
88 \\
89\end{array}$ & $\begin{array}{l}22.0 \\
23.3 \\
25.5 \\
28.1 \\
32.7 \\
37.7 \\
42.3 \\
46.5 \\
50.7 \\
55.2\end{array}$ & $\begin{array}{r}43.8 \\
47.5 \\
56.8 \\
65.4 \\
76.5 \\
88.9 \\
98.3 \\
106.7 \\
115.1 \\
124.1\end{array}$ & $\begin{array}{r}65.8 \\
70.8 \\
82.3 \\
93.5 \\
109.2 \\
126.6 \\
140.6 \\
153.2 \\
165.8 \\
179.3\end{array}$ & $\begin{array}{r}2010 \\
11 \\
12 \\
13 \\
14 \\
15 \\
16 \\
17 \\
18 \\
19\end{array}$ & $\begin{array}{l}157.8 \\
160.3 \\
161.3 \\
165.2 \\
164.5 \\
165.3 \\
166.9 \\
169.9 \\
174.2 \\
176.2\end{array}$ & $\begin{array}{l}335.9 \\
342.6 \\
348.0 \\
350.6 \\
353.3 \\
356.5 \\
361.2 \\
361.6 \\
364.4 \\
369.8\end{array}$ & $\begin{array}{l}493.7 \\
502.9 \\
509.3 \\
515.8 \\
517.8 \\
521.8 \\
528.1 \\
531.5 \\
538.6 \\
546.0\end{array}$ \\
\hline \multirow[t]{2}{*}{$\begin{array}{r}1990 \\
91 \\
92 \\
93 \\
94 \\
95 \\
96 \\
97 \\
98 \\
99\end{array}$} & $\begin{array}{r}60.2 \\
65.4 \\
70.9 \\
76.4 \\
82.6 \\
89.1 \\
95.6 \\
102.1 \\
108.7 \\
115.2\end{array}$ & $\begin{array}{l}134.2 \\
144.8 \\
156.1 \\
167.4 \\
180.1 \\
193.4 \\
206.7 \\
220.0 \\
233.2 \\
246.5\end{array}$ & $\begin{array}{l}194.4 \\
210.2 \\
227.0 \\
243.8 \\
262.7 \\
282.5 \\
302.3 \\
322.1 \\
341.9 \\
361.7\end{array}$ & $\begin{array}{r}2020 \\
21 \\
22 \\
23 \\
24 \\
25 \\
26 \\
27 \\
28 \\
29 \\
30\end{array}$ & $\begin{array}{l}178.5 \\
181.0 \\
182.6 \\
183.9 \\
183.1 \\
181.9 \\
181.1 \\
182.1 \\
181.7 \\
181.0 \\
179.8\end{array}$ & $\begin{array}{l}374.3 \\
378.7 \\
377.5 \\
377.0 \\
373.9 \\
369.6 \\
368.3 \\
366.9 \\
366.6 \\
365.6 \\
363.6\end{array}$ & $\begin{array}{l}552.8 \\
559.7 \\
560.1 \\
560.9 \\
557.0 \\
551.5 \\
549.4 \\
549.0 \\
548.3 \\
546.6 \\
543.4\end{array}$ \\
\hline & & & & 31 & 179.8 & 360.0 & 539.8 \\
\hline
\end{tabular}


Table 2.1. Assumed characteristics of 11ght water reactors

\begin{tabular}{|c|c|c|}
\hline & BWR & PWR \\
\hline Electric power, MW(e) & 1,250 & 1,250 \\
\hline Thermal power, $M W(t)$ & 3,800 & 3,800 \\
\hline $\begin{array}{l}\text { Average specific power, } \\
M W(t) / \text { metric ton }\end{array}$ & 25.9 & 37.5 \\
\hline $\begin{array}{l}\text { Average fuel burnup, } \\
\text { MWd/metric ton }\end{array}$ & 27,500 & 33,000 \\
\hline $\begin{array}{l}\text { Fuel residence time at } 80 \% \\
\text { capacity factor, days }\end{array}$ & 1,328 & 1,100 \\
\hline $\begin{array}{l}\text { Steady-state charge, } \mathrm{kg} / \text { yea } \\
235 \mathrm{U} \\
\text { Total U }\end{array}$ & $\begin{array}{r}1,110 \\
40,368\end{array}$ & $\begin{array}{r}1,081 \\
33,778\end{array}$ \\
\hline $\begin{array}{l}\text { Steady-state discharge, kg } \\
{ }_{235}{ }^{\mathrm{U}} \\
\text { Total } \mathrm{U} \\
\text { Fissile } \mathrm{Pu}^{\mathrm{b}} \\
\text { Total Puc } \\
\text { Total actinides }\end{array}$ & $\begin{array}{r}304 \\
38,871 \\
237 \\
340 \\
39,223\end{array}$ & $\begin{array}{r}268 \\
32,299 \\
208 \\
307 \\
32,629\end{array}$ \\
\hline
\end{tabular}

\footnotetext{
a Based on full power and fuel charged. $\mathrm{b}_{239} \mathrm{Pu}+{ }^{241} \mathrm{Pu}$.$$
\mathrm{C}_{238} \mathrm{Pu}+{ }^{239} \mathrm{Pu}+{ }^{240} \mathrm{Pu}+{ }^{241} \mathrm{Pu}+{ }^{242} \mathrm{Pu} \text {. }
$$ 
Table 2.2. Physical characteristics of LWR fuel assemblies

\begin{tabular}{|c|c|c|}
\hline & BWR & PWR \\
\hline Overall assembly length, m & 4.470 & 4.059 \\
\hline Cross section, $\mathrm{cm}$ & $13.9 \times 13.9$ & $21.4 \times 21.4$ \\
\hline Fuel element length, m & 4.064 & 3.851 \\
\hline Active fuel height, $\mathrm{m}$ & 3.759 & 3.658 \\
\hline Fuel element OD, $\mathrm{cm}$ & 1.252 & 1.07 \\
\hline Fuel element array & $8 \times 8$ & $15 \times 15$ \\
\hline Assembly total weight, $\mathrm{kg}$ & 275.7 & 657.9 \\
\hline Uranium/assembly, $\mathrm{kg}$ & 183.3 & 461.4 \\
\hline $\mathrm{UO}_{2}$ /assembly, $\mathrm{kg}$ & 208.0 & 523.4 \\
\hline Zircaloy/assembly, kg & $57.9^{\mathrm{a}}$ & $108.4^{b}$ \\
\hline Hardware/assembly, kg & $9.77^{c}$ & $26.1^{d}$ \\
\hline Total metal/assembly, kg & 67.7 & 134.5 \\
\hline Nominal volume/assembly, $\mathrm{m}^{3}$ & $0.0864^{e}$ & $0.186^{e}$ \\
\hline
\end{tabular}

ancludes Zircaloy fuel-element spacers.

${ }^{b}$ Includes Zircaloy control-rod guide thimbles.

cIncludes stainless steel tie-plates and Inconel springs.

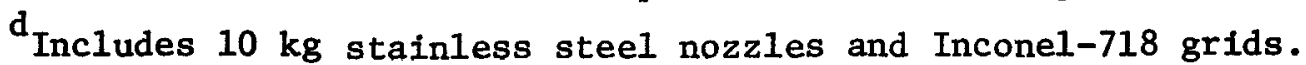

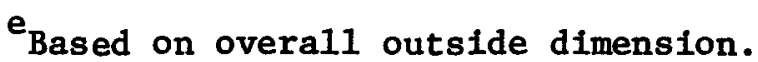


Table 2.3. Total grams charged to a BWR fuel assembly

\begin{tabular}{cc}
$\begin{array}{c}\text { Elements or } \\
\text { isotopes }\end{array}$ & $\begin{array}{c}\text { Grams } \\
\text { charged }\end{array}$ \\
\hline $\mathrm{N}$ & $2.555 \times 10^{1}$ \\
$\mathrm{O}$ & $2.474 \times 10^{4}$ \\
$\mathrm{Si}$ & $9.491 \times 10^{1}$ \\
$\mathrm{Cr}$ & $1.986 \times 10^{3}$ \\
$\mathrm{Mn}$ & $1.865 \times 10^{2}$ \\
$\mathrm{Fe}$ & $6.644 \times 10^{3}$ \\
$\mathrm{Ni}$ & $1.157 \times 10^{3}$ \\
$\mathrm{Zr}$ & $1.012 \times 10^{5}$ \\
$\mathrm{Sn}$ & $1.654 \times 10^{3}$ \\
$\mathrm{Gd}$ & $1.380 \times 10^{5}$ \\
$235 \mathrm{U}$ & $1.782 \times 10^{5}$ \\
$238 \mathrm{U}$ & $5.041 \times 10^{3}$ \\
\hline
\end{tabular}

ampurities of $25 \mathrm{ppm}$ of nitrogen and $1 \mathrm{ppm}$ of lithium, in addition to others, are found in the fuel. 
Table 2.4. Grams of fission-product elements in a BWR assembly

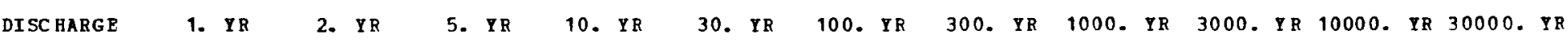
$\begin{array}{llllllllllllll}8.590 \mathrm{E}-03 & 8.120 \mathrm{E}-03 & 7.675 \mathrm{E}-03 & 6.481 \mathrm{E}-03 & 4.890 \mathrm{E}-03 & 1.584 \mathrm{E}-03 & 3.065 \mathrm{E}-05 & 3.906 \mathrm{E}-10 & 2.883 \mathrm{E}-27 & 0.0 & 0.0 \\ 3.052 \mathrm{E}-05 & 3.052 \mathrm{E}-05 & 3.052 \mathrm{E}-05 & 3.052 \mathrm{E}-05 & 3.052 \mathrm{E}-05 & 3.052 \mathrm{E}-05 & 3.052 \mathrm{E}-05 & 3.052 \mathrm{E}-05 & 3.052 \mathrm{E}-05 & 3.052 \mathrm{E}-05 & 3.052 \mathrm{E}-05 & 0.0 & 3.052 \mathrm{E}-05\end{array}$ 2.281E-05 2.28 1E-05 2.281E-05 2.281E-05 2.281E-05 2.281E-05 2.281E-05 2.281E-05 2.281E-05 2.280 E-05 2.276E-05 2.265E-05

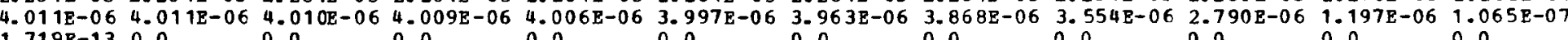

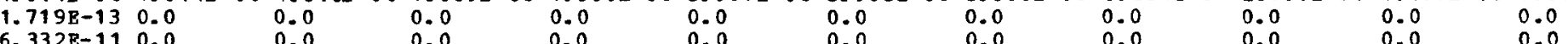

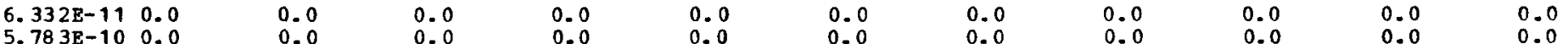

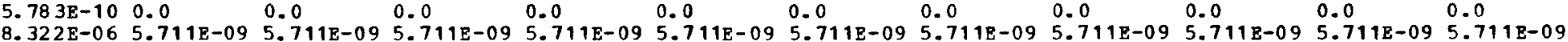

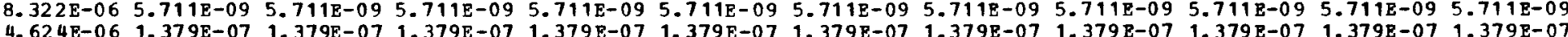
4. $624 \mathrm{E}-06$ 1.379E-07 1.379E-07 1.379E-07 1.379E-07 1.379E-07 1.3798-07 1.379E-07 1.379E-07 1.379E-07 1.379E-07 $1.379 \mathrm{E}-07$

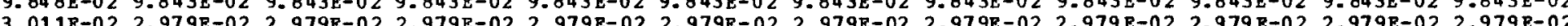

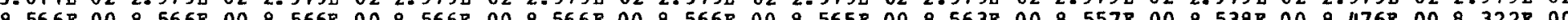

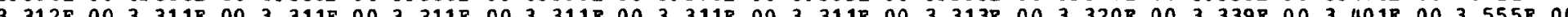
5.602 015.5708015 .5572015 .5018015 .4298015 .2928015 .2418015 .2408015 .2408015 .2408015 .2408015 .240801

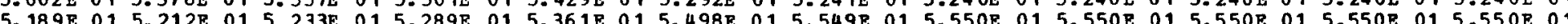

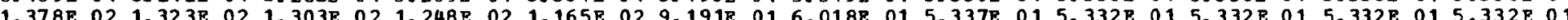

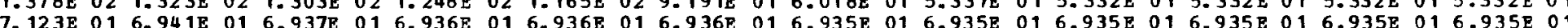

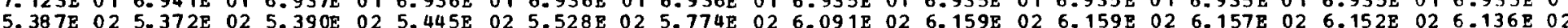

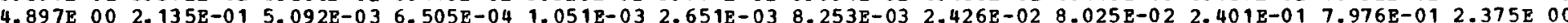

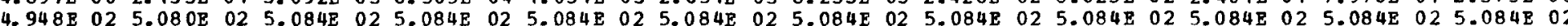

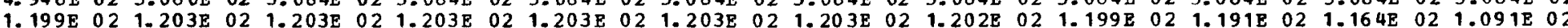
$\begin{array}{lllllllllllllllllllllllll}3.604 \mathrm{E} & 02 & 3.429 \mathrm{E} & 02 & 3.370 \mathrm{E} & 02 & 3.318 \mathrm{E} & 02 & 3.311 \mathrm{E} & 02 & 3.311 \mathrm{E} & 02 & 3.311 \mathrm{E} & 02 & 3.312 \mathrm{E} & 02 & 3.315 \mathrm{E} & 02 & 3.322 \mathrm{E} & 02 & 3.349 \mathrm{E} & 02 & 3.423 \mathrm{E} & 02\end{array}$ $6.855 \mathrm{E} 017.429 \mathrm{E} 017.430 \mathrm{E} \quad 017.430 \mathrm{E} \quad 017.430 \mathrm{E} \quad 017.430 \mathrm{E} \quad 017.430 \mathrm{E} \quad 01 \quad 7.430 \mathrm{E} \quad 017.430 \mathrm{E} \quad 017.430 \mathrm{E} \quad 017.430 \mathrm{E} \quad 017.430 \mathrm{E} \quad 01$ $\begin{array}{llllllllllllllllllllllllllll}1.851 \mathrm{E} & 02 & 1.969 \mathrm{E} & 02 & 2.027 \mathrm{E} & 02 & 2.079 \mathrm{E} & 02 & 2.086 \mathrm{E} & 02 & 2.086 \mathrm{E} & 02 & 2.086 \mathrm{E} & 02 & 2.086 \mathrm{E} & 02 & 2.086 \mathrm{E} & 02 & 2.086 \mathrm{E} & 02 & 2.086 \mathrm{E} & 02 & 2.085 \mathrm{E} & 02\end{array}$

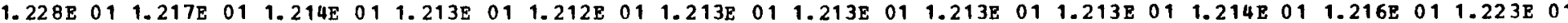

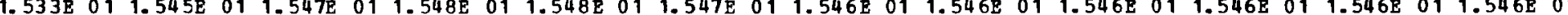
3.61 1E-01 3.785E-01 3.802E-01 3.846E-01 3.907E-01 4.046E-01 4. 131E-01 4. 134E-01 4. 134E-01 4. 134E-01 4.134E-01 4.134E-0 $1.311 \mathrm{E} 011.298 \mathrm{E} 01$ 1.296E 011 1.296E 01 1.296E 01 1.296E 01 1.295E 01 1.295E 01 1.293E 01 1.287E 01 1.268E 01 1.219E 01

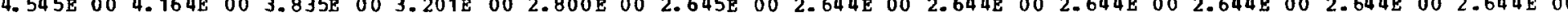

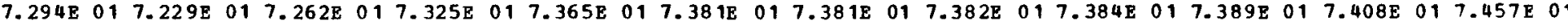
$\begin{array}{llllllllllllllllllllllll}3.657 \mathrm{E} & 01 & 3.592 \mathrm{E} & 01 & 3.594 \mathrm{E} & 01 & 3.594 \mathrm{E} & 01 & 3.594 \mathrm{E} & 01 & 3.594 \mathrm{E} & 01 & 3.594 \mathrm{E} & 01 & 3.594 \mathrm{E} & 01 & 3.594 \mathrm{E} & 01 & 3.594 \mathrm{E} & 01 & 3.593 \mathrm{E} & 01 & 3.591 \mathrm{E} & 01\end{array}$ 8. 015E 02 8.017E 02 8.017E 02 8.017E 02 8.017 E 02 8.017E 02 8.017E 02 8.017E 02 8.017E 02 8.017E 02 8.017E 02 8.017E 02

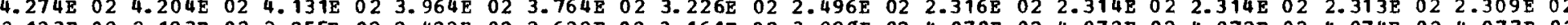

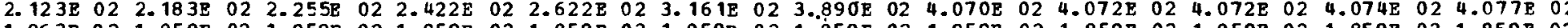

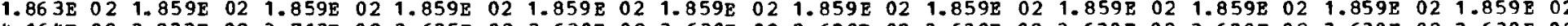

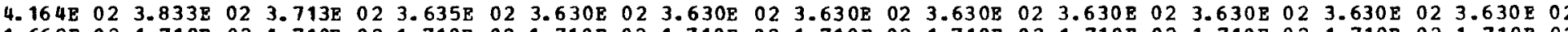

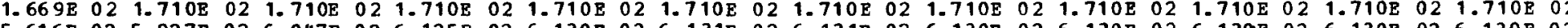

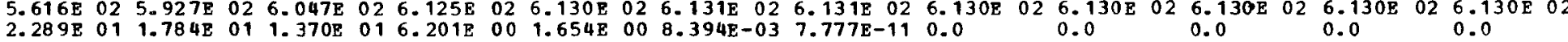

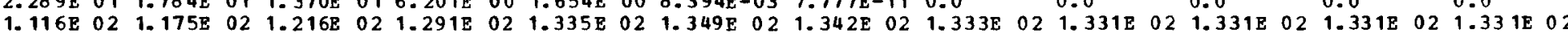
2.396E 01 2. 303E 01 2.241E 01 2.092E 01 1.926E 01 1.712E 01 1.734E 01 1.817E 01 1.842E 01 1.842E 01 1.842E 01 1.842E 01

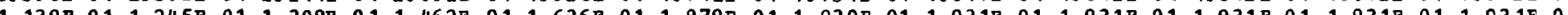

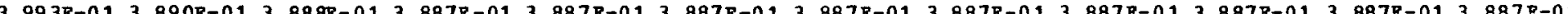
1.93

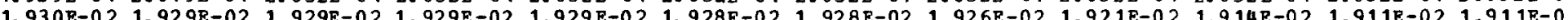

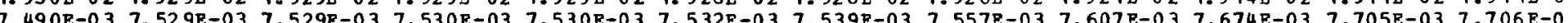

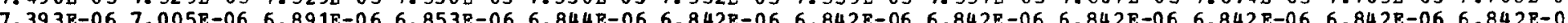

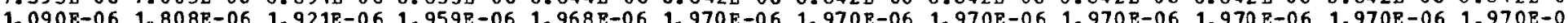

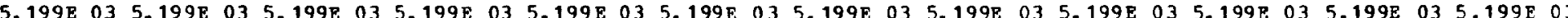


Table 2.5. Grams of heavy elements in a BWR assembly

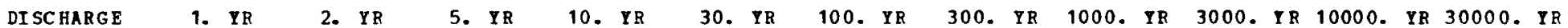

4. $414 \mathrm{E}-02 \quad 7.391 \mathrm{E}-02 \quad 8.460 \mathrm{E}-02 \quad 1.034 \mathrm{E}-01 \quad 1.351 \mathrm{E}-01 \quad 2.802 \mathrm{E}-01 \quad 8.082 \mathrm{E}-01 \quad 1.974 \mathrm{E} \quad 00 \quad 4.227 \mathrm{E} \quad 00 \quad 6.835 \mathrm{E} \quad 00 \quad 1.188 \mathrm{E} \quad 01 \quad 1.904 \mathrm{E} \quad 01$

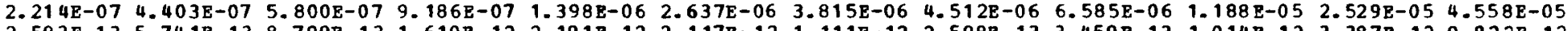

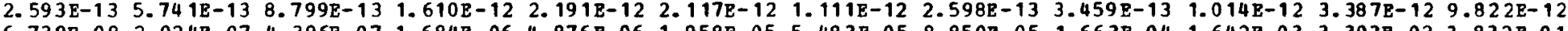

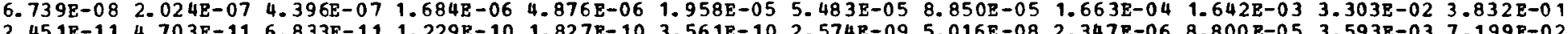

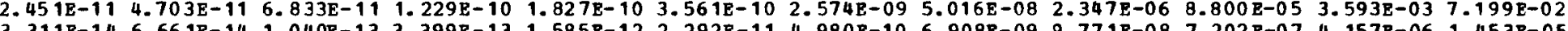

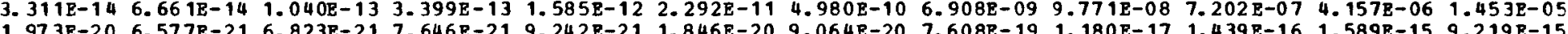

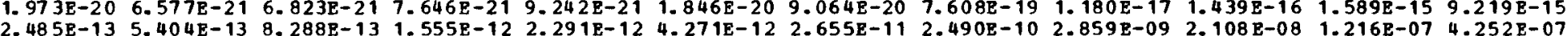

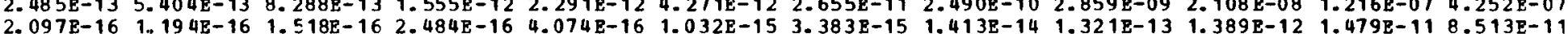
4.338E-09 8.091E-09 1.248E-08 2.880E-08 6.665E-08 3.858E-07 3.992E-06 3.875E-05 $4.453 \mathrm{E}-04 \quad 3.282 \mathrm{E}-03 \quad 1.895 \mathrm{E}-02 \quad 6.623 \mathrm{E}-02$ $1.083 \mathrm{E}-092.221 \mathrm{E}-09$ 3. $352 \mathrm{E}-096.695 \mathrm{E}-09$ 1.212E-08 3.239E-08 9.582E-08 2.688E-07 8.992E-07 $2.617 \mathrm{E}-06 \quad 8.518 \mathrm{E}-06 \quad 2.378 \mathrm{E}-05$ $2.482 \mathrm{E}-04$ 3. $453 \mathrm{E}-04 \quad 4.430 \mathrm{E}-04 \quad 7.384 \mathrm{E}-04 \quad 1.238 \mathrm{E}-03$ 3. $322 \mathrm{E}-03$ 1.151E-02 3.883E-02 1.423E-01 4.392E-01 1.465E $00 \quad 4.162 \mathrm{E}$ 00 5. 823E-05 5. $913 \mathrm{E}-05$ 6. $106 \mathrm{E}-05$ 6. $603 \mathrm{E}-05$ 7.167E-05 $9.825 \mathrm{E}-05$ 1.915E-04 4.574E-04 $1.384 \mathrm{E}-03$ 4.009E-03 $1.298 \mathrm{E}-02 \quad 3.602 \mathrm{E}-02$ 1.765E 05 1.765E 05 1.765E 05 1.765E 05 1.765E 05 1.765E 05 1.765E 05 1.765E 05 1.765E 05 1.767E 05 1.769E 05 1.773E 05

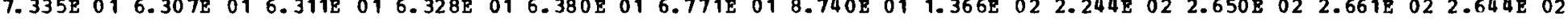

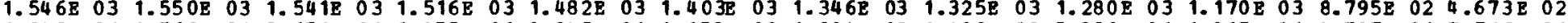

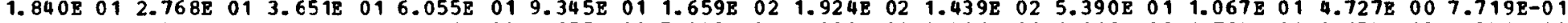

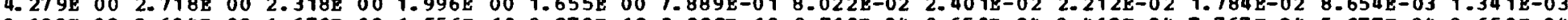
8.122E-09 3.684E-09 1.670E-09 1.556E-10 2.978E-12 3.99gE-19 8.719E-24 8.650E-24 8.4128-24 7.767E-24 5.8778-24 $2.650 \mathrm{E}-24$

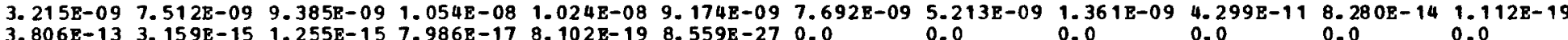

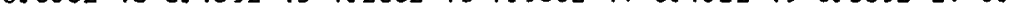

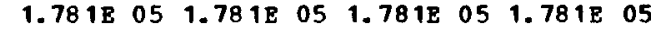


Table 2.6. Curies of light-element isotopes in a BWR assembly
DISC HARGE
1. $Y R$
2. $Y R$
5. YR
10. YR
30. YR
100. IR
300. Y
1000. IR 3000 . IR 10000. YR 30000. TR

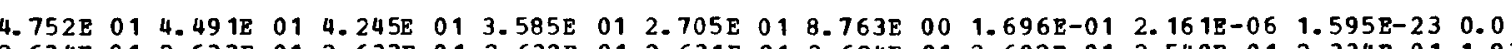

0.0 $2.634 \mathrm{E}-012.633 \mathrm{E}-012.633 \mathrm{E}-012.632 \mathrm{E}-012.631 \mathrm{E}-012.624 \mathrm{C}$ $100.0-0.00$ .

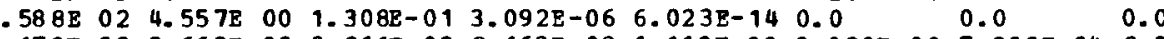
$\begin{array}{lll}0.0 & 0.0 \\ 0 & 0.0 & 0\end{array}$

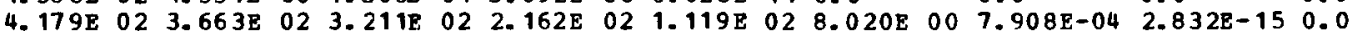
(1.9248-03 $1.990 \mathrm{~B}-03$

.508E-01 1.508E-01 1.508E-01 1.508E-01 1.508E-01 1.507E-01 1.506E-01 1.504E-01

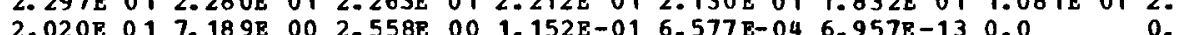
(1)

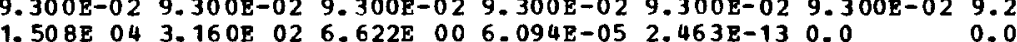
$00298-029.29$ .3968001 .22

7.032E-03 1.160E-02 1.591E-02 2.744E-02 4.273E-02 7.399E-02 8.809E-02

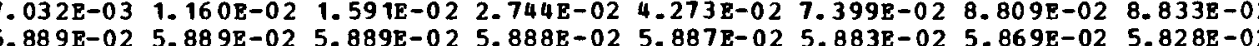

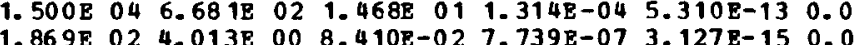
0.0 0.0 1. $434 \mathrm{E}-03$ 1. $433 \mathrm{E}-03$ 1.433E-03 $1.432 \mathrm{E}-03$ 1.430E-03 $1.424 \mathrm{E}-03 \quad 1.401 \mathrm{E}-03$ 1.338E-03 $\begin{array}{llllll} & \end{array}$ 2.8948 02 3.202E 01 3.542E $004.916 \mathrm{E}-03$ 8.150E-08 $6.155 \mathrm{E}-270.0$ 2.224E 03 7. $915 \mathrm{E} 02$ 2.817 $021.269 \mathrm{E}$ 01 $7.243 \mathrm{E}-027.676 \mathrm{E}-110.0$ 0.0 1. (.482E 01 2.084E 00 5.792E-03 3.183E-07 2.903E-24 0.0 $3.789 \mathrm{E}-03$ $1.369 \mathrm{E} 021.207 \mathrm{E} \quad 02$ 9.385E 01 4.382E $011.231 \mathrm{E}$ 01 $7.685 \mathrm{E}-021.468 \mathrm{E}-090.0$

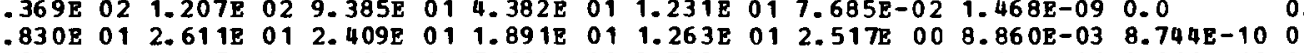

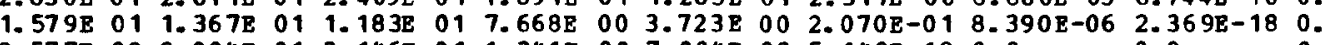

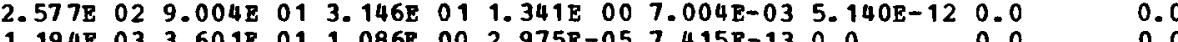
0.0

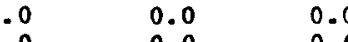
$\begin{array}{ll}0.0 \\ 0.0 & 0.0\end{array}$ $0.0 \quad 0.0$ $\begin{array}{lll}0.0 & 0.0 \\ 369 \mathrm{~B}-01 & 1.383 \mathrm{E}-01 & 1.163 \mathrm{E}-01\end{array}$ $\begin{array}{lll}3.505 \mathrm{E}-09 & 0.0 & 0.0 \\ 0.0 & 0.0\end{array}$ $227 \mathrm{E}-023.505 \mathrm{E}-090.00000$ $\begin{array}{llll}9.0 & 0.0 & 0.0 & 0.0 \\ 0.293 \mathrm{E}-02 & 9.280 \mathrm{E}-02 & 9.232 \mathrm{E}-02 & 9.099 \mathrm{E}-02\end{array}$ $\begin{array}{llll}0.0 & 0.0 & 0.0 & 0.0\end{array}$ 0

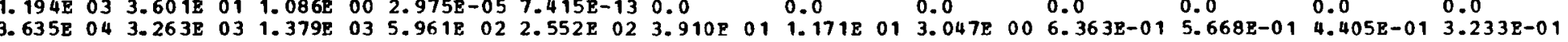

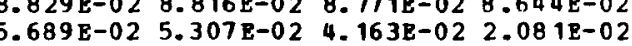
$\begin{array}{llll}0.0 & 0.0 & 0.0 & 0.0\end{array}$

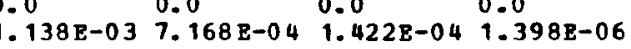
$\begin{array}{cccc}0.0 & 0.0 & 0.0 & 0.0\end{array}$ $\begin{array}{llll}0.0 & 0.0 & 0.0 & 0.0 \\ 0.0 & 0.0 & 0.0 & 0.0\end{array}$ $\begin{array}{llll}0.0 & 0.0 & 0.0 & 0.0 \\ 2.300 \mathrm{~B}-07 & 2.060 \mathrm{~B}-19 & 0.0 & 0.0\end{array}$ $\begin{array}{llll}0.0 & 0.0 & 0.0 & 0.0\end{array}$ $\begin{array}{llll}0.0 & 0.0 & 0.0 & 0.0 \\ 0 & 0.0 & 0.0 & 0.0\end{array}$ 0.0 $\begin{array}{ll}0 & 0.0 \\ 0 & 0.0\end{array}$ 0.0 0.0 0.0 0.0 0.0
0.0
0.0

TOTAL 
Table 2.7. Curies of fission-product isotopes in a BWR assembly

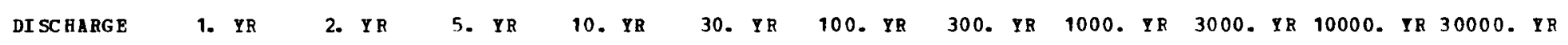

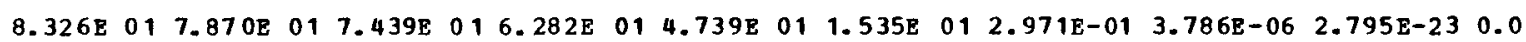

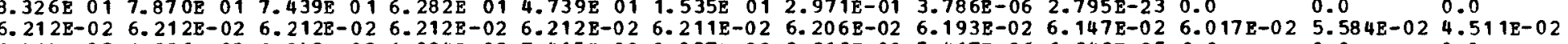

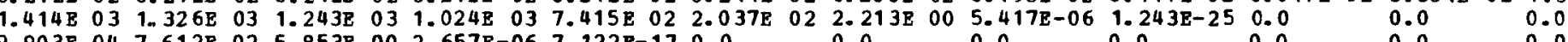

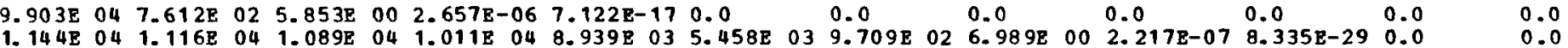

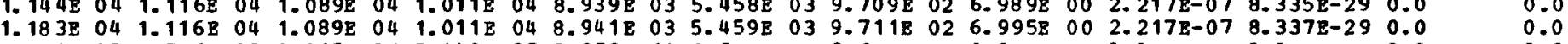

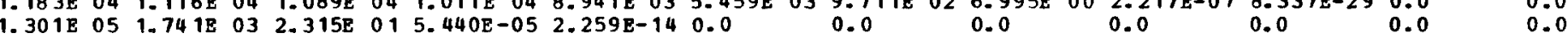
$\begin{array}{lllll}4 & \end{array}$

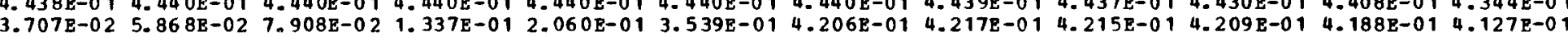

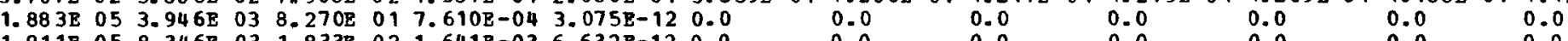

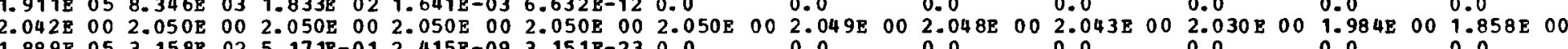

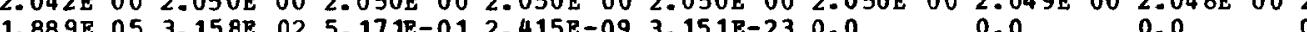

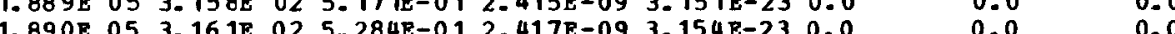

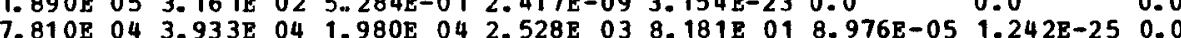
$8.383 \mathrm{E} 043.933 \mathrm{E} 04 \mathrm{~T}$ 1.980E $042.528 \mathrm{E} 038.181 \mathrm{E} 018.976 \mathrm{E}-05$ 1.242E-25 0.0 (.7398-02 $1.7398-021.7398-021.739 E-021.7398-021.7398-021.739 E-021.7398-021$. $2.064 \mathrm{E} 03$ 1.6138 $031.2518035 .842 \mathrm{E} 021.641 \mathrm{E} 021.0248001 .956 \mathrm{E}-080.0$

RD 103

R日1034

SB 125

TE 125 M SB126 SB126: SB 126 . CS134 1.158E-01 1.158E-01 1.158E-01 1.158E-01 1.158E-01 1.157E-01 1.157E-01 1.155E-01 1.150E-01 1.134E-01 1.080E-01 9.402E-02

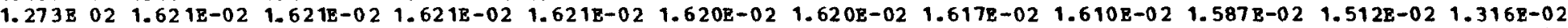
$6.310 \mathrm{E} 01$ 1.158E-01 1.158E-01 1.158E-01 1.158E-01 1.157E-01 1.157E-01 1.155E-01 1.1508-01 1.134E-01 1.080E-01 9.402E-02 $4.742 \mathrm{E}-03 \quad 4.800 \mathrm{E}-03 \quad 4.800 \mathrm{E}-03 \quad 4.800 \mathrm{E}-03 \quad 4.800 \mathrm{E}-03 \quad 4.800 \mathrm{E}-03$ 4.800E-03 4.800E-03 4.800 E-03 4.800E-03 4.798E-03 $4.794 \mathrm{E}-03$ $\begin{array}{lllllllllllllll}2.035 \mathrm{E} & 04 & 1.454 \mathrm{E} & 04 & 1.039 \mathrm{E} & 04 & 3.785 \mathrm{E} & 03 & 7.037 \mathrm{E} & 02 & 8.771 \mathrm{E}-01 & 5.177 \mathrm{E}-11 & 0.0 & 0.0 & 0.0\end{array}$

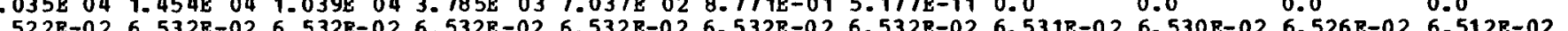

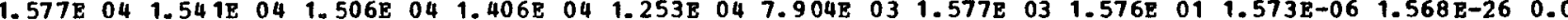

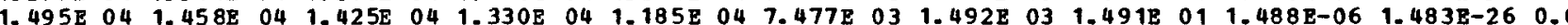

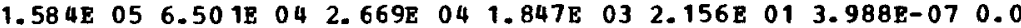
0.0 (1) 0.0 $1.592 \mathrm{E} 05 \quad 6.502 \mathrm{E} 04 \quad 2.669 \mathrm{E} 04 \mathrm{1.847E} 03 \quad 2.156 \mathrm{~B}$ 01 $3.988 \mathrm{E}-070.0$ $1.902 \mathrm{E} 03 \quad 7.802 \mathrm{E} 02 \mathrm{3} 3.203 \mathrm{E} 02 \quad 2.216 \mathrm{E} 012.587 \mathrm{E}-01$ 4.786E-09 0.0 0.0

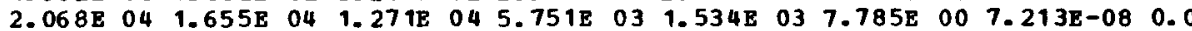
$\begin{array}{llllll} & \end{array}$

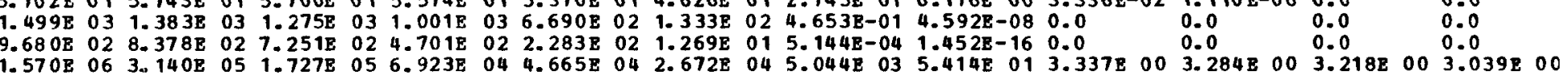
$\begin{array}{lll}0.0 & 0.0 \\ 0.0 & 0.0 & 0\end{array}$ 0.0
0.0
0.0
0.0 $\begin{array}{llll}0.0 & 0.0 & 0.0 & 0.0 \\ 0.0 & 0.0 & 0.0 & 0.0\end{array}$ 
Table 2.8. Curies of heavy-element isotopes in a BWR assembly
DI SCHARGE
1. $Y R$
2. IR 5. $Y R$
10. YR
30. $Y R$ 100. $Y R$
300. YR
000. YR 3000. YR 10000. YR 30000. YR

$2.417 \mathrm{E}-10 \quad 3.759 \mathrm{E}-10 \quad 5.737 \mathrm{E}-10 \quad 1.816 \mathrm{E}-09 \quad 7.120 \mathrm{E}-09 \quad 1.033 \mathrm{E}-07 \quad 2.245 \mathrm{E}-06 \quad 3.114 \mathrm{E}-05 \quad 4-404 \mathrm{E}-04 \quad 3.247 \mathrm{E}-03 \quad 1.874 \mathrm{E}-02 \quad 6.550 \mathrm{E}-02$ 2.995E-09 5.132E-09 7.942E-09 2.043E-08 5.497E-08 3.710E-07 3.943E-06 3.833E-05 4.404E-04 3.247E-03 1.874E-02 6.550E-02

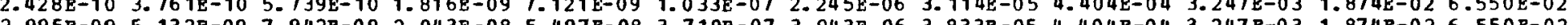
$1.995 \mathrm{E}-095.132 \mathrm{E}$

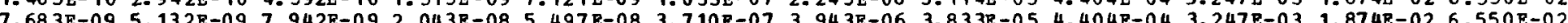
. 2.95 (2.

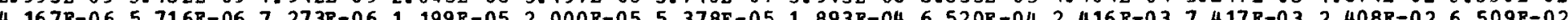

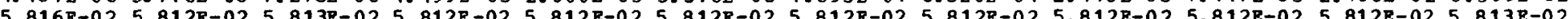

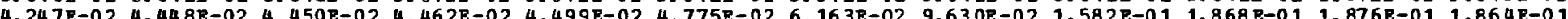
$5.861 \mathrm{E}-025,812 \mathrm{E}-025.813 \mathrm{E}-02$ 5.812E-02 5.812E-02 5.

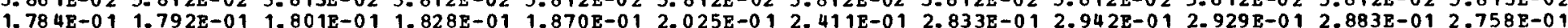
3.85 2E-02 3.85 2E-02 3.852E-02 3.853E-02 3.854E-02 3.859E-02 3.876E-02 3.925E-02 4.0878-02 4. $490 \mathrm{E}-02$ 5.398E-02 $6.149 \mathrm{E}-02$ 5.812E-02 $5.812 \mathrm{E}-02$ 5.812E-02 $5.812 \mathrm{E}-02$ 5.812R-02 5.812E-02 $5.812 \mathrm{E}-02$ 5.812E-02 $5.812 \mathrm{E}-02$ 5.812E-02 5.812E-02 $5.813 \mathrm{E}-02$

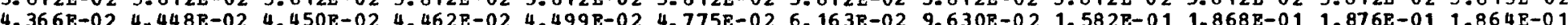

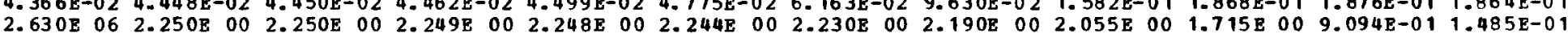
2. $885 \mathrm{E} \quad 02$ 3.130E 02 3.158E 02 3.099E 02 2.981E 02 2.552E 02 1.481E 02 3.140E $01 \quad 1.501 \mathrm{E}-01 \quad 1.907 \mathrm{E}-06 \quad 2.581 \mathrm{E}-200.0$

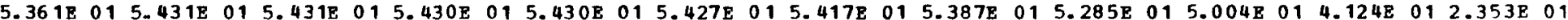

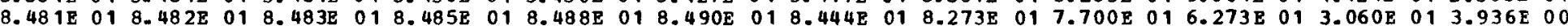
$2.039 \mathrm{E} \quad 04 \quad 1.945 \mathrm{E} \quad 04 \quad 1.854 \mathrm{E} \quad 04 \quad 1.608 \mathrm{E} \quad 04 \quad 1.268 \mathrm{E} \quad 04 \quad 4.908 \mathrm{E} 03 \quad 1.768 \mathrm{E} \quad 02 \quad 1.582 \mathrm{E}-02 \quad 2.375 \mathrm{E}-03 \quad 2.009 \mathrm{E}-03 \quad 1.117 \mathrm{E}-03 \quad 2.087 \mathrm{E}-04$

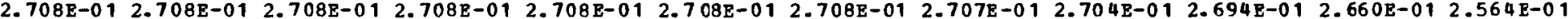

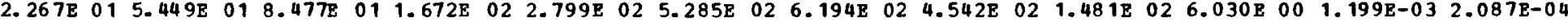

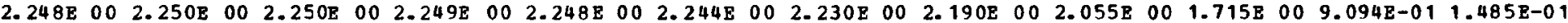

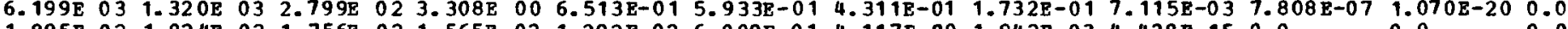

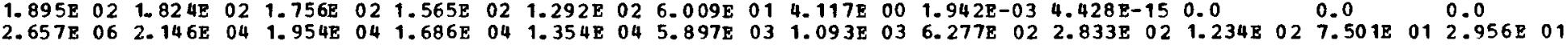


Table 2.9. Watts of light-element isotopes in a BWR assembly
DI SC HARGE
1. $\mathrm{YR}$
2. $Y R$
5. $\mathrm{YR}$
10. Y $\mathrm{P}$
30. $\mathrm{YR}$
100. Y
300. I
1000.
3000. IR 10000. YR 30000. YR

$\begin{array}{llllllllllllllll}1.690 \mathrm{E}-03 & 1.597 \mathrm{E}-03 & 1.510 \mathrm{E}-03 & 1.275 \mathrm{E}-03 & 9.620 \mathrm{E}-04 & 3.117 \mathrm{E}-04 & 6.032 \mathrm{E}-06 & 7.685 \mathrm{E}-11 & 5.673 \mathrm{E}-28 & 0.0\end{array}$

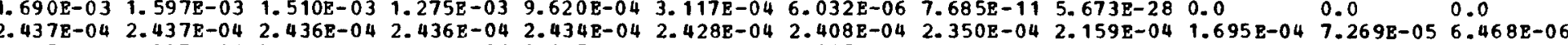
$\begin{array}{lll} & \end{array}$ $\begin{array}{lll}0.02 & 0.0 & 0.0\end{array}$

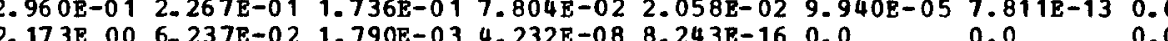
000

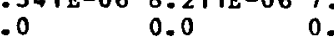

AN 54

FE 55

CO 60

CO 60

NI 63

NI 63

ZN 65

ZR 93

NB $93 \mathrm{M}$

NB 931

NB 94

HO 93

A G 108 H

I $N 113 \mathrm{H}$

SH113

SN119M

SN121M

SN123

SB125

TE125M

EO15

ED15

TB160

TA182

SOHTOT

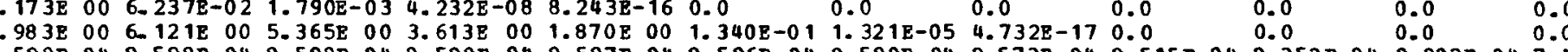

$0.0 \quad 0.0 \quad 0.0$

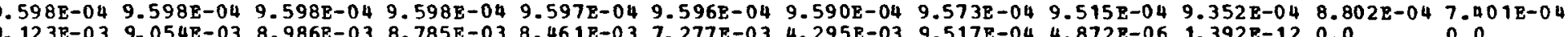

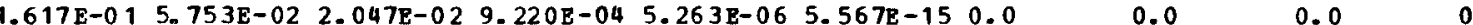

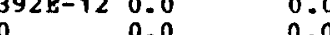

103E-05 1.103E-05 1. 103E-05 1. 103E-05 1.103E-05 1.103E-05 1.102E-05 1.102E-05 1.102E-05 1.100 E-05 1.095E-05 1.079 -05

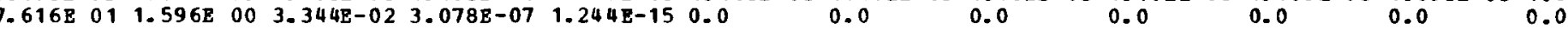

$1.250 \mathrm{E}-06 \quad 2.063 \mathrm{E}-06 \quad 2.829 \mathrm{E}-06 \quad 4.880 \mathrm{E}-06 \quad 7.599 \mathrm{E}-06 \quad 1.316 \mathrm{E}-05 \quad 1.567 \mathrm{E}-05$ 1.571E-05 $1.570 \mathrm{E}-05 \quad 1.568 \mathrm{E}-05 \quad 1.560 \mathrm{E}-05 \quad 1.537 \mathrm{E}-05$ 113E-04 6.113E-04 6.112B-04 6.112E-04 6.111E-04 6.106E-04 6.092E-04 6.050E-04 5.904E-04 5.509 B-04 4-321E-04 2-1608-04

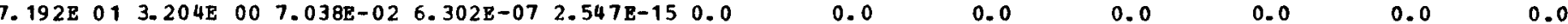

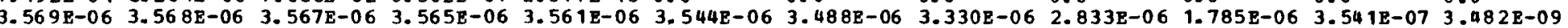
$1.149 \mathrm{E}-05$ 1. $143 \mathrm{E}-05$ 1.137E-05 $1.119 \mathrm{E}-05$ 1.089E-05 $9.791 \mathrm{E}-06 \quad 6.739 \mathrm{E}-06 \quad 2.319 \mathrm{E}-06 \quad 5.537 \mathrm{E}-08 \quad 1.286 \mathrm{E}-12 \quad 7.764 \mathrm{E}-29 \quad 0.0$

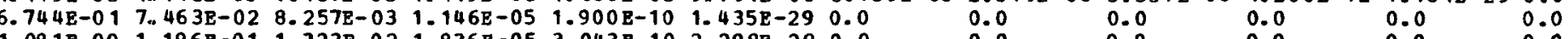
1. $081 \mathrm{E} 00$ 1. $196 \mathrm{E}-01$ 1.323E-02 $1.836 \mathrm{E}-053.043 \mathrm{E}-102.298 \mathrm{E}-290.0$

$1.173 \mathrm{E} 00 \quad 4.176 \mathrm{E}-01 \quad 1.486 \mathrm{E}-01 \quad 6.695 \mathrm{E}-03 \quad 3.821 \mathrm{E}-05 \quad 4.050 \mathrm{E}-14 \quad 0.0$

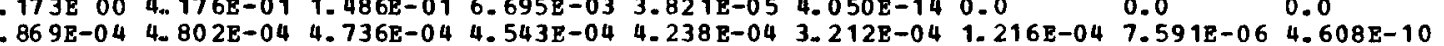

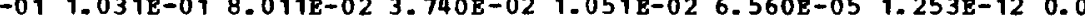
$0.0 \quad 0.0$ 0.0 $7598-120.0$

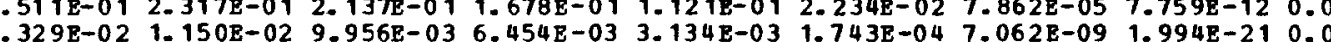
. 0.0 0.0
0.0
0.0 $\begin{array}{lll}0.0 & 0.0 \\ 0.0 & 0.0 & 0\end{array}$ $0.0 \quad 0.0$

0.0

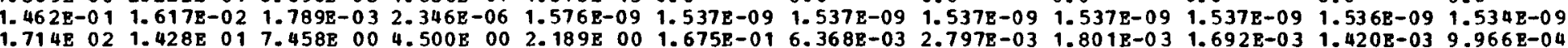
TOTAL

\footnotetext{
$\begin{array}{lllllllllllllllll}6.080 \mathrm{E} & 02 & 1.430 \mathrm{E} & 01 & 7.459 \mathrm{E} & 00 & 4.500 \mathrm{E} & 00 & 2.189 \mathrm{E} & 00 & 1.675 \mathrm{E}-01 & 6.370 \mathrm{E}-03 & 2.799 \mathrm{E}-03 & 1.802 \mathrm{E}-03 & 1.693 \mathrm{E}-03 & 1.420 \mathrm{E}-03 & 9.968 \mathrm{E}-04\end{array}$
} 
$.545 \mathrm{E}-05$
$.319 \mathrm{E}-03$

$542 \mathrm{E}-05 \quad 1.530 \mathrm{E}-05 \quad 1.498 \mathrm{E}-05$

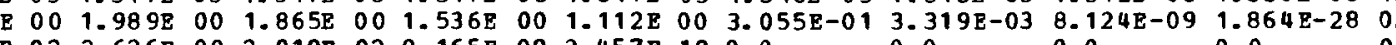

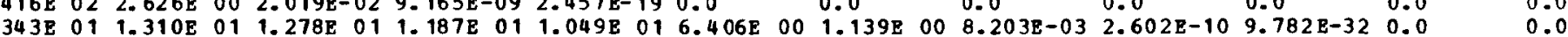

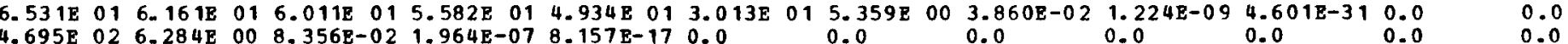

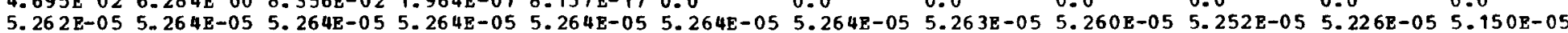

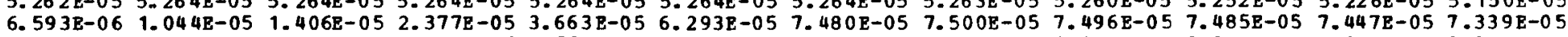
9. $509 \mathrm{E} 021.993801 \quad 4.176 \mathrm{~B}-013.843 \mathrm{E}-06 \quad 1.553 \mathrm{E}-140.0$ $\begin{array}{lllll}.0 & 0.0 & 0.0 & 0.0 & 0.0\end{array}$

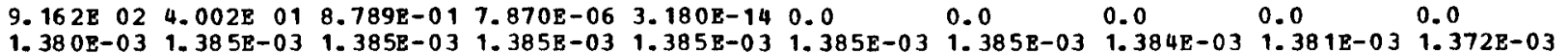
$\begin{array}{lllllllllll}4.630 \mathrm{E} & 00 & 2.331 \mathrm{E} & 0 & 1.174 \mathrm{E} & 00 & 1.498 \mathrm{E}-01 & 4.849 \mathrm{E}-03 & 5.321 \mathrm{E}-09 & 7.362 \mathrm{E}-30 & 0.0\end{array}$ $8.174 \mathrm{E} 02$ $3.835 \mathrm{E} 02$ $1.937 \mathrm{E} 022.465 \mathrm{E} 017.9778-018.753 \mathrm{E}-071.211 \mathrm{E}-270.0$ $6.593 \mathrm{E} 00 \quad 5.153 \mathrm{E} 00 \quad 3.998 \mathrm{E} 001.866 \mathrm{E} 00 \quad 5.244 \mathrm{E}-01 \quad 3.272 \mathrm{E}-03 \quad 6.250 \mathrm{E}-110.0$ 0.0 0.0
0.0
1.34 0.0 0.0 $1.256 \mathrm{E}-03$ $\begin{array}{llllllll} & \\ 0\end{array}$ $\begin{array}{llllll} & \end{array}$

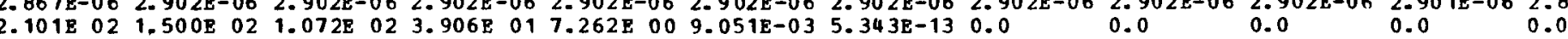
2.668E-05 2.672E-05 2.672E-0 5 2.672E-05 2.672E-05 2.672E-05 2.672E-05 2.671E-05 2.671E-05 2.669 E-05 2.664E-05 2.648E-05 年

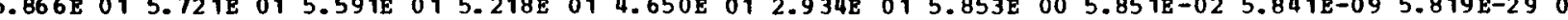
$1.051 \mathrm{E} 02$ 4. 316E 01 1.772E $011.226 \mathrm{E}$ 00 $1.431 \mathrm{E}-02 \quad 2.648 \mathrm{E}-100.0$ $0.0 \quad 0.0$ 1. $221 \mathrm{E} 03$ 4. $987 \mathrm{E} 02 \quad 2.047 \mathrm{E} 02$ 1.417 01 1.654E-01 3.059E-09 0.0 0.000 .0 $.722 \mathrm{E} 006.180 \mathrm{E}$ OO $4.745 \mathrm{E}$ OO $2.148 \mathrm{E}$ 0O $5.730 \mathrm{E}-012.907 \mathrm{E}-03$ 2.6948-11 0.0

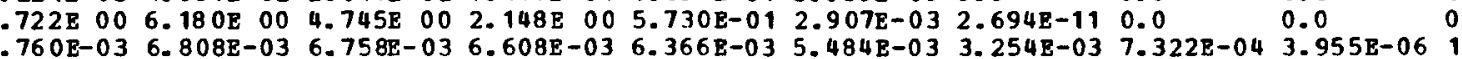

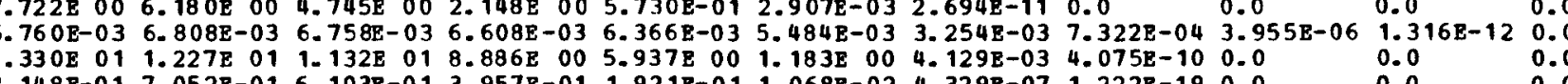

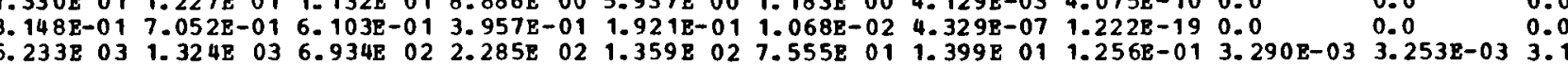
$\begin{array}{ll}.0 & 0.0 \\ 0 & 0.0\end{array}$ 
Table 2.11. Watts of heavy-element isotopes in a BWR assembly
DISC HARGE
1. $Y R$
2. $Y R$
5. $\mathbf{Y R}$
10. YR
30. IR 100. YR
300. IR
1000. TR 3000. PR 10000. IR 30000. TR

BI2 14

PO2 10

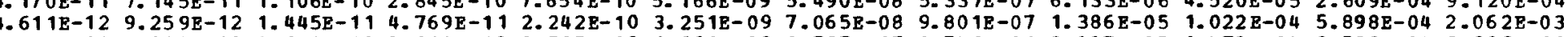
$\begin{array}{lllllll} & \end{array}$

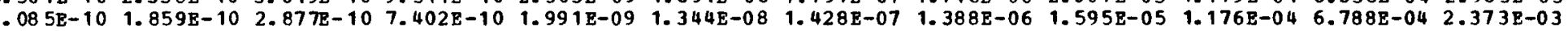

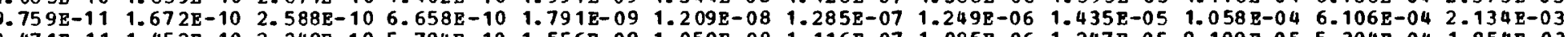
1. 1.

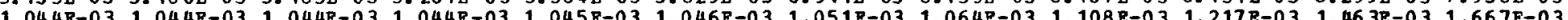

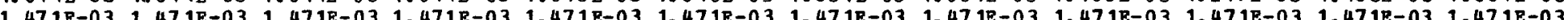

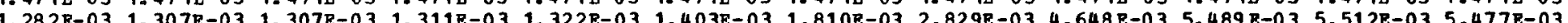

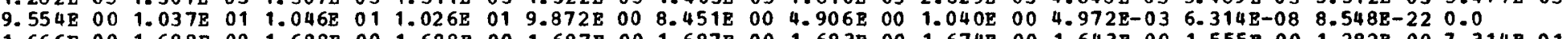

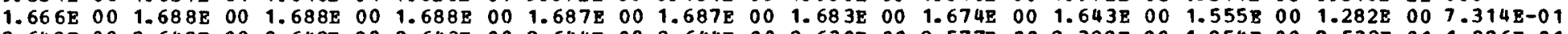

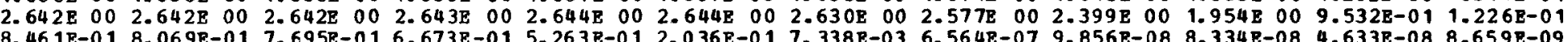

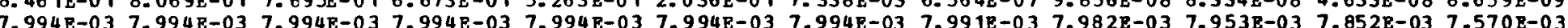

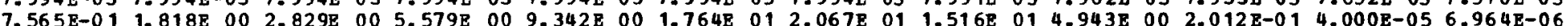
8.205E-02 8.214E-02 8.213E-02 8.211E-02 8.207E-02 8.192E-02 8. 1408-02 7.994E-02 7.503E-02 6.259E-02 3.319E-02 5.421E-03 $2.285 \mathrm{E} 02$ 4.866E 01 1.031E 01 1.219E-01 2.400E-02 2.186E-02 1.589E-02 6.382E-03 2.622E-04 2.877E-08 $3.942 \mathrm{E}-22 \quad 0.0$

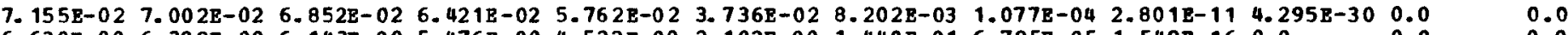

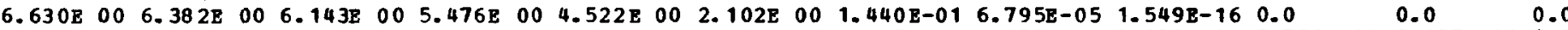

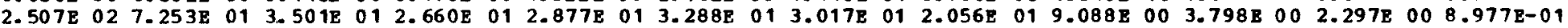

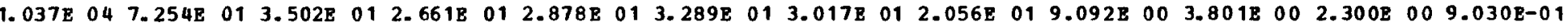


Table 2.12. Photon spectrum for light-element isotopes in a BWR assembly

12 GROUP PHOTON RELEASE RATES, PHOTONS/SECOND

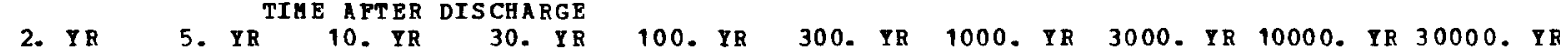

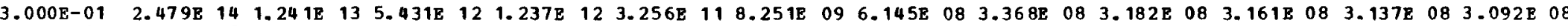
$\begin{array}{llllllllllllllllllllllllll}6.300 \mathrm{E}-01 & 3.993 \mathrm{E} & 15 & 5.775 \mathrm{E} & 13 & 1.109 \mathrm{E} & 13 & 4.785 \mathrm{E} & 12 & 1.486 \mathrm{E} & 12 & 6.205 \mathrm{E} & 10 & 5.615 \mathrm{E} & 09 & 5.403 \mathrm{E} & 09 & 5.269 \mathrm{E} & 09 & 4.911 \mathrm{E} & 09 & 3.850 \mathrm{E} & 09 & 1.924 \mathrm{E} & 09\end{array}$

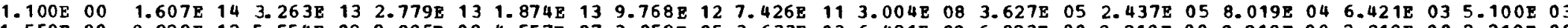

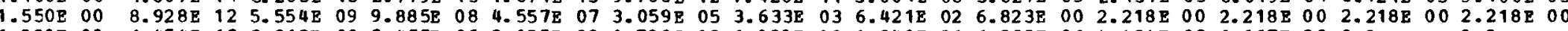

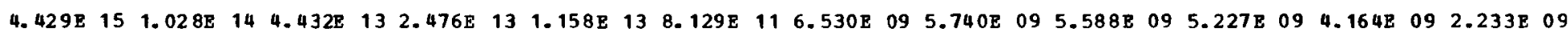

MEV/SEC

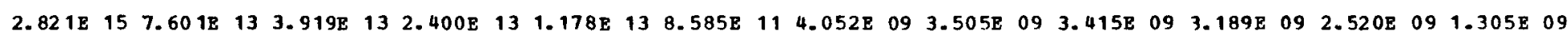
12 GROUP SPECIPIC RNERGY RELEASE RATES, MEV/RATT-SEC

EMEAN
DISCHARG E
1. IR
2. I B
5. $Y R$
AFTER
10 . YR
30. I R

100. YR

300. $\mathrm{IB}$

1000. $Y$

3000. IR 10000. YR 30000 . IR

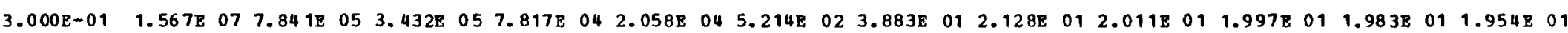

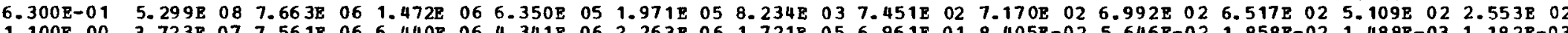

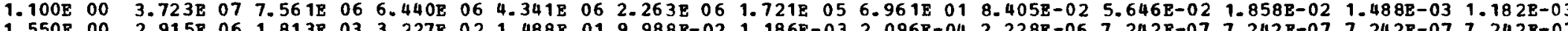
$.915 \mathrm{E} 061.813 \mathrm{E}$ (1. 05

750000

$3.250 \mathrm{E} 00$

$3.700 \mathrm{~B} 00$

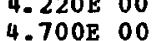
$130 \mathrm{~B} 069.731 \mathrm{E}-15 \quad 4.900 \mathrm{E}-156.255 \mathrm{E}-1622.029 \mathrm{E}-17 \quad 2.226 \mathrm{E}-23 \quad 3.080 \mathrm{~B}-440.0$

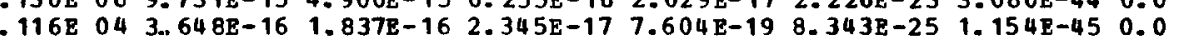

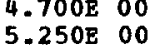
$.071 \mathrm{E} 010.0$ 2.0508000 .0

1.8
0.0
0.0

TOTAL

$0.0 \quad 0.0$

0.0

0.0

0.0
0.0

Ga 
Table 2.13. Photon spectrum for fission-product isotopes in a BWR assembly

12 GROUP PHOTON RELEASE RATES, PHOTONS/SECOND

EHEAN

DI SCHARGE

1. $\mathrm{YR}$

2. $\mathrm{YR}$

5. $\mathrm{YR}$ TIME 10 . YR

30. YR

100. YR 300. YR 1000. YR 3000. YR 10000. YR 30000. YR

$3.000 \mathrm{E}-01$ 6. $300 \mathrm{E}-01$ 1. $100 \mathrm{E} 00$

$\begin{array}{llllllllllllllllllllllll} & & \end{array}$

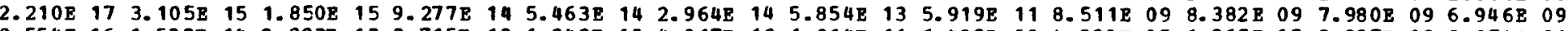

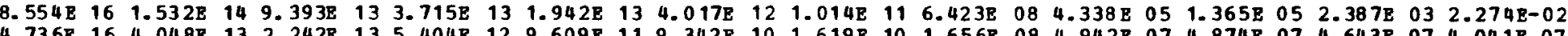

$2.380 \mathrm{E} 00$

$2.750 \mathrm{E} 00$

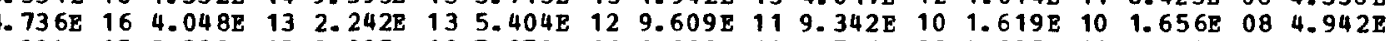
$.994 \mathrm{E} 15$ 2.332E 13 9.885E $127.872 \mathrm{E} \quad 11$ 1.808E 10 2.746E 09 4.885E 08 3.519E $06 \quad 1.115 \mathrm{E}$

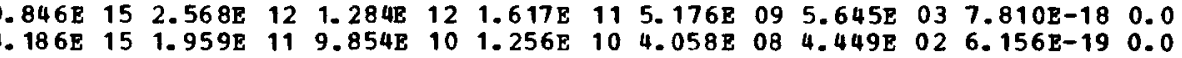

. $2500 \mathrm{E} 00$

$4.220 \mathrm{E} 00$

$4.700 E 00$ 1738 15 6.1828 E $4.025 E 140.0$ $1.136 \mathrm{E} 15 \mathrm{0.0}$ $113 \mathrm{E} 09 \quad 3.96 \mathrm{E}$ 10 $4.058 \mathrm{E} \quad 08$ 4.449E $02 \quad 6.156 \mathrm{E}-190.0$

$5.250 \mathrm{E} 00$ $1.293 \mathrm{E} 140.0$ 0.0

TOTAL $\begin{array}{lll}0.0 & 0.0 & 0.0\end{array}$ 1.2868
0.0
0.0 $\begin{array}{llll}0.0 & 0.0 & 0.0 & 0.0\end{array}$ 0.0
0.0
0.0 $4.874 \mathrm{E} 074.643 \mathrm{E} 07 \quad 4.041 \mathrm{~B} 07$

EV/SEC

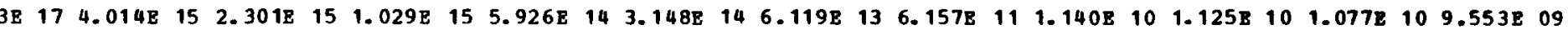
$\begin{array}{lllllllllllllllllllllllll}4.290 \mathrm{E} & 17 & 2.447 \mathrm{E} & 15 & 1.423 \mathrm{E} & 15 & 6.531 \mathrm{E} & 14 & 3.749 \mathrm{E} & 14 & 1.956 \mathrm{E} & 14 & 3.778 \mathrm{E} & 13 & 3.808 \mathrm{E} & 11 & 6.291 \mathrm{E} & 09 & 6.202 \mathrm{E} & 09 & 5.924 \mathrm{E} & 09 & 5.209 \mathrm{E} & 09\end{array}$ 12 GROOP SPECIFIC ENERGY RELEASE RATES, HEV/MATT-SEC

EHEAN
DI SCHARGE
1. $\mathrm{YR}$
2. YR
5. $Y R \quad$ TIHE APTER
SCHARGE
30. IR

100. IR 300. IR 1000. YR 3000. YR 10000. YR 30000. IR

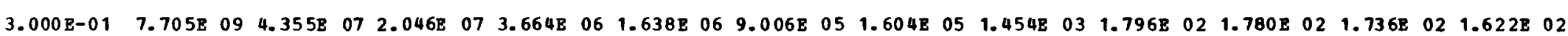

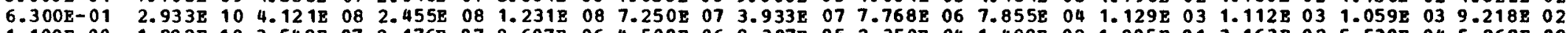

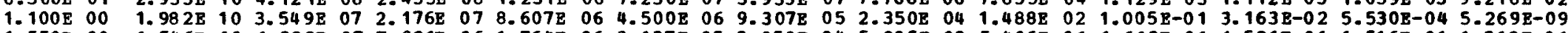

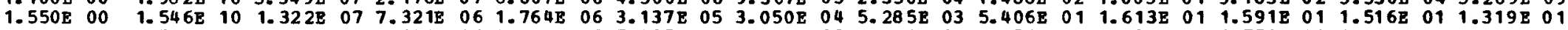
$3.770 E$ O9 9.774E 06 4.143E 06 3.300E 05 7.577E 03 1.151E 03 2.048E 02 1.475E 00 4. 936E 09 1. 287E 06 6.438E 05 8. 104E 04 2.595E 03 2.830E-03 $3.915 \mathrm{E}-24 \quad 0.0$

$3.250 \mathrm{E} \quad 00$ $3.700 \mathrm{E}$
4.20 $4.700 \mathrm{E} 00$ 3. $5668-250.0$ $4.911 \mathrm{E} 094.232 \mathrm{E} 03$ 2. $131 \mathrm{E} 03$ $2.720 \mathrm{E} 028.804 \mathrm{E} 009.660 \mathrm{E}-061.337 \mathrm{E}-260.0$ $3.137 \mathrm{~B} 080.0$ 1.0108090 .0 0.0 $5.387 \mathrm{E} 080.0$ 0.0
0.0 0.0 0.0 0.0
0.0

0.0 0.0

0.0

0.0 0.0 0.0 0.0 0.0
0.0
0.0

0.0 0.0 $0.0 \quad 0.0$ $\begin{array}{ll}0.0 & 0.0 \\ 0.0 & 0.0\end{array}$ 0.0

TOTAL

$\begin{array}{lllllllllllllllllllllllllll}9.037 \mathrm{E} & 10 & 5.155 \mathrm{E} & 08 & 2.998 \mathrm{E} & 08 & 1.376 \mathrm{E} & 08 & 7.896 \mathrm{E} & 07 & 4.120 \mathrm{E} & 07 & 7.957 \mathrm{E} & 06 & 8.021 \mathrm{E} & 04 & 1.325 \mathrm{E} & 03 & 1.306 \mathrm{E} & 03 & 1.248 \mathrm{E} & 03 & 1.097 \mathrm{E} & 03\end{array}$

GAM POH

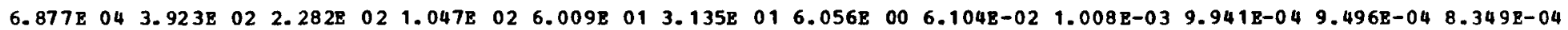


18 GROOP PHOTON RELEASE RATES, PHOTONS/SECOND

EHEAN
DI SC HARG E
1. $\mathrm{YR}$
2. $\mathrm{YR}$
5. $\mathrm{YR}$
TISE AFTER
10. YR
SCHARGE
30. Y R

100. IR

300. IR 1000. IR 3000. IR 10000. IR 30000. IR

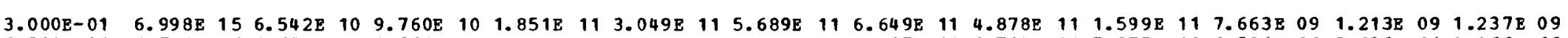

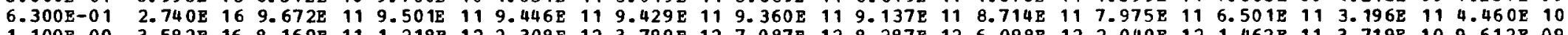
$\begin{array}{llllllllllllllllllllllllll}1.100 \mathrm{E} & 00 & 3.582 \mathrm{E} & 16 & 8.169 \mathrm{E} & 11 & 1.218 \mathrm{E} & 12 & 2.308 \mathrm{E} & 12 & 3.799 \mathrm{E} & 12 & 7.087 \mathrm{E} & 12 & 8.287 \mathrm{E} & 12 & 6.098 \mathrm{E} & 12 & 2.040 \mathrm{E} & 12 & 1.462 \mathrm{E} & 11 & 3.719 \mathrm{E} & 10 & 9.612 \mathrm{E} & 09\end{array}$

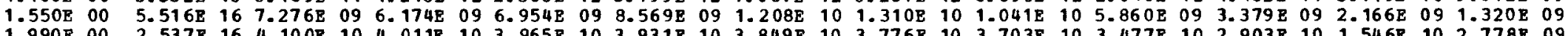

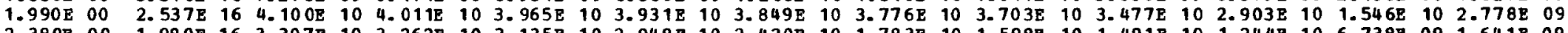

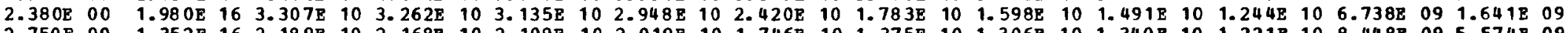

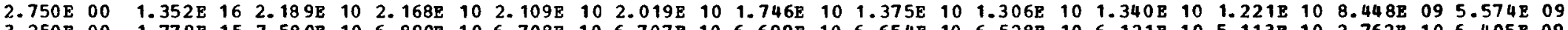

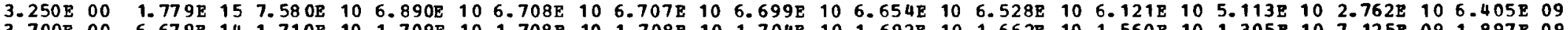

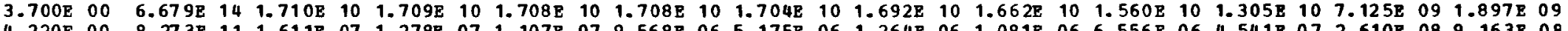

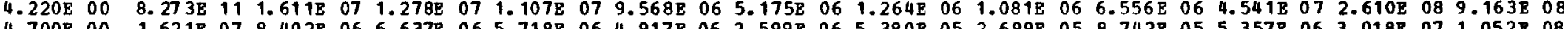

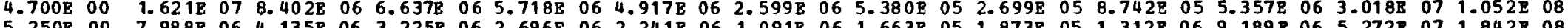
$5.250 \mathrm{E} 00 \mathrm{~F}$ 3.00E $4.000 \mathrm{E}-0202.31 \mathrm{E} 061.195 \mathrm{E}$.

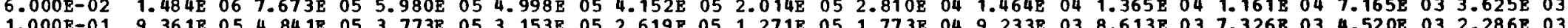

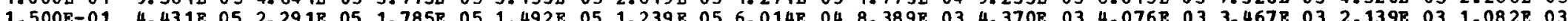

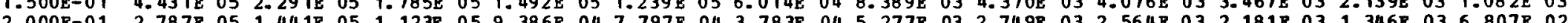

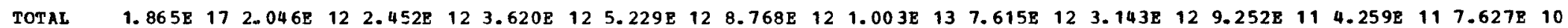

MEV/SEC

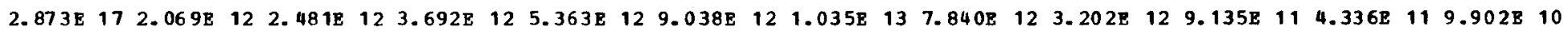

18 GROOP SPECI PIC ENERGY RELEASE RATES, HEV/RATT-SEC

EHEAN $6.300 \mathrm{E}-01$ $1.100 \mathrm{E} 00$ $1.550 \mathrm{E}$ OO $\begin{array}{ll}1.990 E & 00 \\ 2.380 \mathrm{E} & 0 O\end{array}$ $2.380 \mathrm{E}$
$2.750 \mathrm{E}$ $3.250 \mathrm{E} 00$ $3.700 E 00$
$4.220 E$ $\begin{array}{ll}4.220 \mathrm{E} & 00 \\ 4.700 \mathrm{E} & 00\end{array}$ $4.700 \mathrm{E} 00$
$5.250 \mathrm{E}$ 00 3. $000 \mathrm{E}-02$ $3.000 \mathrm{E}-02$
$4.000 \mathrm{E}-02$ $4.000 \mathrm{E}-02$
$6.000 \mathrm{E}-02$ 1.000E-01 $1.500 \mathrm{E}-01$

TOTAL

GAM PON

1. $\mathrm{YR}$

2. $\mathrm{YR}$

5. $I$

$T$

E ATER DISCHARGE
10. YR TR

100. YR 300. YR 1000 . I 3000 . YR 10000, IR 30000 . IR

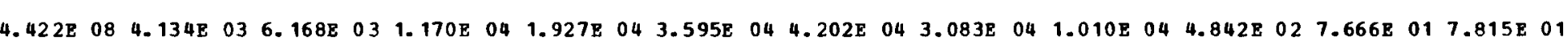

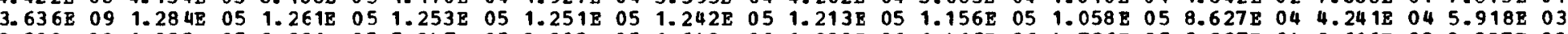
$\begin{array}{llllllllllllll} & \end{array}$

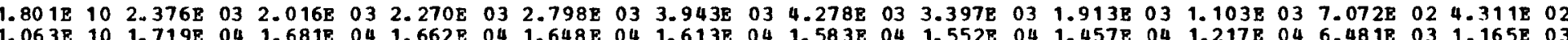

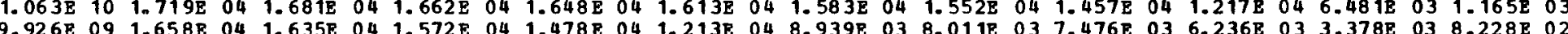

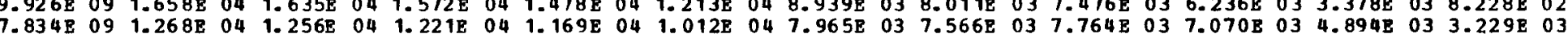

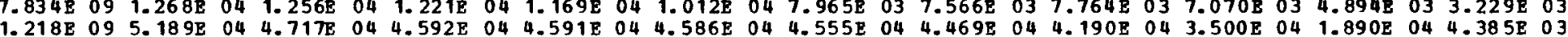

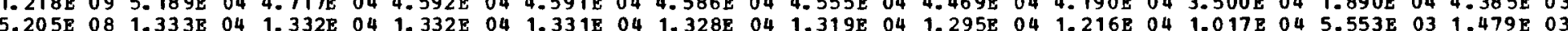

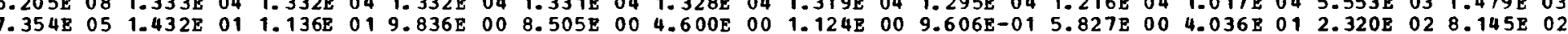

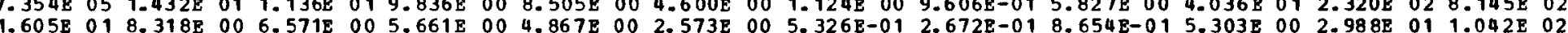

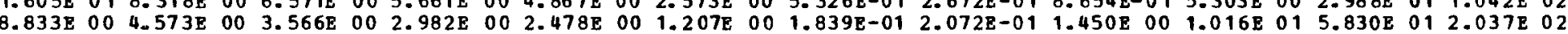
4.032E-02 4. $437 \mathrm{E}-02 \quad 6.650 \mathrm{E}-02$ 1.122E-01 $1.484 \mathrm{E}-01$ 1.397E-01 7.015E-02 $1.042 \mathrm{E}-02 \quad 4.035 \mathrm{E}-04 \quad 1.442 \mathrm{E}-03 \quad 7.348 \mathrm{E}-03 \quad 2.533 \mathrm{E}-02$

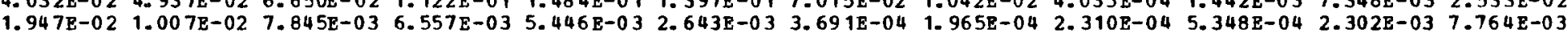
$1.876 \mathrm{E}-02 \quad 9.698 \mathrm{E}-03 \quad 7.558 \mathrm{E}-03 \quad 6.317 \mathrm{E}-03$ 5.247E-03 $2.546 \mathrm{E}-03 \quad 3.551 \mathrm{E}-04$ 1.850E-04 $1.726 \mathrm{E}-04 \quad 1.468 \mathrm{E}-04 \quad 9.055 \mathrm{E}-05$ 4.581E-05

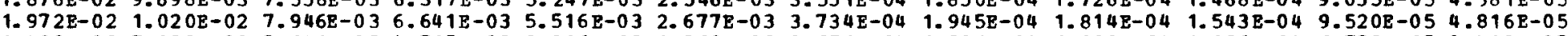
$1.400 \mathrm{E}-02 \quad 7.238 \mathrm{E}-03 \quad 5.641 \mathrm{E}-03 \quad 4.715 \mathrm{E}-03 \quad 3.916 \mathrm{E}-03 \quad 1.900 \mathrm{E}-03 \quad 2.651 \mathrm{E}-04$ 1. $381 \mathrm{E}-04 \quad 1.288 \mathrm{E}-04 \quad 1.096 \mathrm{E}-04 \quad 6.759 \mathrm{E}-05 \quad 3.419 \mathrm{E}-05$

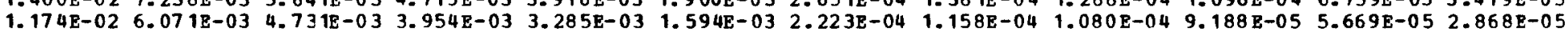

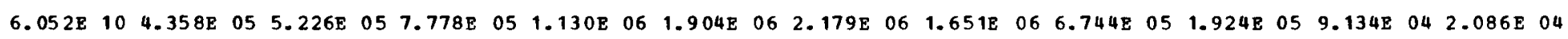
$\begin{array}{lllllllllllllll}4.606 \mathrm{E} & 04 & 3.317 \mathrm{E}-01 & 3.977 \mathrm{E}-01 & 5.919 \mathrm{E}-01 & 8.597 \mathrm{E}-01 & 1.449 \mathrm{E} & 00 & 1.658 \mathrm{E} & 00 & 1.257 \mathrm{E} & 00 & 5.132 \mathrm{E}-01 & 1.464 \mathrm{E}-01 & 6.951 \mathrm{E}-02 \quad 1.587 \mathrm{E}-02\end{array}$ 
Table 2.15. Total curles in BWR assembly as a function of age

\begin{tabular}{ccccc}
\hline $\begin{array}{c}\text { Years after } \\
\text { discharge }\end{array}$ & $\begin{array}{c}\text { Activation } \\
\text { products }\end{array}$ & $\begin{array}{c}\text { Heavy } \\
\text { elements }\end{array}$ & $\begin{array}{c}\text { Fission } \\
\text { products }\end{array}$ & Total \\
\hline 0 & $1.175 \times 10^{5}$ & $5.474 \times 10^{6}$ & $1.998 \times 10^{7}$ & $2.557 \times 10^{7}$ \\
1 & $3.267 \times 10^{3}$ & $2.146 \times 10^{4}$ & $3.149 \times 10^{5}$ & $3.396 \times 10^{5}$ \\
2 & $1.380 \times 10^{3}$ & $1.955 \times 10^{4}$ & $1.729 \times 10^{5}$ & $1.938 \times 10^{5}$ \\
5 & $5.962 \times 10^{2}$ & $1.687 \times 10^{4}$ & $6.924 \times 10^{4}$ & $8.671 \times 10^{4}$ \\
10 & $2.552 \times 10^{2}$ & $1.354 \times 10^{4}$ & $4.666 \times 10^{4}$ & $6.046 \times 10^{4}$ \\
30 & $3.910 \times 10^{1}$ & $5.899 \times 10^{3}$ & $2.673 \times 10^{4}$ & $3.267 \times 10^{4}$ \\
100 & $1.171 \times 10^{1}$ & $1.094 \times 10^{3}$ & $5.044 \times 10^{3}$ & $6.150 \times 10^{3}$ \\
300 & 3.048 & $6.282 \times 10^{2}$ & $5.414 \times 10^{1}$ & $6.854 \times 10^{2}$ \\
1,000 & $6.367 \times 10^{-1}$ & $2.834 \times 10^{2}$ & 3.337 & $2.874 \times 10^{2}$ \\
3,000 & $5.671 \times 10^{-1}$ & $1.234 \times 10^{2}$ & 3.284 & $1.273 \times 10^{2}$ \\
10,000 & $4.408 \times 10^{-1}$ & $7.506 \times 10^{1}$ & 3.218 & $7.872 \times 10^{1}$ \\
30,000 & $3.236 \times 10^{-1}$ & $2.973 \times 10^{1}$ & 3.039 & $3.309 \times 10^{1}$ \\
\hline
\end{tabular}

Table 2.16. Total watts of thermal power in BWR assembly as a function of age

\begin{tabular}{rlccc}
\hline $\begin{array}{c}\text { Years after } \\
\text { discharge }\end{array}$ & $\begin{array}{c}\text { Activation } \\
\text { products }\end{array}$ & $\begin{array}{c}\text { Heavy } \\
\text { elements }\end{array}$ & $\begin{array}{c}\text { Fission } \\
\text { products }\end{array}$ & Total \\
\hline 0 & $6.080 \times 10^{2}$ & $1.037 \times 10^{4}$ & $2.378 \times 10^{5}$ & $2.488 \times 10^{5}$ \\
1 & $1.430 \times 10^{1}$ & $7.254 \times 10^{1}$ & $1.327 \times 10^{3}$ & $1.414 \times 10^{3}$ \\
2 & 7.459 & $3.502 \times 10^{1}$ & $6.940 \times 10^{2}$ & $7.365 \times 10^{2}$ \\
5 & 4.500 & $2.661 \times 10^{1}$ & $2.287 \times 10^{2}$ & $2.598 \times 10^{2}$ \\
10 & 2.189 & $2.878 \times 10^{1}$ & $1.359 \times 10^{2}$ & $1.669 \times 10^{2}$ \\
30 & $1.675 \times 10^{-1}$ & $3.289 \times 10^{1}$ & $7.556 \times 10^{1}$ & $1.086 \times 10^{2}$ \\
100 & $6.370 \times 10^{-3}$ & $3.017 \times 10^{1}$ & $1.399 \times 10^{1}$ & $4.417 \times 10^{1}$ \\
300 & $2.799 \times 10^{-3}$ & $2.056 \times 10^{1}$ & $1.256 \times 10^{-1}$ & $2.069 \times 10^{1}$ \\
1,000 & $1.802 \times 10^{-3}$ & 9.092 & $3.293 \times 10^{-3}$ & 9.097 \\
3,000 & $1.693 \times 10^{-3}$ & 3.801 & $3.254 \times 10^{-3}$ & 3.806 \\
10,000 & $1.420 \times 10^{-3}$ & 2.300 & $3.140 \times 10^{-3}$ & 2.305 \\
30,000 & $9.968 \times 10^{-4}$ & $9.030 \times 10^{-1}$ & $2.840 \times 10^{-3}$ & $9.068 \times 10^{-1}$
\end{tabular}



Table 2.17. Spontaneous fission neutron source in a BWR assembly
(neutrons $/ \mathrm{sec}$ )

DISCHARGE $\quad$ 1. YR $\quad$ 2. YR $\quad$ 5. YR 10 . YR 30 . YR 100. YR 300. YR 1000. YR 3000. YR 10000. YR 30000. YR 4. $282 \mathrm{E} \quad 04 \quad 4.646 \mathrm{E} \quad 04 \quad 4.687 \mathrm{E} \quad 04 \quad 4.599 \mathrm{E} \quad 04 \quad 4.424 \mathrm{E} \quad 04 \quad 3.787 \mathrm{E} \quad 04 \quad 2.199 \mathrm{E} \quad 04 \quad 4.661 \mathrm{E} \quad 03 \quad 2.228 \mathrm{E} \quad 01 \quad 2.830 \mathrm{E}-04 \quad 3.831 \mathrm{E}-18 \quad 0.0$

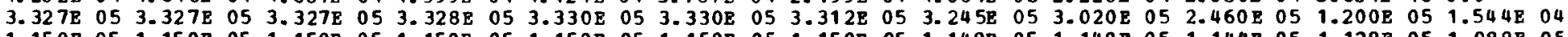

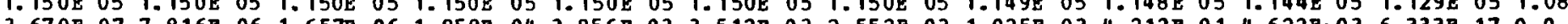

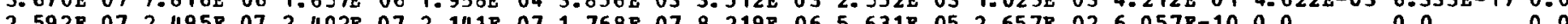

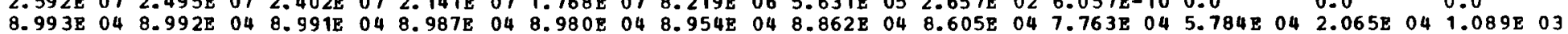

$\begin{array}{llllllllllllllllllllllllllll}6.320 \mathrm{E} & 07 & 3.335 \mathrm{E} & 07 & 2.626 \mathrm{E} & 07 & 2.201 \mathrm{E} & 07 & 1.826 \mathrm{E} & 07 & 8.798 \mathrm{E} & 06 & 1.123 \mathrm{E} & 06 & 5.315 \mathrm{E} & 05 & 4.946 \mathrm{E} & 05 & 4.183 \mathrm{E} & 05 & 2.537 \mathrm{E} & 05 & 1.255 \mathrm{E} & 05 \\ 6.320 \mathrm{E} & 07 & 3.335 \mathrm{E} & 07 & 2.626 \mathrm{E} & 07 & 2.201 \mathrm{E} & 07 & 1.826 \mathrm{E} & 07 & 8.798 \mathrm{E} & 06 & 1.123 \mathrm{E} & 06 & 5.315 \mathrm{E} & 05 & 4.946 \mathrm{E} & 05 & 4.183 \mathrm{E} & 05 & 2.537 \mathrm{E} & 05 & 1.255 \mathrm{E} & 05\end{array}$ ACTOA

Table 2.18. Alpha, n neutron source in a BWR assembly (neutrons/sec)

DISCHARGE 1 1. IR 2 2. YR 5 5. YR 10 . YR 30 . YR 100. YR 300. IR 1000. TR 3000. IR 10000. YR 30000. YR

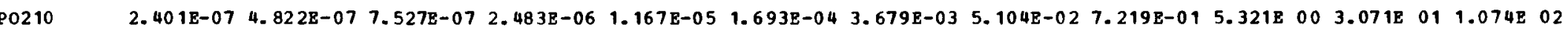
P0213

P0214

P0218

คT217

RA222

PR221

RA226

AC225

TH230

0234

0236

0238
$\times \quad 237$

P0238

P0239

$\mathrm{P} 0240$

P0241

P0242

A 241

A 1243

C) 242

Cu244

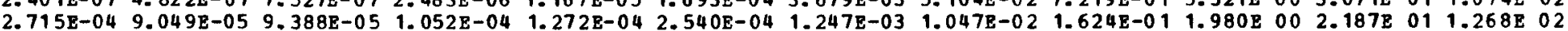
4. $859 \mathrm{E}-05$ 3.246E-05 5.023E-05 $1.292 \mathrm{E}-04 \quad 3.476 \mathrm{E}-04 \quad 2.347 \mathrm{E}-03 \quad 2.494 \mathrm{E}-02 \quad 2.424 \mathrm{E}-012.786 \mathrm{E} \quad 00 \quad 2.053 \mathrm{E} \quad 01 \quad 1.185 \mathrm{E} \quad 02 \quad 4.143 \mathrm{E} \quad 02$ 8. $199 \mathrm{E}-06 \quad 1.405 \mathrm{E}-05 \quad 2.174 \mathrm{E}-05 \quad 5.593 \mathrm{E}-05 \quad 1.505 \mathrm{E}-04 \quad 1.016 \mathrm{E}-03 \quad 1.079 \mathrm{E}-02 \quad 1.049 \mathrm{E}-01 \quad 1.206 \mathrm{E} \quad 00 \quad 8.887 \mathrm{E} \quad 00 \quad 5.129 \mathrm{E} \quad 019.793 \mathrm{E} \quad 02$

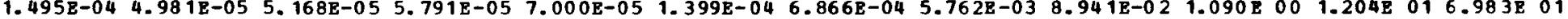
5. 570E-06 9.544E-06 1.477E-05 3.800E-05 1.022E-04 6.900E-04 7.333E-03 7.129E-02 8.192E-01 6.038E 00 3.485E $01 \quad 1.218 \mathrm{E} \quad 02$ $9.670 \mathrm{E}-05 \quad 3.223 \mathrm{E}-05 \quad 3.344 \mathrm{E}-05 \quad 3.747 \mathrm{E}-05 \quad 4.529 \mathrm{E}-05$ 9.049E-05 $4.442 \mathrm{E}-04 \quad 3.728 \mathrm{E}-03 \quad 5.785 \mathrm{E}-02 \quad 7.053 \mathrm{E}-01 \quad 7.788 \mathrm{E} \quad 00 \quad 4.518 \mathrm{E} \quad 01$ 3.329E-06 5.709E-06 8.835E-06 2.273E-05 6.115E-05 4.127E-04 4.386E-03 4.264E-02 4.900 E-01 3.611E 00 2.085E 01 7.287E 01

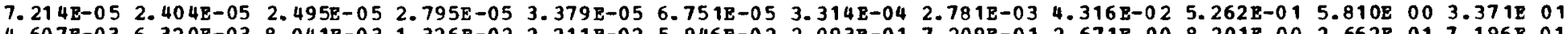
$4.607 \mathrm{E}-03$ 6.320E-03 8.041E-03 $1.326 \mathrm{E}-02$ 2.211E-02 5.946E-02 2.093E-01 7.209E-01 2.671E 00 8.201E 00 2.662E 01 7.196E 01 $\begin{array}{llllllllllllllllllllllll}2.110 E & 02 & 2.120 E & 02 & 2.131 E & 02 & 2.162 E & 02 & 2.212 E & 02 & 2.395 E & 02 & 2.852 E & 02 & 3.350 E & 02 & 3.479 E & 02 & 3.465 E & 02 & 3.411 E & 02 & 3.262 E & 02\end{array}$

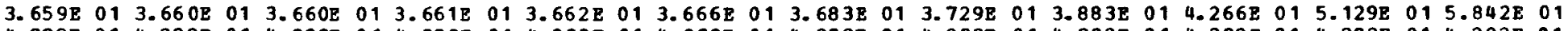

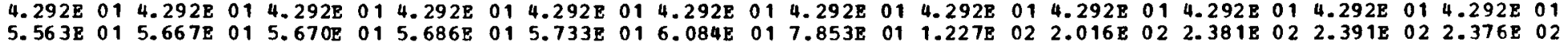

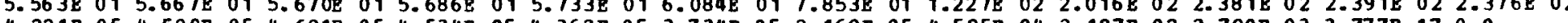

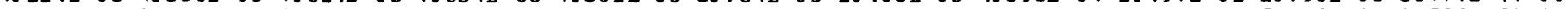

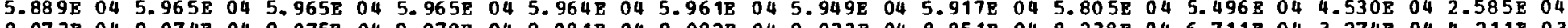

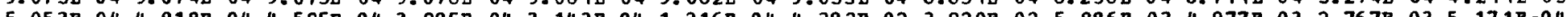
5. 53 E 04 .

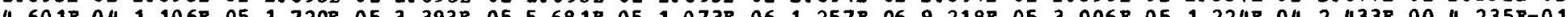

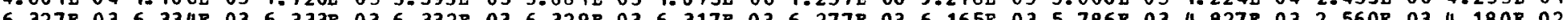

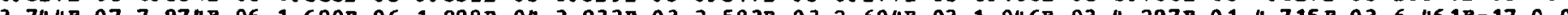

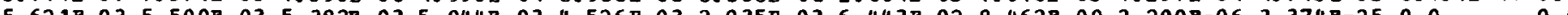

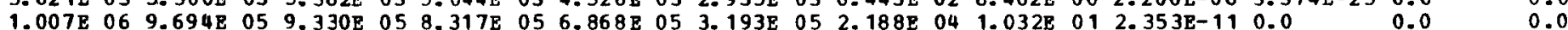


Table 2.19. Total grams charged to a PWR fuel assembly ${ }^{a}$

\begin{tabular}{|c|c|}
\hline $\begin{array}{c}\text { Elements or } \\
\text { Isotopes }\end{array}$ & $\begin{array}{l}\text { Grams } \\
\text { charged }\end{array}$ \\
\hline $\mathrm{C}$ & $7.353 \times 10^{1}$ \\
\hline $\mathbf{N}$ & $5.302 \times 10^{1}$ \\
\hline 0 & $6.214 \times 10^{4}$ \\
\hline Si & $2.107 \times 10^{2}$ \\
\hline $\mathbf{P}$ & $1.485 \times 10^{2}$ \\
\hline $\mathrm{Cr}$ & $5.081 \times 10^{3}$ \\
\hline Mn & $4.003 \times 10^{2}$ \\
\hline $\mathrm{Fe}$ & $1.460 \times 10^{4}$ \\
\hline $\mathrm{Ni}$ & $5.740 \times 10^{3}$ \\
\hline $\mathrm{Zr}$ & $1.062 \times 10^{5}$ \\
\hline $\mathrm{Nb}$ & $3.280 \times 10^{2}$ \\
\hline Mo & $1.818 \times 10^{2}$ \\
\hline Sn & $1.737 \times 10^{3}$ \\
\hline $235 \mathrm{U}$ & $1.476 \times 10^{4}$ \\
\hline $238 \mathrm{U}$ & $4.465 \times 10^{5}$ \\
\hline
\end{tabular}

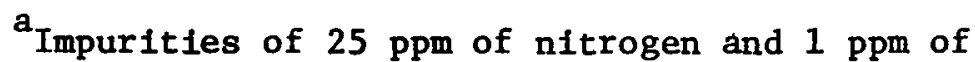
lithium, in addition to others, are found in the fuel. 
Table 2.20. Grams of fission-product elements in a PWR assembly

1. $\mathrm{YR}$

2. $\mathbf{Y R}$

5. $\mathbf{R}$

10. $\mathrm{YR}$

30. 78

100. YR

300. IR

1000 . 18

3000

0.0

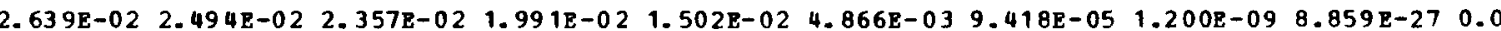
0.0

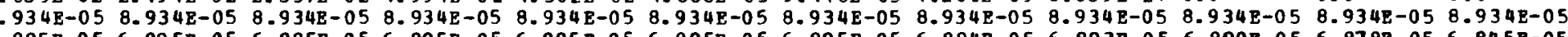

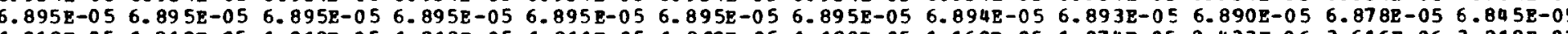
$1.212 \mathrm{E}-05 \quad 1.212 \mathrm{E}-05 \quad 1.212 \mathrm{E}-05 \quad 1.212 \mathrm{E}-05 \quad 1.211 \mathrm{E}-05 \quad 1.2 \mathrm{C8E}-05 \quad 1.198 \mathrm{E}-05 \quad 1.169 \mathrm{E}-05 \quad 1.074 \mathrm{E}-05 \quad 8.433 \mathrm{E}-06 \quad 3.616 \mathrm{E}-06 \quad 3.218 \mathrm{E}-07$ $7.299 \mathrm{E}-130.0$ $\begin{array}{lll}0.0 & 0.0 & 0.0 \\ 0.0 & 0.0 & 0.0\end{array}$ 0.0 0.0 0.0 0.0 0.0 0.0 0.0 0.0 0.0
0.0 0.0 0.0 0.0 0.0 0.0 0.0

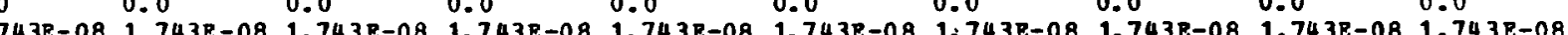
$1.794 \mathrm{E}-05$ 4.093E-07 4.093E-07 4.093E-07 4.093E-07 4.093E-07 4.093E-07 4.093E-07 4.093E-07 4.093E-07 4.093E-07 4.093E-07 3. 047E-01 3.04 5E-01 3.045E-01 3.045E-01 3.045E-01 3.045E-01 3.045E-01 3.045E-01 3.045E-01 3.045E-01 3.045E-01 3.045E-01

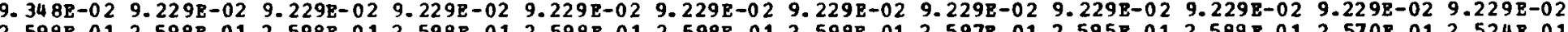
2.598E 01 2.598E 01 2.598E $012.598 \mathrm{E} 01$ 2.598E 01 2.598E 01 2.598E 01 2.597E 01 2.595E 01 2.589E 01 2.570E $012.524 \mathrm{E} \quad 01$

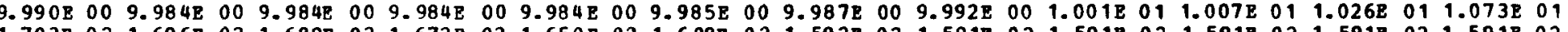

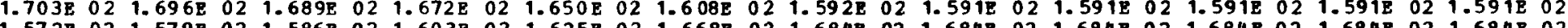

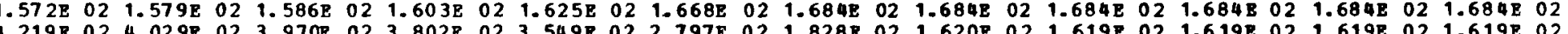

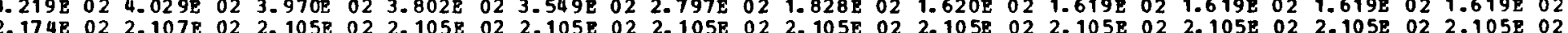

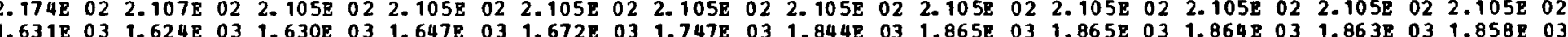

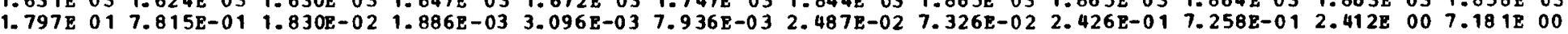

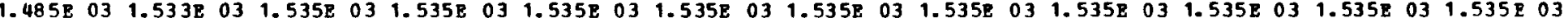

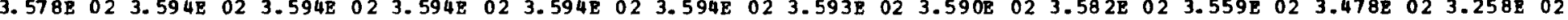

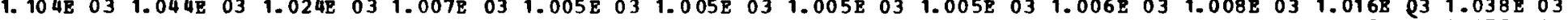

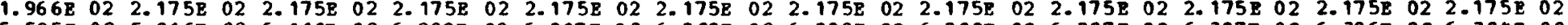

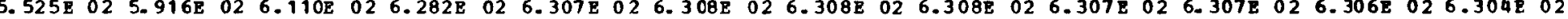

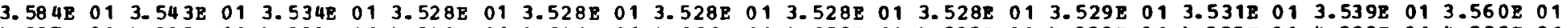

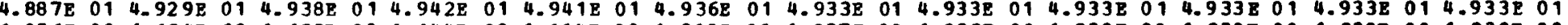

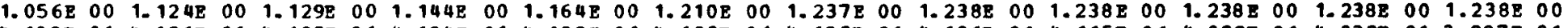

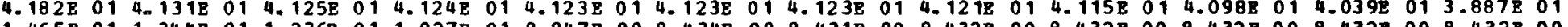

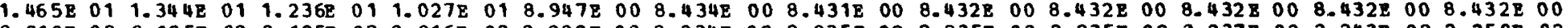

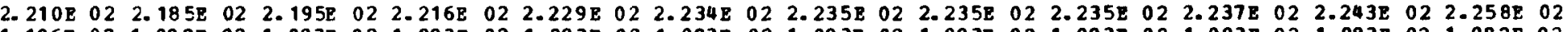

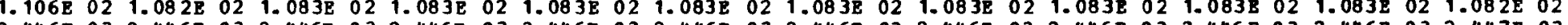
$2.446 \mathrm{E} 03$ 2.446E 03 2.446E 03 2.446E 03 2.446E 03 2.446E 03 2.446E 03 $2.446 \mathrm{E} 03$ 2.446E 03 2.446E 03 2.446E 03 2.447E 03

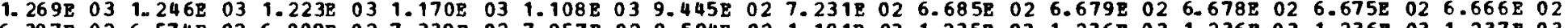
6.

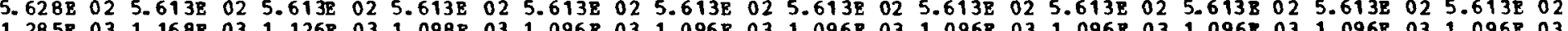

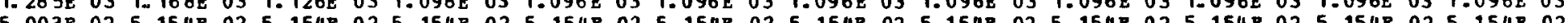

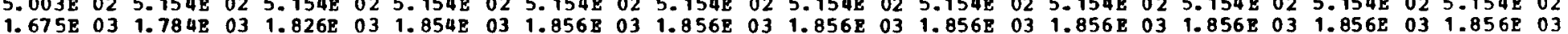
0.0 0.0 0.0

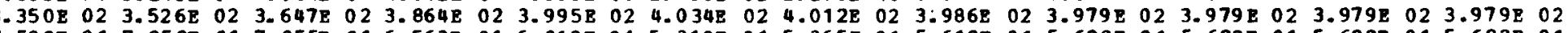

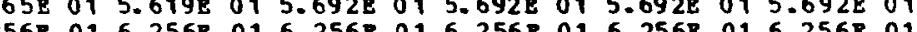

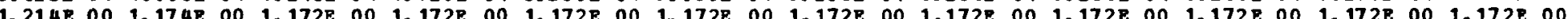

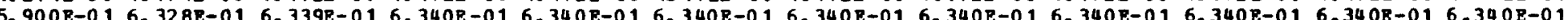
$6.303 \mathrm{~B}-02$ 6.2968-02 6.2968-02 6.2968-02 6.2968-02 6.2958-02 6.2938-02 6.286B-02 6.2698-02 6.2448-02 $6.233 \mathrm{~B}-026.233 \mathrm{~B}-02$

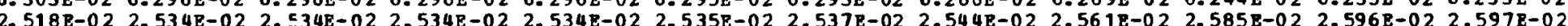

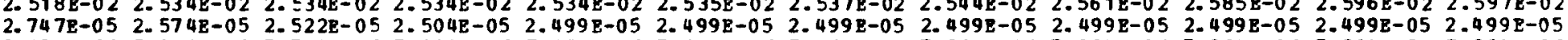
3. 972E-06 7.2108-06 7.728E-06 7.909E-06 7.955E-06 7.964E-06 7.964E-06 7.964E-06 7.964 E-06 7.964E-06 7.964E-06 7.964E-06

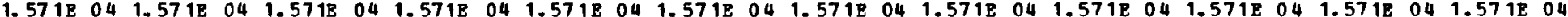



2. $\mathrm{YR}$
5. YR

DISCHARG E

10. $\mathrm{IR}$

30. $\mathrm{YB}$

in a PWR
100. IR

sembly

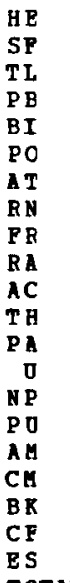

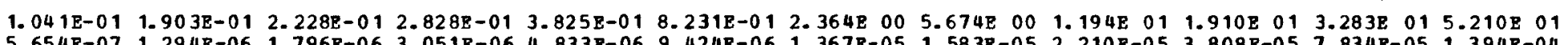

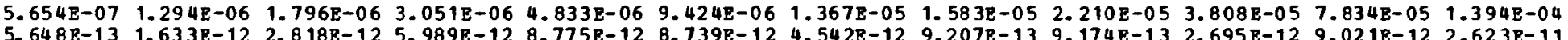

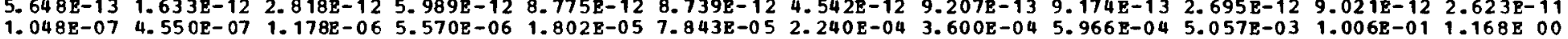
4.906E-11 1.192E-10 1.936E-10 3.976E-10 6.070E-10 9.784E-10 6.551E-09 1.451E-07 6.981E-06 2.583E-04 1.045E-02 2.088E-01

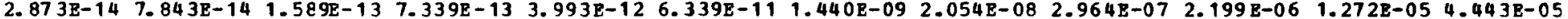

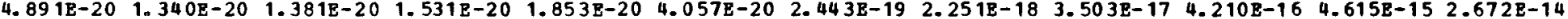

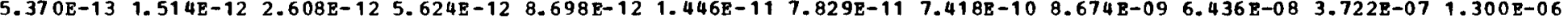

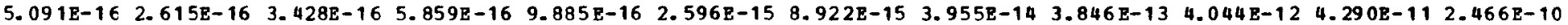
8.790E-09 1.941E-08 3.259E-08 8.198E-08 1.917E-07 1.097E-06 1. 1608-05 1.154E-04 1.351E-03 1.002E-02 5.796E-02 2.025E-01

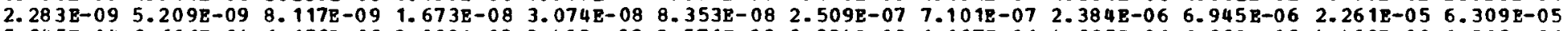
$5.845 \mathrm{E}-04$ 8.66 1E-04 $1.150 \mathrm{E}-03$ 2.008E-03 3.463E-03 9.571E-03 3.394E-02 1.167E-0 $4.325 \mathrm{E}-01$ 1.339E $00 \quad 4.460 \mathrm{E} \quad 00 \quad 1.263 \mathrm{E} 01$ $1.501 \mathrm{E}-04$ 1.542E-04 $1.578 \mathrm{E}-04$ 1.683E-04 1.859E-04 2.565E-04 5.042E-04 1.211E-03 3.671E-03 1.064E-02 $3.444 \mathrm{E}-02$ 9.546E-02

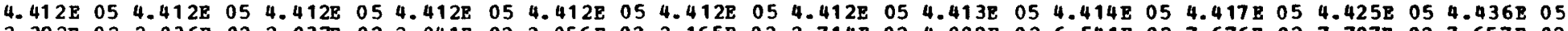

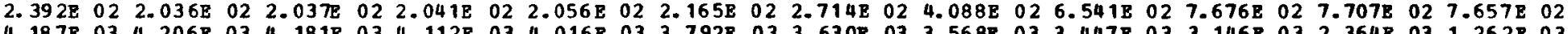

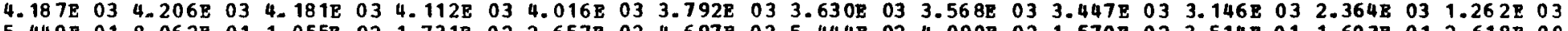

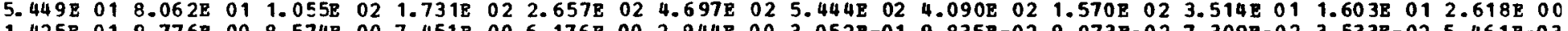
1. $425 \mathrm{E}$ 01 9.776E 00 8.574E 00 7.451E $006.176 \mathrm{E}$ 00 2.944E 00 3.052E-01 9.835E-02 9.073E-02 7.309E-02 $3.533 \mathrm{E}-025.461 \mathrm{E}-03$

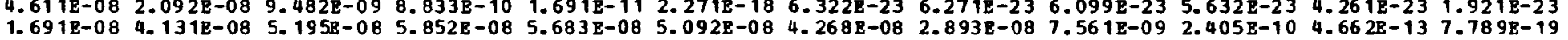

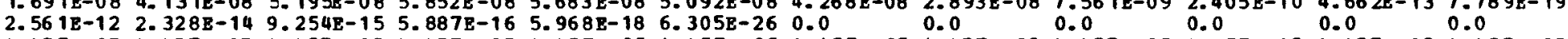

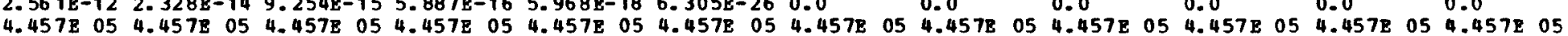


Table 2.22. Curies of light-element isotopes in a PWR assembly

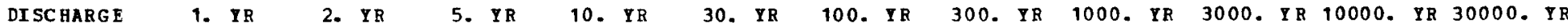

$\begin{array}{lr}H & 3 \\ \text { C } 14\end{array}$

CL 36

HN 54

FE 55

$\begin{array}{ll}\text { CO } 5 & 58 \\ \text { CO } & \end{array}$

Co 60

NI 59

NI 59
HI 63

ZN 65

Z 93

2R 95

NB 93U

IB 94

NB 95

yo 93

TC 99

IN113:

SN113

S.119

SE125

SOrTom

TCTAI

1. $214 \mathrm{E} 02 \quad 1.148 \mathrm{E} \quad 02 \quad 1.085 \mathrm{E} \quad 02 \quad 9.161 \mathrm{E} \quad 01 \quad 6.911 \mathrm{E} 012.239 \mathrm{E}$ 01 $4.334 \mathrm{E}-01 \quad 5.522 \mathrm{E}-06 \quad 4.077 \mathrm{E}-23 \quad 0.0$

0.0 0.0 $\begin{array}{lllllllll} & \end{array}$ . $126 \mathrm{E}-03$ 5.126E-03 $5.126 \mathrm{E}-03 \quad 5.126 \mathrm{E}-03 \quad 5.126 \mathrm{E}-03 \quad 5.126 \mathrm{E}-03 \quad 5.125$

.532E $011.965 \mathrm{E} 018.522 \mathrm{E} 006.948 \mathrm{E}-01 \quad 1.066 \mathrm{~B}-02 \quad 5.889 \mathrm{E}-100.0 \quad 0.0$

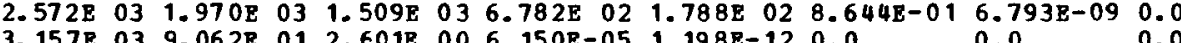

0.0

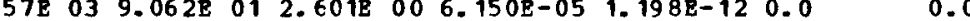

0.0
0.0
0.0

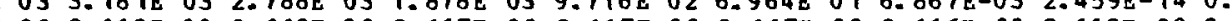

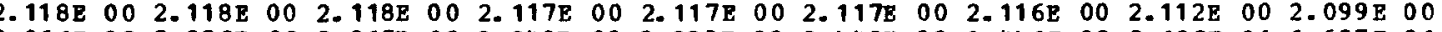

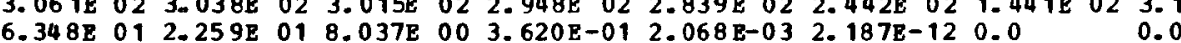

$1.049 \mathrm{E}-01$ 1.049E-01 $1.049 \mathrm{E}-01$ 1. $049 \mathrm{E}-01$ 1.049E-01 $1.049 \mathrm{E}-011.04$

$\begin{array}{llllllll}2.142 \mathrm{E} 04 & 4.489 \mathrm{E} 02 & 9.406 \mathrm{E} 00 & 8.656 \mathrm{E}-05 & 3.498 \mathrm{E}-13 & 0.0 & 0.0 & 0.0\end{array}$ 0

$6.576 \mathrm{E}-03 \quad 1.181 \mathrm{E}-02 \quad 1.674 \mathrm{E}-02 \quad 2.995 \mathrm{E}-02 \quad 4.746 \mathrm{E}-02 \quad 8.325 \mathrm{E}-02 \quad 9.940 \mathrm{E}-02 \quad 9.967 \mathrm{E}-02$

$6.242 \mathrm{E}-016.242 \mathrm{E}-016.242 \mathrm{E}-016.241 \mathrm{E}-016.240 \mathrm{E}-016.236 \mathrm{E}-016.220 \mathrm{E}-016.17$

0.0

$0.073 \mathrm{E}-08$
0.00 . $092 \mathrm{E}-035 \mathrm{5}-0$

$013 \mathrm{E}-034.79$

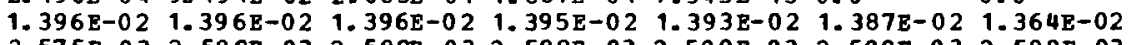

0
0

0.00101000

0.00 .0

$\begin{array}{lllllllll}575 \mathrm{E}-03 & 2.589 \mathrm{E}-03 & 2.589 \mathrm{E}-03 & 2.589 \mathrm{E}-03 & 2.589 \mathrm{E}-03 & 2.588 \mathrm{E}-03 & 2.588 \mathrm{E}-03 & 2.586 \mathrm{E}-03\end{array}$

$.029 \mathrm{E}-01$

$0.02 \mathrm{E}-01100$

0.0

126 02 4. $568 \mathrm{E}$ 01 $5.054 \mathrm{E} 006.85 \mathrm{E}-03 \mathrm{1}$

4.

$108 \mathrm{E}-02$

(1)

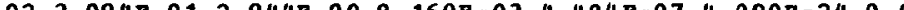

0.0

$.580 \mathrm{~B}-03.981 \mathrm{~B}-03$

000

$2.205 \mathrm{E}-0$

0.0

(1.3628-05

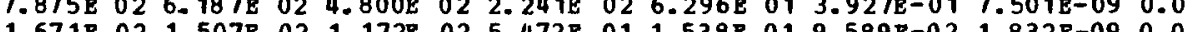

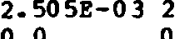

0.0

西

0.0
0.0
0.0

0.0
0.0

0.0

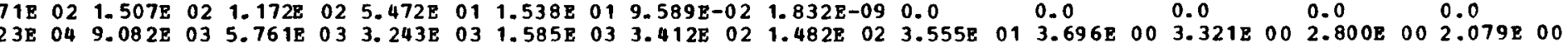

$\begin{array}{lll}0.0 & 0.0 \\ 0.0 & 0.0 & 0\end{array}$

$\begin{array}{ccc}0.0 & 0.0 & 0.0 \\ 0.0 & 0.0 & 0.0\end{array}$

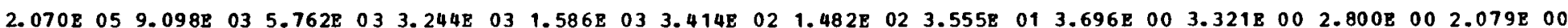


Table 2.23. Curies of fission-product isotopes in a PWR assembly

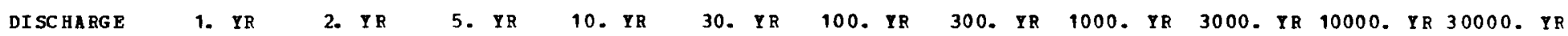

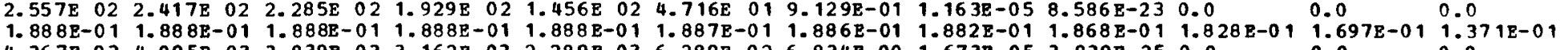

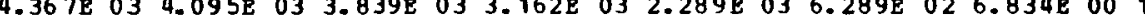
$\begin{array}{lllllllll}3.641 \mathrm{E} & 05 & 2.799 \mathrm{E} & 03 & 2.149 \mathrm{E} & 01 & 9.756 \mathrm{E}-06 & 2.615 \mathrm{E}-16 & 0.0\end{array}$ 0.0 0.0 $\begin{array}{llllll}0.0 & 0.0 & 0.0 & 0.0\end{array}$ 0.0

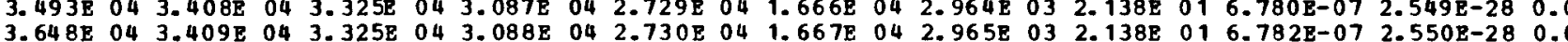
$4.784 \mathrm{E} 05$ 6.403E $03 \quad 8.511 \mathrm{E} 012.000 \mathrm{E}-04 \quad 8.308 \mathrm{E}-140.0$ 0.0 9.427E-02 1.606E-01 2.232E-01 3.908E-01 6.128E-01 1.067E 00 1.272E 00 1.275E 00 1.275E 00 1.273E 00 1.266E $001.248 \mathrm{E} \quad 00$

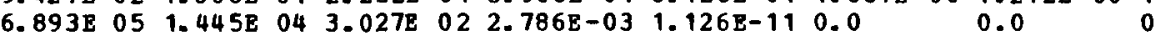

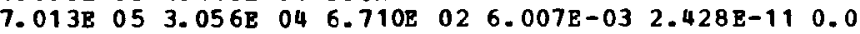
0.0

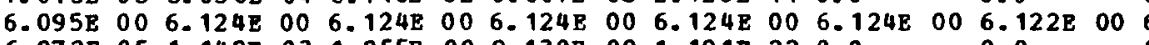
6. $872 \mathrm{E} 05$ 1.149E 03 1.955E 00 9.130R-09 1.191E-22 0.0

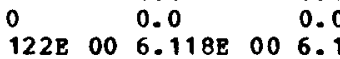
(1) 0.0 0.0 $2.87 \mathrm{E}$ 1. 150E 03 1.95 $2.57 \mathrm{E}$ 05 1.308E 05 6.584E 04 8.405E 03 2.720E 02 2.985E-04 $4.130 \mathrm{E}-250.0$

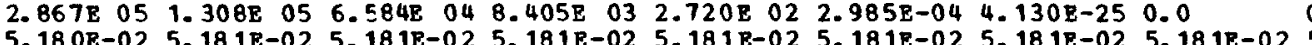

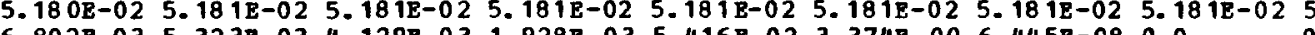

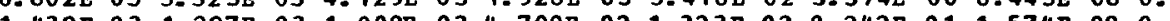

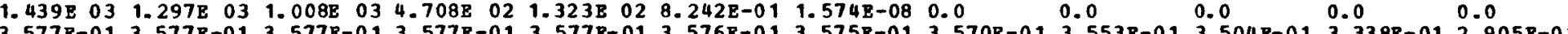
(1) 2.

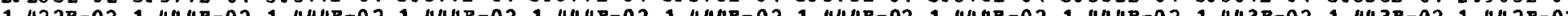

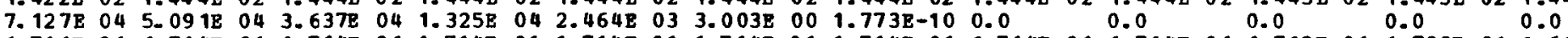
$1.711 \mathrm{E}-01$ 1.714E-01 1.714E-01 1.714E-01 1.714E-01 1.714E-01 1.714E-01 1.714E-01 1.714E-01 1.713E-01 1.709E-01 1.699E-01

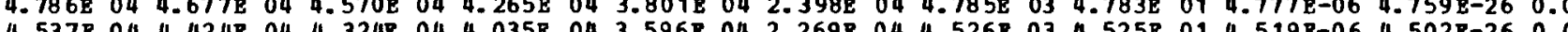

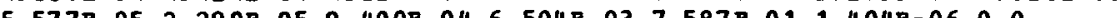

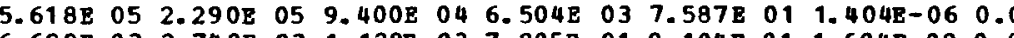
0.0

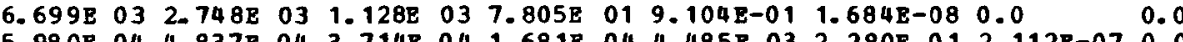

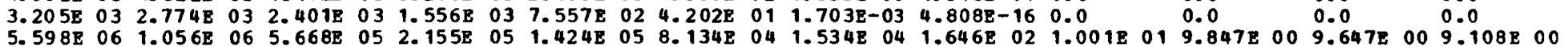


Table 2.24. Curies of heavy-element isotopes in a PWR assembly

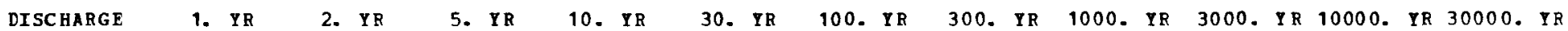

PE210

PB214

BI210

BI 214
PO2 10

$P 0214$

P02 14
P02 18

P02 18
RH 222

$R A 222$
$R \wedge 226$

$R \wedge 226$
$T$ H 230

TH234

T:233
P 2234

PA234
0234

0236

0238
$\times F 237$

บ 239

P 0238

P0239

P 0240

P 0241

PO242

A 1241

A 243

Ca242

CH244
S UATOT

TCTAL

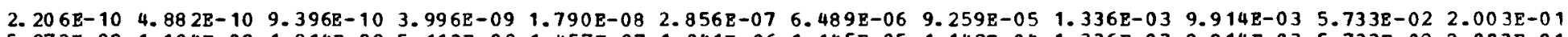
$5.873 \mathrm{E}-09$ 1.104E-08 $1.814 \mathrm{E}-08$ 5.113E-08 $1.457 \mathrm{E}-07$ 1.041E-06 $1.145 \mathrm{E}-05$ 1.142E-04 $1.336 \mathrm{E}-03$ 9.914E-03 $5.733 \mathrm{E}-02 \quad 2.003 \mathrm{E}-01$

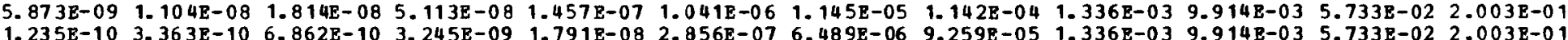

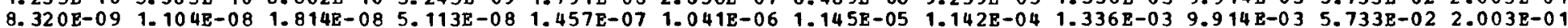
5.873E-09 1. $104 \mathrm{E}-08$ 1.814E-08 $5.113 \mathrm{E}-08$ 1.457E-07 $1.041 \mathrm{E}-06 \quad 1.145 \mathrm{E}-05$ 1.142E-04 1.336E-03 9.914E-03 $5.733 \mathrm{E}-02 \quad 2.003 \mathrm{E}-01$ 5.873E-09 1.104E-08 1.814E-08 5.113E-08 1.457E-07 1.041E-06 1.145E-05 1.142E-04 1.336E-03 9.914E-03 5.733E-02 2.003E-01

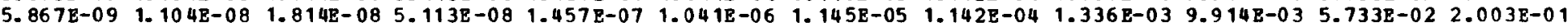
9.717E-06 1.417E-05 1.865E-05 $3.224 E-05 \quad 5.538 E-05 \quad 1.538 E-04 \quad 5.564 E-04 \quad 1.960 E-03 \quad 7.358 E-03 \quad 2.267 E-02 \quad 7.368 E-02 \quad 1.990 E-01$ $1.453 \mathrm{E}-01$ 1.451E-01 1.452E-0 1 1.451E-01 1.451E-01 1.451E-01 1.451E-01 1.451E-01 1.451E-01 1.451E-01 1.451E-01 1.451E-01 $1.348 \mathrm{E}-011.436 \mathrm{E}-01$ 1. $436 \mathrm{E}-01$ 1.440E-01 1.450E-01 1.5268-01 1.914E-01 2.883E-01 4.613E-01 5.413E-01 5.435E-01 5-400E-01 1.472E-01 1.451E-01 1.452E-01 1.451E-01 1.451E-01 1.451E-01 1.451E-01 1.451E-01 1.451E-01 1.451E-01 1.451E-01 1.451E-01 5.125E-01 5.154E-01 5.183E-01 5.272E-01 5.415E-01 5.935E-01 7.231E-01 8.643E-01 9.008E-01 8.968 E-01 8.821E-018.419 E-01 1. $159 \mathrm{E}-01$ 1.15 9E-01 1.159E-01 1.160E-01 1.160E-01 1. 161E-01 1.166E-01 1.180E-01 1.225E-01 1.337E-01 1.590E-01 1.799E-01 1.451E-01 1.451E-01 1.451E-01 1.451E-01 1.451E-01 1.451E-01 1.451E-01 1.451E-01 1.451E-01 1.451E-01 1.451E-01 1.451E-01 1. $403 \mathrm{E}-01$ 1. $436 \mathrm{E}-01$ 1.436E-0 1 $1.440 \mathrm{E}-01$ 1.450E-01 1.526E-01 1.914E-01 2.883E-01 4.613E-01 5.413E-01 5.435E-01 5.400E-01

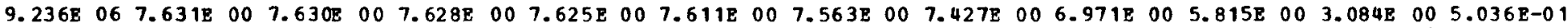
$9.832 \mathrm{E} 02$ 1.054E 03 1.06 1E 03 1.040E 03 1.001E 03 8.564E 02 4.970E 02 1.052E 02 4.867E-01 4.561E-06 6.144E-20 0.0

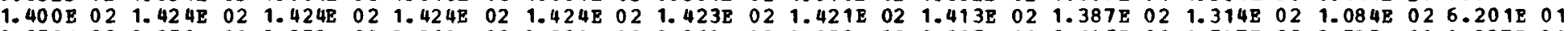

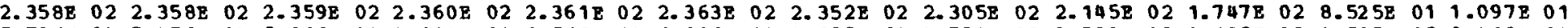
$5.736 \mathrm{E}$ 04 5.47 OE 04 5.216E 04 4.524E 04 3.568E 04 1.380E 04 4.975E 02 4.758E-02 9.583E-03 8. 103E-03 4.505E-03 8.419E-04 8. $294 \mathrm{E}-01$ 8. 295E-01 8.295E-01 8.295E-01 8.295E-01 8.294E-01 8.294E-01 8.291E-01 8.281E-01 8.251E-01 8.147E-01 7.855E-01

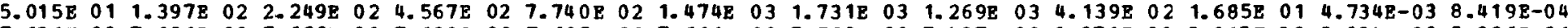

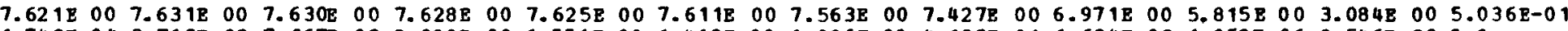

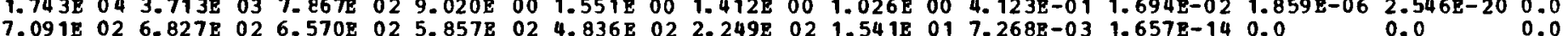

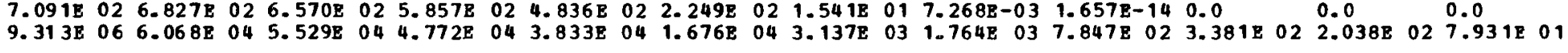


Table 2.25. Watts of light-element isotopes in a PWR assembly

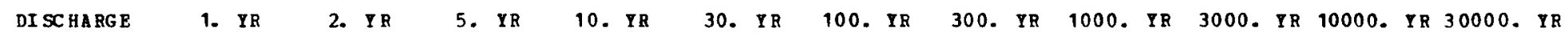
$\begin{array}{llllllllllll}6.341 \mathrm{E}-04 & 6.340 \mathrm{O}-04 & 6.339 \mathrm{E}-04 & 6.337 \mathrm{E}-04 & 6.333 \mathrm{E}-04 & 6.318 \mathrm{E}-04 & 6.265 \mathrm{E}-04 & 6.115 \mathrm{E}-04 & 5.6\end{array}$

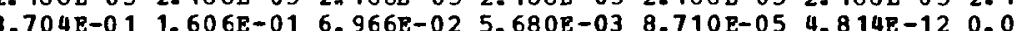
(1. 4. $321 \mathrm{E} 01$ 1.240E 00 3. $560 \mathrm{E}-02$ 8. $418 \mathrm{E}-07$ 1.640E-14 0.0 0.0
0.0
0.0 $148 \mathrm{E}-04 \quad 4.109$

1. $541 \mathrm{E} 00 \quad 1.705 \mathrm{E}-01$ 1.886-02 $2.487 \mathrm{E}-05 \quad 4.122 \mathrm{E}-10 \quad 3.113 \mathrm{E}-29 \quad 0.0$

0.0 $0.0 \quad 0.70$

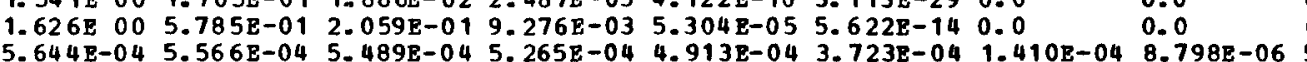

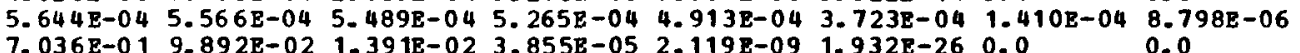
$\begin{array}{lllllll}7.036 \mathrm{E}-01 & 9.892 \mathrm{E}-02 & 1.391 \mathrm{E}-02 & 3.855 \mathrm{E}-05 & 2.119 \mathrm{E}-09 & 1.932 \mathrm{E}-26 & 0.0\end{array}$

0.0
0.0

$0.759 \mathrm{E}-051.70$

$2.516 \mathrm{E}$ O0 1.977E 00 1.534E 00 7.160B-01 2.012E-01 1.255E-03 2.397E-11 0.0

0.0

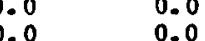

1. $426 \mathrm{E}-01$ 1. $287 \mathrm{E}-01$ 1.000E-01 $4.671 \mathrm{E}-02$ 1.312E-02 8.185E-05 $1.564 \mathrm{E}-12 \quad 0.0$ $5.341 \mathrm{E}-10$

0.0

0.0

0 0. 0 $\begin{array}{ll}.891 \mathrm{E}-04 & 1.683 \mathrm{E}-05 \\ .139 \mathrm{E}-05 & 2.046 \mathrm{E}-05\end{array}$

TOTAL 


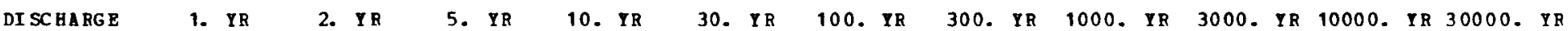

$\begin{array}{llllllllllll}4.700 \mathrm{E}-05 & 4.700 \mathrm{E}-05 & 4.700 \mathrm{E}-05 & 4.700 \mathrm{E}-05 & 4.699 \mathrm{E}-05 & 4.698 \mathrm{E}-05 & 4.695 \mathrm{E}-05 & 4.685 \mathrm{E}-05 & 4.650 \mathrm{E}-05 & 4.552 \mathrm{E}-05 & 4.224 \mathrm{E}-05 & 3.412 \mathrm{E}-05\end{array}$

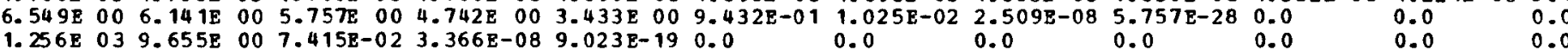
4.099E 01 3.999E $013.902 \mathrm{E} 013.624 \mathrm{E} 013.203 \mathrm{E} 01$ 1.956E 01

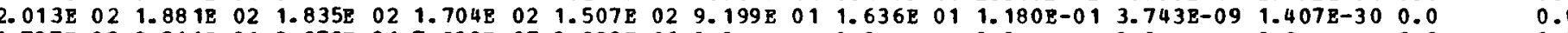

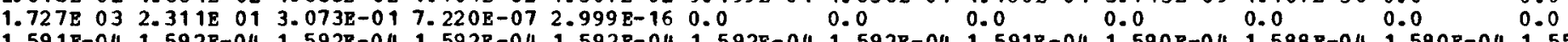
$1.591 \mathrm{E}-04$ 1.592E-04 1.592E-04 1.592E-04 1.592E-04 1.592E-04 1.592E-04 1.591E-04 1.590E-04 1.588E-04 $1.580 \mathrm{E}-04 \quad 1.557 \mathrm{E}-04$

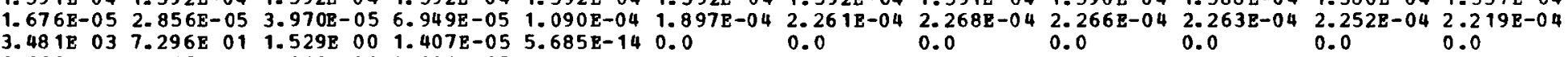

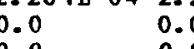
0.0 3. $363 \mathrm{E} 031.465 \mathrm{E} 02 \quad 3.218 \mathrm{E}$ 00 $2.881 \mathrm{E}-05$ 1. $164 \mathrm{E}-130.0$ 0.0 0.000 .0

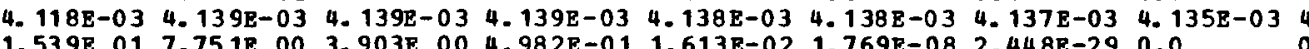
$1.539 \mathrm{E} 01$ 7.751E 00 3.903E $00 \quad 4.982 \mathrm{E}-01$ 1.613E-02 $1.769 \mathrm{E}-08 \quad 2.448 \mathrm{E}-290.0$ $2.796 \mathrm{E} 03$ 1.275E $03 \quad 6.420 \mathrm{E} 028.196 \mathrm{E} 01 \quad 2.653 \mathrm{E} 00 \quad 2.911 \mathrm{E}-06 \quad 4.027 \mathrm{E}-27 \quad 0.0$ $3.331 \mathrm{E} 011.219 \mathrm{E} 014.465 \mathrm{E} 002.191 \mathrm{E}-01$ 1.445E-03 $2.707 \mathrm{E}-12 \quad 0.0$ .173E 01 1.701E 01 1.319E 01 6.159E 00 1.731E 00 1.078E-02 2.059E-10 0.0 $\begin{array}{llll}0.0 & 0.0 & 0.0 & 0.0 \\ 4.125 \mathrm{E}-03 & 4.098 \mathrm{E}-03 & 4.005 \mathrm{E}-03 & 3.752 \mathrm{E}-03\end{array}$ 0.000 .0038

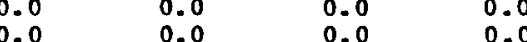
. (1)

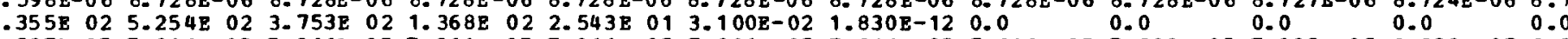

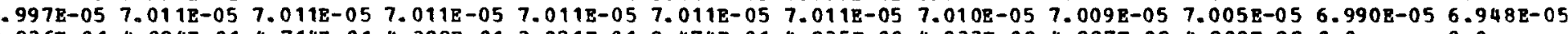
$\begin{array}{lllllllllll} & \end{array}$

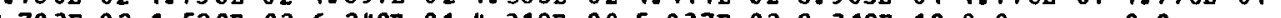
. 0.0 4. $309 \mathrm{E} 031.756 \mathrm{E} 03 \quad 7.210 \mathrm{E} 02 \quad 4.989 \mathrm{E}$ 01 $5.819 \mathrm{E}-01$ 1.077E-08 0.0 0.0 $-08 \quad 1.766 \mathrm{~B}-28 \quad 0.0$ $\begin{array}{llll}0 & 0.0 & 0.0 & 0.0 \\ 230 \mathrm{E}-03 & 1.205 \mathrm{E}-05 & 4.010 \mathrm{E}-12 & 0.0\end{array}$

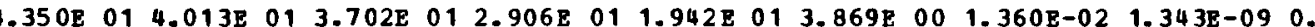
0.0 $\begin{array}{lllllllll} & \end{array}$ $\begin{array}{ll}0.0 & 0.0\end{array}$ 0.0 
Table 2.27. Watts of heavy-element isotopes in a PWR assemb1y

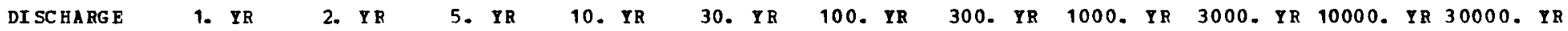

BI214

PC210

P0214

P0218

RN222

TH230

0234

0236

0238

N 2237

P 0238

PU239

P 0240

P 0241

PU242

A. 241

An243

CH242

CM243

CH244

moTal

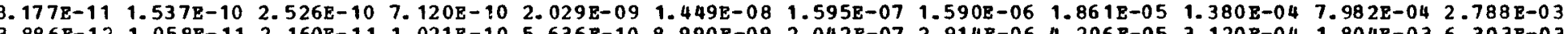

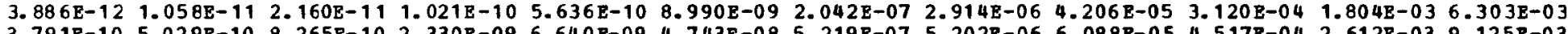
3.791E-10 5.029E-10 8.265E-10 2.330E-09 6.640E-09 4.743E-08 5.219E-07 5.202E-06 6.088E-05 4.517E-04 2.612E-03 9.125E-03

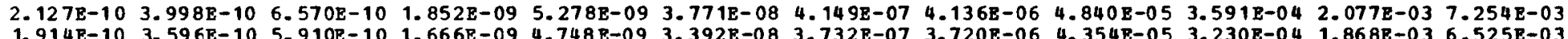
1. $914 \mathrm{E}-10$ 3. 596E-10 5.910E-10 1.666E-09 4.748E-09 3.392E-08 3.732E-07 3.720E-06 $4.354 \mathrm{E}-05$ 3.230E-04 1.868E-03 $6.525 \mathrm{E}-03$

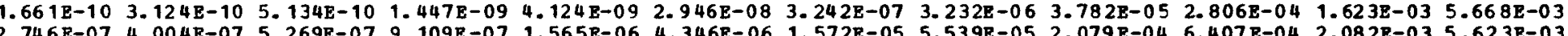
2.746E-07 4.004E-07 5.269E-07 9. 109E-07 1.565E-06 $4.346 \mathrm{E}-06 \quad 1.572 \mathrm{E}-05$ 5.539E-05 2.079E-04 6.407 E-04 $2.082 \mathrm{E}-03 \quad 5.623 \mathrm{E}-03$ 1. $475 \mathrm{E}-02$ 1.48 3E-02 1.492E-02 1.517E-02 1.55 9E-02 1.708E-02 2.08 1E-02 2.488E-02 2.5938-02 2.581B-02 2.539E-02 2.423E-02 $3.143 \mathrm{E}-03$ 3.14 3E-03 3.143E-03 3.143E-03 3.144E-03 3.148E-03 3.161E-03 3.198E-03 3.320E-03 3.625E-03 4.3108-03 $4.877 \mathrm{E}-03$ $3.672 E-03 \quad 3.672 E-03 \quad 3.672 E-03 \quad 3.672 E-03$ 3.672E-03 3.672E-03 3.672E-03 3.672E-03 3.672E-03 3.672E-03 $3.6728-03$ 3.672E-03 4. $121 \mathrm{E}-03$ 4. $218 \mathrm{E}-03$ 4. $419 \mathrm{E}-03$ 4.229E-03 $4.259 \mathrm{E}-03$ 4. $484 \mathrm{E}-03$ 5.623E-03 $8.469 \mathrm{E}-03$ 1.355E-02 $1.590 \mathrm{E}-02 \quad 1.597 \mathrm{E}-02 \quad 1.586 \mathrm{E}-02$ $3.256 \mathrm{E} 013.492 \mathrm{E} 013.513 \mathrm{E} 01$ 3.445E 01 3.314E 01 2.836E 01 1.646E 01 3.483E $001.612 \mathrm{E}-021.510 \mathrm{E}-072.035 \mathrm{E}-210.0$

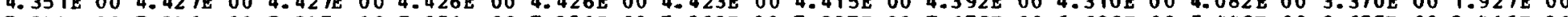
$7.344 \mathrm{E} 00$ 7.346E 00 7.347E $007.351 \mathrm{E}$ 00 7.356E 00 7.362E 00 7.327E 00 7.179E 00 6.682E 00 5.443E 00 2.655E 00 3.4168-01 2.38 OE 00 2.27 0E $002.164 \mathrm{E}$ 00 $1.877 \mathrm{E}$ 00 1. $480 \mathrm{E}$ 00 $5.728 \mathrm{E}-01$ 2.064E-02 1.974E-06 3.976E-07 3.362 E-07 1.869E-07 3.493 E-08

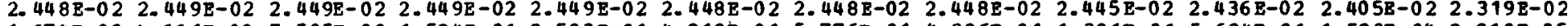

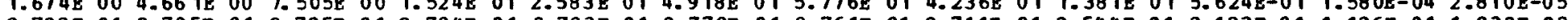
2.782E-01 2.785E-01 2.785E-01 2.784E-01 2.78JE-01 2.778E-01 2.761E-01 2.711E-01 2.544E-01 2.123E-01 1.126E-01 1.838B-02

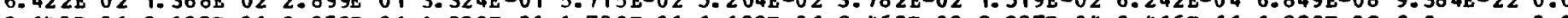
$2.1498-01$ 2.103E-01 2.058E-01 1.928E-01

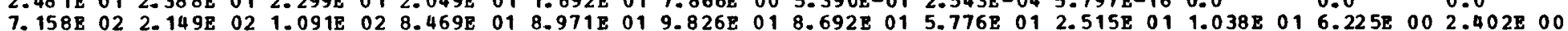

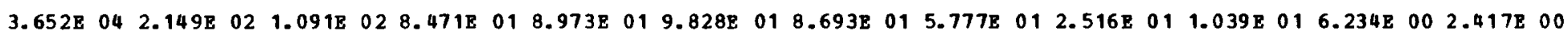


12 GROUP PHOTON RELEASE RATES, PHOTONS/S BCOND

EMEAN

2. $\mathrm{YR}$

5. TR TIME APTER DISCHARGE

100. IR 300. YR 1000. TR 3000. YR 10000. YR 30000. YR

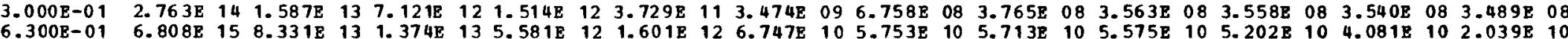

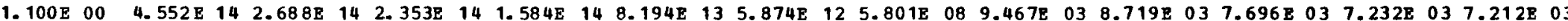

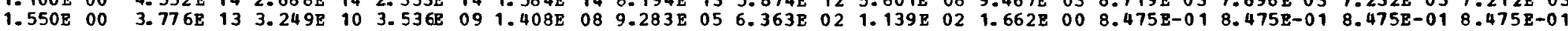

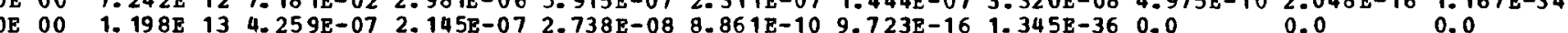

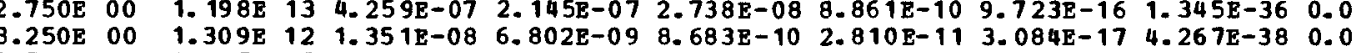
(1) 6.715 0.0
0.0 4. $175 \mathrm{E} .110 .0$

TOTAL 0.0
0.0 0.0
0.0 0.0
0.0
0.0 0.0
0.0
0.0
0.0
0.0
0.0 0.0
0.0 0.0 0.0
0.0
0.0

$\mathrm{HV} / \mathrm{S} \mathrm{EC}$

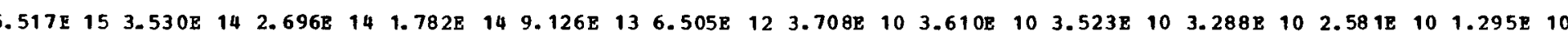
12 GROUP SPECI PIC ENERGY RELEASE RATES, HEV/RATT-SEC

EEEAN

DISCHABGE
1. $\mathrm{YR}$
2. $Y R$

5. $I R^{2}$

10. YR

30. YR

100. IR

300. $\mathrm{Y}$

1000. IR 3000. IR 10000. IR 30000. YR

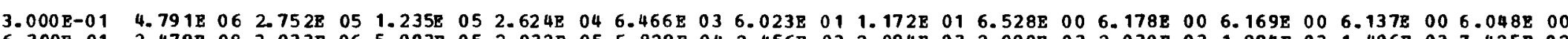

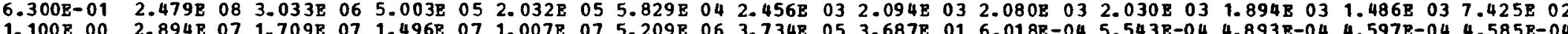

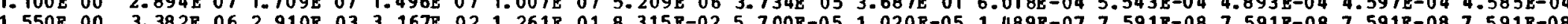

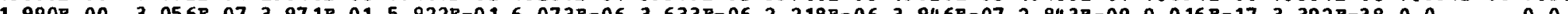

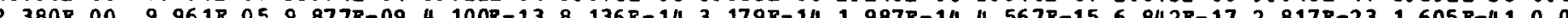

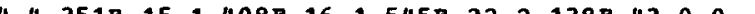

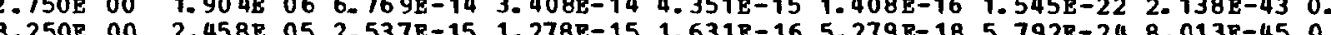
$3.700 \mathrm{E} 00 \quad 8.820 \mathrm{E} 000.0 \quad 0.0 \quad 0.0 \quad 0.0 \quad 0.00000$ $4.220 \mathrm{E} 00$ 
Table 2.29. Photon spectrum for fission-product isotopes in a PWR assembly

12 GROUP PHOTON RELEASE RATES, PHOTONS/SECOND

EHEAN

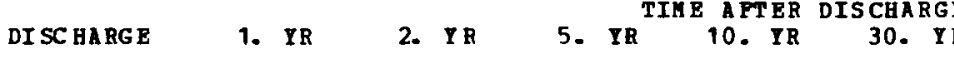

30. YR 100. IR 300. IR 1000. IR 3000. IR 10000. YR 30000. IR

$3.000 \mathrm{E}-01$

6. $300 \mathrm{E}-01$

$\begin{array}{ll}1.100 \mathrm{E} & 00 \\ 1.550 \mathrm{E} & 00\end{array}$

$\begin{array}{ll}1.550 \mathrm{E} & 00 \\ 1.990 \mathrm{E} & 00\end{array}$

$2.380 \mathrm{E}$ OO

$\begin{array}{lll}2.750 \mathrm{E} & 00 \\ 3.250 \mathrm{E} & 00\end{array}$

$3.7 \mathrm{COE} 00$

4.220800

$4.700 \mathrm{E} 00$

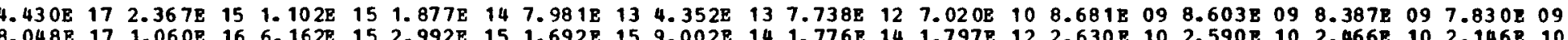

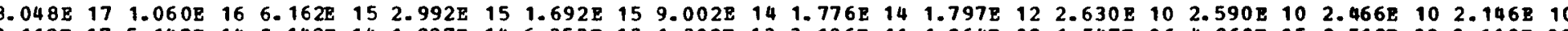

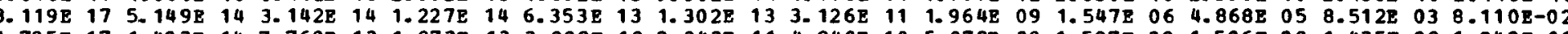

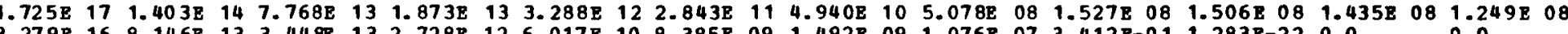
$\begin{array}{llllllll} & \end{array}$

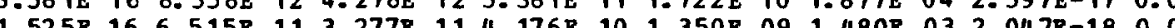

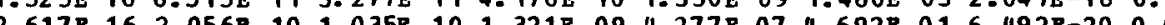

. $1463 \mathrm{~B} 150.0$

4.

0.0

0.0

0.0

0.0

0.0

0.0

0.0

5.250800

$0.0 \quad 0.0 \quad 0.0$

$0.0 \quad 0.0$

0.0
0.0
0.0

0.0

$0.0 \quad 0.0$

$0.0 \quad 0.0$

$\begin{array}{lll}0.0 & 0.0 & 0.0\end{array}$

TOTAL

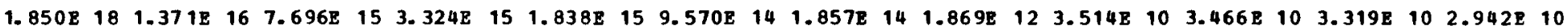

HET/SEC

$\begin{array}{llllllllllllllllllllllllll}1.562 \mathrm{E} & 18 & 8.354 \mathrm{E} & 15 & 4.759 \mathrm{E} & 15 & 2.112 \mathrm{E} & 15 & 1.165 \mathrm{E} & 15 & 5.949 \mathrm{E} & 14 & 1.147 \mathrm{E} & 14 & 1.156 \mathrm{E} & 12 & 1.941 \mathrm{E} & 10 & 1.913 \mathrm{E} & 10 \quad 1.827 \mathrm{E} & 10 \quad 1.606 \mathrm{E} & 10\end{array}$

12 GROOP SPECI FIC BNRRGY RELEASE RATES, HEV/RATT-SEC

EHEAH

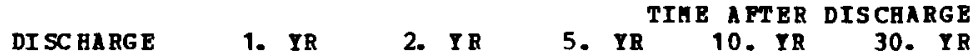

100. YR 300. IR 1000. IR 3000. YR 10000. IR 30000. TR

$3.000 \mathrm{E}-01$ $6.300 \mathrm{E}-01$

$1.100 \mathrm{E} 00$

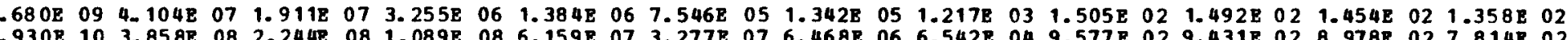
$\begin{array}{llllllllllllllll} & \end{array}$

1.5508 00

$2.380 \mathrm{E} 00$

$2.750 \mathrm{E} 00$

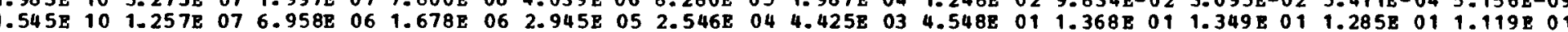

$3.250 \mathrm{E}$ 00 (1) $4.926 \mathrm{E}$ 09 1.177E 06 5.884E 05 7.401E $04 \quad 2.368 \mathrm{E}$ 03 $2.582 \mathrm{E}-03$ 3.572E-24 0.0

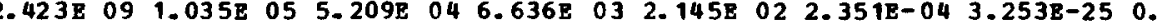

$\begin{array}{ll}3.700 E & 00 \\ 4.2208 & 00\end{array}$

$\begin{array}{ll}4.220 \mathrm{E} & 00 \\ 4.700 \mathrm{~B} & 00\end{array}$

$\begin{array}{ll}4.700 \mathrm{E} & 00 \\ 5.250 \mathrm{E} & 00\end{array}$ 3.861E 03 1.

$3.129 \mathrm{E} 080.0$

$1.006 \mathrm{E} 090.0$

080.0

$\begin{array}{llll}1.944 \mathrm{E} & 03 & 2.482 \mathrm{E} & 02 \\ 0.0 & 0.0 & 0.0\end{array}$

$.033 \mathrm{E} 00$

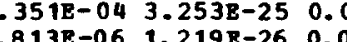

$.424 \mathrm{E} 080.0$

$0.0 \quad 0.0$

0.0

0.0

0.0

0.0

0.0
0.0
0.0

0.0
0.0

1.2
0.0
0.0

0.0

0.0

0.0

0.0

0.0

0.0
0.0

3.0
0.0
0.0

0.0
0.0
0.0
0.0

TOTAL

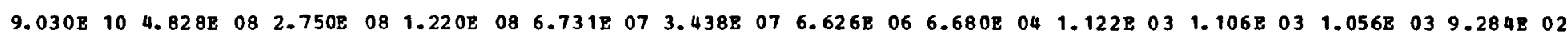

GAI POR

$\begin{array}{lllllllllllllllllllll}2.505 \mathrm{E} & 05 & 1.339 \mathrm{E} & 03 & 7.628 \mathrm{E} & 02 & 3.385 \mathrm{E} & 02 & 1.867 \mathrm{E} & 02 & 9.537 \mathrm{E} & 01 & 1.838 \mathrm{E} & 01 & 1.853 \mathrm{E}-01 & 3.112 \mathrm{E}-03 & 3.067 \mathrm{E}-03 & 2.929 \mathrm{E}-03 & 2.575 \mathrm{E}-03\end{array}$ 
$3.000 \mathrm{E}-0$ $6.300 \mathrm{E}-01$ $1.100 \mathrm{E} 00$ $1.550 \mathrm{E} 00$ $2.380 \mathrm{E} 00$ 2.750800 $3.250 \mathrm{E} 00$ $3.700 \mathrm{E} \mathrm{O}$ $4.220 \mathrm{E} 00$ $4.700 \mathrm{E} 00$ $5.250 \mathrm{E} 00$ $3.000 \mathrm{E}-0$ 4. $000 \mathrm{E}-0$ $6.000 \mathrm{E}-02$

TOTA L

GEV/SEC

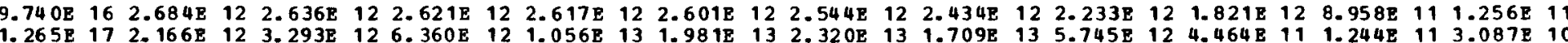
$1.940 \mathrm{E} 172.091 \mathrm{E} 10 \mathrm{1.781E} 10 \mathrm{G}$

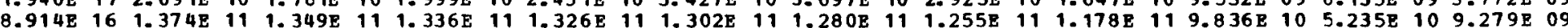

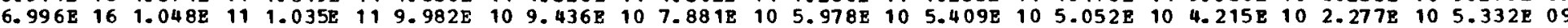

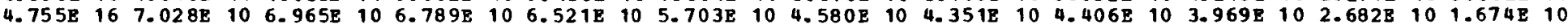

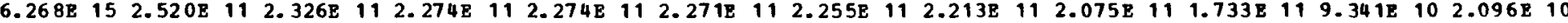

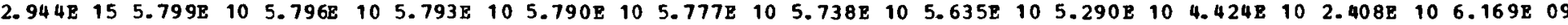

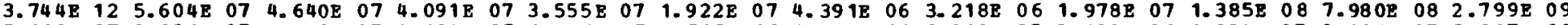

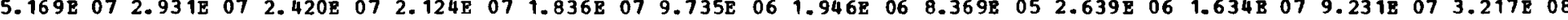

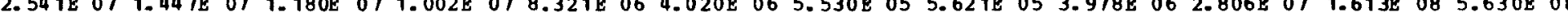

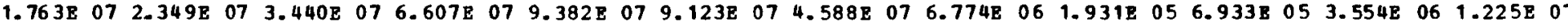

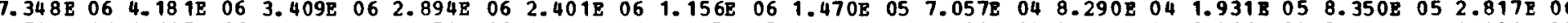

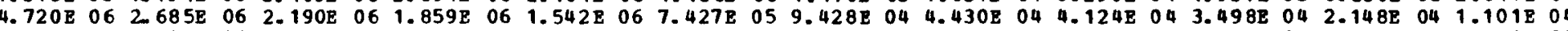

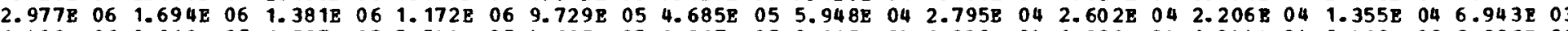

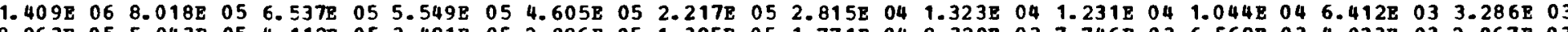

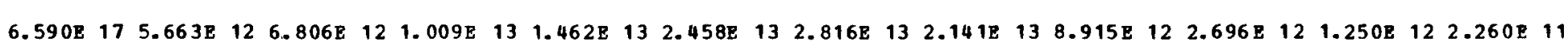

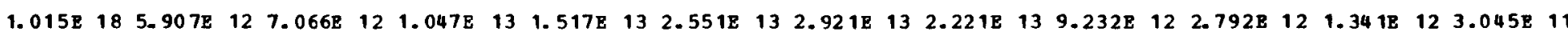

\section{GROUP SPECI PIC ENERGY RELEASE RATES, HEV/RATT-SEC}

BABAR

DISCHARG

1. $\mathrm{YR}$

2. $Y R$

5. $Y R^{\text {TIME }}$ APTER

(30.

100. $Y$

300. YR 1000. YR 3000. Y 10000 . YR 30000. TR

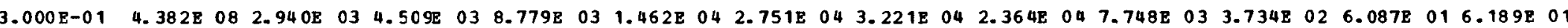

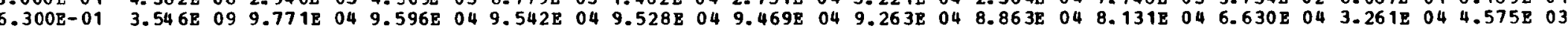

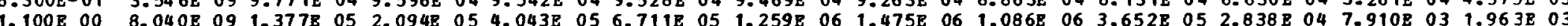

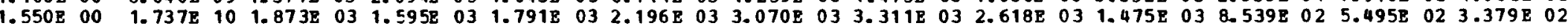

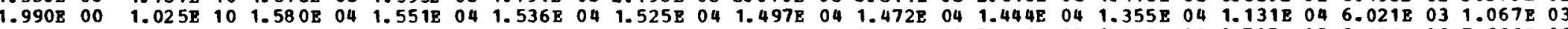

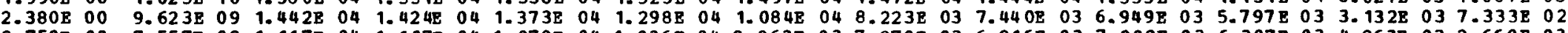

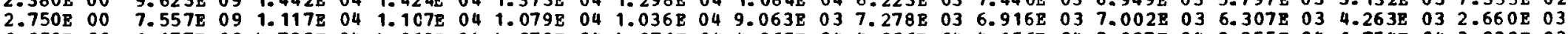

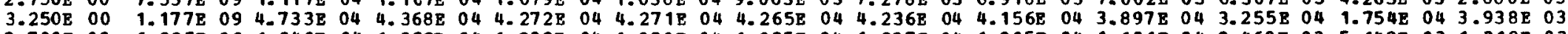

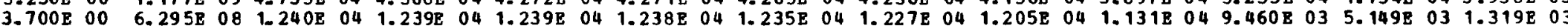

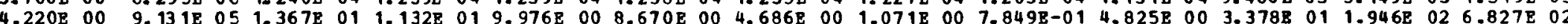

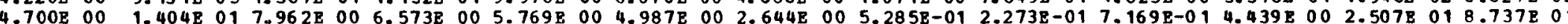

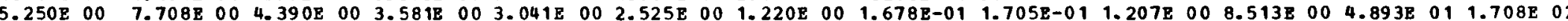
3.000E-02 3.057E-02 4.072E-02 5.964E-02 1.146E-01 1.627 E-01 1.582E-01 7.954E-02 $1.174 \mathrm{E}-02$ 3.347E-04 $1.202 \mathrm{E}-03 \quad 6.162 \mathrm{E}-03 \quad 2.124 \mathrm{E}-02$ $4.000 \mathrm{E}-02 \quad 1.699 \mathrm{E}-02 \quad 9.665 \mathrm{E}-03 \quad 7.881 \mathrm{E}-03 \quad 6.690 \mathrm{E}-03 \quad 5.551 \mathrm{E}-03 \quad 2.673 \mathrm{E}-03 \quad 3.397 \mathrm{E}-04 \quad 1.631 \mathrm{E}-04 \quad 1.916 \mathrm{E}-04 \quad 4.463 \mathrm{E}-04 \quad 1.930 \mathrm{E}-03 \quad 6.512 \mathrm{E}-03$ $\begin{array}{lllllllllllll}6.000 z-02 & 1.636 z-02 & 9.312 E-03 & 7.593 E-03 & 6.445 E-03 & 5.348 z-03 & 2.575 z-03 & 3.269 E-04 & 1.536 E-04 & 1.4308-04 & 1.213 E-04 & 7.447 z-05 & 3.816 E-05\end{array}$

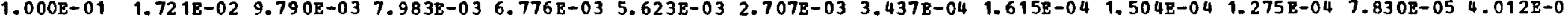
1.22 1E-02 6.950E-03 5.667E-03 $4.810 \mathrm{E}-03$ 3.992E-03 1.922E-03 $2.440 \mathrm{E}-04 \quad 1.147 \mathrm{E}-04 \quad 1.067 \mathrm{E}-04 \quad 9.052 \mathrm{E}-05 \quad 5.558 \mathrm{E}-05 \quad 2.848 \mathrm{E}-05$ $2.000 E-01$ 1. 02 4E-02 5.829E-03 4.753E-03 4.034E-03 3.348E-03 1.612E-03 2.047E-04 9.616E-05 8.953E-05 7:592E-05 4.662E-05 2.389E-05

TOTAI

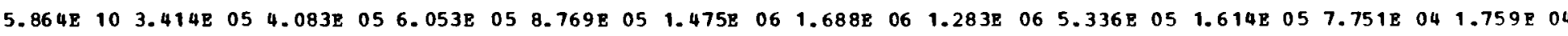

GAM PON 1.627E $059.468 \mathrm{E}-01$ 1.133E 00 1.679E $00 \quad 2.432 \mathrm{E} 00 \quad 4.090 \mathrm{E} 00 \quad 4.682 \mathrm{E} 00 \quad 3.560 \mathrm{E} \quad 00 \quad 1.480 \mathrm{E} \quad 004.476 \mathrm{E}-012.150 \mathrm{E}-014.880 \mathrm{E}-02$ 
Table 2.31. Total curies in PWR assembly as a function of age

\begin{tabular}{rlccc}
\hline $\begin{array}{c}\text { Years after } \\
\text { discharge }\end{array}$ & $\begin{array}{c}\text { Activation } \\
\text { products }\end{array}$ & $\begin{array}{c}\text { Heavy } \\
\text { elements }\end{array}$ & $\begin{array}{c}\text { Fission } \\
\text { products }\end{array}$ & Total \\
\hline 0 & $2.070 \times 10^{5}$ & $1.932 \times 10^{7}$ & $7.293 \times 10^{7}$ & $9.246 \times 10^{7}$ \\
1 & $9.098 \times 10^{3}$ & $6.069 \times 10^{4}$ & $1.059 \times 10^{6}$ & $1.129 \times 10^{6}$ \\
2 & $5.762 \times 10^{3}$ & $5.530 \times 10^{4}$ & $5.673 \times 10^{5}$ & $6.284 \times 10^{5}$ \\
5 & $3.244 \times 10^{3}$ & $4.773 \times 10^{4}$ & $2.155 \times 10^{5}$ & $2.665 \times 10^{5}$ \\
10 & $1.586 \times 10^{3}$ & $3.834 \times 10^{4}$ & $1.425 \times 10^{5}$ & $1.824 \times 10^{5}$ \\
30 & $3.414 \times 10^{2}$ & $1.676 \times 10^{4}$ & $8.135 \times 10^{4}$ & $9.845 \times 10^{4}$ \\
100 & $1.482 \times 10^{2}$ & $3.140 \times 10^{3}$ & $1.534 \times 10^{4}$ & $1.863 \times 10^{4}$ \\
300 & $3.555 \times 10^{2}$ & $1.765 \times 10^{3}$ & $1.646 \times 10^{2}$ & $1.965 \times 10^{3}$ \\
1,000 & 3.696 & $7.848 \times 10^{2}$ & $1.001 \times 10^{1}$ & $7.985 \times 10^{2}$ \\
3,000 & 3.321 & $3.381 \times 10^{2}$ & 9.847 & $3.513 \times 10^{2}$ \\
10,000 & 2.800 & $2.040 \times 10^{2}$ & 9.647 & $2.164 \times 10^{2}$ \\
30,000 & 2.079 & $7.978 \times 10^{1}$ & 9.108 & $9.097 \times 10^{1}$ \\
\hline
\end{tabular}

Table 2.32. Total watts of thermal power in PWR assembly as a function of age

\begin{tabular}{clccc}
\hline $\begin{array}{c}\text { Years after } \\
\text { discharge }\end{array}$ & $\begin{array}{c}\text { Activation } \\
\text { products }\end{array}$ & $\begin{array}{c}\text { Heavy } \\
\text { elements }\end{array}$ & $\begin{array}{c}\text { Fission } \\
\text { products }\end{array}$ & Total \\
\hline 0 & $1.378 \times 10^{3}$ & $3.652 \times 10^{4}$ & $8.702 \times 10^{5}$ & $9.081 \times 10^{5}$ \\
1 & $6.753 \times 10^{1}$ & $2.149 \times 10^{2}$ & $4.527 \times 10^{3}$ & $4.809 \times 10^{3}$ \\
2 & $5.102 \times 10^{1}$ & $1.091 \times 10^{2}$ & $2.327 \times 10^{3}$ & $2.487 \times 10^{3}$ \\
5 & $3.323 \times 10^{1}$ & $8.471 \times 10^{1}$ & $7.307 \times 10^{2}$ & $8.486 \times 10^{2}$ \\
10 & $1.683 \times 10^{1}$ & $8.973 \times 10^{1}$ & $4.188 \times 10^{2}$ & $5.254 \times 10^{2}$ \\
30 & 1.285 & $9.828 \times 10^{1}$ & $2.303 \times 10^{2}$ & $3.299 \times 10^{2}$ \\
100 & $7.816 \times 10^{-2}$ & $8.693 \times 10^{1}$ & $4.258 \times 10^{-1}$ & $1.296 \times 10^{2}$ \\
300 & $3.325 \times 10^{-2}$ & $5.777 \times 10^{1}$ & $3.822 \times 10^{-1}$ & $5.819 \times 10^{1}$ \\
1,000 & $2.033 \times 10^{-2}$ & $2.516 \times 10^{1}$ & $1.001 \times 10^{-2}$ & $2.519 \times 10^{1}$ \\
3,000 & $1.949 \times 10^{-2}$ & $1.039 \times 10^{1}$ & $9.894 \times 10^{-3}$ & $1.042 \times 10^{1}$ \\
10,000 & $1.719 \times 10^{-2}$ & 6.234 & $9.544 \times 10^{-3}$ & 6.261 \\
30,000 & $1.275 \times 10^{-2}$ & 2.417 & $8.624 \times 10^{-3}$ & 2.438 \\
\hline
\end{tabular}




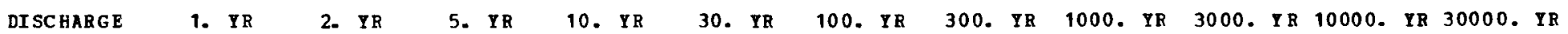

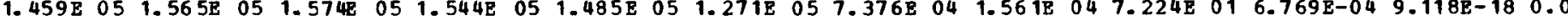

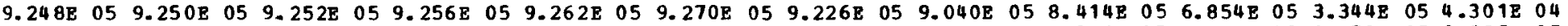

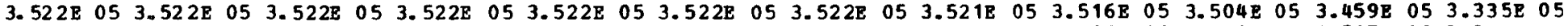

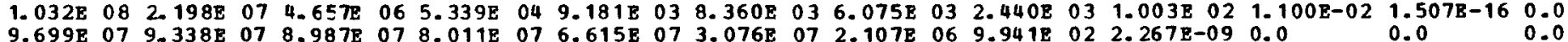

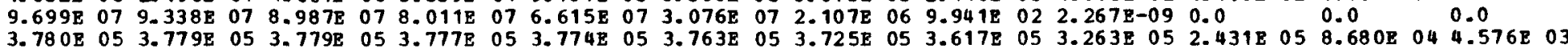

2.024E-07 5.512E-07 1.125E-06 5.318E-06 2.935E-05 4.682E-04 1.064E-02 1.518E-01 2.1908 00 1.625E 01 9.397E 01 3.28 2E 02

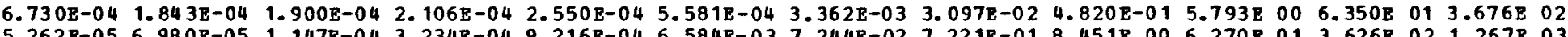
5.262E-05 6.98 OE-05 1.147E-04 3.234E-04 9.216E-04 6.584E-03 7.244E-02 7.221E-01 8.451E 00 6.270E 01 3.626E 02 1.267E 03

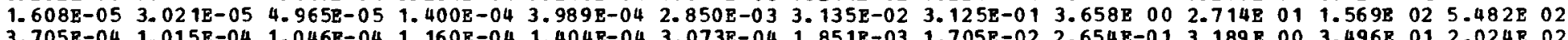

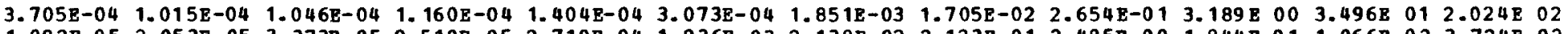

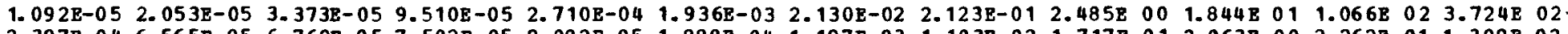
2. 397E-04 6.565E-05 6.769E-05 7.502E-05 9.082E-05 $1.988 \mathrm{E}-04 \quad 1.197 \mathrm{E}-03 \quad 1.103 \mathrm{E}-02 \quad 1.717 \mathrm{E}-01 \quad 2.063 \mathrm{E} \quad 00 \quad 2.262 \mathrm{E} \quad 01 \quad 1.309 \mathrm{~B} \quad 02$

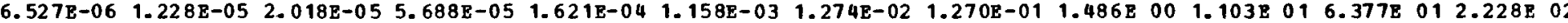

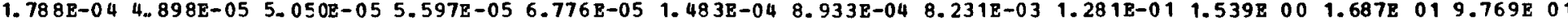

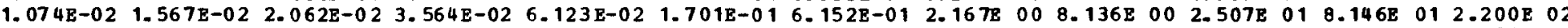

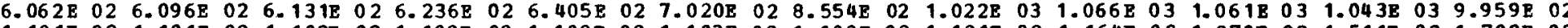

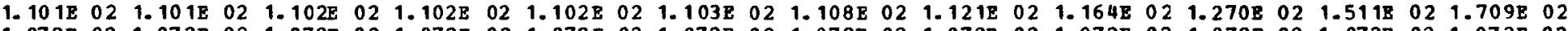

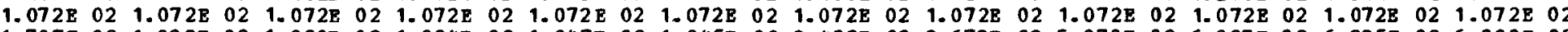

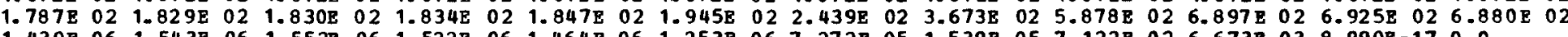

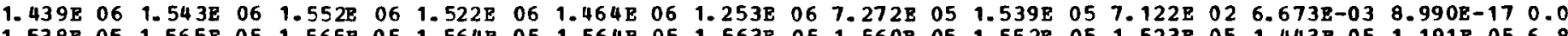

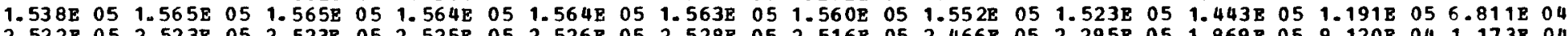

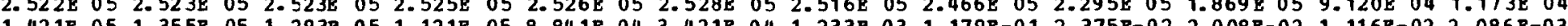

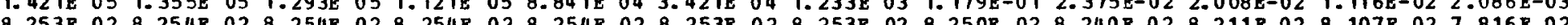

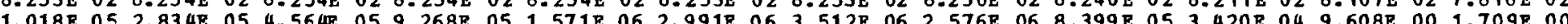
(1.

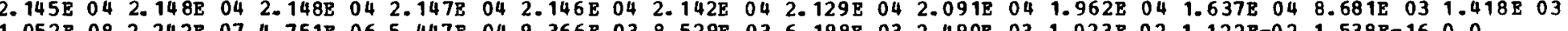

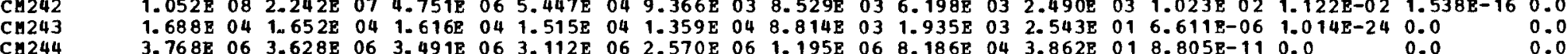

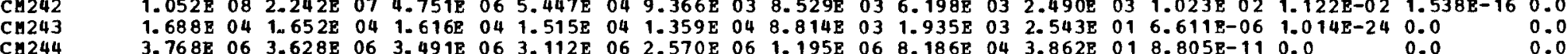


Table 3.1. Shipping schedule for 5-year-old spent fuel

\begin{tabular}{lccc}
\hline Year & $\begin{array}{c}\text { Amount shipped } \\
\text { (MTHM) }\end{array}$ & $\begin{array}{c}\text { Average age } \\
\text { shipped (years) }\end{array}$ & Backlog $^{\text {(MTHM) }}$ (MT \\
\hline 1985 & 2013 & 11 & 5660 \\
1986 & 2220 & 9 & 5026 \\
1987 & 2427 & 8 & 4307 \\
1988 & 2634 & 7 & 3501 \\
1989 & 2840 & 6 & 2674 \\
1990 & 3047 & 6 & 1898 \\
1991 & 3254 & 5 & 1223 \\
1992 & 3461 & 5 & 725 \\
1993 & 3668 & 5 & 385 \\
1994 & 3875 & 5 & 149 \\
1995 & 4024 & 5 & 0 \\
\hline
\end{tabular}

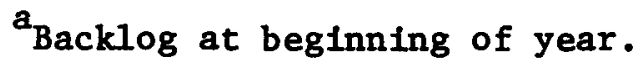

$b_{\text {MTHM }}=$ metric tons of heavy metal. 
Table 3.2. Shipping schedule for 10-year-old spent fuel

\begin{tabular}{lccc}
\hline Year & $\begin{array}{c}\text { Amount shipped } \\
\text { (MTHM) }\end{array}$ & $\begin{array}{c}\text { Average age } \\
\text { shipped (years) }\end{array}$ & Backlog $^{\mathrm{a}}$ (MTHM) \\
\hline 1985 & 567 & 13 & 1009 \\
1986 & 766 & 12 & 1009 \\
1987 & 948 & 11 & 1009 \\
1988 & 1108 & 11 & 1009 \\
1989 & 1262 & 11 & 1009 \\
1990 & 1581 & 11 & 1009 \\
1991 & 1703 & 10 & 807 \\
1992 & 1823 & 10 & 605 \\
1993 & 2009 & 10 & 403 \\
1994 & 2265 & 10 & 201 \\
1995 & 2373 & 10 & 0 \\
\hline
\end{tabular}

$a_{\text {Backlog at beginning of year. }}$

$b_{\text {MTHM }}=$ metric tons of heavy metal. 


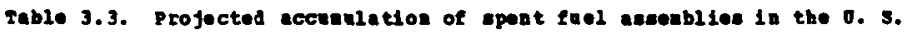

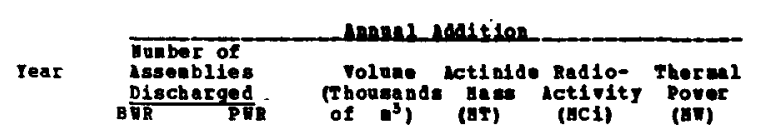

$\begin{array}{rrrrrrr}1976 & 1627 & 1014 & 0.33 & 742 & 2490 & 11 \\ 1977 & 2322 & 1131 & 0.41 & 918 & 4176 & 18 \\ 1978 & 2492 & 1411 & 0.48 & 1073 & 5112 & 22 \\ 1979 & 2626 & 1692 & 0.54 & 1222 & 6079 & 26 \\ 1980 & 2831 & 1863 & 0.59 & 1335 & 6909 & 30 \\ 1981 & 3088 & 2026 & 0.64 & 1453 & 7617 & 33 \\ 1982 & 3296 & 2204 & 0.69 & 1569 & 8256 & 36 \\ 1983 & 3541 & 2509 & 0.77 & 1749 & 9076 & 39\end{array}$

\begin{tabular}{lll}
1984 & 3891 & 2509 \\
\hline & 4905
\end{tabular}

$\begin{array}{lll}1985 & 4405 & 3392 \\ 1986 & 5084 & 3952\end{array}$

$\begin{array}{lll}1987 & 5759 & 4474 \\ 1988 & 6365 & 4909\end{array}$

$\begin{array}{lll}1989 & 6950 & 5314 \\ 1990 & 7553 & 5719\end{array}$

$\begin{array}{lll}1990 & 7553 & 5719 \\ 1991 & 8195 & 6154 \\ 1992 & 8873 & 6621\end{array}$

$\begin{array}{rrr}1992 & 8873 & 6621 \\ 1993 & 9579 & 7110 \\ 1996 & 10305 & 7609\end{array}$

1995

1996

1997

1998
1999

200

2001
2002
2003

2003

2005

2006

2008

2010

201

2013

201

2015
2016
2017

2017
2018
2019

$\begin{array}{lll}2019 & 21277 & 14708 \\ 2020 & 21725 & 14713 \\ 2021 & 21652 & 14859\end{array}$

$\begin{array}{lll}2022 & 21937 & 15328\end{array}$

$2023 \quad 22418 \quad 15665$

$\begin{array}{lll}2024 & 23178 & 15718 \\ 2025 & 23844 & 15892\end{array}$

$\begin{array}{lll}2025 & 23844 & 15892 \\ 2026 & 23692 & 15629\end{array}$

$\begin{array}{lll}2027 & 23313 & 15236 \\ 2028 & 23196 & 15094\end{array}$

$\begin{array}{lll}2028 & 23196 & 15094 \\ 2030 & 23324 & 15080 \\ 23489 & 15180\end{array}$

$\begin{array}{lll}2030 & 23489 & 15180 \\ 2031 & 23601 & 15248\end{array}$ $\begin{array}{rrr}0.77 & 1749 & 9076 \\ 0.88 & 1998 & 10212\end{array}$

$\begin{array}{lll}0.88 & 1998 & 10212 \\ 1.01 & 2297 & 11654\end{array}$

$\begin{array}{lll}1.17 & 2667 & 13435\end{array}$

$\begin{array}{lll}1.33 & 3020 & 15360 \\ 1.46 & 3322 & 17229\end{array}$

1.72369420600

$1.85 \quad 203 \quad 22240$

$\begin{array}{lll}2.00 & 432 & 23966\end{array}$

$\begin{array}{lll}2.15 & 4875 & 25793 \\ 2.30 & 5227 & 27703\end{array}$

$\begin{array}{lll}2.47 & 5600 & 29709 \\ 2.65 & 6005 & 31830\end{array}$

$\begin{array}{lll}2.83 & 6417 & 34067\end{array}$

$\begin{array}{lll}3.01 & 6825 & 36339 \\ 3.19 & 7227 & 38602\end{array}$

$\begin{array}{lll}3.36 & 7622 & 40826 \\ 3.53 & 7988 & 42950\end{array}$

$\begin{array}{lll}3.74 & 8477 & 46384 \\ 3.83 & 8665 & 47564\end{array}$

$3.90 \quad 8820 \quad 48475$

$\begin{array}{lll}3.98 & 9021 & 49605 \\ 4.06 & 9194 & 50565 \\ 4.14 & 9368 & 51530\end{array}$

$\begin{array}{lll}4.14 & 9368 & 51530 \\ 4.24 & 9587 & 52668\end{array}$

$\begin{array}{lll}4.24 & 9587 & 52668 \\ 4.34 & 9802 & 53725\end{array}$

$\begin{array}{rrr}4.41 & 9964 & 54573 \\ 4.50 & 10178 & 55622\end{array}$

$\begin{array}{lll}4.50 & 10178 & 55622 \\ 4.55 & 10301 & 56372\end{array}$

$\begin{array}{lll}4.65 & 10519 & 57369 \\ 4.72 & 10654 & 57845\end{array}$

$\begin{array}{lll}4.62 & 10451 & 57043\end{array}$

$57224 \quad 248$

$\begin{array}{llll}4.57 & 10346 & 56639 & 246 \\ 4.61 & 10428 & 56992 & 247\end{array}$

$\begin{array}{llll}4.63 & 10480 & 57305 & 249\end{array}$

$\begin{array}{llll}4.74 & 10740 & 58574 & 254 \\ 4.85 & 10976 & 59647 & 259 \\ 4.92 & 11135 & 60237 & 262\end{array}$

$\begin{array}{llll}4.92 & 11135 & 60237 & 262 \\ 5.01 & 11331 & 61000 & 265\end{array}$

$\begin{array}{llll}4.95 & 11187 & 60224 & 261 \\ 4.85 & 10944 & 59029 & 256 \\ 4.81 & 10860 & 58581 & 254\end{array}$

$\begin{array}{ll}4.82 & 10880 \\ 4.85 & 10950 \\ 4.87 & 11000\end{array}$

58581

$\mathbf{5 8 6 0 7}$

$\begin{array}{ll}58896 & 256 \\ 59098 & 257\end{array}$ $\begin{array}{lll}3.53 & 7988 & 42950 \\ 3.64 & 8256 & 4488\end{array}$

$\begin{array}{llll}4.60 & 10424 & 57109 & 248\end{array}$

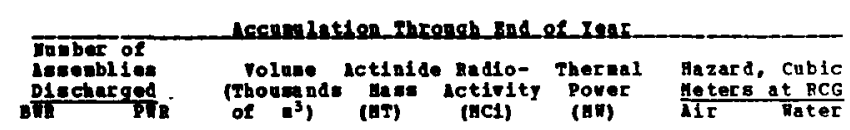

\section{$5705 \quad 2809$}

$\begin{array}{rr}5705 & 2809 \\ 8027 & 3940 \\ 10519 & 5351 \\ 13145 & 7043\end{array}$

1597

19061
22360

22390
29793

9907

10933
13136

15646

34197

18572
21964

25916

$\begin{array}{ll}45041 & 30391 \\ 51406 & 35300 \\ 58356 & 40613\end{array}$

65909
76332

$\begin{array}{ll}65909 & 46332 \\ 74105 & 52486 \\ 82978 & 59107 \\ 92557 & 66217\end{array}$

82978
92557

$\begin{array}{ll}102862 & 73826 \\ 113938 & 81964 \\ 125850 & 90673\end{array}$

$\begin{array}{ll}113938 & 81964 \\ 125854 & 90673\end{array}$

$138627 \quad 99965$
15225

$152265 \quad 109834$

$166711 \quad 120268$

$182015 \quad 131253$

$\begin{array}{ll}198093 & 142740 \\ 210742 & 154617\end{array}$

$231878 \quad 16678$

$\begin{array}{ll}249422 & 179216 \\ 267287 & 191865\end{array}$

$\begin{array}{ll}267287 & 197865 \\ 285525 & 204814\end{array}$

$304130 \quad 21800$

323090
342764
345091

342764
363227
3

$383965 \quad 272970$

$\begin{array}{ll}383965 & 272970 \\ 405242 & 287302\end{array}$

426209302037

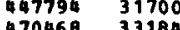

$\begin{array}{ll}490468 & 337848 \\ 492245 & 336593\end{array}$

513213
561666

$\begin{array}{ll}533956 & 376763 \\ 555233 & 391471 \\ 576958 & 005788\end{array}$

$\begin{array}{ll}598610 & 421043 \\ 620547 & 43637\end{array}$

$642966 \quad 452036$

$666144 \quad \$ 6775$

713679

$736992 \quad 514511$

760188529605

$783511 \quad 544693$

$\begin{array}{ll}807000 & 559873 \\ 830602 & 575120\end{array}$

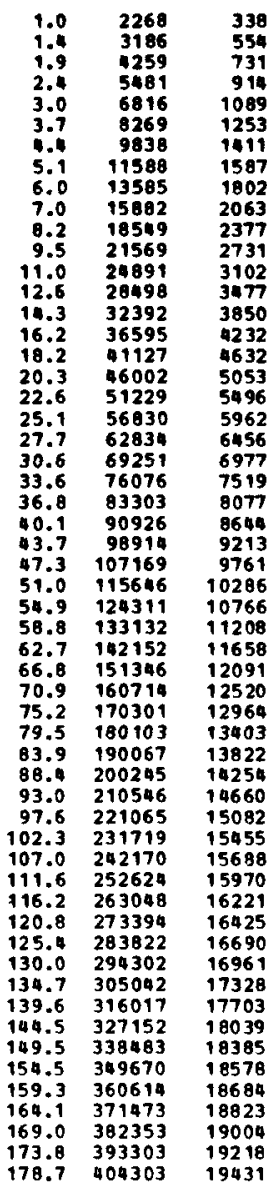

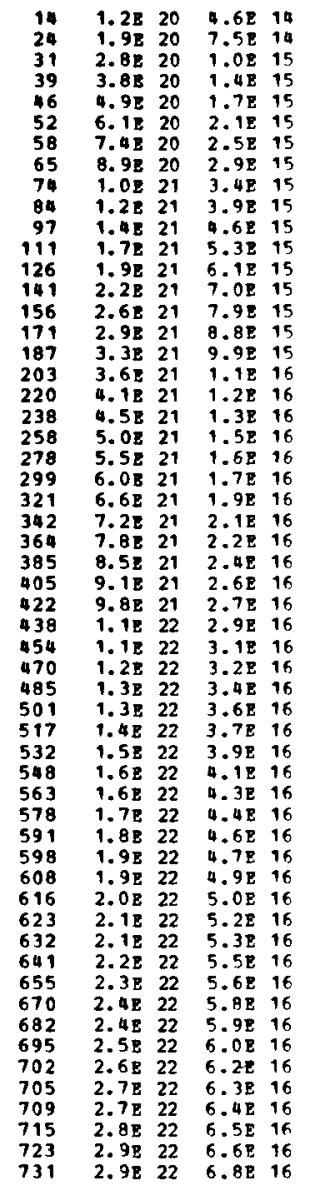

$\begin{array}{ll}830602 & 575120 \\ 830602 & 575120 \\ 830602 & 575120 \\ 830602 & 575120 \\ 830602 & 575120 \\ 830602 & 575120 \\ 830602 & 575120 \\ 830602 & 575120 \\ 830602 & 575120\end{array}$

$178.7 \quad 404303$

$\begin{array}{rr}178.7 & 404303 \\ 178.7 & 404303\end{array}$

178.7404303

$\begin{array}{rr}178.7 & 404303 \\ 178.7 & 404303 \\ 178.7 & 404303\end{array}$

$178.7 \quad 404303$

$\begin{array}{rr}178.7 & 404303 \\ 178.7 & 404303\end{array}$ $\begin{array}{rrrrrr}10381 & 93 & 1.7 E & 22 & 5.8 E & 15 \\ 1527 & 47 & 1.18 & 22 & 3.78 & 14 \\ 652 & 21 & 6.68 & 29 & 1.4 E & 14 \\ 291 & 9 & 4.4 \mathrm{E} & 21 & 5.78 & 13 \\ 180 & 5 & 2.7 \mathrm{~B} & 21 & 3.6 \mathrm{E} & 13 \\ 75 & 2 & 1.0 \mathrm{E} & 29 & 2.0 \mathrm{E} & 13 \\ 29 & 0 & 1.5 \mathrm{E} & 20 & 2.0 \mathrm{E} & 13 \\ 13 & 0 & 3.3 \mathrm{~B} & 19 & 2.2 \mathrm{E} & 13 \\ 8 & 0 & 2.5 \mathrm{~B} & 19 & 1.0 \mathrm{E} & 13\end{array}$ 
Table 3.4. Grans of light elenents accunulated In the 0 . S.

$\begin{array}{llllllllll}1980 & 1985 & 1990 & 1995 & 2000 & 2005 & 2010 & 2015 & 2020 & 2030\end{array}$

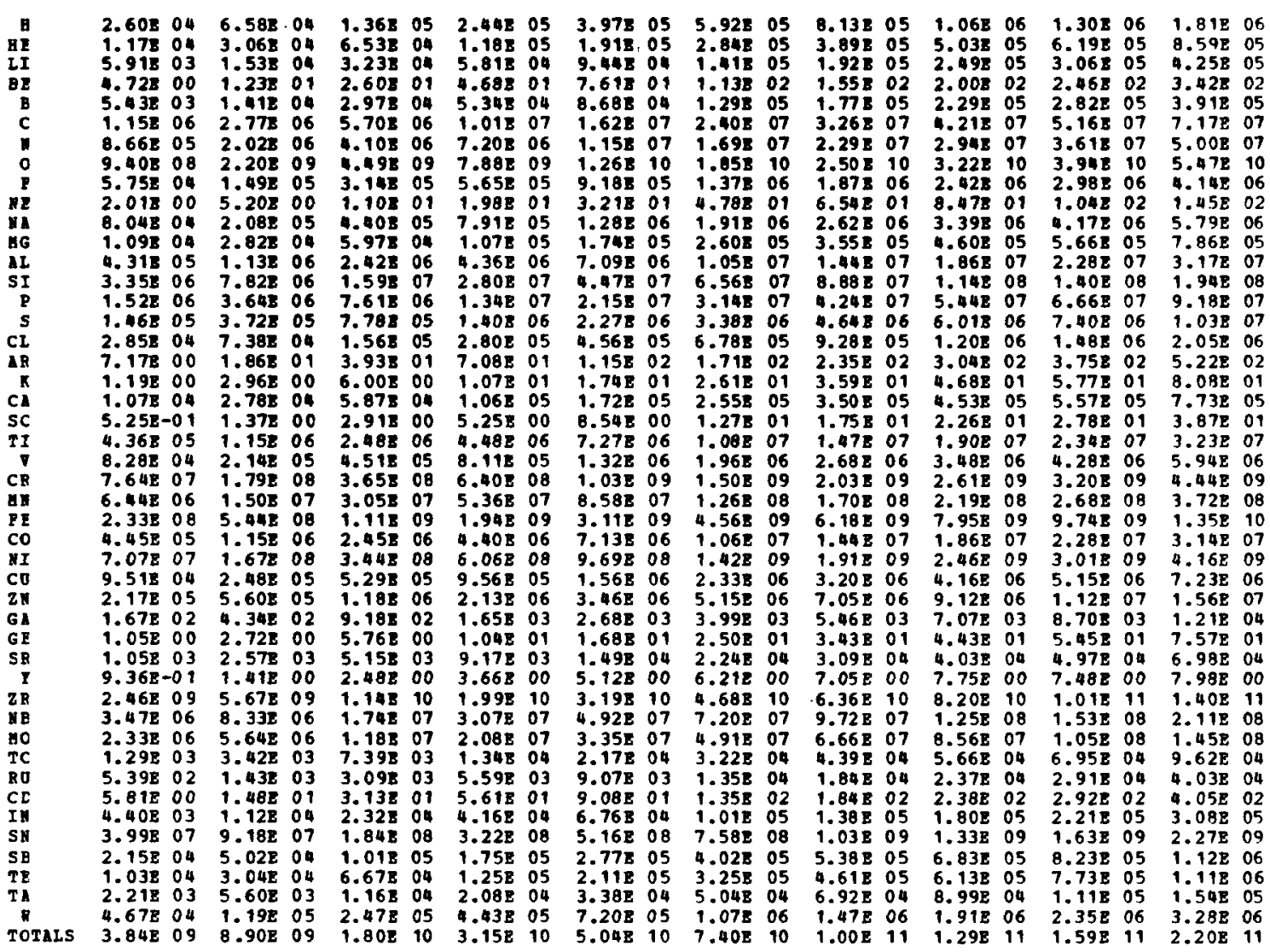


Table 3.5. Graes of fission-product elements accunlated in the 0.5 .

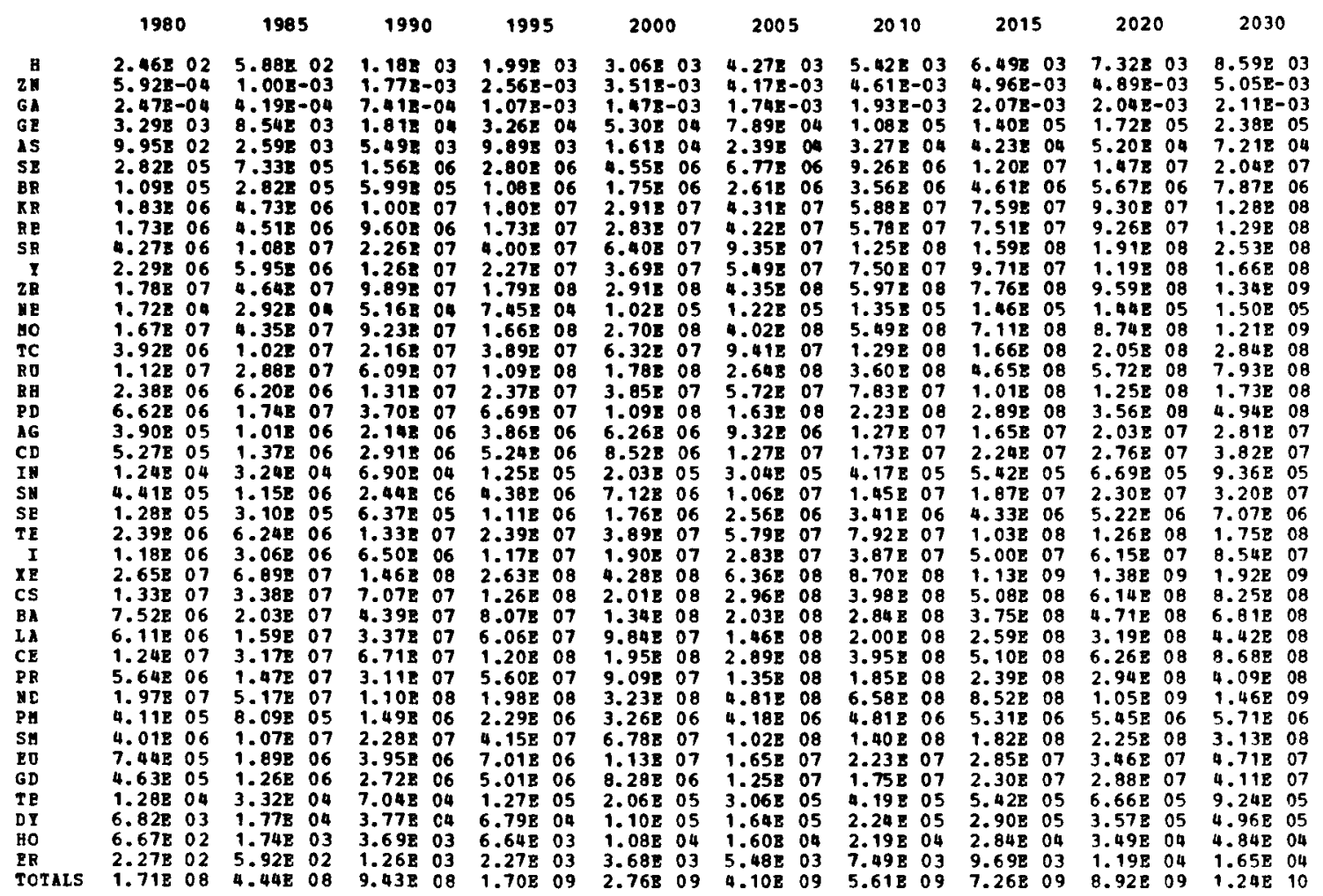

Table 3.6. Grans of heavy elesents accunulated in the 0 . $S$.

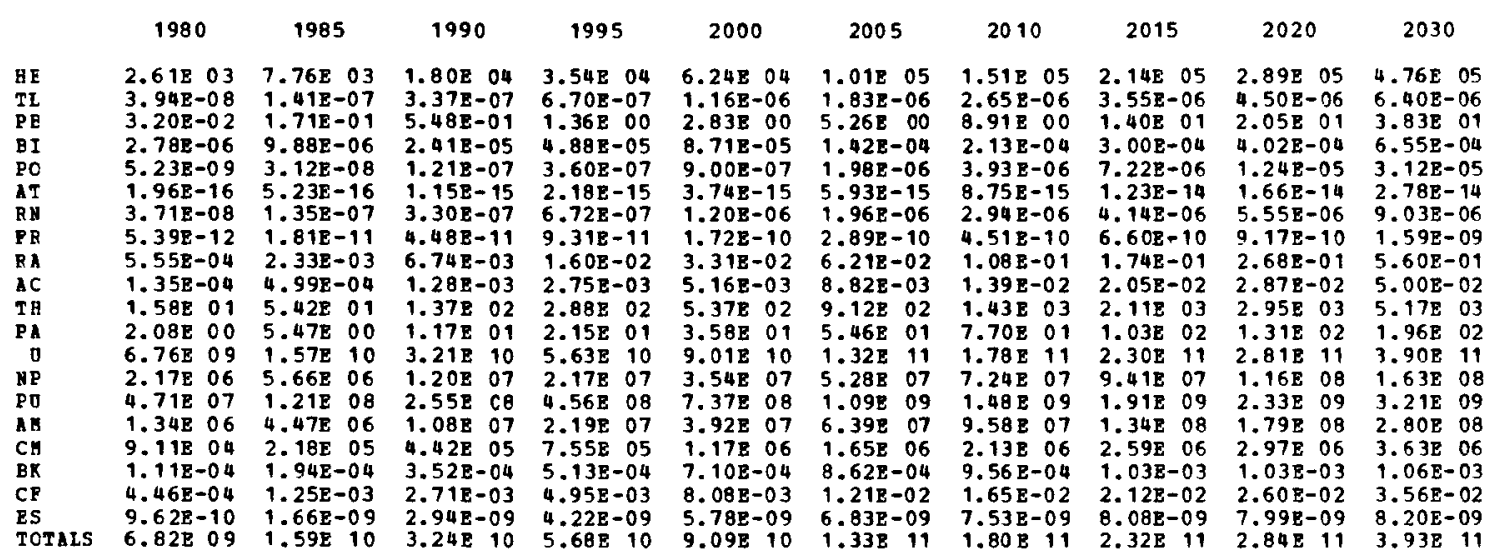


Table 3.7. Curies of light-elenent isotopes accunulated in the 0.5 .

1980

1985

$$
1990
$$

1995

2000

2005

2010

2015

2020

2030

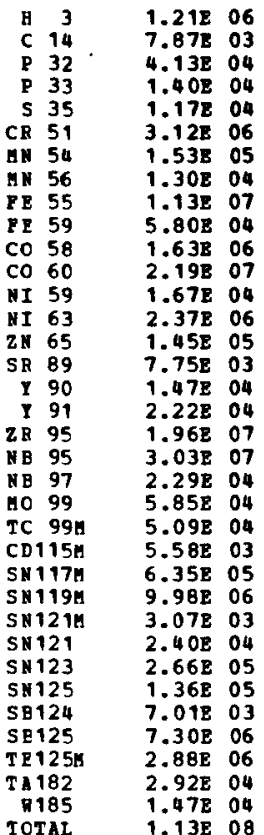

$2.88 \mathrm{E}$ 06 $5.74 \mathrm{E}$ 06

$\begin{array}{llll}9.735 & 06 & 1.498 & 07 \\ 7.755 & 04 & 1.268 & 05\end{array}$

$\begin{array}{lllllll}2.095 & 07 & 2.658 & 07 & 3.185 & 07\end{array}$ $\begin{array}{llllll}1.87 B & 05 & 2.568 & 05 & 3.328 & 05 \\ & 3.35\end{array}$

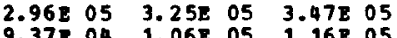
$7.26 \mathrm{E} 04 \quad 1.29 \mathrm{~B} \quad 05$ 2.152 o4 3.7880 1.978 O4 3.48E 04 5.438 O6 $9.63 \mathrm{E} 06$ $1.84 \mathrm{E}$ OS $2.51 \mathrm{E} 05$ $5.56 \mathrm{~B}$ O4 $7.76 \mathrm{E}$ O4 $9.37 \mathrm{~B}$ O4 $1.06 \mathrm{E}$ 0 $5.03 E$ O4 6.938 O4 $\begin{array}{llll}1.38 E & 07 & 1.89 E & 07\end{array}$ $\begin{array}{llllll}2.238 & 07 & 2.45 B & 07 & 2.62 E & 07\end{array}$ $2.30204 \quad 4.07504$ \begin{tabular}{lllllllll}
05 & 9.438 & 05 & 1.158 & 06 & 1.298 & 06 & $1.40 \mathrm{~B}$ & 06 \\
\hline
\end{tabular} $2.30204 \quad 4.07804 \quad 5.828$ o4 7.948 O4 $\begin{array}{llll}2.31 \mathrm{E} & 07 & 4.33 \mathrm{E} & 07 \\ 1.028 & 05 & 1.308 & 05\end{array}$ $\begin{array}{llllllllll}6.63 \mathrm{E} & 07 & 9.38 \mathrm{E} & 07 & 1.19 \mathrm{E} & 08 & 1.36 \mathrm{~B} & 08 & 1.50 \mathrm{E} & 08\end{array}$

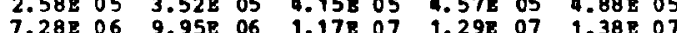
$7.28 \mathrm{E} 06$ 9.95E 06 $2.87=06$ $2.87806 \quad 5.09206$ 4.928079 .57807 4.452 O4 9.6420 6.25E $06 \quad 1.34207$ 1.748052 .26808 $1.74 E 05 \quad 2.838$ o5 $\begin{array}{llll}2.40 E & 07 & 3.86 B & 07 \\ 6.45 E & 05 & 8.92 B & 05\end{array}$ 2.478 on 2.0580 7.638
$07 \quad 9.71 E$ $.19805 \quad 5.728$ 05 7.37805 6.45E 05 8.92B 05 1.08E 06 1.20E 06 $1.30 \mathrm{E} 06$ 2.05204 3.03E O4 4.24E O4 $2.22 \mathrm{E} 04 \quad 3.89 \mathrm{~B}$ O4 $5.75 \mathrm{E}$ O4 $8.05 \mathrm{E}$ 04 $.14 \mathrm{~B} 04 \quad 5.84 \mathrm{E} 04 \quad 6.42 \mathrm{E} \quad 0$ $9.76 \mathrm{E}$ 04 1.118 O5 $1.22 \mathrm{E} 05$ 5.618 o $4.91807 \quad 8.67807$ $3.73804 \quad 6.57804$ 1.028 o5 1.81805 0.898042 .58805 $0.892041 .58 \mathrm{~B} 05$ 8.678 O4 1.218 O5 $1.13 \mathrm{~B} 081.35 \mathrm{E} 0 \mathrm{~B}$ $1.26808 \quad 1.74808$ 9.55 OH $1.32 \mathrm{~B}$ 05 $1.59 \mathrm{~B} 08$ $2.59 \mathrm{E}$ OS $3.54 \mathrm{E} 05$ $2.26 \mathrm{~B} 05 \quad 3.08805$ $05 \quad 4.18 \mathrm{E} 05 \quad 4.60 \mathrm{E} \quad 05 \quad 4.92 \mathrm{E} \quad 05$ $\begin{array}{llll}2.338 & 08 & 2.532 & 08\end{array}$ \begin{tabular}{lll}
05 & $1.91 \mathrm{E}$ & 05 \\
\hline
\end{tabular} $3.928040 .3404 \quad 4.28805$ 2.408 O4 $3.30 \mathrm{E}$ O4 3.928 O4 $4.34 \mathrm{E}$ O4 4.678 O4 $\begin{array}{llllllllllll}2.64 E & 06 & 3.65 E & 06 & 4.37 E & 06 & 4.88 E & 06 & 5.30 E & 06\end{array}$ $\begin{array}{llllllllll}4.28 \mathrm{E} & 07 & 5.96 \mathrm{E} & 07 & 7.27 \mathrm{E} & 07 & 8.13 \mathrm{E} & 07 & 8.85 \mathrm{E} & 07 \\ 2.79 \mathrm{E} & 04 & 4.49 \mathrm{E} & 04 & 6.61 \mathrm{E} & 04 & 8.94 \mathrm{~B} & 04 & 1.148 & 05\end{array}$ $\begin{array}{lllllllllll}2.79 \mathrm{E} & 04 & 4.49 \mathrm{E} & 04 & 6.61 \mathrm{E} & 04 & 8.94 \mathrm{E} & 04 & 1.14 \mathrm{E} & 05 \\ 9.99 \mathrm{E} & 04 & 1.38 \mathrm{E} & 05 & 1.65 \mathrm{~B} & 05 & 1.85 \mathrm{E} & 05 & 2.00 \mathrm{E} & 05\end{array}$ $\begin{array}{llllllllll}9.998 & 04 & 1.38 \mathrm{E} & 05 & 1.65 \mathrm{E} & 05 & 1.85 \mathrm{E} & 05 & 2.00 \mathrm{E} & 05 \\ 1.11 \mathrm{E} & 06 & 1.54 \mathrm{E} & 06 & 1.85 \mathrm{~B} & 06 & 2.078 & 06 & 2.25 \mathrm{E} & 06\end{array}$

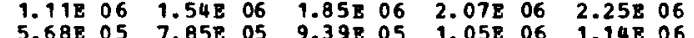
$\begin{array}{llllllllll}5.68 E & 05 & 7.85 E & 05 & 9.398 & 05 & 1.05 E & 06 & 1.14 E & 06 \\ 2.92 E & 04 & 4.04 E & 04 & 4.83 E & 04 & 5.40 E & 04 & 5.86 E & 04\end{array}$ $\begin{array}{llllllllll}2.92 \mathrm{E} & 04 & 4.04 \mathrm{E} & 04 & 4.83 \mathrm{E} & 04 & 5.40 \mathrm{E} & 04 & 5.86 \mathrm{E} & 04 \\ 3.92 \mathrm{~B} & 07 & 5.61 \mathrm{~B} & 07 & 7.25 \mathrm{~B} & 07 & 8.40 \mathrm{E} & 07 & 9.33 \mathrm{~B} & 07\end{array}$ $\begin{array}{llllllllllll}1.56 \mathrm{E} & 07 & 2.24 \mathrm{E} & 07 & 2.90 \mathrm{E} & 07 & 3.37 \mathrm{E} & 07 & 3.75 \mathrm{E} & 07\end{array}$

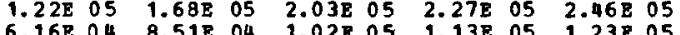
\begin{tabular}{llllllllll}
$6.16 \mathrm{E}$ & 04 & $8.51 \mathrm{E}$ & 04 & $1.02 \mathrm{E}$ & 05 & $1.13 \mathrm{E}$ & 05 & $1.23 \mathrm{E}$ & 05 \\
\hline & & &
\end{tabular} $3.58807 \quad 4.21807$ $4.08 \mathrm{~B} \quad 05 \quad 5.66 \mathrm{E} \quad 05$ $\begin{array}{lllll}3.45 \mathrm{E} & 05 & 3.52 \mathrm{E} & 05\end{array}$ $1.13805 \quad 1.19805$ $9.73804 \quad 1.01805$ $\begin{array}{lllll}2.60 \mathrm{E} & 07 \quad 2.66 \mathrm{E} & 07\end{array}$ $1.39 \mathrm{E} 06 \quad 1.45 \mathrm{E} 06$ $1.0905 \quad 111805$ 1.54808 1.598 08 1. 04 05 T.95E 05 1.378 07 1.958 05 4. $9.06 \mathrm{E} 05 \quad 1.25 \mathrm{E} 06$ $\begin{array}{llll}1.18 \mathrm{E} & 08 & 1.57 \mathrm{E} & 08\end{array}$ $1.28 \mathrm{E} 06 \quad 1.33 \mathrm{E}$ 06 $6.20804 \quad 6.61 \mathrm{E}$ 04 $1.18 \mathrm{E}$ 05 1.26E 05 $1.77 \mathrm{E}$ 05 1.89E 05 $\begin{array}{lllll}1.608 & 08 & 1.68 \mathrm{~B} & 08\end{array}$ $2.48 E \quad 08 \quad 2.59 E 08$ $1.87 \mathrm{E} 05 \quad 1.95 \mathrm{E} 05$ $4.88 \mathrm{E} 05 \quad \mathrm{M} .99 \mathrm{E} 05$ 4.248 $05 \quad 4.34 E_{05}$ 611044.76804 5.18D $06 \quad 5.41806$ . 71807 S.41E 06 . 380 05 9.12807 $1.86 \mathrm{E} 05$ 1.05E 05 $2.20 \mathrm{E} 06 \quad 2.30 \mathrm{E} 06$ $1.11 \mathrm{E} 06 \quad 1.16 \mathrm{E} 06$ .73E 04 5.99E 04 $\begin{array}{llll}5.58 E & 1.01 \mathrm{E} 08\end{array}$ $\begin{array}{lll}.85 E & 07 & 4.08 \mathrm{E} 07\end{array}$ 2.41E 05 2.52E 05 $20 \mathrm{E} 05 \quad 1.25 \mathrm{E} 05$ 
Table 3.8. Curies of fission-prodnct laotopes accunulated in the 0 . $S$.

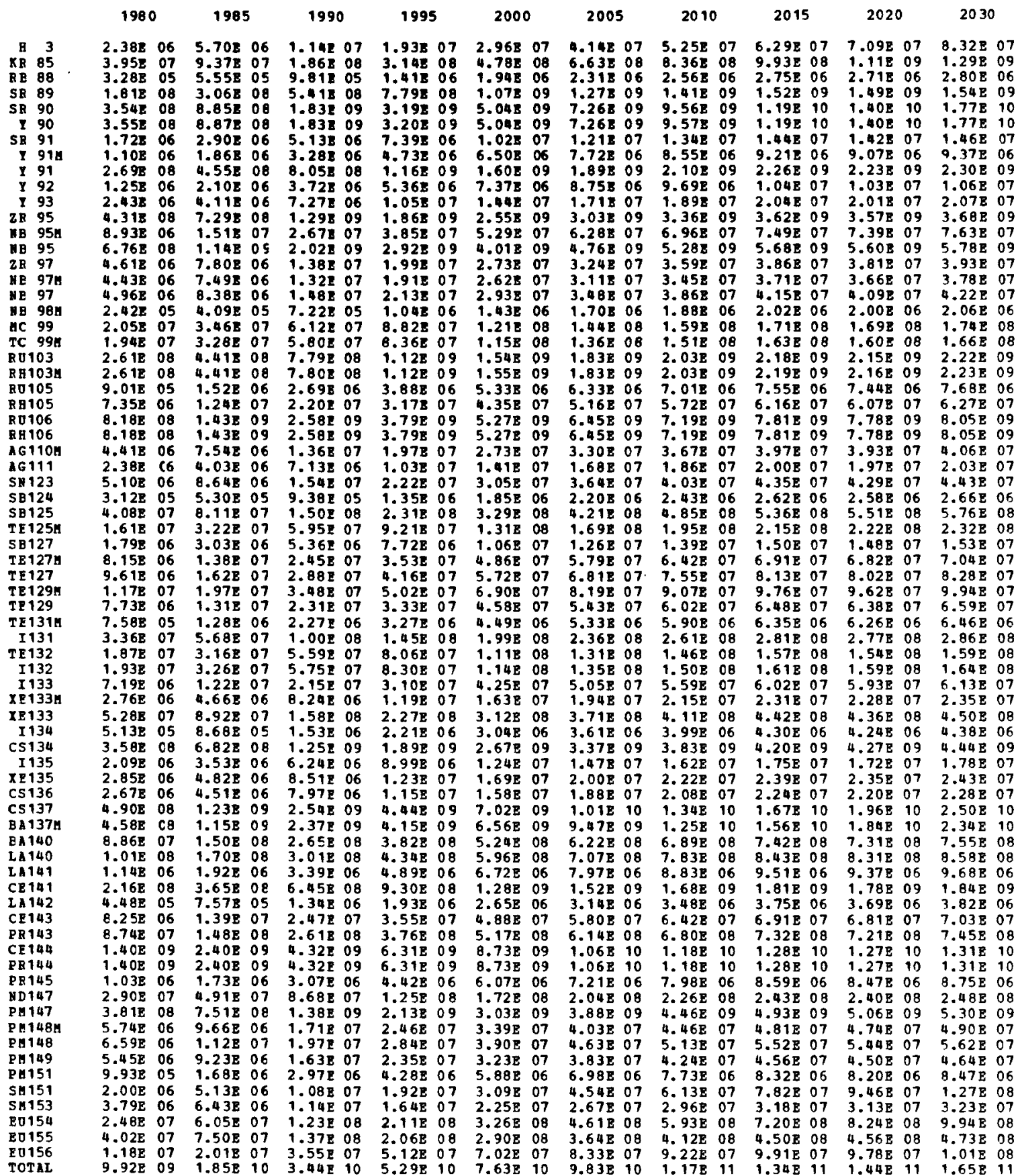


Table 3.9. Curies of heary-elenent 1 sotopes accululated in the 0.5 .

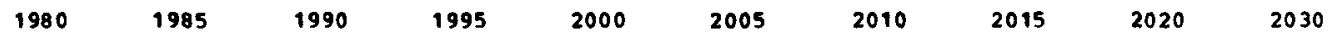

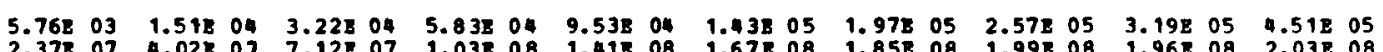

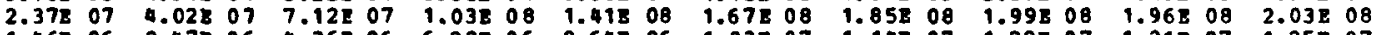

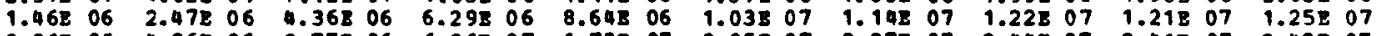
$\begin{array}{lllllllllllllllllllllll}2.918 & 06 & 4.968 & 06 & 8.778 & 06 & 1.268 & 07 & 1.738 & 07 & 2.058 & 07 & 2.272 & 07 & 2.448 & 07 & 2.418 & 07 & 2.48 \mathrm{~B} & 07\end{array}$ $\begin{array}{lllllllllllllllllllll}2.128 & 08 & 3.588 & 08 & 6.328 & 08 & 9.128 & 08 & 1.258 & 09 & 1.498 & 09 & 1.658 & 09 & 1.788 & 09 & 1.758 & 09 & 1.818 & 09\end{array}$ $\begin{array}{lllllllllllllllllllllllllll}1.108 & 07 & 2.83 E & 07 & 5.988 & 07 & 1.078 & 08 & 1.728 & 08 & 2.538 & 08 & 3.42 E & 08 & 4.368 & 08 & 5.28 E & 08 & 7.108 & 08\end{array}$ $\begin{array}{lllllllllllllllllllllll}1.638 & 06 & 4.238 & 06 & 8.94 E & 06 & 1.618 & 07 & 2.618 & 07 & 3.898 & 07 & 5.328 & 07 & 6.898 & 07 & 8.478 & 07 & 1.18 \mathrm{~B} & 08\end{array}$

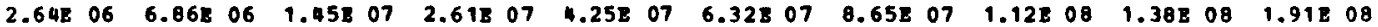

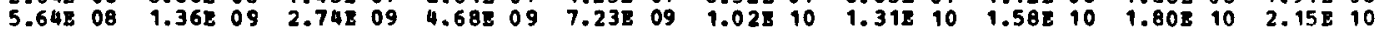

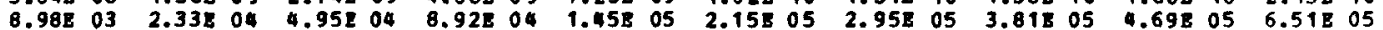

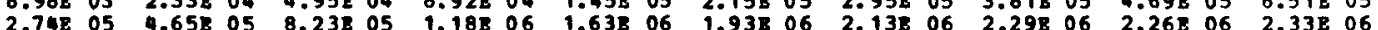

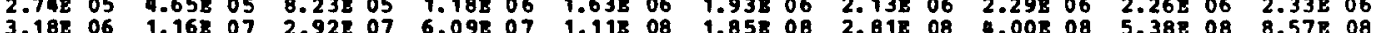

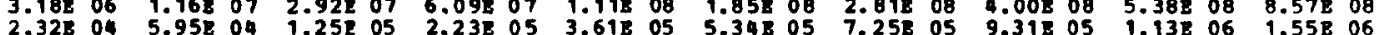

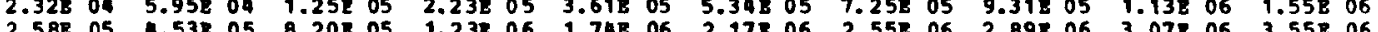

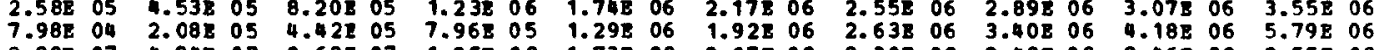

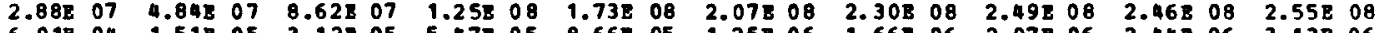

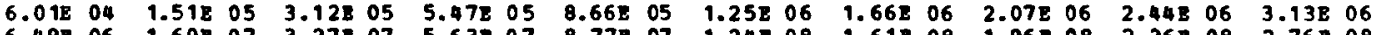

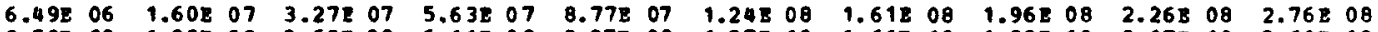

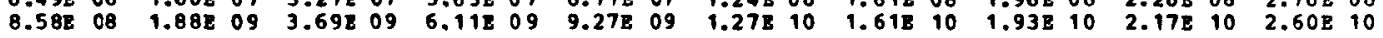


Table 3.10. Watts of 11 ght-elenent 1 eotopes acconulated in the 0.5 .

$\begin{array}{llllllllll}1980 & 1985 & 1990 & 1995 & 2000 & 2005 & 2010 & 2015 & 2020 & 2030\end{array}$

$1.02102 \quad 2.04502$

$\begin{array}{lllll}1.468 & 02 & 5.318 & 02\end{array}$

.42802

$9.42 \mathrm{~B} \quad 02$

1.13203

2020

$\begin{array}{llll}6.048 & 00 & 1.28 E & 01 \\ 2.998 & 02 & 5.308 & 02\end{array}$

$\begin{array}{llll}3.302 & 01 & 3.735 & 01 \\ 7.588 & 02 & 1.048 & 03\end{array}$

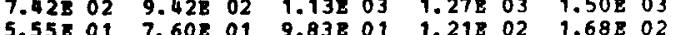

9.7180001 .70801

$\begin{array}{llllllll}1.588 & 02 & 1.048 & 03 & 1.228 & 03 & 1.34 E & 03\end{array}$

$\begin{array}{llllll}9.83 E & 0 & 1.21 E & 02 & 1.68 E & 02 \\ 1.438 & 03 & 1.228 & 03 & 1.45 E & 03\end{array}$

$5.59800 \quad 9.91800$

1.438011 .97801

$\begin{array}{llllllllll}4.22 \mathrm{E} & 01 & 4.78 \mathrm{E} & 01 & 5.24 \mathrm{E} & 01 & 5.07 \mathrm{E} & 01 & 5.38 \mathrm{E} & 01 \\ 2.35 \mathrm{E} & 01 & 2.61 \mathrm{E} & 01 & 2.81 \mathrm{E} & 01 & 2.77 \mathrm{E} & 01 & 2.87 \mathrm{E} & 01\end{array}$

2.408 O4 4.268 O4

6.108 o 8.348 O4 9.848 o4 2.61801

2.08E $03 \quad 3.73803$

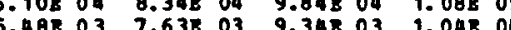

$1.16 \mathrm{E}$ 05 $1.15 \mathrm{E}$ 05 $1.18 \mathrm{E}$ 05

3.598026 .36802

$5.40803 \quad 7.63503$

$1.13 E$ O4 $1.12 E$ O4 $1.17 \mathrm{E} 04$

3.018 O4 5.64804

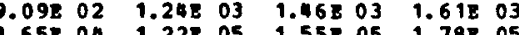

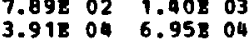

$2.00503 \quad 2.74503$

$\begin{array}{llll}3.918 & 04 & 6.958 & 04 \\ 7.708 & 05 & 1.508 & 06\end{array}$

9.958 O4 $1.36 \mathrm{E} 05$

051.95805

$\begin{array}{llll}1.70 \mathrm{E} & 03 & 1.74 \mathrm{E} 03\end{array}$

1.95E 05 2.01E 05 2.08E 05

7.70805 1.508 06

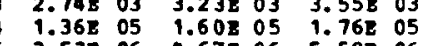

$3.79 \mathrm{E} 03$

$\begin{array}{llllllllll}1.008 & 03 & 2.158 & 03 & 3.858 & 03 & 6.188 & 03\end{array}$

$1.88 \mathrm{~B} 05$

\begin{tabular}{llll}
$3.76 \mathrm{E}$ & 03 & $3.85 \mathrm{E}$ & 03 \\
\hline
\end{tabular}

$\begin{array}{llllllllll}4.672 & 06 & 5.59 E & 06 & 6.34 \mathrm{E} & 06 & 6.77 \mathrm{E} & 06 & 7.26 \mathrm{E} & 06 \\ 9.07 \mathrm{E} & 03 & 1.22 \mathrm{E} & 04 & 1.55 \mathrm{E} & \text { O4 } & 1.88 \mathrm{E} & \text { O4 } & 2.52 \mathrm{E} & 04\end{array}$

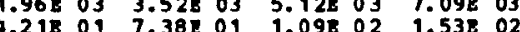

17.38201

$\begin{array}{llllll}1.098 & 02 & 1.538 & 02 & 1.858 & 02\end{array}$

$9.52 E 03$

$1.082042 .52 \mathrm{E} 04$

$1.28802 \quad 2.23802$

$3.39802 \quad 4.74802$

2.10E 02

$2.31 \mathrm{E}$ 1.02E O4 $1.06 \mathrm{E} \mathrm{O4}$

1.678 05 2.948 05

. 278 05 5.918

$2.23 \mathrm{E} \quad 02 \quad 2.38 \mathrm{E} \quad 02$

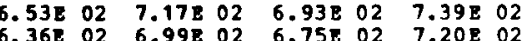

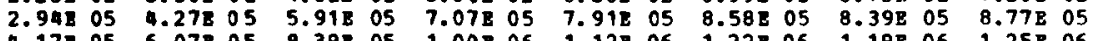

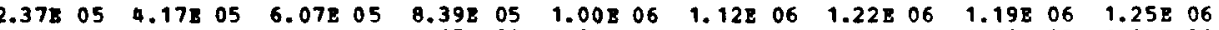

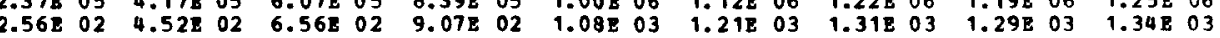

$4.012027 .11 \mathrm{E} 02$ 1.025 $03 \quad 139803$

$1.49802 \quad 2.6480203 .79502 \quad 5.17 \mathrm{E} 02$

$1.64 \mathrm{E} 03 \quad 1.80 \mathrm{E} 03$

$\begin{array}{llllll}1.93 \mathrm{E} & 03 & 1.91 \mathrm{E} & 03 & 1.96 \mathrm{E} & 03 \\ 7.18 \mathrm{E} & 02 & 7.12 \mathrm{E} & 02 & 7.28 \mathrm{E} & 02\end{array}$

$3.79 \mathrm{E} \quad 02 \quad 5.17 \mathrm{E} \quad 02$

$6.10 \mathrm{~B} \quad 02 \quad 6.71 \mathrm{~B} \quad 02$

1.47:01 6.14801

$8.85 \mathrm{E} 01 \quad 1.22 \mathrm{E} 02 \quad 1.44 \mathrm{E} 02$

$.94 E 03 \quad 3.42203$

$4.97 \mathrm{E} 03 \quad 6.87 \mathrm{E} 03$

.

2.55E 01 C 1.65201

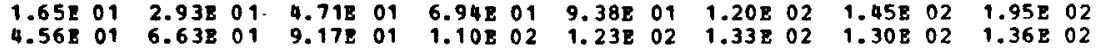

$8.21 \mathrm{E} 03$

$1.60 \mathrm{~B} 02$

$\begin{array}{ll}7.188 & 02 \\ 1.728 & 02\end{array}$

$\begin{array}{rlll}12 \mathrm{E} & 02 & 7.28 \mathrm{E} & 02\end{array}$

1.75E 03 $9.70 \mathrm{E} 02 \quad 1.75 \mathrm{E} 02$

$4.29 \mathrm{E}$ O4 $4.67 \mathrm{E}$ O4 $4.60 \mathrm{E} 04 \quad 4.81 \mathrm{E}$ O4

$\begin{array}{lllllll}2.602 & 03 & 3.798 & 03 & 5.25 E & 03\end{array}$

$1.10 \mathrm{E} 02$

$1.33 \mathrm{E} 0$

$1.30 \mathrm{E} 02 \quad 1.36 \mathrm{E} 02$

6.31E 03 7.05E 03

$\begin{array}{llllll}7.65 E & 03 & 7.50 E & 03 & 7.84 E & 03\end{array}$

$\begin{array}{cccc}5.55 \mathrm{E} & 03 & 6.20 \mathrm{E} & 03\end{array}$

$\begin{array}{llllllll}6.72 \mathrm{E} & 03 & 6.58 \mathrm{E} & 03 & 6.87 \mathrm{E} & 03\end{array}$

5.672 o4 1.028 O5

$1.59805 \quad 2.27 \mathrm{E}$ 05

$6.30 \mathrm{~B} 02 \quad 7.04 \mathrm{~B} 02$

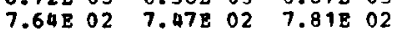

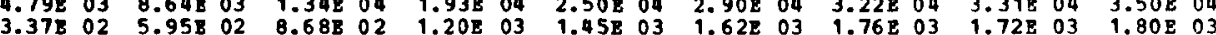

$2.28 \mathrm{E} 01 \quad 4.03801 \quad 5.84801 \quad 8.07801 \quad 9.65801$

1.08202

1.76803

$1.72 \mathrm{E} 03$

$1.19 \mathrm{E} 02$

1.35E $06 \quad 2.53 \mathrm{E}$ O6

$3.92806 \quad 5.638$ 06

$7.22 \mathrm{E} 06$

$9.45 \mathrm{E} 06 \quad 9.85 \mathrm{E}$ 06 $1.05 \mathrm{E} 07$ 
Table 3.11. Natts of fission-product Isotopes accunulated in the 0 . $S$.

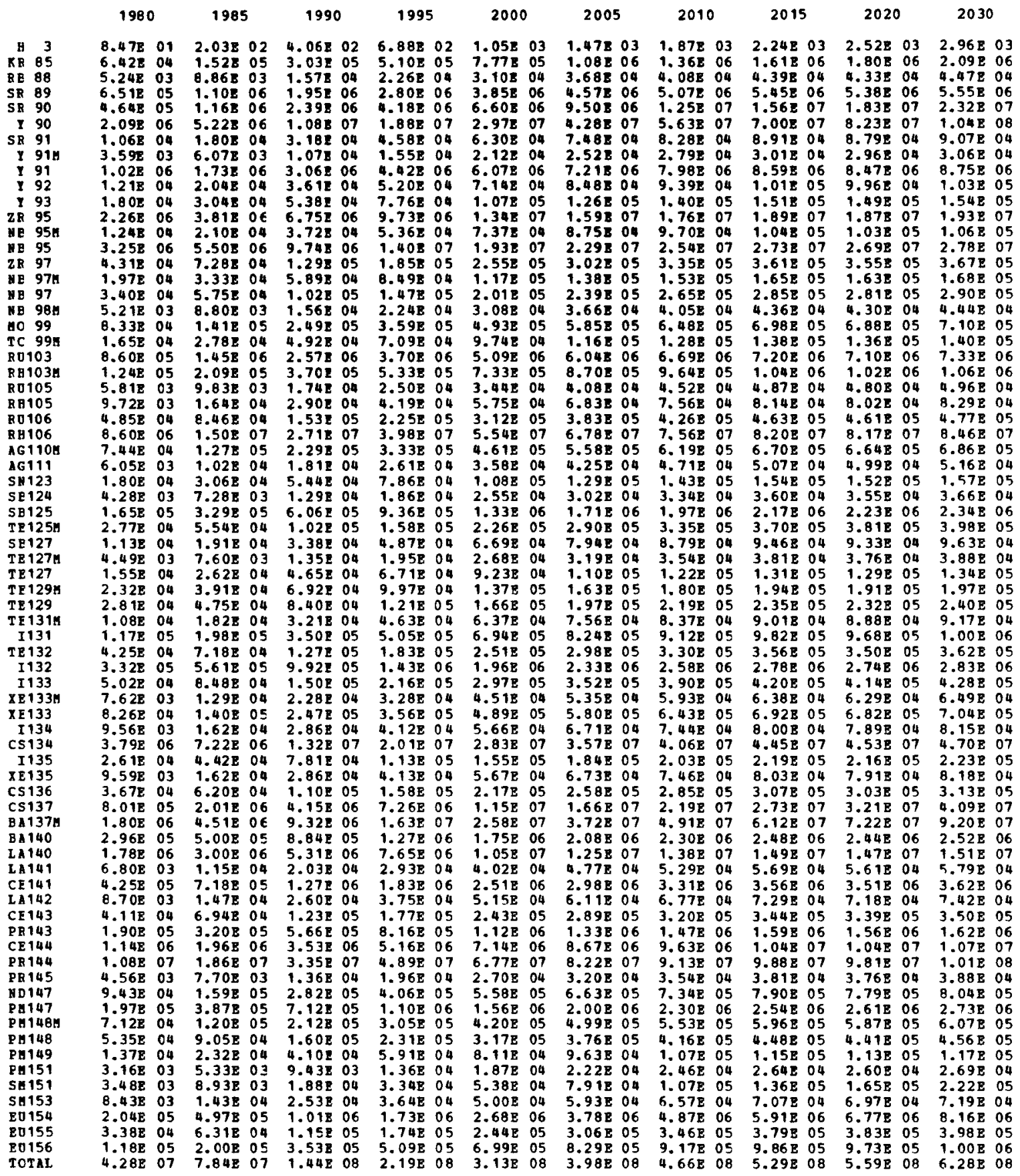


Table 3.12. Watts of heary-alenent lsotopes accunulated in the 0.5 .

$\begin{array}{llllllllll}1980 & 1985 & 1990 & 1995 & 2000 & 2005 & 2010 & 2015 & 2020 & 2030\end{array}$

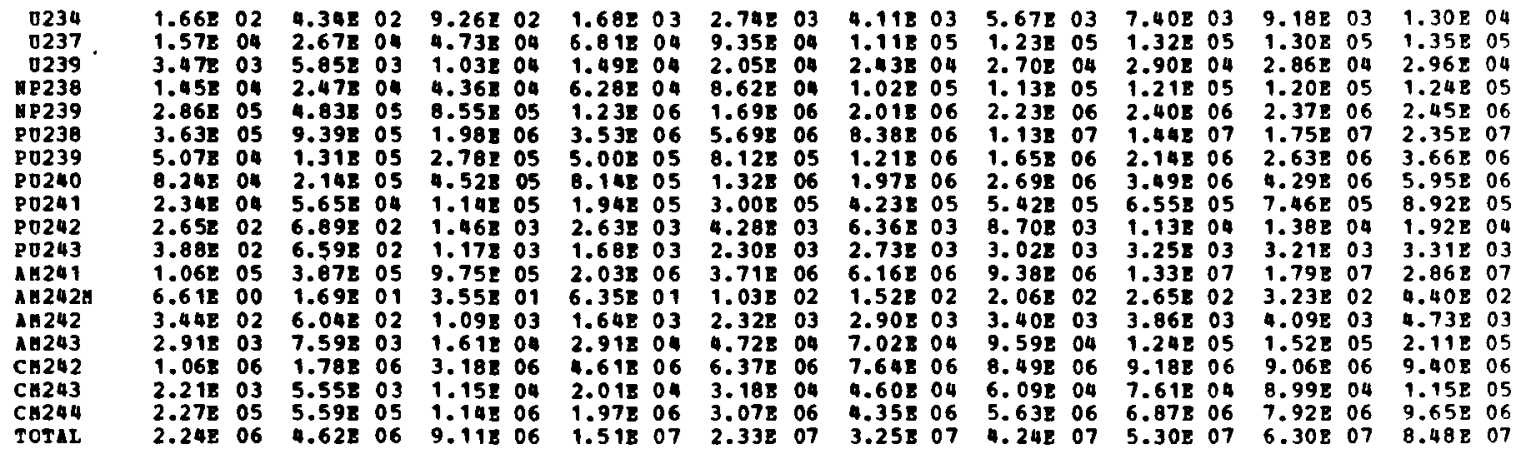


Table 3.13. Ingesticn toxiclty $\left(\mathrm{m}^{3}\right.$ water) of light-elenent 1 sotopes accunulated in the 0 . $S$.

$\begin{array}{llllllllll}1980 & 1985 & 1990 & 1995 & 2000 & 2005 & 2010 & 2015 & 2020 & 2030\end{array}$

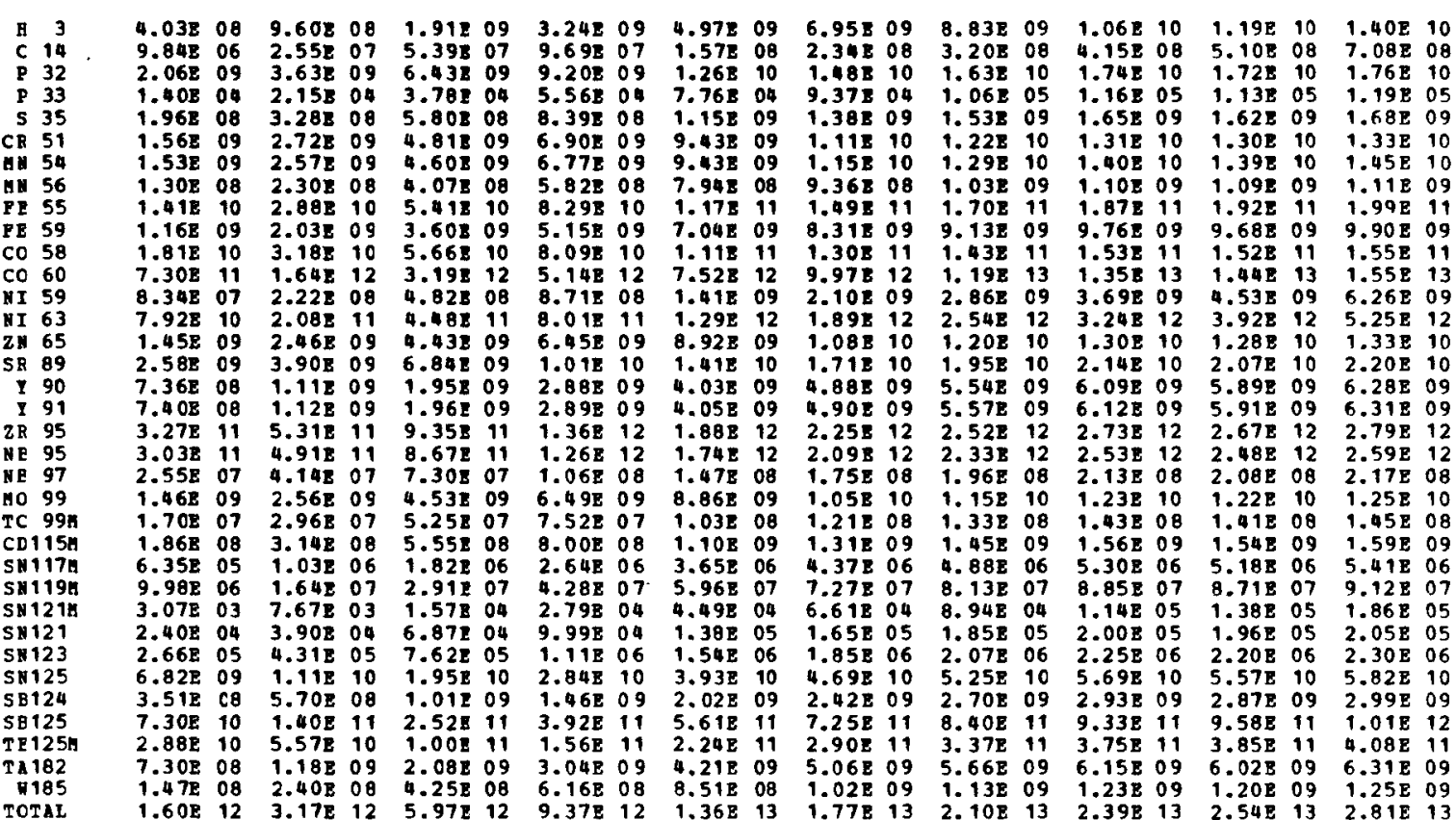


Table 3.14. Ingestion toxicity ( $a^{3}$ vater) of fission-product isotopes accunulated in the $U$. S.

$\begin{array}{llllllllll}1980 & 1985 & 1990 & 1995 & 2000 & 2005 & 2010 & 2015 & 2020 & 2030\end{array}$

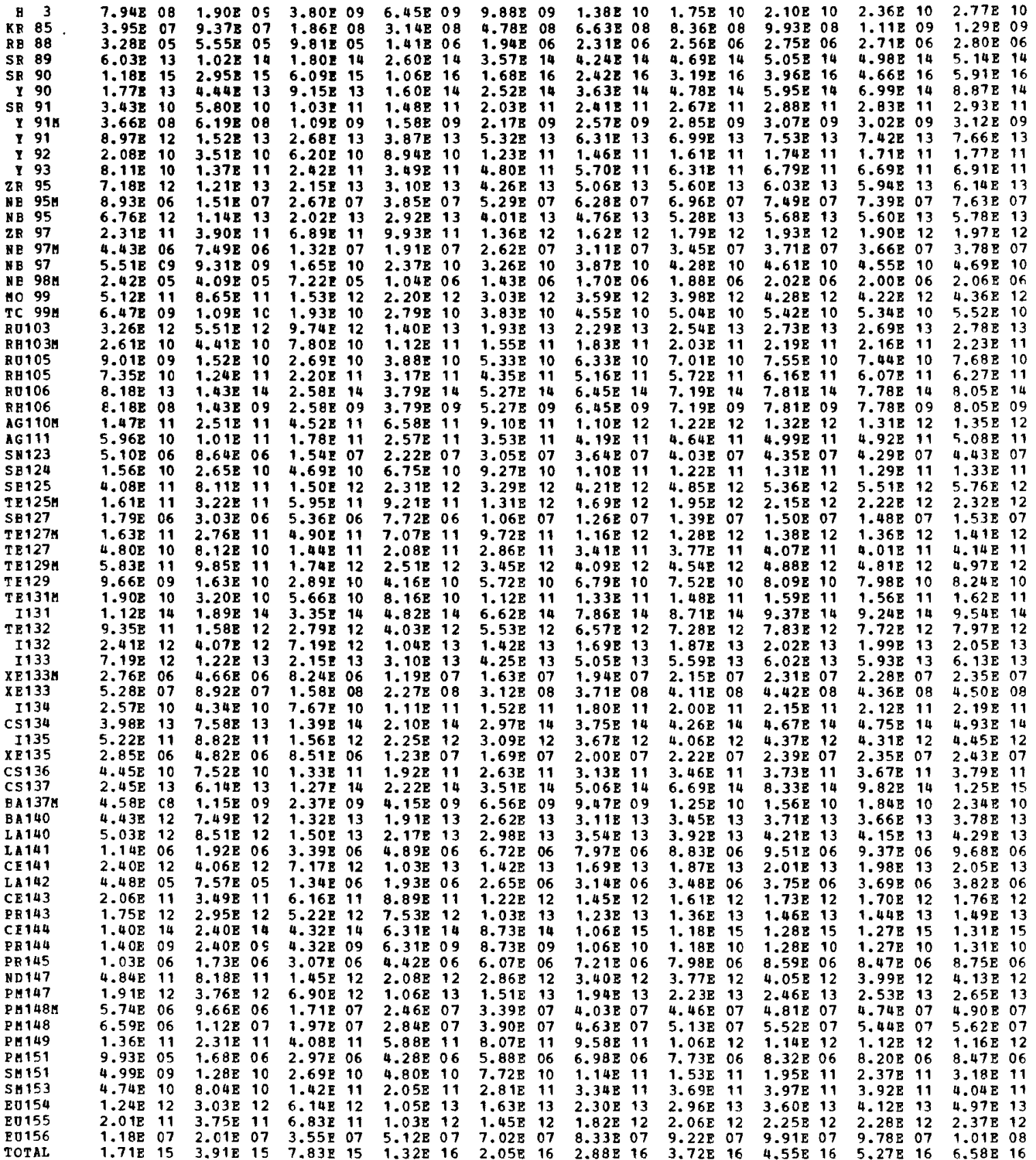


Table 3. 15. Ingesticn toxicity $\left(n^{3}\right.$ vater) of heary-elenent 1 sotopes accunulated in the 0 . $S$.

$\begin{array}{llllllllll}1980 & 1985 & 1990 & 1995 & 2000 & 2005 & 2010 & 2015 & 2020 & 2030\end{array}$

\begin{tabular}{|c|c|c|c|c|c|c|c|c|c|c|c|c|c|c|c|c|c|c|}
\hline $\begin{array}{l}1.92 \mathrm{E} \\
2.37 \mathrm{~B} \\
7.31 \mathrm{E} \\
5.83 \mathrm{E} \\
2.12 \mathrm{E} \\
2.19 \mathrm{E} \\
3.26 \mathrm{E} \\
5.29 \mathrm{E} \\
2.82 \mathrm{~B} \\
1.80 \mathrm{E} \\
9.13 \mathrm{~B} \\
7.95 \mathrm{E} \\
5.80 \mathrm{E} \\
2.58 \mathrm{E} \\
2.00 \mathrm{~B} \\
1.44 \mathrm{E} \\
1.20 \mathrm{E} \\
9.27 \mathrm{E} \\
1.15 \mathrm{E}\end{array}$ & $\begin{array}{l}08 \\
11 \\
08 \\
10 \\
12 \\
12 \\
11 \\
11 \\
12 \\
09 \\
08 \\
11 \\
09 \\
09 \\
10 \\
12 \\
10 \\
11 \\
13\end{array}$ & $\begin{array}{l}5.038 \\
4.02 \mathrm{E} \\
1.23 \mathrm{E} \\
9.92 \mathrm{E} \\
3.58 \mathrm{~B} \\
5.67 \mathrm{E} \\
8.45 \mathrm{E} \\
1.37 \mathrm{E} \\
6.81 \mathrm{~B} \\
4.67 \mathrm{E} \\
1.55 \mathrm{E} \\
2.90 \mathrm{E} \\
1.49 \mathrm{E} \\
4.53 \mathrm{E} \\
5.20 \mathrm{E} \\
2.42 \mathrm{E} \\
3.02 \mathrm{E} \\
2.28 \mathrm{E} \\
2.65 \mathrm{E}\end{array}$ & $\begin{array}{l}08 \\
11 \\
09 \\
10 \\
12 \\
12 \\
11 \\
12 \\
12 \\
09 \\
09 \\
12 \\
10 \\
09 \\
10 \\
12 \\
10 \\
12 \\
13\end{array}$ & 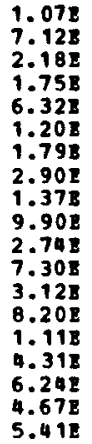 & $\begin{array}{l}09 \\
11 \\
09 \\
11 \\
12 \\
13 \\
12 \\
12 \\
13 \\
09 \\
09 \\
12 \\
10 \\
09 \\
11 \\
12 \\
10 \\
12 \\
13\end{array}$ & $\begin{array}{l}1.94 \mathrm{E} \\
1.03 \mathrm{E} \\
3.15 \mathrm{~B} \\
2.52 \mathrm{E} \\
9.12 \mathrm{~B} \\
2.138 \\
3.22 \mathrm{E} \\
5.23 \mathrm{E} \\
2.34 \mathrm{E} \\
1.78 \mathrm{E} \\
3.95 \mathrm{E} \\
1.52 \mathrm{E} \\
5.58 \mathrm{E} \\
1.23 \mathrm{E} \\
1.99 \mathrm{E} \\
6.25 \mathrm{E} \\
1.09 \mathrm{E} \\
8.05 \mathrm{~B} \\
9.35 \mathrm{E}\end{array}$ & $\begin{array}{l}09 \\
12 \\
09 \\
11 \\
12 \\
13 \\
12 \\
12 \\
13 \\
10 \\
09 \\
13 \\
10 \\
10 \\
11 \\
12 \\
11 \\
12 \\
13\end{array}$ & $\begin{array}{l}3.18 \mathrm{E} \\
1.41 \mathrm{~B} \\
4.32 \mathrm{E} \\
3.46 \mathrm{E} \\
1.25 \mathrm{~B} \\
3.44 \mathrm{E} \\
5.23 \mathrm{~B} \\
8.49 \mathrm{E} \\
3.62 \mathrm{~B} \\
2.90 \mathrm{~B} \\
5.42 \mathrm{E} \\
2.78 \mathrm{~B} \\
9.02 \mathrm{E} \\
1.74 \mathrm{E} \\
3.23 \mathrm{~B} \\
8.64 \mathrm{E} \\
1.73 \mathrm{E} \\
1.25 \mathrm{~B} \\
1.48 \mathrm{E}\end{array}$ & $\begin{array}{l}09 \\
12 \\
09 \\
11 \\
13 \\
13 \\
12 \\
12 \\
13 \\
10 \\
09 \\
13 \\
10 \\
10 \\
11 \\
12 \\
11 \\
13 \\
14\end{array}$ & $\begin{array}{l}4.76 \mathrm{E} \\
1.67 \mathrm{E} \\
5.13 \mathrm{E} \\
4.11 \mathrm{E} \\
1.49 \mathrm{E} \\
5.06 \mathrm{E} \\
7.78 \mathrm{E} \\
1.26 \mathrm{E} \\
5.09 \mathrm{E} \\
4.31 \mathrm{E} \\
6.43 \mathrm{E} \\
4.62 \mathrm{E} \\
1.33 \mathrm{E} \\
2.17 \mathrm{E} \\
4.81 \mathrm{E} \\
1.04 \mathrm{E} \\
2.50 \mathrm{E} \\
1.78 \mathrm{E} \\
2.14 \mathrm{E}\end{array}$ & $\begin{array}{l}09 \\
12 \\
09 \\
11 \\
13 \\
13 \\
12 \\
13 \\
13 \\
10 \\
09 \\
13 \\
11 \\
10 \\
11 \\
13 \\
11 \\
13 \\
14\end{array}$ & $\begin{array}{l}6.56 z \\
1.85 E \\
5.69 E \\
4.54 E \\
1.65 E \\
6.83 E \\
1.06 E \\
1.73 E \\
6.53 B \\
5.898 \\
7.11 E \\
7.03 E \\
1.81 E \\
2.55 E \\
6.57 E \\
1.15 E \\
3.31 E \\
2.30 E \\
2.86 E\end{array}$ & $\begin{array}{l}09 \\
12 \\
09 \\
11 \\
13 \\
13 \\
13 \\
13 \\
13 \\
10 \\
09 \\
13 \\
11 \\
10 \\
11 \\
13 \\
11 \\
13 \\
14\end{array}$ & $\begin{array}{l}8.57 \mathrm{E} \\
1.99 \mathrm{E} \\
6.12 \mathrm{E} \\
4.88 \mathrm{E} \\
1.78 \mathrm{E} \\
8.72 \mathrm{E} \\
1.38 \mathrm{~B} \\
2.24 \mathrm{E} \\
7.89 \mathrm{E} \\
7.62 \mathrm{E} \\
7.65 \mathrm{E} \\
1.00 \mathrm{E} \\
2.33 \mathrm{E} \\
2.89 \mathrm{E} \\
8.50 \mathrm{~B} \\
1.25 \mathrm{E} \\
4.14 \mathrm{E} \\
2.81 \mathrm{~B} \\
3.65 \mathrm{E}\end{array}$ & $\begin{array}{l}12 \\
09 \\
11 \\
13 \\
13 \\
13 \\
13 \\
13 \\
10 \\
09 \\
14 \\
11 \\
10 \\
11 \\
13 \\
11 \\
13 \\
14\end{array}$ & $\begin{array}{l}1.06 \mathrm{E} \\
1.96 \mathrm{~B} \\
6.03 \mathrm{E} \\
4.82 \mathrm{E} \\
1.75 \mathrm{E} \\
1.06 \mathrm{E} \\
1.69 \mathrm{E} \\
2.75 \mathrm{E} \\
8.99 \mathrm{~B} \\
9.37 \mathrm{~B} \\
7.55 \mathrm{~B} \\
1.34 \mathrm{~B} \\
2.84 \mathrm{~B} \\
3.07 \mathrm{E} \\
1.04 \mathrm{~B} \\
1.23 \mathrm{E} \\
4.89 \mathrm{~B} \\
3.23 \mathrm{E} \\
4.41 \mathrm{E}\end{array}$ & $\begin{array}{l}10 \\
12 \\
09 \\
11 \\
13 \\
14 \\
13 \\
13 \\
13 \\
10 \\
09 \\
14 \\
11 \\
10 \\
12 \\
13 \\
11 \\
13 \\
14\end{array}$ & $\begin{array}{l}3.8 \mathrm{E} \\
3.07 \mathrm{E} \\
1.30 \mathrm{E} \\
7.78 \mathrm{E} \\
2.14 \mathrm{E} \\
3.87 \mathrm{E} \\
3.55 \mathrm{E} \\
1.45 \mathrm{E} \\
1.27 \mathrm{E} \\
6.26 \mathrm{E} \\
3.94 \mathrm{E} \\
6.01 \mathrm{E}\end{array}$ \\
\hline
\end{tabular}


Table 3.16. Inhalation toxicity $\left(\mathrm{s}^{3}\right.$ air) of light-elenent isotopes accululated in the 0 . $S$.

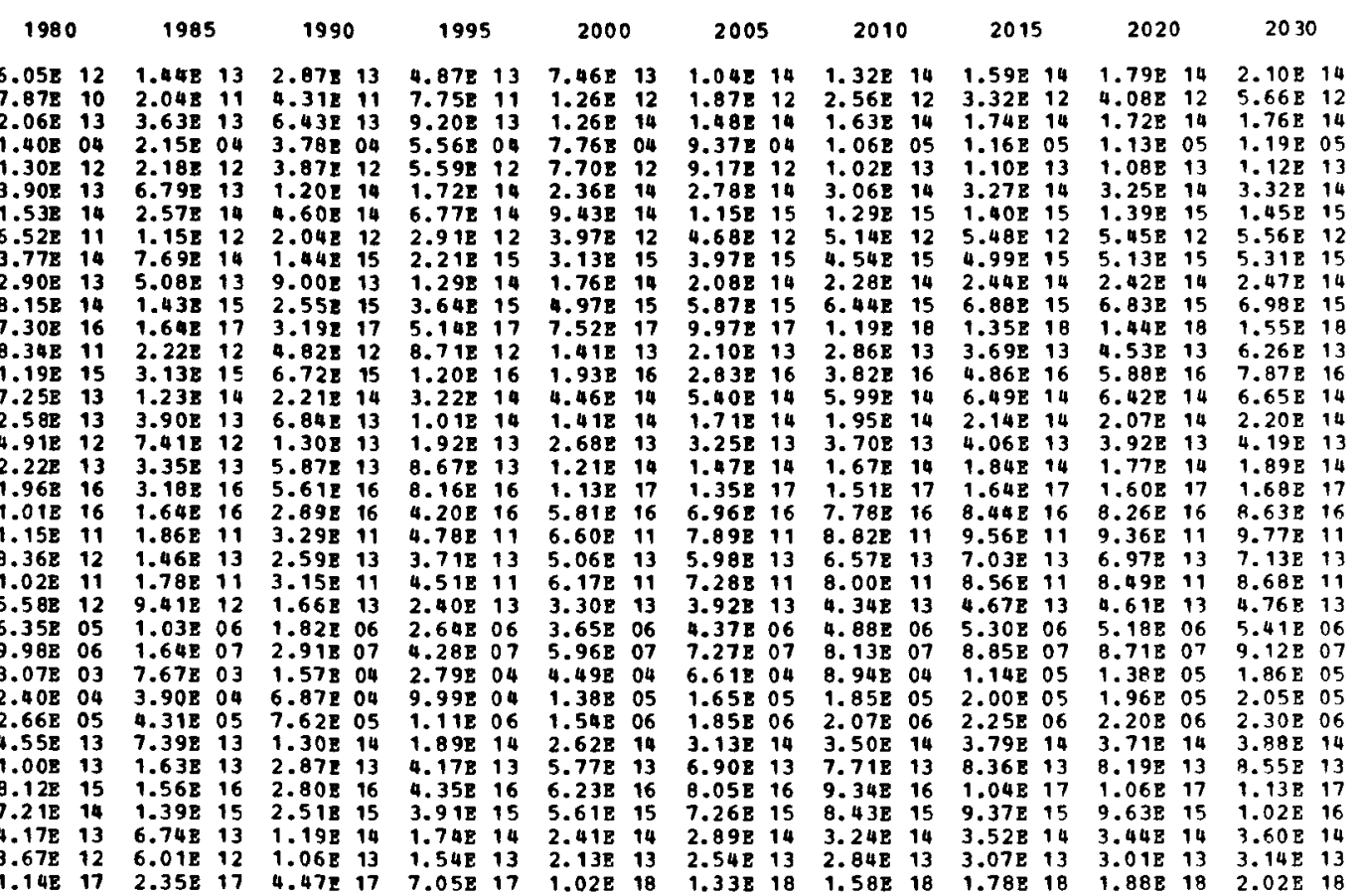


Table 3.17. Inhalaticn toxicity $\left(a^{3}\right.$ air) of fission-product isotopes accumalated in the 0 . $S$.

$\begin{array}{llllllllll}1980 & 1985 & 1990 & 1995 & 2000 & 2005 & 2010 & 2015 & 2020 & 2030\end{array}$

\begin{tabular}{|c|c|c|c|c|c|c|c|c|c|c|c|c|c|c|c|c|c|c|c|c|c|}
\hline H & 3 & $1.19 E$ & 13 & $2.85 E$ & 13 & & 13 & $.67 \mathrm{E}$ & & & 10 & 2.0 & & & & & & & & & \\
\hline $\mathrm{KR}$ & 85 & $1.32 \mathrm{E}$ & 14 & & 14 & & 14 & 0 5E & 15 & $1.59 \mathrm{E}$ & 15 & & 15 & & & & 15 & & DE & 4.298 & 15 \\
\hline $\mathrm{RE}$ & 88 & $3.28 \mathrm{E}$ & 05 & $5.55 E$ & 05 & $81 \bar{E}$ & 05 & & 06 & $1.94 \mathrm{E}$ & 06 & $31 \mathrm{E}$ & 06 & $56 E$ & 06 & $2.75 \mathrm{E}$ & 06 & $2.71 \mathrm{E}$ & 06 & $2.80 \mathrm{E}$ & 06 \\
\hline SR & 89 & $6.03 \mathrm{E}$ & 17 & $1.02 E$ & 18 & & 18 & $60 \mathrm{E}$ & 18 & 78 & 18 & $.24 \mathrm{E}$ & 18 & & 18 & $5.05 \mathrm{~B}$ & 18 & $.98 \mathrm{~B}$ & 18 & $5.14 \mathrm{E}$ & 18 \\
\hline SR & 90 & $1.18 \mathrm{BE}$ & 19 & $2.95 \mathrm{~B}$ & 19 & $6.09 \mathrm{E}$ & 19 & $06 \mathrm{E}$ & 20 & $1.68 \mathrm{E}$ & 20 & $.42 \mathrm{E}$ & 20 & 3.198 & 20 & $3.96 \mathrm{~B}$ & 20 & 4.668 & 20 & $5.91 \mathrm{~B}$ & 20 \\
\hline$y$ & 90 & $1.18 \mathrm{E}$ & 17 & $2.96 \mathrm{E}$ & 17 & & 17 & $.07 \mathrm{E}$ & 18 & $1.68 \mathrm{E}$ & 18 & $2.42 \mathrm{E}$ & 18 & 3.198 & 18 & & 8 & & 18 & $5.91 \mathrm{E}$ & 18 \\
\hline SR & 99 & $1.91 \mathrm{E}$ & 14 & $3.22 \mathrm{E}$ & 19 & .708 & 14 & $.22 \mathrm{E}$ & 14 & $1.13 \mathrm{E}$ & 15 & $1.34 \mathrm{E}$ & 15 & $1.48 \mathrm{E}$ & 15 & 608 & 15 & $1.57 \mathrm{~g}$ & 15 & $1.63 \mathrm{P}$ & 5 \\
\hline & $91 \mathrm{H}$ & & 12 & & 12 & & 12 & & & & & & 13 & & 13 & & & & 13 & $.56 \mathrm{E}$ & 13 \\
\hline$Y$ & 91 & $2.69 \mathrm{E}$ & 17 & $4.55 \mathrm{~B}$ & 17 & $8.05 \mathrm{E}$ & 17 & $1.16 \mathrm{E}$ & 18 & $1.60 \mathrm{E}$ & 18 & $1.89 \mathrm{E}$ & 18 & $2.10 \mathrm{E}$ & 18 & $2.26 \mathrm{E}$ & 18 & $2.23 \mathrm{E}$ & 18 & $2.30 \mathrm{E}$ & 18 \\
\hline Y & 92 & $25 E$ & 14 & $2.10 \mathrm{E}$ & 14 & $3.72 \mathrm{E}$ & 14 & $5.36 \mathrm{E}$ & 14 & & 14 & & 14 & $9.69 \mathrm{E}$ & 14 & $1.04 \mathrm{E}$ & 15 & $1.03 \mathrm{~B}$ & 15 & $.06 \mathrm{E}$ & \\
\hline 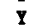 & 93 & $4.87 \mathrm{E}$ & 14 & $8.22 \mathrm{~B}$ & 14 & $1.45 \mathrm{E}$ & 15 & $2.10 \mathrm{E}$ & 15 & $2.88 \mathrm{E}$ & 15 & & 15 & & 15 & $4.07 \mathrm{E}$ & 15 & 4.028 & 15 & $4.15 \mathrm{E}$ & 15 \\
\hline ZR & 95 & $4.31 \mathrm{E}$ & 17 & $7.29 E$ & 17 & $.29 E$ & 18 & $1.86 \mathrm{E}$ & 18 & $2.55 \mathrm{E}$ & 18 & $3 E$ & 18 & $36 E$ & 18 & $3.62 E$ & 18 & $57 E$ & 18 & $3.68 E$ & 18 \\
\hline N B & 95: & $93 E$ & 06 & 1.518 & 07 & $.67 \mathrm{E}$ & 07 & $3.85 \mathrm{E}$ & 07 & & 07 & & 07 & & 07 & $7.49 \mathrm{E}$ & 07 & $39 \mathrm{~B}$ & 07 & $7.63 \mathrm{E}$ & 07 \\
\hline $\mathrm{ME}$ & 95 & $25 B$ & 17 & & 17 & $4 \bar{E}$ & 17 & $9.72 \mathrm{E}$ & 17 & $1.34 \mathrm{E}$ & 18 & & 18 & $76 \mathrm{E}$ & 18 & $1.89 \mathrm{E}$ & 18 & $1.87 \mathrm{E}$ & 18 & $1.93 \mathrm{E}$ & 18 \\
\hline $\mathbf{Z R}$ & 97 & $1.54 \mathrm{E}$ & 15 & $\mathrm{OE}$ & 15 & 598 & 15 & $6.62 \mathrm{E}$ & 15 & $9.10 \mathrm{E}$ & 15 & & 16 & & 16 & $1.29 \mathrm{E}$ & & & 16 & & 16 \\
\hline $\mathrm{NB}$ & $97 \mathrm{~m}$ & $4.43 \mathrm{~B}$ & 06 & $49 \mathrm{E}$ & 06 & $1.32 \mathrm{E}$ & 07 & $1.91 \mathrm{E}$ & 07 & $2.62 \mathrm{E}$ & 07 & $11 \mathrm{E}$ & 07 & $3.45 \mathrm{E}$ & 07 & 3.718 & 07 & $3.66 \mathrm{~B}$ & 07 & $3.78 \mathrm{E}$ & 07 \\
\hline & 97 & $2.48 E$ & 13 & & 13 & & 13 & & 14 & & 14 & & & & 14 & & & & 14 & $2.11 E$ & 14 \\
\hline & $98 \mathrm{H}$ & $2.42 \mathrm{E}$ & 05 & $4.09 \mathrm{E}$ & 05 & $7.22 \mathrm{E}$ & 05 & $1.04 E$ & 06 & $1.43 \mathrm{E}$ & 06 & $10 E$ & 06 & $88 \mathrm{E}$ & 06 & $02 E$ & 06 & $00 \mathrm{E}$ & 06 & $2.06 \mathrm{E}$ & 06 \\
\hline & 99 & $2.92 \mathrm{~B}$ & 15 & $4.94 \mathrm{E}$ & 15 & & 15 & $1.26 \mathrm{E}$ & 16 & & & & 16 & & 16 & & & & & & 16 \\
\hline & 994 & $3.88 \mathrm{~B}$ & 13 & $6.56 \mathrm{E}$ & 13 & $1.16 \mathrm{E}$ & 14 & $1.67 \mathrm{E}$ & 14 & $2.30 \mathrm{E}$ & 14 & & 14 & 028 & 14 & $3.25 \mathrm{E}$ & 14 & $3.21 \mathrm{E}$ & 14 & $3.31 \mathrm{E}$ & 14 \\
\hline RO & 1103 & $59 \mathrm{E}$ & 16 & $1.47 \mathrm{~B}$ & 17 & $60 \mathrm{~B}$ & 17 & $3.75 \mathrm{E}$ & 17 & $5.15 \mathrm{E}$ & 17 & & 17 & $6.77 \mathrm{E}$ & 17 & $7.28 \mathrm{E}$ & 17 & $7.18 \mathrm{E}$ & 17 & $7.41 \mathrm{R}$ & 17 \\
\hline 80 & $1103 \mathrm{H}$ & & 14 & $2.21 E$ & 14 & & 14 & $5.62 \mathrm{E}$ & 14 & $7.73 E$ & 14 & & 14 & $1.02 E$ & 15 & & 15 & & 15 & $1.11 \mathrm{E}$ & 15 \\
\hline RO & 105 & ;1E & 13 & $62 \mathrm{E}$ & 13 & $35 \mathrm{E}$ & 14 & $94 \mathrm{E}$ & 14 & $67 \mathrm{E}$ & 14 & & 14 & 518 & 14 & $77 \mathrm{E}$ & 14 & $.72 \mathrm{E}$ & 14 & $3.84 \mathrm{E}$ & 14 \\
\hline R日 & 1105 & $3.68 \mathrm{BE}$ & 14 & $6.21 \mathrm{E}$ & 14 & $1.10 \mathrm{E}$ & 15 & $1.58 \mathrm{E}$ & 15 & $2.18 \mathrm{E}$ & 15 & & 15 & $2.86 \mathrm{E}$ & 15 & $3.08 \mathrm{E}$ & 15 & & 15 & $3.13 \mathrm{E}$ & 15 \\
\hline RO & 1106 & $4.09 \mathrm{E}$ & 18 & 7.138 & $1 \varepsilon$ & 1.298 & 19 & $1.90 \mathrm{E}$ & 19 & $2.63 \mathrm{E}$ & 19 & & 19 & $3.60 \mathrm{E}$ & 19 & $3.90 \mathrm{~B}$ & 9 & $89 \mathrm{~B}$ & 19 & $4.02 \mathrm{E}$ & \\
\hline RB & 3906 & $8.18 \mathrm{E}$ & 08 & $1.43 \mathrm{E}$ & 09 & $58 E$ & 09 & $3.79 \mathrm{E}$ & 09 & $5.27 \mathrm{E}$ & 09 & & 09 & 7.198 & 09 & $1 \mathrm{E}$ & 09 & $78 \mathrm{E}$ & 09 & 158 & 09 \\
\hline AG & & & 16 & $2.51 \mathrm{~B}$ & $1 \epsilon$ & & 16 & $.58 \mathrm{E}$ & 16 & $9.10 \mathrm{E}$ & & & 17 & $1.22 E$ & 17 & & & 318 & 17 & $35 \mathrm{E}$ & 17 \\
\hline AG & $\$ 111$ & $2.98 \mathrm{E}$ & 14 & $5.04 \mathrm{E}$ & 14 & $1 E$ & 14 & $1.28 \mathrm{E}$ & 15 & $1.76 \mathrm{E}$ & $15^{\circ}$ & & 15 & $2.32 \mathrm{E}$ & 15 & $50 \mathrm{E}$ & 15 & $2.46 \mathrm{E}$ & 15 & $2.54 \mathrm{E}$ & 15 \\
\hline SN & 1123 & $1 O E$ & 06 & $8.64 \mathrm{E}$ & 06 & & 07 & $2.22 \mathrm{E}$ & 07 & $3.05 \mathrm{E}$ & 07 & & 07 & $4.03 E$ & 07 & & 7 & $29 E$ & 07 & $4.43 \mathrm{E}$ & 07 \\
\hline $\mathrm{SE}$ & 124 & $4.45 \mathrm{E}$ & 14 & $7 \mathrm{E}$ & 14 & $34 \mathrm{E}$ & 15 & $1.93 \mathrm{E}$ & 15 & $2.65 \mathrm{E}$ & 15 & & 15 & $3.48 \mathrm{E}$ & 15 & 3.748 & 15 & $69 \mathrm{E}$ & 15 & $3.80 \mathrm{E}$ & 15 \\
\hline $\mathrm{SE}$ & 125 & 38 & 16 & & 16 & & 17 & $2.56 \mathrm{E}$ & 17 & $3.65 \mathrm{E}$ & 17 & & 17 & $39 E$ & 17 & & 17 & & 17 & $6.40 \mathrm{E}$ & 17 \\
\hline TE & 125 & $4.02 \mathrm{E}$ & 15 & $8.05 \mathrm{E}$ & 15 & 1. & 16 & $2.30 \mathrm{E}$ & 16 & $3.28 \mathrm{E}$ & 16 & & 16 & 4. $87 \mathrm{E}$ & 16 & & 16 & $5.54 \mathrm{~B}$ & 16 & $.80 \mathrm{E}$ & 16 \\
\hline SE & 127 & $1.79 \mathrm{E}$ & 06 & $3.03 \overline{8}$ & 06 & & 06 & $7.72 \mathrm{E}$ & 06 & $1.06 \mathrm{E}$ & 07 & & 07 & $1.39 \mathrm{E}$ & 07 & & 0 & $48 \mathrm{E}$ & 07 & $3 E$ & 07 \\
\hline TE & $127 \mathrm{~m}$ & 8.15E & 15 & $1.38 \mathrm{E}$ & 16 & & 16 & $3.53 \mathrm{E}$ & 16 & $4.86 \mathrm{E}$ & 16 & & 16 & $6.42 \mathrm{E}$ & 16 & & 16 & 82E & & $7.04 \mathrm{E}$ & 16 \\
\hline & & & 14 & & 14 & & 14 & & 15 & & 15 & & 1 & & 15 & & & & & $6 \mathrm{E}$ & 15 \\
\hline TE & 1294 & $1.17 \mathrm{E}$ & 16 & $1.97 \mathrm{E}$ & 16 & $3.48 \mathrm{~B}$ & 16 & $5.02 \mathrm{E}$ & 16 & $6.90 \mathrm{E}$ & 16 & & 16 & $07 \mathrm{E}$ & 16 & $\mathrm{E}$ & & $62 \mathrm{~B}$ & 16 & $94 \mathrm{E}$ & 16 \\
\hline TE & 2129 & $7.73 \mathrm{E}$ & 13 & $1.31 \mathrm{E}$ & 14 & $2.31 \mathrm{E}$ & 14 & $3.33 \mathrm{E}$ & 14 & $4.58 \mathrm{E}$ & 14 & & 14 & $6.02 \mathrm{E}$ & 14 & & 1 & & 1 & $59 \mathrm{E}$ & 14 \\
\hline TF & & & 14 & & 14 & & 14 & $44 \mathrm{E}$ & 14 & & 14 & & 14 & & 14 & & 1 & & 1 & & 15 \\
\hline$I$ & 139 & $3.36 \mathrm{E}$ & 17 & $5.68 \mathrm{E}$ & 17 & $1.00 \mathrm{E}$ & 18 & $1.45 \mathrm{E}$ & 18 & $1.99 \mathrm{E}$ & 18 & & 18 & $2.61 \mathrm{E}$ & 18 & $81 \mathrm{E}$ & 18 & $77 \mathrm{E}$ & 18 & $2.86 \mathrm{E}$ & 18 \\
\hline$T E$ & 132 & $68 \mathrm{~B}$ & 15 & & 15 & & 16 & $2.01 \mathrm{E}$ & 16 & $2.77 \mathrm{~B}$ & 16 & & 16 & & 16 & & & & 1 & & 16 \\
\hline$I$ & 1132 & $6.42 \mathrm{~B}$ & 15 & $1.09 \mathrm{E}$ & 16 & $1.92 \mathrm{E}$ & 16 & 2.778 & 16 & $3.80 \mathrm{E}$ & 16 & & 16 & $4.99 \mathrm{E}$ & 16 & 388 & 6 & $30 \mathrm{~B}$ & 16 & $5.47 \mathrm{~B}$ & 16 \\
\hline$I$ & 1933 & $1.80 \mathrm{E}$ & 16 & $3.04 \mathrm{E}$ & 16 & $5.37 \mathrm{E}$ & 16 & 7.748 & 16 & $1.06 \mathrm{E}$ & 17 & $26 \mathrm{E}$ & 17 & $1.40 \mathrm{E}$ & 17 & $51 \mathrm{E}$ & 17 & $48 \mathrm{~B}$ & 17 & $1.53 \mathrm{E}$ & 17 \\
\hline$x E$ & 2133 & & 12 & & 13 & & 13 & $3.96 \mathrm{E}$ & 13 & & 13 & & 13 & & 13 & & & & 1 & & 13 \\
\hline$X E$ & 133 & & 14 & & 14 & & 14 & $7.58 \mathrm{E}$ & 14 & 1.0 & 15 & & 15 & & 15 & & & & 1 & $0 \mathrm{E}$ & 15 \\
\hline & {$[134$} & & 13 & & in & & 14 & & 14 & & 14 & & 1 & & 14 & & & & & & 14 \\
\hline $\mathrm{CS}$ & 5134 & $8.94 \mathrm{E}$ & 17 & $9.71 \mathrm{E}$ & 18 & $12 \mathrm{E}$ & 18 & $4.74 \mathrm{E}$ & 18 & $6.68 \mathrm{E}$ & 18 & $43 \mathrm{E}$ & 18 & $9.58 \mathrm{E}$ & 18 & $.05 \mathrm{~B}$ & 19 & $07 \mathrm{~B}$ & 19 & $1.11 \mathrm{E}$ & 19 \\
\hline & & & 15 & & 15 & & 15 & & 15 & & 16 & & 16 & & 16 & & 1 & & 16 & $8 \bar{E}$ & 16 \\
\hline$X \bar{E}$ & 135 & $2.85 \mathrm{~B}$ & 13 & & 13 & $1 E$ & 13 & $1.23 E$ & 14 & $1.69 \mathrm{E}$ & 14 & & 1 & & 14 & & & & & $3 \mathrm{E}$ & 14 \\
\hline $\operatorname{cs}$ & $\$ 136$ & & 14 & & 14 & & 15 & $92 \mathrm{E}$ & 15 & & 15 & & 1 & $3.46 \mathrm{E}$ & 15 & & 15 & & 5 & & 15 \\
\hline CS & $\$ 137$ & & 17 & & 18 & & 18 & $8.8 \mathrm{BE}$ & 18 & 1.4 & 19 & & 1 & $2.67 \mathrm{E}$ & 19 & & & & 19 & $1 \mathrm{E}$ & 19 \\
\hline BI & 137 & & $\mathrm{CB}$ & & OS & & 09 & $4.15 E$ & 09 & & 09 & & 0 & $25 \mathrm{E}$ & 10 & & 10 & & 10 & $34 \mathrm{E}$ & 10 \\
\hline $\mathrm{EA}$ & 1140 & $8.86 \mathrm{~B}$ & 16 & & 17 & & 17 & $3.82 \mathrm{E}$ & 17 & & 17 & & 17 & & 17 & & 1 & & 17 & & 17 \\
\hline & & & 16 & & 16 & & 16 & 1.0 & 17 & & 17 & & 17 & & 17 & & i & & 17 & & 17 \\
\hline & & & 06 & & 06 & & 06 & & 06 & & 06 & & or & & 06 & & & & 0 & & 06 \\
\hline CE & 141 & $4.32 \mathrm{E}$ & 16 & E & 16 & & 17 & $1.86 \mathrm{E}$ & 17 & $2.56 \mathrm{E}$ & 17 & & 17 & & 17 & & & & & & 17 \\
\hline IA & 142 & $4.48 \mathrm{E}$ & 05 & & 05 & & 06 & $1.93 \mathrm{E}$ & 06 & & 06 & & 06 & $3.48 \mathrm{E}$ & 06 & & 06 & & 06 & & 06 \\
\hline CE & 143 & & 15 & $1.99 \mathrm{E}$ & 15 & & 15 & $5.08 \mathrm{E}$ & 15 & $6.97 \mathrm{E}$ & 15 & & & $9.17 \mathrm{E}$ & 15 & & & & & & 16 \\
\hline PR & 1943 & 1.468 & 16 & 168 & 16 & & 16 & $6.27 E$ & 16 & & 16 & & 17 & & 17 & & 17 & & 17 & & 17 \\
\hline CE & & $6.98 \mathrm{E}$ & 18 & & 19 & & 19 & $3.15 E$ & 19 & & 19 & & 19 & & 19 & & 1 & & 19 & & 19 \\
\hline PE & & & 09 & & 09 & & 09 & & 09 & & 09 & & 1 & & 10 & & 1 & & 10 & & 10 \\
\hline PR & 145 & $1.03 \mathrm{~B}$ & 06 & & 06 & & 06 & & 06 & & 06 & & 06 & & 06 & & 0 & & 06 & & 06 \\
\hline NI & & & 15 & & 15 & & 16 & $1.56 \mathrm{E}$ & 16 & & 16 & & 16 & & 16 & & 1 & & 16 & & 16 \\
\hline & & & 17 & & 17 & & 17 & & 18 & & 18 & & & & 18 & & & & & & 18 \\
\hline P & 1148 & 5. & 06 & $9.66 \mathrm{E}$ & 06 & & 07 & $2.46 \mathrm{~B}$ & 07 & $3.39 \mathrm{E}$ & 07 & & 07 & $5 \mathrm{~B}$ & 07 & & & & 07 & $4.90 \mathrm{E}$ & 07 \\
\hline P N & $14 \mathrm{c}$ & & 06 & & 07 & $97 \mathrm{E}$ & 07 & $2.84 \mathrm{E}$ & 07 & 3.9 & 07 & & 07 & $5.13 E$ & 07 & & 0 & & 07 & $5.62 E$ & 07 \\
\hline P I & & & 14 & & 15 & & 15 & 2.948 & 15 & & & & & & 15 & & & & & & 15 \\
\hline P : & 151 & & 05 & & 06 & $2.97 \mathrm{E}$ & 06 & $4.28 \mathrm{E}$ & 06 & 5.8 & 06 & & 06 & $7.73 \mathrm{E}$ & 06 & & 5 & & 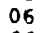 & $8.47 \mathrm{E}$ & 06 \\
\hline & & & 14 & & & & & & & & & & & & 16 & & & & & & 16 \\
\hline SE & 153 & 98 & 14 & 6.438 & 14 & 1.1 & 15 & $1.64 \mathrm{E}$ & 15 & $2.25 \mathrm{~B}$ & 15 & & 15 & $2.96 \mathrm{E}$ & 15 & $8 \mathrm{~B}$ & 5 & $13 E$ & 15 & $3.23 \mathrm{E}$ & 15 \\
\hline & 154 & & 17 & & 17 & & & $2.11 \mathrm{E}$ & 18 & & 18 & & & & 18 & & & & & & 18 \\
\hline & & & 16 & & 16 & & 16 & $6.87 E$ & 16 & & 16 & & 17 & 1. 378 & 17 & & & $52 E$ & 17 & $1.58 \mathrm{E}$ & 17 \\
\hline E & 1156 & & 07 & $2.01 \mathrm{E}$ & 07 & & 07 & $5.12 \mathrm{E}$ & 07 & & 07 & & 0 & $9.22 \mathrm{E}$ & 07 & & & $78 \mathrm{E}$ & 07 & $1.01 \mathrm{~B}$ & \\
\hline TO? & JTAL & $2.76 \mathrm{E}$ & 19 & $5.79 \mathrm{~B}$ & & $1.13 \mathrm{E}$ & & $1.85 E$ & 20 & $2.79 \mathrm{E}$ & 20 & & & $4.79 E$ & 20 & $5.76 \mathrm{E}$ & & $6.52 \mathrm{E}$ & 20 & $7.96 \mathrm{E}$ & 20 \\
\hline
\end{tabular}


Table 3.18. Inhalation toxicity $\left(\mathrm{e}^{3}\right.$ air) of heary-elenent 1 sotopes accueviated in the 0 . $S$.

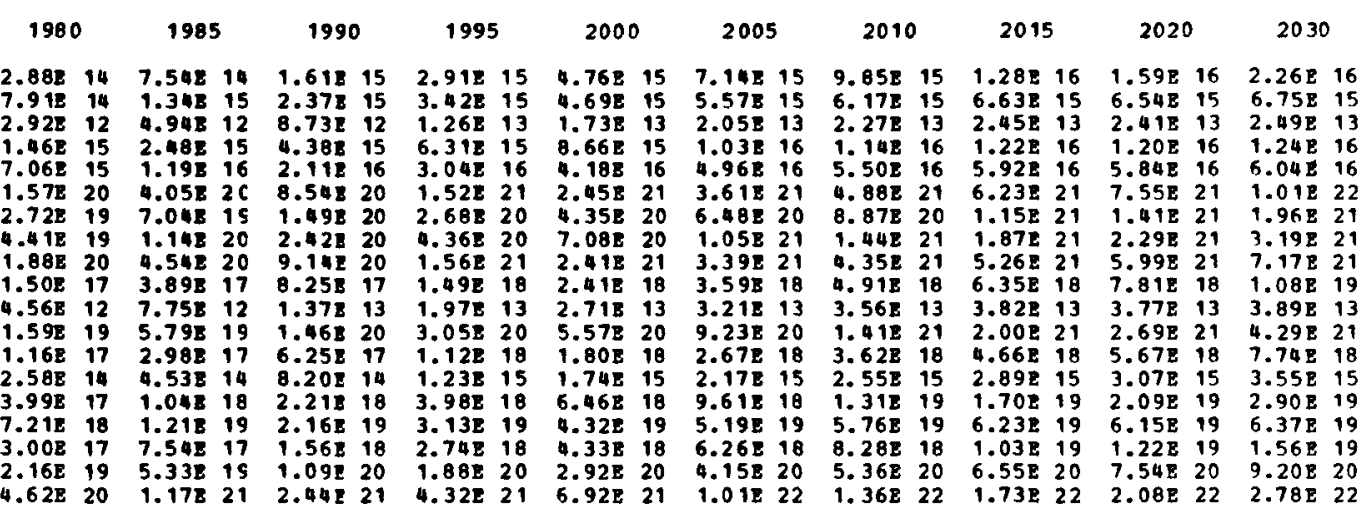


Table 3.19. Grans of accunulated 11ght elenents after 2031

\begin{tabular}{|c|c|}
\hline & 100 \\
\hline
\end{tabular}

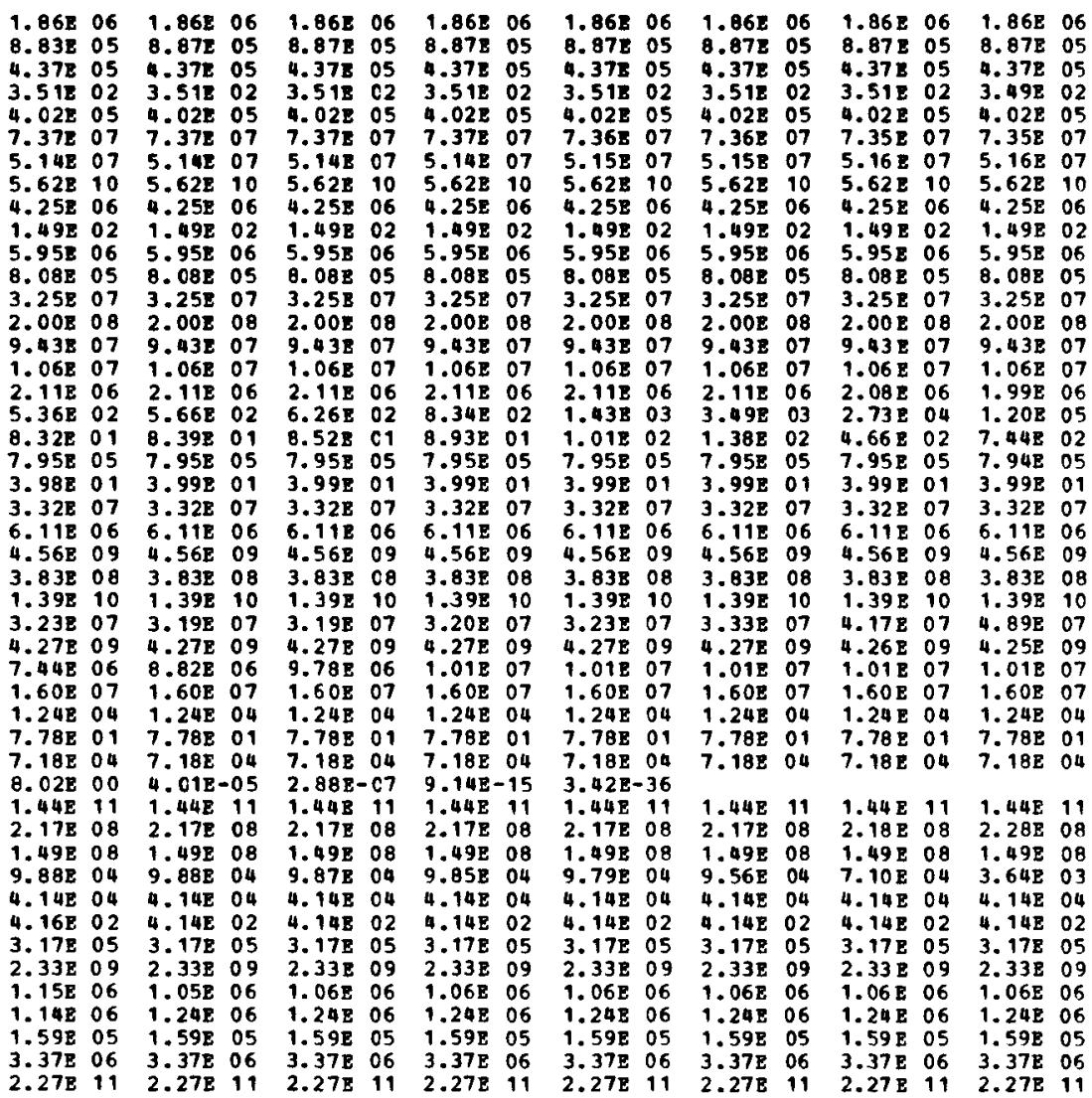


Table 3.20. Grans of accuanlated fission-product elements after 2031

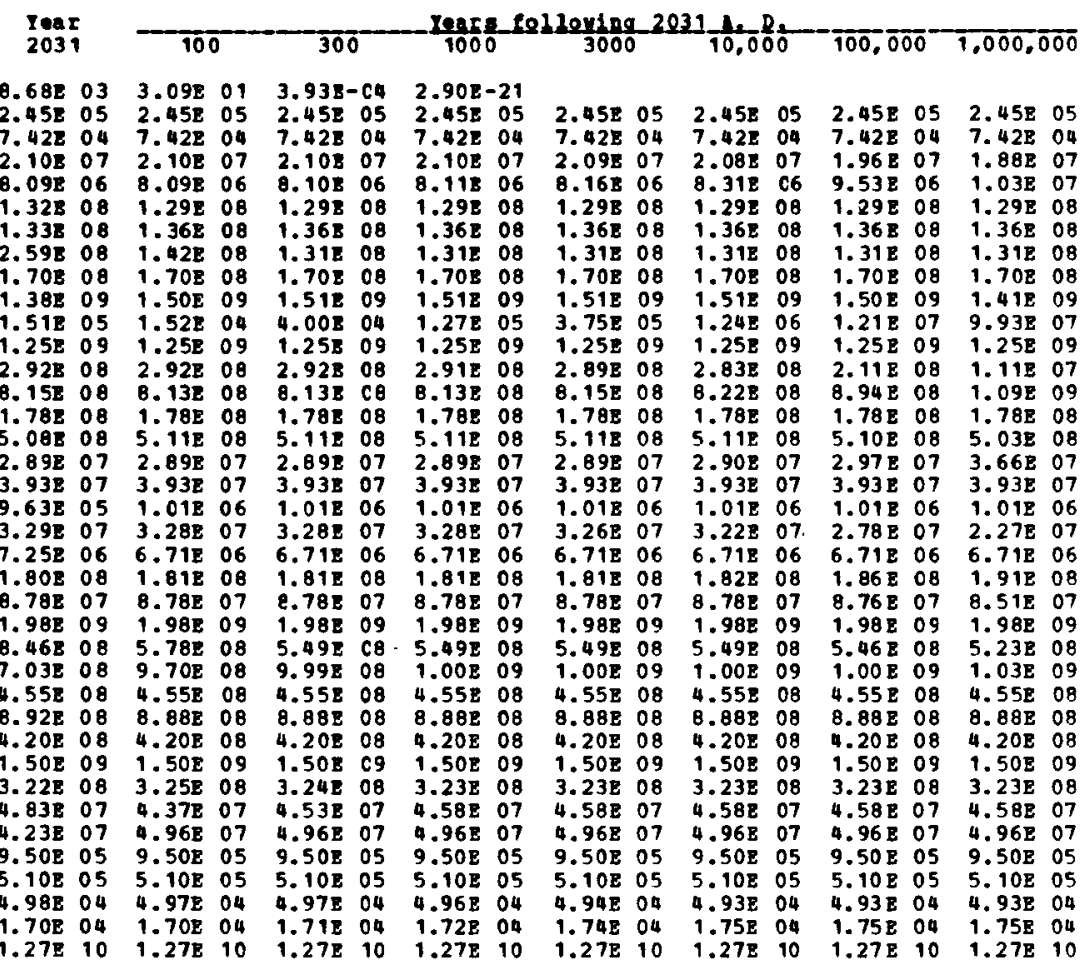

Table 3.21. Graus of accunulated heavy elenents after 2031

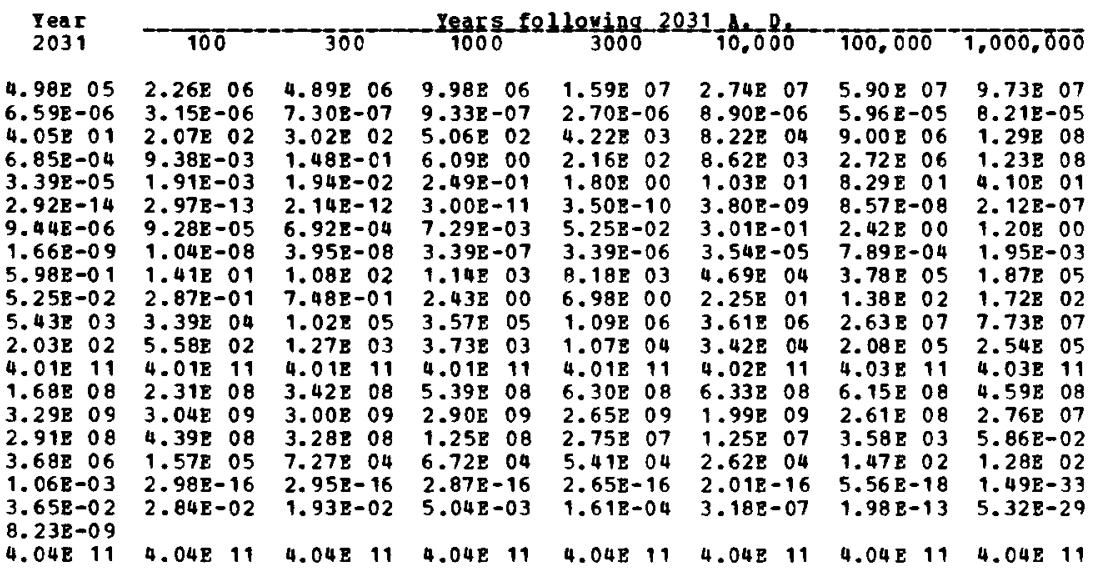


Iable 3.22. Curles of accunulated $11 \mathrm{ght}$-elenent isotopes after 2031

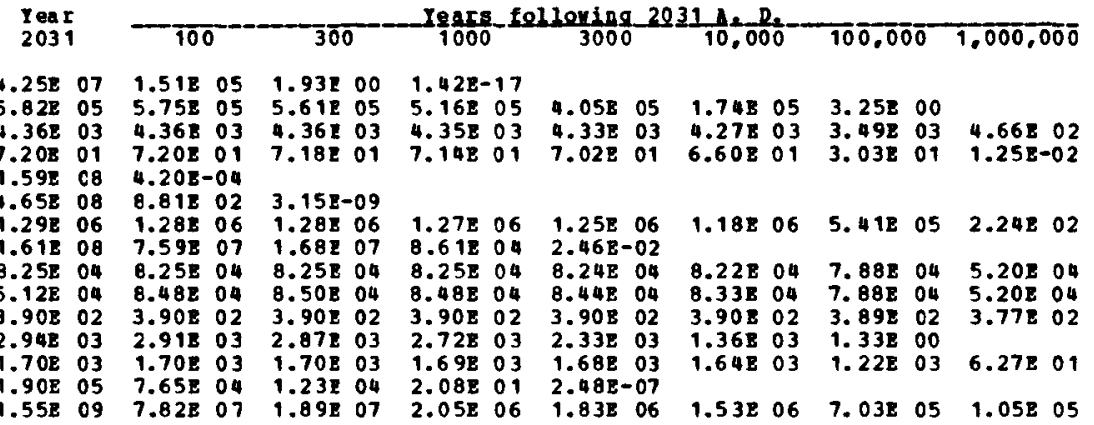

Table 3.23. Curies of accunulated fission-product isotopes after 2031

Yea $\mathbf{r}$

2031

$8.41 \mathrm{E} 07$

$\begin{array}{ll}1.52 \mathrm{E} & 05 \\ 1.30 \mathrm{E} & 09\end{array}$

$\begin{array}{ll}1.30 \mathrm{E} & 09 \\ 1.80 \mathrm{E} & 10\end{array}$

$\begin{array}{ll}1.81 \mathrm{E} & 10 \\ 6.88 \mathrm{E} & 05\end{array}$

$\begin{array}{rl}4.198 & 05 \\ 4.988 & 06\end{array}$

$\begin{array}{ll}4.98 E & 06 \\ 3.918 & 04\end{array}$

$2.87 \mathrm{E}$ O5

$\begin{array}{ll}2.885 & 05 \\ 7.87 E & 05\end{array}$

1.098 04

$1.11 \mathrm{E}$ 05

$2.55 \mathrm{E} \quad 10$

$2.38 \mathrm{E} 10$

$1.30 \mathrm{E} 08$

$\begin{array}{rl}1.01 \mathrm{E} & 09 \\ \mathrm{1} .61 \mathrm{E} & 02\end{array}$

$\begin{array}{ll}8.61 \mathrm{E} & 02 \\ 1.66 \mathrm{E} & 11\end{array}$

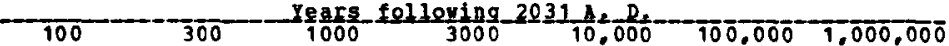

2.99E $05 \quad 3.81 E 00 \quad 2.81 E-17$

$\begin{array}{llllllllllllll}1.52 \mathrm{E} & 05 & 1.52 \mathrm{E} & 05 & 1.51 \mathrm{E} & 05 & 1.48 \mathrm{E} & 05 & 1.37 \mathrm{E} & 05 & 5.25 \mathrm{E} & 04 & 3.56 \mathrm{E} & 00\end{array}$ $12 E$ of $5.65 E$ DO $1.748-19$ $1.53 E$ OS $1.10 E$ OT $3.49 \mathrm{E}-01$

$\begin{array}{llllll}.53 E & 0 S & 1.10 E & 07 & 3.49 \mathrm{E}-01 & 1.31 \mathrm{E}-22 \\ .53 \mathrm{E} & 0 \mathrm{~S} & 1.10 \mathrm{E} & 07 & 3.49 \mathrm{~B}-01 & 1.31 \mathrm{E}-22\end{array}$

$\begin{array}{llllllllllll} & & & \end{array}$

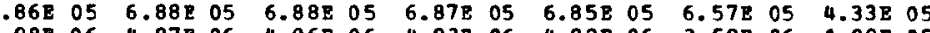

$\begin{array}{llllllllll} & \end{array}$

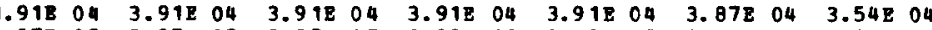

$\begin{array}{llllllllllllll}2.87 \mathrm{~B} & 05 & 2.87 \mathrm{E} & 05 & 2.85 \mathrm{E} & 05 & 2.82 \mathrm{E} & 05 & 2.68 \mathrm{E} & 05 & 1.44 \mathrm{E} & 05 & 2.81 \mathrm{E} & 02\end{array}$

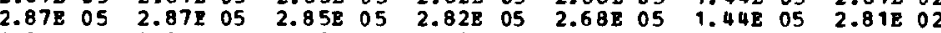

$\begin{array}{llllllllllllllll}2.84 E & 05 & 2.84 E & 05 & 2.83 E & 05 & 2.79 E & 05 & 2.66 E & 05 & 1.42 E & 05 & 2.78 E & 02\end{array}$

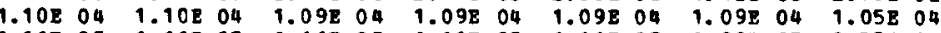

$1.11 \mathrm{E} 05$ 1.11E 05 1.11E 05 1.11E 05 1.11E 05 $1.09 \mathrm{E}$ 05 8.85E O4

$53 \mathrm{E}$ OS $2.49 \mathrm{E} 07 \quad 2.36 \mathrm{E} 00 \quad 2.01 \mathrm{E}-20$

$2.37 \mathrm{~B}$ OS $2.33 \mathrm{~B}$ 07 $2.20 \mathrm{~B}$ 0O $1.88 \mathrm{E}-20$

$5.87 \mathrm{E} 07$ 1.19E $07 \quad 4.54 \mathrm{E} 04 \quad 5.45 \mathrm{E}-03$

$1.32 \mathrm{E} 07 \quad 2.28 \mathrm{E} \quad 03 \quad 1.54 \mathrm{E}-10$

$\begin{array}{lllllllllll}8.13 \mathrm{E} & 02 & 7.24 \mathrm{E} & 02 & 4.83 \mathrm{E} & 02 & 1.52 \mathrm{E} & 02 & 2.67 \mathrm{~B} & 00 & 7.06 \mathrm{E}-23\end{array}$

$\begin{array}{llllllll}1.52 \mathrm{E} & 02 & 2.67 \mathrm{E} & 00 & 7.06 \mathrm{E}-23 & & \\ 7.45 \mathrm{E} & 06 & 7.29 \mathrm{E} & 06 & 5.54 \mathrm{E} & 06 & 1.19 \mathrm{E} & 06\end{array}$ 
Table 3.24. Curies of accunulated heavy-elenent isotopes after 2031

rear 2031 $100-\overline{3} 0 \overline{0}$

$3.82 \mathrm{E} 00$ 1. $04 \mathrm{E}-03$

$1.74 E-02$
$1.62 E-01$

$3.83 E 00$

5.59E-01

$1.62 E-0$

$3.83 \mathrm{E}$ 00

$4.74 \mathrm{E}-02$
$5.59 \mathrm{E}-\mathrm{CI}$

$5.598-C 1$
$1.53 E-01$

$4.64 \mathrm{E}-02$

5.59E-01

3.83E 00

$5.59 E-01$

$4.74 E-02$

$5.59 E-01$

$4.74 \mathrm{E}-02$

$4.738-02$

$5.598-01$

4.

.74E-02

$3.83 \mathrm{E}$ 00

$3.78 \mathrm{E}-00$
$4.72 \mathrm{E}-02$

$4.72 E-02$
8.748 OI

8.748
7.928 03

1.32805

$9.40 \mathrm{E} 00$

1.19805

1.77E 01

$4.65 E \quad 05$

$9.89 \mathrm{E} 03$

2.03E 08

$1.32 \mathrm{E}$ 05

1.19E 05

$1.82 E$
$7.28 E$ 08

$7.28 E$
1.218

1.21E 08

1.97E 08

$6.69 \mathrm{E}$ O5

$8.92 \mathrm{E} 08$

1.59E 06

$3.59 \mathrm{E} 06$

$2.56 \mathrm{E} 08$

$3.19 \mathrm{E} \quad 06$

2.80E 08

$2.63 E 10$

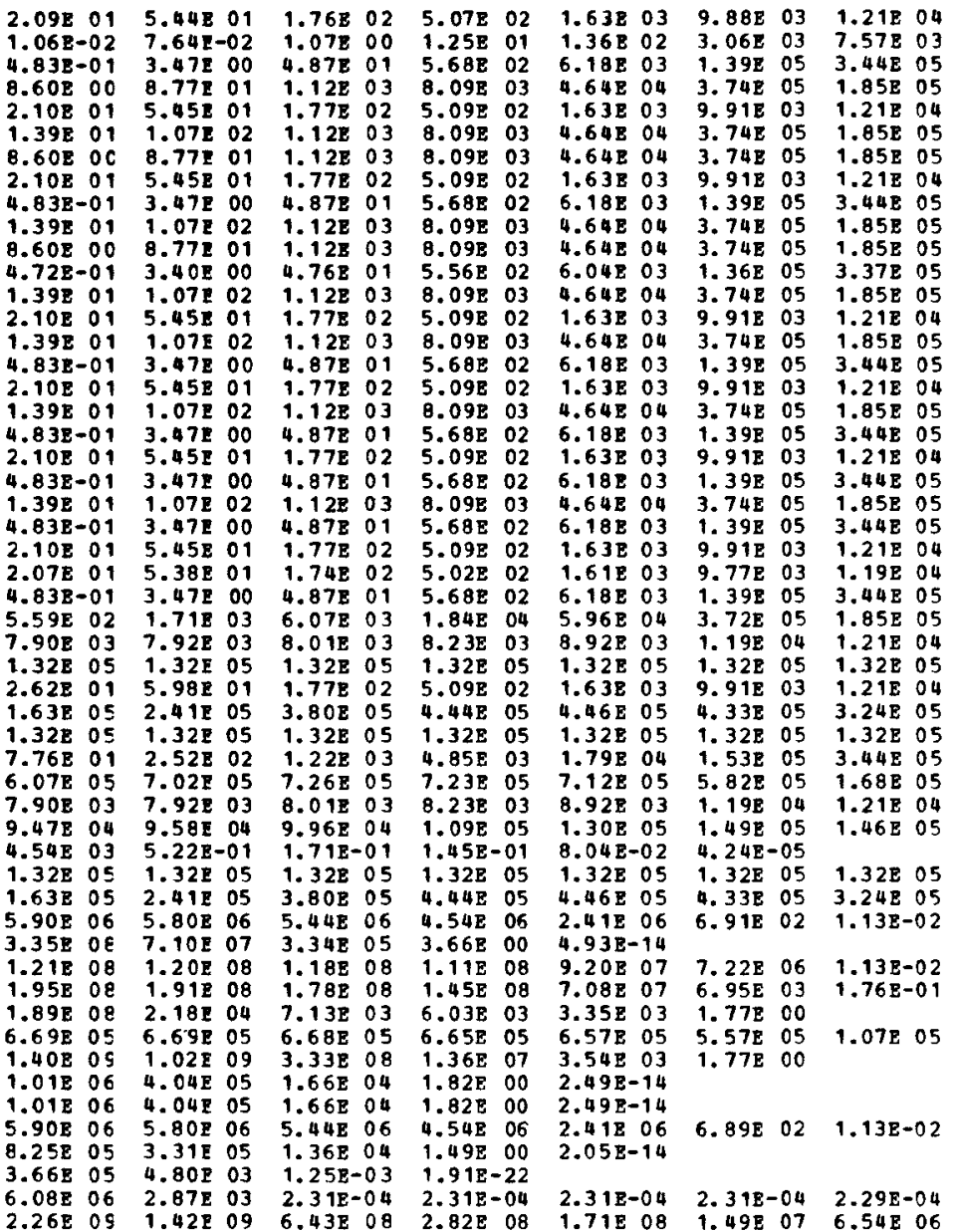


rable 3.25. Profectod accunulation of spent fool assonblies at sonrs (shipped 5 years after discharged)

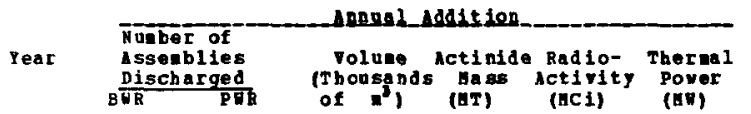

1985

$1985 \quad 5006 \quad 2373$

1987
1988

1988
1989

1990
1991

1992

1994

1995
1996

1997
1998

1999

2000

2002
2003

2004

2005

2007

2008

2009

2011
2012

2012

2014
2015

2015
2016
2017
2018

2019

2021

2022

2024
2025

2026

2028

2029

$2031 \quad 23692 \quad 1589$

$2032 \quad 23313 \quad 15236$

$2033 \quad 23196 \quad 15094$

$\begin{array}{lll}2034 & 23324 & 15088 \\ 2035 & 23489 & 15180\end{array}$

$$
\text { TIME APTER SHUT DOWh, YEARS }
$$
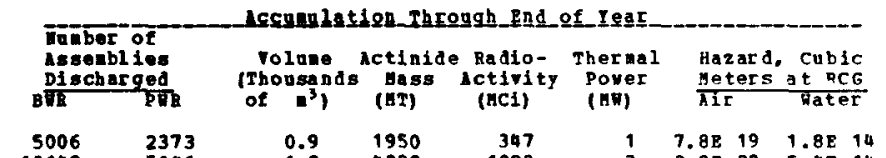

$\begin{array}{rrrr}0.87 & 1950 & 347 & 1 \\ 0.96 & 2150 & 696 & 2 \\ 1.04 & 2350 & 875 & 3 \\ 1.13 & 2550 & 1035 & 3 \\ 1.21 & 2749 & 1159 & 4 \\ 1.30 & 2949 & 1290 & 4\end{array}$

$\begin{array}{ll}5006 & 2373 \\ 10149 & 5141\end{array}$

$\begin{array}{rr}15199 & 8395 \\ 20598 & 11958\end{array}$

$\begin{array}{ll}20598 & 11958 \\ 26215 & 15881\end{array}$

$\begin{array}{ll}31926 & 20215 \\ 37944 & 24876\end{array}$

$\begin{array}{ll}37944 & 24876 \\ 44331 & 29839 \\ 51130 & 35087\end{array}$

$\begin{array}{ll}51130 & 35087 \\ 58356 & 40613\end{array}$

$\begin{array}{ll}58356 & 40613 \\ 65909 & 46332\end{array}$

$\begin{array}{ll}74105 & 52466 \\ 82978 & 59107\end{array}$

82978
92557
66217

$\begin{array}{ll}102862 & 73826 \\ 113938 & 81964\end{array}$

$\begin{array}{ll}113938 & 81964 \\ 125854 & 90673\end{array}$

$\begin{array}{rr}125854 & 90673 \\ 138627 & 99965\end{array}$

$166710 \quad 120268$

$182015 \quad 131253$

$198093 \quad 142748$

$231878 \quad 166786$

$249422 \quad 179216$

$267287 \quad 191865$

$285525 \quad 204814$

$304130 \quad 2180005$

$\begin{array}{ll}323090 & 231445 \\ 342764 & 245091\end{array}$

363227258904

383965
272970

$\begin{array}{ll}405242 & 287302 \\ 426201 & 302036\end{array}$

$447794 \quad 317007$

$\begin{array}{ll}470468 & 331848 \\ 492245 & 346593 \\ 513213 & 361666\end{array}$

$533956 \quad 376763$

$555233 \quad 391471$

$576958 \quad 406194$

$598610 \quad 421043$

$620547 \quad 436371$

$666144 \quad 467754$

$689988 \quad 483646$

$713679 \quad 499275$

$736992 \quad 514511$

$760188 \quad 529605$

$\begin{array}{ll}783511 & 544693 \\ 807000 & 559872\end{array}$

$\begin{array}{ll}807000 & 559872 \\ 830601 & 575120\end{array}$

$\begin{array}{rr}0.9 & 1950 \\ 1.8 & 4099 \\ 2.9 & 6449 \\ 4.0 & 8999 \\ 5.2 & 11748 \\ 6.5 & 14698 \\ 7.9 & 17847 \\ 9.4 & 21197 \\ 10.9 & 24747 \\ 12.6 & 28498 \\ 14.3 & 32392 \\ 16.2 & 36595\end{array}$

347
1028
1856
2798
3810
4893
6017
7198
8452
9772

$18.2 \quad 41127 \quad 94055$

$\begin{array}{lll}20.3 & 46002 & 15660\end{array}$

$\begin{array}{lll}22.6 & 51229 & 17382 \\ 25.1 & 56829 & 19201\end{array}$

$27.7 \quad 62834 \quad 21132$

$\begin{array}{lll}30.6 & 69251 & 23181 \\ 33.6 & 76076 & 25345\end{array}$

$33.6 \quad 83303$

$40.1 \quad 90926$

$43.7 \quad 98914$

47.3107169

$51.0 \quad 115646$

$54.9 \quad 124311$

$62.7 \quad 102152$

$66.8 \quad 151346$

$70.9 \quad 160714$

75.2170301

$\begin{array}{ll}79.5 & 180103 \\ 83.9 & 190067\end{array}$

$\begin{array}{ll}83.9 & 190067 \\ 88.4 & 200245\end{array}$

$93.0 \quad 21054$

$97.6 \quad 221065$

107.0242170

$111.6 \quad 252624$

116.2263048

125.4283822

$130.0 \quad 294302$

$134.7 \quad 305042$
$139.6 \quad 316017$

$144.5 \quad 327152$

$149.5 \quad 338483$

$154.5 \quad 349670$

159.3360614

$169.0 \quad 382353$

$\begin{array}{rr}173.8 & 393303 \\ 178.7 & 404303\end{array}$

27621

30000

35005

37580

40161

45298
47856

47866
50430

53011

55600
58176

60761
63322

65891

6842

7079

73105
75341

77476

81674

83985

85145

90309

92333
94192

95967

97705

99428
101125

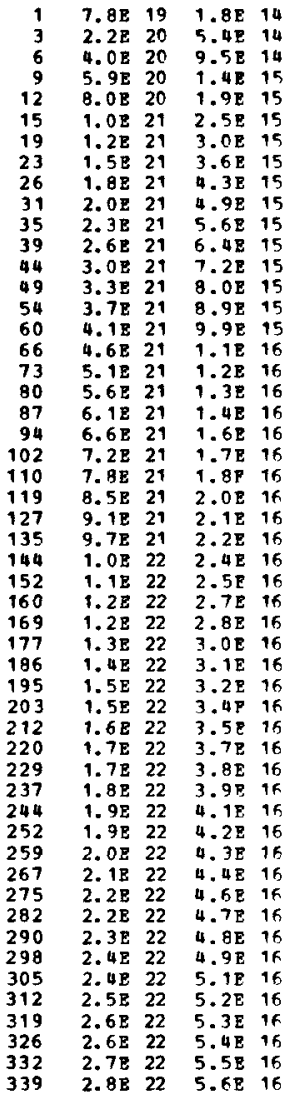

830601575120

$\begin{array}{ll}830601 & 575120 \\ 830601 & 575120\end{array}$

$\begin{array}{ll}830601 & 575120 \\ 830601 & 575120\end{array}$

830601575120

8330601575120

830601575120

$\begin{array}{ll}830601 & 575120 \\ 830601 & 575120\end{array}$

$\begin{array}{rrrrrrrr}178.7 & 404303 & 9417 & 89 & 1.7 \mathrm{E} & 22 & 5.2 \mathrm{E} & 15 \\ 178.7 & 404303 & 1507 & 46 & 1.1 \mathrm{E} & 22 & 3.7 \mathrm{E} & 14 \\ 178.7 & 404303 & 650 & 21 & 6.6 \mathrm{E} & 21 & 1.4 \mathrm{E} & 14 \\ 178.7 & 404303 & 291 & 9 & 4.4 \mathrm{E} & 21 & 5.7 \mathrm{E} & 13 \\ 178.7 & 404303 & 180 & 5 & 2.7 \mathrm{E} & 21 & 3.5 \mathrm{E} & 13 \\ 178.7 & 404303 & 75 & 2 & 1.0 \mathrm{E} & 21 & 2.0 \mathrm{E} & 13 \\ 178.7 & 404303 & 21 & 0 & 1.5 \mathrm{E} & 20 & 2.0 \mathrm{E} & 13 \\ 178.7 & 404303 & 13 & 0 & 3.3 \mathrm{E} & 19 & 2.2 \mathrm{E} & 13 \\ 178.7 & 404303 & 8 & 0 & 2.5 \mathrm{E} & 19 & 1.0 \mathrm{E} & 13\end{array}$


Table 3.26. Grans of light elenents accunlated at surf (shipped 5 years after discharge)

\begin{tabular}{|c|c|c|c|c|c|c|c|c|c|c|c|c|c|c|c|c|c|c|c|c|}
\hline \multirow{3}{*}{$\begin{array}{r}\text { H } \\
\text { HE } \\
\text { LI }\end{array}$} & \multicolumn{2}{|c|}{1990} & \multicolumn{2}{|c|}{1995} & \multicolumn{2}{|c|}{2000} & \multicolumn{2}{|c|}{2005} & \multicolumn{2}{|c|}{2010} & \multicolumn{2}{|c|}{2015} & \multicolumn{2}{|c|}{2020} & \multicolumn{2}{|c|}{2025} & \multicolumn{2}{|c|}{2030} & \multicolumn{2}{|l|}{2035} \\
\hline & $\begin{array}{l}6.07 \mathrm{E} \\
2.82 \mathrm{~B}\end{array}$ & $\begin{array}{l}04 \\
04\end{array}$ & & $\begin{array}{l}05 \\
04\end{array}$ & $\begin{array}{l}2.44 \mathrm{E} \\
1.18 \mathrm{E}\end{array}$ & $\begin{array}{l}05 \\
05\end{array}$ & $\begin{array}{l}3.978 \\
1.918\end{array}$ & $\begin{array}{l}05 \\
05\end{array}$ & $\begin{array}{l}5.92 \mathrm{E} \\
2.85 \mathrm{E}\end{array}$ & $\begin{array}{l}05 \\
05\end{array}$ & $\begin{array}{l}8.12 \mathrm{E} \\
3.89 \mathrm{E}\end{array}$ & $\begin{array}{l}05 \\
05\end{array}$ & $\begin{array}{l}1.05 \mathrm{E} \\
5.04 \mathrm{E}\end{array}$ & $\begin{array}{l}06 \\
05\end{array}$ & $\begin{array}{l}1.30 E \\
6.208\end{array}$ & $\begin{array}{l}06 \\
05\end{array}$ & $\begin{array}{l}9.558 \\
7.408\end{array}$ & $\begin{array}{l}06 \\
05\end{array}$ & $\begin{array}{l}1.81 \mathrm{E} \\
8.60 \mathrm{E}\end{array}$ & $\begin{array}{l}06 \\
05\end{array}$ \\
\hline & & 04 & & 04 & & O4 & $9.44 \mathrm{E}$ & 04 & 1.418 & 05 & $92 \mathrm{~B}$ & 05 & $2.49 \mathrm{E}$ & 05 & 3.068 & 05 & $3.66 \mathrm{E}$ & 05 & $4.25 E$ & 05 \\
\hline BE & $1.13 \mathrm{E}$ & 01 & $.60 \mathrm{E}$ & 01 & $4.68 \mathrm{~B}$ & 01 & $7.61 \mathrm{~B}$ & 01 & $1.13 \mathrm{E}$ & 02 & $.55 \mathrm{E}$ & 02 & $2.00 \mathrm{E}$ & 02 & $2.46 \mathrm{E}$ & 02 & $2.94 \mathrm{E}$ & 02 & $3.42 \mathrm{E}$ & 02 \\
\hline E & & 04 & & 04 & & ou & & 04 & & 05 & & 05 & & & & & $3.36 \mathrm{E}$ & 05 & $1.91 \mathrm{E}$ & 5 \\
\hline C & $2.56 \mathrm{E}$ & 06 & $.70 \mathrm{E}$ & 06 & 012 & 07 & $62 \mathrm{E}$ & 07 & $40 \mathrm{E}$ & 07 & $26 \mathrm{E}$ & 07 & $4.21 \mathrm{E}$ & 07 & $16 \bar{E}$ & 07 & $6.16 \mathrm{E}$ & 07 & .178 & 07 \\
\hline 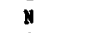 & & 06 & $4.10 \mathrm{E}$ & 06 & & 06 & 1.158 & 07 & & 07 & & 07 & $2.94 \mathrm{E}$ & 07 & $3.61 \mathrm{E}$ & 07 & $4.30 \mathrm{E}$ & 07 & & 07 \\
\hline 0 & & 09 & 4.498 & 09 & $7.88 \mathrm{~B}$ & 09 & $1.26 \mathrm{E}$ & 10 & $1.85 \mathrm{~B}$ & 10 & $.50 \mathrm{E}$ & 10 & $3.22 \mathrm{E}$ & 10 & $3.94 \mathrm{E}$ & 10 & $4.70 \mathrm{E}$ & 10 & $5.47 \mathrm{~B}$ & 10 \\
\hline $\mathbf{F}$ & & 05 & $3.14 \mathrm{E}$ & 05 & & 05 & $9.18 \mathrm{E}$ & 05 & & 06 & $1.87 \mathrm{E}$ & 06 & $2.42 \mathrm{E}$ & 06 & $2.98 \mathrm{E}$ & 06 & $3.56 \mathrm{E}$ & 06 & $4.14 \mathrm{E}$ & 06 \\
\hline ME & $4.78 \mathrm{~B}$ & 00 & $1.10 \mathrm{E}$ & 01 & $1.98 \mathrm{E}$ & 01 & $3.21 \mathrm{~B}$ & 01 & $4.78 \bar{E}$ & 01 & $6.54 \mathrm{E}$ & 01 & $8.47 \mathrm{E}$ & 01 & $1.04 E$ & 02 & $1.24 \mathrm{E}$ & 02 & $1.45 \mathrm{E}$ & 02 \\
\hline หล & & 05 & 4.398 & 05 & & 05 & $1.28 \mathrm{~B}$ & 06 & & 06 & & 06 & $3.39 \mathrm{E}$ & 06 & 4. $17 \mathrm{E}$ & 06 & & & 5.798 & 06 \\
\hline HG & $2.60 \mathrm{E}$ & 04 & $5.97 \mathrm{E}$ & 04 & $1.07 \mathrm{E}$ & 05 & 1.748 & 05 & 608 & 05 & $.55 E$ & 05 & $4.60 \mathrm{E}$ & 0 & $5.66 \mathrm{z}$ & & $76 \mathrm{~B}$ & 0 & $7.86 \mathrm{E}$ & 05 \\
\hline aI & & 06 & $2.42 \mathrm{~B}$ & 06 & & 06 & & 06 & & & & 07 & & 07 & $2.28 \mathrm{E}$ & & & & & 07 \\
\hline SI & $7.24 \mathrm{E}$ & 06 & $1.59 \mathrm{E}$ & 07 & $2.80 \mathrm{E}$ & 07 & $4.47 \mathrm{E}$ & 07 & 56B & 07 & $8.88 \mathrm{E}$ & 07 & $1.14 \mathrm{E}$ & 08 & $1.40 \mathrm{~B}$ & 0 & $1.67 \mathrm{E}$ & 08 & $1.94 \mathrm{E}$ & 08 \\
\hline$F$ & 3.368 & 06 & $7.61 E$ & 06 & $1.34 \mathrm{E}$ & 07 & $2.15 B$ & 07 & 3.1 & 07 & $4.24 \mathrm{E}$ & 07 & $5.44 \mathrm{E}$ & 07 & $6.66 \mathrm{E}$ & 07 & $7.93 \mathrm{E}$ & 07 & $9.18 \mathrm{E}$ & 07 \\
\hline c & 3.43E & 05 & $7.78 \mathrm{E}$ & 05 & & 06 & & 06 & & 06 & $64 \mathrm{~B}$ & 06 & $6.01 \mathrm{~B}$ & 0 & & & & 6 & $3 E$ & 07 \\
\hline CI & $6.79 \mathrm{E}$ & 04 & $1.56 \mathrm{E}$ & 05 & $2.80 \mathrm{E}$ & C5 & $4.56 \mathrm{E}$ & 05 & $8 z$ & 05 & 28E & 05 & $1.20 \mathrm{E}$ & 06 & $1.48 \mathrm{E}$ & 06 & $7 E$ & 06 & $05 \mathrm{E}$ & 06 \\
\hline $\begin{array}{l}\mathrm{A} R \\
\mathrm{~K}\end{array}$ & 1.72 & $\begin{array}{l}01 \\
00\end{array}$ & 3.5 & $\begin{array}{l}01 \\
00\end{array}$ & 7.108 & 01 & $1.15 \mathrm{E}$ & $\begin{array}{l}0 \\
0\end{array}$ & $\begin{array}{l}2 \mathrm{E} \\
1 \mathrm{E}\end{array}$ & $\begin{array}{l}02 \\
01\end{array}$ & & C & & 0 & $3.76 \mathrm{E}$ & & $49 \mathrm{~B}$ & & $5.23 \mathrm{E}$ & 02 \\
\hline$C_{A}^{n}$ & 568 & 04 & $5.87 E$ & 04 & & 0 & & & & 05 & & & & & & 0 & & & & 01 \\
\hline SC & & 00 & & 00 & & 00 & & 0 & & 01 & $\begin{array}{l}3.308 \\
1.76 E\end{array}$ & $\begin{array}{l}05 \\
01\end{array}$ & $\begin{array}{l}4.328 \\
2.27 \mathrm{E}\end{array}$ & $\begin{array}{l}03 \\
01\end{array}$ & & 01 & & $\begin{array}{l}05 \\
01\end{array}$ & $\begin{array}{l}7.738 \\
3.88 \mathrm{E}\end{array}$ & $\begin{array}{l}05 \\
01\end{array}$ \\
\hline$T I$ & $1.06 \mathrm{E}$ & 06 & $2.48 \mathrm{E}$ & 06 & $4.4 \mathrm{BB}$ & 06 & $7.27 \mathrm{~B}$ & 06 & 1. & 07 & & 07 & $1.90 \mathrm{E}$ & 07 & & 07 & 98 & 07 & $3.23 \mathrm{E}$ & 07 \\
\hline v & & 05 & & 05 & & 05 & $1.32 \mathrm{E}$ & 06 & $5 \mathrm{E}$ & 06 & & 06 & & 0 & $7 E$ & 06 & $5.10 \mathrm{E}$ & 06 & $5.93 \mathrm{E}$ & 06 \\
\hline$C R$ & $1.65 \mathrm{E}$ & 08 & 3.6 & 08 & & 08 & $1.03 \mathrm{E}$ & 09 & & 09 & & 0 & & 09 & & 09 & $82 \mathrm{E}$ & 09 & $4.44 E$ & 09 \\
\hline MN & 98 & 07 & $.05 \mathrm{E}$ & 07 & $5.36 \mathrm{E}$ & 07 & $8.58 \mathrm{E}$ & 07 & & 08 & & $O B$ & 2. & 08 & & 08 & $0 \mathrm{E}$ & 08 & $3.72 \mathrm{E}$ & 08 \\
\hline FE & & 08 & IIE & 09 & & 0 & 3 & 09 & & 09 & & & 7.9 & 09 & & & & & & 10 \\
\hline co & & 06 & .418 & 06 & $4.34 \mathrm{E}$ & & & 0 & & & & & & & & & & & & 07 \\
\hline HI & & 08 & $44 E$ & 08 & $6.06 \mathrm{E}$ & 08 & $69 \mathrm{E}$ & 08 & $1.42 \mathrm{E}$ & 09 & 918 & 09 & $2.46 \mathrm{E}$ & 09 & $3.01 \mathrm{E}$ & 09 & $58 \mathrm{E}$ & 09 & $16 \mathrm{E}$ & 09 \\
\hline $\mathrm{CD}$ & & 05 & $5.38 \mathrm{E}$ & 05 & 9.718 & 05 & $1.58 \mathrm{E}$ & 0 & $2.36 \mathrm{E}$ & 06 & & 0 & $22 \mathrm{~B}$ & 0 & 5.2 & 0 & $7 \mathrm{E}$ & 06 & $7.32 \mathrm{E}$ & 06 \\
\hline $2 x$ & & 05 & 1. $18 \mathrm{E}$ & 06 & $2.13 \mathrm{E}$ & 06 & $46 \mathrm{E}$ & 0 & & 06 & & 06 & $9.12 \mathrm{E}$ & 06 & $1.12 \mathrm{E}$ & 07 & $1.34 \mathrm{Z}$ & 07 & $1.56 \mathrm{E}$ & 07 \\
\hline GA & 3. & 02 & $9.18 \mathrm{E}$ & 02 & $1.65 \mathrm{E}$ & 03 & $2.68 E$ & 03 & & 03 & & 0 & $7.07 \mathrm{E}$ & 03 & & 03 & $1.04 \mathrm{E}$ & 0 & $1.21 E$ & 04 \\
\hline GP & & 00 & $.76 \mathrm{E}$ & 00 & $04 \mathrm{~B}$ & 01 & & 0 & & 01 & & 0 & $4.43 \mathrm{E}$ & 0 & & 0 & $1 E$ & 01 & $7.57 \mathrm{E}$ & 01 \\
\hline$S R$ & & 03 & 5E & 03 & & 03 & & 0 & & 04 & & & & & & & & & & 04 \\
\hline$Y$ & & -05 & $1 \mathrm{E}$ & -05 & & .05 & & -04 & & -04 & & -04 & 2. & -04 & 3. & -04 & $5 E$ & -04 & $09 E-$ & -04 \\
\hline $\mathrm{ZR}$ & & 09 & & 10 & & 10 & & 10 & & 10 & & 10 & & 10 & & 11 & & 1 & & 11 \\
\hline ME & & 06 & 1.748 & 07 & $3.07 \mathrm{~B}$ & 07 & $4.92 \mathrm{E}$ & 07 & & 07 & 2B & 07 & $1.25 \mathrm{~B}$ & $O B$ & $1.53 \mathrm{E}$ & 08 & $1.82 \mathrm{~B}$ & 08 & $2.11 \mathrm{E}$ & 08 \\
\hline nc & & 06 & $1.17 \mathrm{E}$ & 07 & $2.08 \mathrm{E}$ & 07 & $34 E$ & 07 & $4.91 \mathrm{E}$ & 07 & & 07 & & 07 & $1.05 E$ & 08 & $5 \mathrm{E}$ & 08 & $1.45 \mathrm{E}$ & 08 \\
\hline TC & & 03 & $7.39 E$ & 03 & $1.34 \mathrm{~B}$ & 04 & & 04 & & 04 & & 04 & & 04 & & 04 & & & $9.62 \mathrm{E}$ & 04 \\
\hline RO & 1.318 & 03 & $3.09 \mathrm{E}$ & 03 & & 03 & & 03 & & 04 & & 04 & & 04 & $2.91 \mathrm{E}$ & O4 & $7 \mathrm{E}$ & 04 & $4.02 \mathrm{E}$ & 04 \\
\hline$C D$ & $1.33 \mathrm{E}$ & 01 & & 01 & & 01 & & 01 & & 02 & & 02 & & 02 & & 02 & & 02 & & 02 \\
\hline IN & $1.03 \mathrm{E}$ & 04 & $2.32 \mathrm{E}$ & 04 & $4.16 \mathrm{~B}$ & 04 & $6.76 \mathrm{E}$ & or & & 05 & & 05 & & 0 & $2.21 \mathrm{~B}$ & 05 & & 0 & $3.08 \mathrm{E}$ & 05 \\
\hline SN & $8.51 \mathrm{E}$ & 07 & $1.84 E$ & 08 & $3.22 \mathrm{E}$ & 08 & 5.168 & 08 & & 08 & & 09 & & 09 & $1.63 \mathrm{E}$ & 09 & & 09 & 2.278 & 09 \\
\hline $\mathrm{SE}$ & 718 & 04 & $8.34 E$ & 04 & & 05 & & 0 : & & 05 & & 05 & & 05 & & 05 & & 05 & $1.05 \mathrm{E}$ & 06 \\
\hline TR & & 04 & & 04 & & 05 & & 0 & & 05 & & & & 0 & & 05 & $1 \mathrm{~B}$ & 0 & & 06 \\
\hline TA & & 03 & & 04 & & & & & & & & & & & & & $2 E$ & & & \\
\hline 10 & $10 E$ & 05 & $2.47 \mathrm{E}$ & 05 & $4.43 \mathrm{~B}$ & 05 & $7.20 \mathrm{E}$ & 0 & & 06 & & 06 & & 06 & $2.35 \mathrm{E}$ & 06 & $11 \mathrm{E}$ & 06 & $28 \mathrm{E}$ & 06 \\
\hline TOT & & 09 & $1.80 \mathrm{E}$ & 10 & $3.15 \mathrm{E}$ & 10 & & 10 & $40 \mathrm{E}$ & 10 & & 71 & $9 \mathrm{~B}$ & 11 & $1.59 \mathrm{~B}$ & 11 & $1.89 \mathrm{E}$ & 11 & $2.20 \mathrm{E}$ & 11 \\
\hline
\end{tabular}


Table 3.27. Grans of fission-product eleents accueulated at soRrF (shipped 5 years after discharge)

\begin{tabular}{|c|c|c|c|c|c|c|c|c|c|c|c|c|}
\hline & 1990 & 1995 & & 2000 & 2005 & 2010 & 2015 & & 2020 & 2025 & 2030 & 2035 \\
\hline & & & 02 & & & & & & & $5.52 E$ & & $6.48 \mathrm{E}$ \\
\hline & & $1.81 E$ & 04 & 26804 & $30 E$ & $7.88 \mathrm{~B}$ & & & & & B & \\
\hline & IE 0 & $5.49 \mathrm{E}$ & 03 & 88803 & .618 & $2.395 \quad 04$ & 271 & & $23 \mathrm{E} 0$ & 5.2 & 18 & IE O4 \\
\hline & 5 E 05 & $1.56 \mathrm{E}$ & 06 & & 4. & $6.77 \mathrm{E} \quad 06$ & $9.26 \mathrm{E}$ & & $.20 \mathrm{E} 07$ & $1.47 \mathrm{E} 07$ & $\begin{array}{lll}1.76 \mathrm{~B} & 07\end{array}$ & \\
\hline & & $5.99 \mathrm{E}$ & 05 & .08506 & & 2.61806 & $3.56 \mathrm{E}$ & & $61=06$ & $5.67 \mathrm{E} 06$ & $6.77 \mathrm{E} \quad 06$ & \\
\hline & $4.28 E$ O6 & $9.86 \mathrm{E}$ & 06 & $.778 \quad 07$ & & 4.27807 & & & & $9.22 E 07$ & 1.108 og & $1.28 \mathrm{E}$ \\
\hline & $4.22 \mathrm{E} 06$ & 9.742 & 06 & & & $4.26 \mathrm{E} 0$ & & & & $9.34 \mathrm{E}$ & $1.12 \mathrm{E} \quad 08$ & $1.30 E$ \\
\hline & & 2.108 & 07 & & 9780 & 8.7420 & & & $1.49 \mathrm{E}$ & $1.79 \mathrm{E} 08$ & & $2.38 \mathrm{~B}$ \\
\hline & $5.47 \mathrm{E} 06$ & $1.26 \mathrm{E}$ & 07 & $2.27 \mathrm{E}$ & $3.69 \mathrm{E}$ & $5.48 \mathrm{E} 0$ & 7.5 & & 9.7080 & $1.19 \mathrm{E} \quad 08$ & $1.42 \mathrm{~B} 0$ & $1.66 \mathrm{~B}$ \\
\hline $\mathbf{z}$ & 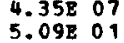 & $\begin{array}{l}1.00 E \\
1.228\end{array}$ & $\begin{array}{l}08 \\
02\end{array}$ & $\begin{array}{l}\text { E } \\
\text { E }\end{array}$ & $\begin{array}{l}2.95 \mathrm{E} \\
4.21 \mathrm{E}\end{array}$ & 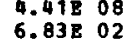 & $\begin{array}{l}6.05 \mathrm{E} \\
1.03 \mathrm{~B}\end{array}$ & $\begin{array}{l}08 \\
03\end{array}$ & $\begin{array}{l}7.86 \mathrm{E} 0 \\
1.45 \mathrm{E}\end{array}$ & $\begin{array}{ll}9.708 & 08 \\
1.978 & 03\end{array}$ & $\begin{array}{rl}1.168 & 09 \\
2.578 & 03\end{array}$ & $\begin{array}{ll}1.36 \mathrm{~B} & 09 \\
3.25 \mathrm{~B} & 03\end{array}$ \\
\hline & 3.9 & & 07 & & & $4.00 \mathrm{E} 08$ & & 08 & $7.08 \mathrm{E}$ & $8.71 E$ O8 & $1.04 \mathrm{E}$ & $1.21 E$ O9 \\
\hline & 06 & & 07 & & & & & 08 & $1.66 \mathrm{E}$ & & & \\
\hline & 2. & & 07 & & & & & & & & & 7.90808 \\
\hline & 5.7 & $1.32 \mathrm{E}$ & 07 & 807 & & & & & & & & \\
\hline & & BE & 07 & & & 1. & & & & & & \\
\hline & & 4E & 06 & 06 & & 9. & & & & & & 2 \\
\hline & & & & & & & & & & & & \\
\hline & E O4 & 7.0 & 04 & 05 & & & & & & 6. & & \\
\hline & & & 06 & & & & & & & & & \\
\hline & & & & 9.5 & & & & & & & & \\
\hline & & & & & & & & & & & & \\
\hline & 2.8 & 6.5 & 0 & 1.1 & & & & & 5. & & & \\
\hline & 6.3 & 1.4 & 08 & 2.5 & & 6. & & & & & & \\
\hline & 2.5 & 6.6 & 07 & 1.1 & 1.5 & 2. & 3.7 & of & 4.8 & & & \\
\hline & 2. & & & 8.7 & & 2. & & 0 & & & & \\
\hline & & & & & & & & 0 & & & & \\
\hline & 2 & & & & & & & & & & & \\
\hline & & & & & & & & & & & & \\
\hline & & & & & & & & & & & & \\
\hline & & & & & & & & & & & & \\
\hline & & & & & & & 1.1 & & & & & \\
\hline & 1.61806 & $3.70 \mathrm{E}$ & 06 & 6.6 & 1.0 & 1.5 & $2.13 \mathrm{E}$ & & 2.7 & 3.3480 & $5=0$ & \\
\hline & $1.29 \mathrm{E} \quad 06$ & & 06 & 5.4 & 8.9 & 1.3 & $1.85 \mathrm{E}$ & & 2.4 & & & 4.2 \\
\hline & & & & & $E 0$ & 3.0 & 4.1 & & 5.4 & $6.66 \mathrm{E} 0$ & 7.9 & $9.24 \mathrm{E}$ \\
\hline & & & & & & & $2.25 \mathrm{E}$ & & $2.91 \bar{E}$ & $3.57 \mathrm{E}$ & $4.27 \bar{B}$ & $4.96 \mathrm{E}$ \\
\hline H & & $3.69 \mathrm{E}$ & 0 & & & & & & & $3.49 \mathrm{E}$ & $4.17 \mathrm{E}$ & $4.84 \mathrm{E}$ \\
\hline & $5.45 E$ & & 0 & & & & & & & & & \\
\hline & 4.09808 & $9.42 \mathrm{E}$ & & $1.70 E 09$ & $2.75 \mathrm{E} 0$ & $4.70 \mathrm{E} \quad 09$ & $5.60 \mathrm{E}$ & & $7.25 E 09$ & $8.92 E 09$ & $1.06 \mathrm{E} 10$ & $1.24 \mathrm{E} 10$ \\
\hline
\end{tabular}

Table 3.28. Grass of heavy elements accumulated at SURPP (shipped 5 years after discharge)

$\begin{array}{llllllllll}1990 & 1995 & 2000 & 2005 & 2010 & 2015 & 2020 & 2025 & 2030 & 2035\end{array}$

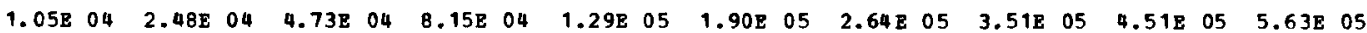

$\begin{array}{lllllllllll}2.23 E-07 & 5.12 E-07 & 9.39 E-07 & 1.54 E-06 & 2.32 E-06 & 3.19 E-06 & 4.14 E-06 & 5.08 E-06 & 6.02 E-06 & 6.938-06\end{array}$

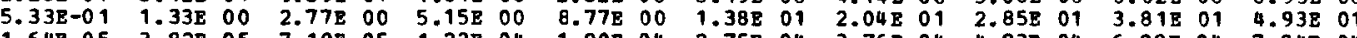

$\begin{array}{llllllllll}1.64 \mathrm{E}-05 & 3.82 \mathrm{E}-05 & 7.19 \mathrm{E}-05 & 1.22 \mathrm{E}-04 & 1.90 \mathrm{E}-04 & 2.75 \mathrm{E}-04 & 3.76 \mathrm{E}-04 & 4.93 \mathrm{E}-04 & 6.28 \mathrm{E}-04 & 7.84 \mathrm{E}-04\end{array}$

$\begin{array}{llllllllll}1.36 \mathrm{E}-07 & 3.96 \mathrm{E}-07 & 9.56 \mathrm{E}-07 & 2.06 \mathrm{E}-06 & 4.04 \mathrm{E}-06 & 7.37 \mathrm{E}-06 & 1.26 \mathrm{E}-05 & 2.04 \mathrm{E}-05 & 3.15 \mathrm{~B}-05 & 4.68 \mathrm{E}-05\end{array}$

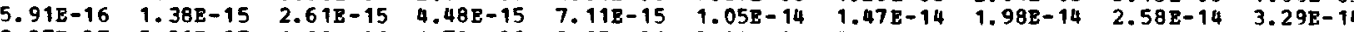

$\begin{array}{llllllllll}2.27 E-07 & 5.31 \mathrm{E}-07 & 1.00 \mathrm{E}-06 & 1.70 \mathrm{E}-06 & 2.65 \mathrm{E}-06 & 3.82 \mathrm{E}-06 & 5.22 \mathrm{E}-06 & 6.83 \mathrm{E}-06 & \mathbf{8 . 6 9 E}-06 & 1.08 \mathrm{E}-05\end{array}$

$\begin{array}{llllllllll}2.93 \mathrm{E}-11 & 7.06 \mathrm{E}-11 & 1.39 \mathrm{E}-10 & 2.46 \mathrm{E}-10 & 3.99 \mathrm{E}-10 & 6.01 \mathrm{E}-10 & 8.55 \mathrm{E}-10 & 1.16 \mathrm{E}-09 & 1.51 \mathrm{E}-09 & 1.92 \mathrm{E}-09 \\ 5.84 \mathrm{E}-03 & 1.49 \mathrm{E}-02 & 3.14 \mathrm{E}-02 & 5.98 \mathrm{E}-02 & 1.05 \mathrm{E}-01 & 1.72 \mathrm{E}-01 & 2.66 \mathrm{E}-01 & 3.92 \mathrm{E}-01 & 5.58 \mathrm{E}-01 & 7.68 \mathrm{E}-01\end{array}$

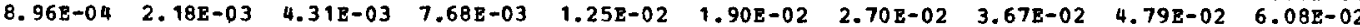

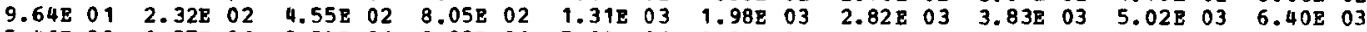

$\begin{array}{llllllllllllllllllll}5.41 E & 00 & 1.27 \mathrm{E} & 01 & 2.34 \mathrm{E} & 01 & 3.88 \mathrm{E} & 01 & 5.92 \mathrm{E} & 01 & 8.34 \mathrm{E} & 01 & 1.11 \mathrm{E} & 02 & 1.41 \mathrm{E} & 02 & 1.75 \mathrm{E} & 02 & 2.10 \mathrm{E} & 02\end{array}$

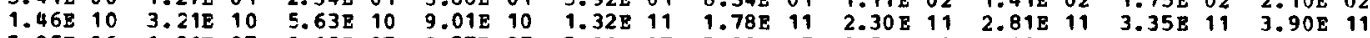

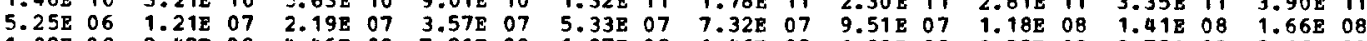

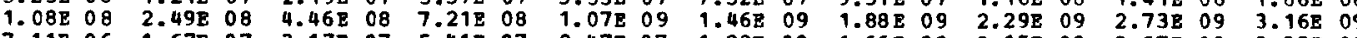

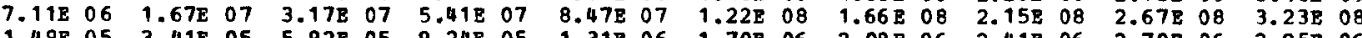

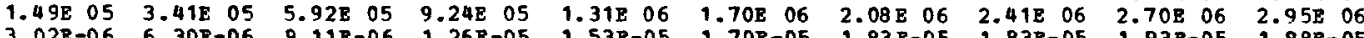

$\begin{array}{llllllllll}3.02 \mathrm{E}-06 & 6.30 \mathrm{E}-06 & 9.11 \mathrm{E}-06 & 1.26 \mathrm{E}-05 & 1.53 \mathrm{E}-05 & 1.70 \mathrm{E}-05 & 1.83 \mathrm{E}-05 & 1.83 \mathrm{E}-05 & 1.93 \mathrm{E}-05 & 1.88 \mathrm{E}-05\end{array}$

$\begin{array}{llllllllll}1.25 \mathrm{E}-03 & 2.91 \mathrm{E}-03 & 5.22 \mathrm{E}-03 & 8.42 \mathrm{E}-03 & 1.24 \mathrm{E}-02 & 1.68 \mathrm{E}-02 & 2.15 \mathrm{E}-02 & 2.61 \mathrm{E}-02 & 3.09 \mathrm{E}-02 & 3.55 \mathrm{E}-02\end{array}$

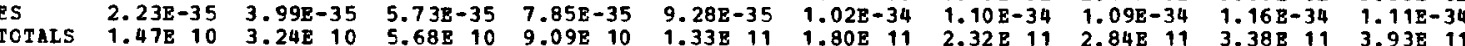


Table 3.29. Curies of light-elenent isotopes accululated at suRp (shipped 5 years after discharge)

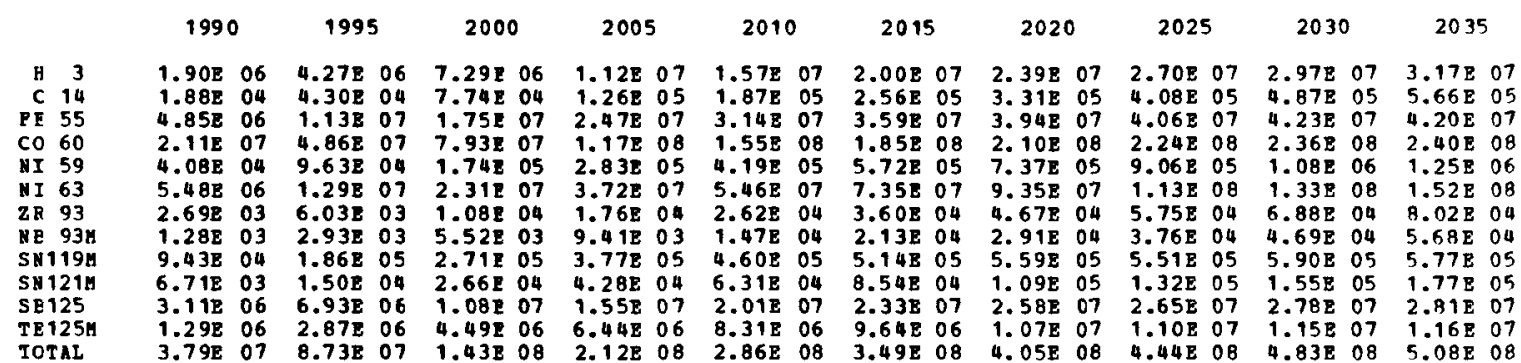

Table 3.30. Curies of fission-product isotopes accunulated at sorfF (shipped 5 years after discharge)

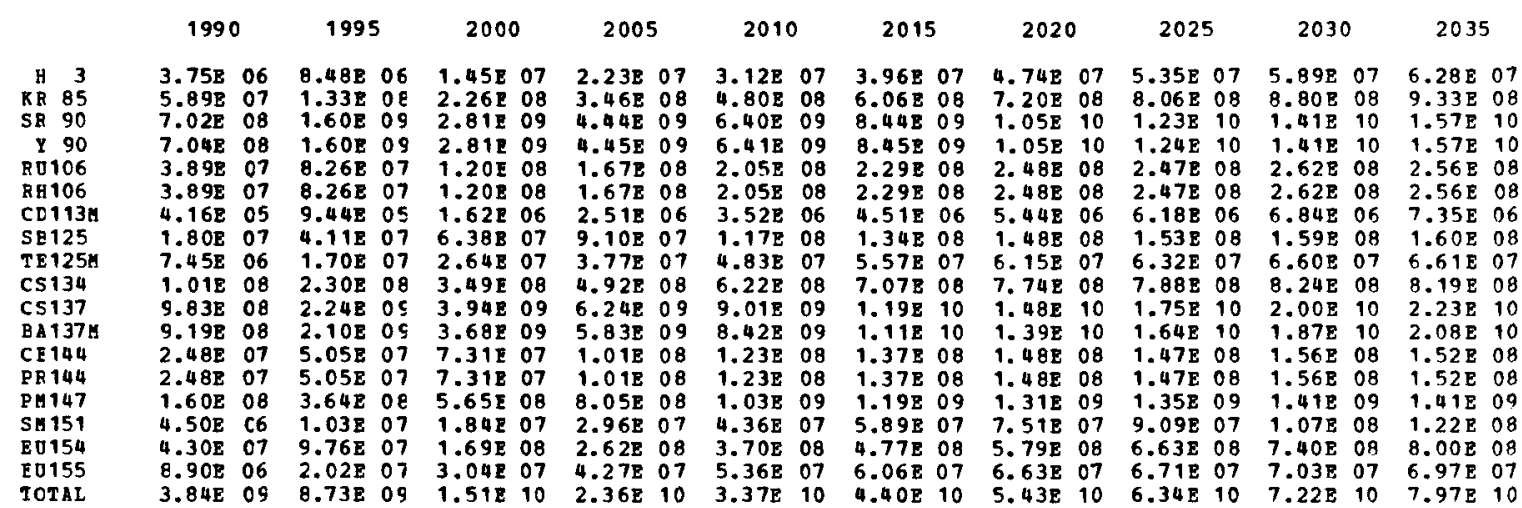

Table 3.31. Curies of heavy-elewent isotopes accumulated at SURPP (shipped 5 years after discharge)

$\begin{array}{llllllllll}1990 & 1995 & 2000 & 2005 & 2010 & 2015 & 2020 & 2025 & 2030 & 2035\end{array}$

\begin{tabular}{|c|c|c|c|c|c|c|c|c|c|c|c|c|c|c|c|c|c|c|c|}
\hline $\begin{array}{r}0234 \\
\text { NP239 } \\
\text { PU238 } \\
\text { PO239 } \\
\text { P } 0240 \\
\text { PU241 } \\
\text { PO242 } \\
\text { A } 241 \\
\text { A } 2424 \\
\text { AH2 } 243 \\
\text { CH242 } \\
\text { CH243 } \\
\text { CH2 } 44 \\
\text { TOTAL }\end{array}$ & $\begin{array}{l}1.43 E \\
1.92 E \\
2.51 E \\
3.89 E \\
6.32 E \\
9.44 E \\
2.15 E \\
2.09 E \\
5.33 E \\
1.91 E \\
6.33 E \\
1.22 E \\
1.17 E \\
1.01 E\end{array}$ & $\begin{array}{l}04 \\
05 \\
07 \\
06 \\
06 \\
08 \\
04 \\
07 \\
04 \\
05 \\
04 \\
05 \\
07 \\
09\end{array}$ & $\begin{array}{l}3.30 \mathrm{E} \\
4.42 \mathrm{E} \\
5.77 \mathrm{E} \\
8.93 \mathrm{E} \\
1.45 \mathrm{E} \\
2.13 \mathrm{E} \\
4.95 \mathrm{E} \\
4.93 \mathrm{E} \\
1.22 \mathrm{E} \\
4.42 \mathrm{E} \\
1.37 \mathrm{E} \\
2.78 \mathrm{E} \\
2.67 \mathrm{E} \\
2.29 \mathrm{E}\end{array}$ & $\begin{array}{l}04 \\
05 \\
07 \\
06 \\
07 \\
09 \\
04 \\
07 \\
05 \\
05 \\
05 \\
05 \\
07 \\
09\end{array}$ & $\begin{array}{l}5.98 \mathrm{E} \\
7.96 \mathrm{E} \\
1.03 \mathrm{E} \\
1.61 \mathrm{E} \\
2.61 \mathrm{E} \\
3.67 \mathrm{E} \\
8.91 \mathrm{E} \\
9.43 \mathrm{E} \\
2.18 \mathrm{E} \\
7.95 \mathrm{E} \\
2.32 \mathrm{E} \\
4.89 \mathrm{~B} \\
4.63 \mathrm{E} \\
3.96 \mathrm{E}\end{array}$ & $\begin{array}{l}04 \\
05 \\
08 \\
07 \\
07 \\
09 \\
04 \\
07 \\
05 \\
05 \\
05 \\
05 \\
07 \\
09\end{array}$ & $\begin{array}{l}9.77 \mathrm{E} \\
1.29 \mathrm{E} \\
1.66 \mathrm{E} \\
2.61 \mathrm{E} \\
4.25 \mathrm{E} \\
5.69 \mathrm{E} \\
1.45 \mathrm{E} \\
1.62 \mathrm{E} \\
3.52 \mathrm{E} \\
1.29 \mathrm{E} \\
3.63 \mathrm{E} \\
7.76 \mathrm{E} \\
7.22 \mathrm{E} \\
6.16 \mathrm{E}\end{array}$ & $\begin{array}{l}04 \\
06 \\
08 \\
07 \\
07 \\
09 \\
05 \\
08 \\
05 \\
06 \\
05 \\
05 \\
07 \\
09\end{array}$ & $\begin{array}{l}1.46 \mathrm{E} \\
1.92 \mathrm{E} \\
2.44 \mathrm{E} \\
3.89 \mathrm{E} \\
6.32 \mathrm{E} \\
8.02 \mathrm{E} \\
2.15 \mathrm{E} \\
2.56 \mathrm{E} \\
5.21 \mathrm{E} \\
1.92 \mathrm{E} \\
5.16 \mathrm{E} \\
1.12 \mathrm{E} \\
1.03 \mathrm{E} \\
8.73 \mathrm{E}\end{array}$ & $\begin{array}{l}05 \\
06 \\
08 \\
07 \\
07 \\
09 \\
05 \\
08 \\
05 \\
06 \\
05 \\
06 \\
08 \\
09\end{array}$ & $\begin{array}{l}2.02 \mathrm{E} \\
2.63 \mathrm{E} \\
3.30 \mathrm{E} \\
5.32 \mathrm{E} \\
8.65 \mathrm{E} \\
1.03 \mathrm{E} \\
2.95 \mathrm{E} \\
3.72 \mathrm{E} \\
7.08 \mathrm{E} \\
2.63 \mathrm{E} \\
6.79 \mathrm{E} \\
1.49 \mathrm{E} \\
1.33 \mathrm{E} \\
1.13 \mathrm{E}\end{array}$ & $\begin{array}{l}05 \\
06 \\
08 \\
07 \\
07 \\
10 \\
05 \\
08 \\
05 \\
06 \\
05 \\
06 \\
08 \\
10\end{array}$ & $\begin{array}{l}2.63 E \\
3.40 E \\
4.20 E \\
6.88 E \\
1.12 E \\
1.24 E \\
3.81 E \\
5.09 E \\
9.10 E \\
3.40 E \\
8.53 E \\
1.86 E \\
1.62 E \\
1.37 E\end{array}$ & $\begin{array}{l}05 \\
06 \\
08 \\
07 \\
08 \\
10 \\
05 \\
08 \\
05 \\
06 \\
05 \\
06 \\
08 \\
10\end{array}$ & $\begin{array}{l}3.26 \mathrm{E} \\
4.18 \mathrm{E} \\
5.09 \mathrm{E} \\
8.46 \mathrm{E} \\
1.38 \mathrm{E} \\
1.42 \mathrm{~B} \\
4.69 \mathrm{E} \\
6.61 \mathrm{E} \\
1.11 \mathrm{~B} \\
4.17 \mathrm{E} \\
1.09 \mathrm{E} \\
2.19 \mathrm{E} \\
1.87 \mathrm{E} \\
1.58 \mathrm{E}\end{array}$ & $\begin{array}{l}05 \\
06 \\
08 \\
07 \\
08 \\
10 \\
05 \\
08 \\
06 \\
06 \\
06 \\
06 \\
08 \\
10\end{array}$ & $\begin{array}{l}3.93 \mathrm{E} \\
4.99 \mathrm{E} \\
5.99 \mathrm{E} \\
1.01 \mathrm{E} \\
1.64 \mathrm{E} \\
1.57 \mathrm{E} \\
5.60 \mathrm{E} \\
8.27 \mathrm{E} \\
1.31 \mathrm{E} \\
4.98 \mathrm{~B} \\
1.19 \mathrm{E} \\
2.52 \mathrm{E} \\
2.09 \mathrm{E} \\
1.77 \mathrm{E}\end{array}$ & $\begin{array}{l}05 \\
06 \\
08 \\
08 \\
08 \\
10 \\
05 \\
08 \\
06 \\
06 \\
06 \\
06 \\
08 \\
10\end{array}$ & $\begin{array}{l}4.61 \mathrm{E} \\
5.79 \mathrm{E} \\
6.84 \mathrm{E} \\
1.18 \mathrm{E} \\
1.91 \mathrm{E} \\
1.70 \mathrm{E} \\
6.51 \mathrm{E} \\
1.00 \mathrm{E} \\
1.51 \mathrm{E} \\
5.79 \mathrm{E} \\
1.35 \mathrm{E} \\
2.81 \mathrm{E} \\
2.28 \mathrm{E} \\
1.92 \mathrm{E}\end{array}$ \\
\hline
\end{tabular}


Table 3.32. Natts of light-elenent isotopes accunulated at SORPP (shipped 5 years after discharge)

\begin{tabular}{|c|c|c|c|c|c|c|c|c|c|c|}
\hline & 1990 & 1995 & 2000 & 2005 & 2010 & 2015 & 2020 & 2025 & 2030 & 2035 \\
\hline $\begin{array}{ll}\text { H } & 3 \\
\text { C } & 14 \\
\text { PE } & 55 \\
\text { CO } & 60 \\
\text { NI } & 63 \\
\text { ZR } & 93 \\
\text { NB } & 93 \text { 93 } \\
\text { SN 198 } \\
\text { SN1211 } \\
\text { SE125 } \\
\text { IE 125 } \\
\text { TOTAL }\end{array}$ & $\begin{array}{lll}6.74 E & 01 \\
5.56 \mathrm{E} & 00 \\
6.32 \mathrm{E} & 03 \\
3.29 \mathrm{E} & 05 \\
8.77 \mathrm{E} & 02 \\
3.18 \mathrm{E} & -01 \\
4.46 \mathrm{E}-01 \\
4.98 \mathrm{E} & 01 \\
7.04 \mathrm{O} & 00 \\
1.26 \mathrm{O} & 04 \\
1.11 \mathrm{E} & 03 \\
3.50 \mathrm{E} & 05\end{array}$ & 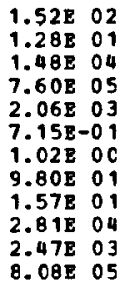 & 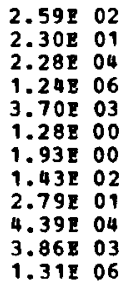 & $\begin{array}{ll}3.998 & 02 \\
3.73 E & 01 \\
3.228 & 04 \\
1.828 & 06 \\
1.928 & 03 \\
5.958 & 03 \\
2.088 & 00 \\
3.298 & 00 \\
1.998 & 02 \\
4.498 & 01 \\
6.30 E & 04 \\
5.538 & 03 \\
1.938 & 06\end{array}$ & $\begin{array}{lll}5.598 & 02 \\
5.55 \mathrm{~B} & 01 \\
4.10 \mathrm{~B} & 04 \\
2.42 \mathrm{~B} & 06 \\
8.73 \mathrm{~B} & 03 \\
3.11 \mathrm{E} & 00 \\
5.16 \mathrm{~B} & 00 \\
2.43 \mathrm{~B} & 02 \\
6.62 \mathrm{~B} & 01 \\
8.14 \mathrm{E} & 04 \\
7.15 \mathrm{~B} & 03 \\
2.56 \mathrm{~B} & 06\end{array}$ & $\begin{array}{lll}7.10 \mathrm{E} & 02 \\
7.59 \mathrm{E} & 01 \\
4.69 \mathrm{E} & 04 \\
2.89 \mathrm{E} & 06 \\
1.18 \mathrm{E} & 04 \\
4.27 \mathrm{~B} & 00 \\
7.46 \mathrm{~B} & 00 \\
2.71 \mathrm{~B} & 02 \\
8.96 \mathrm{~B} & 01 \\
9.43 \mathrm{E} & 04 \\
8.29 \mathrm{E} & 03 \\
3.06 \mathrm{~B} & 06\end{array}$ & $\begin{array}{lll}8.51 E & 02 \\
9.82 E & 01 \\
5.14 E & 04 \\
3.28 E & 06 \\
1.502 & 04 \\
5.54 E & 00 \\
1.02 E & 01 \\
2.95 E & 02 \\
1.15 E & 02 \\
1.05 E & 05 \\
9.208 & 03 \\
3.46 E & 06\end{array}$ & $\begin{array}{lll}9.61 E & 02 \\
1.21 E & 02 \\
5.29 E & 04 \\
3.50 E & 06 \\
1.81 E & 04 \\
6.82 E & 00 \\
1.31 E & 01 \\
2.91 E & 02 \\
1.38 E & 02 \\
1.08 E & 05 \\
9.44 E & 03 \\
3.69 E & 06\end{array}$ & $\begin{array}{lll}1.06 \mathrm{E} & 03 \\
1.44 \mathrm{E} & 02 \\
5.51 \mathrm{E} & 04 \\
3.69 \mathrm{E} & 06 \\
2.13 \mathrm{E} & 04 \\
8.16 \mathrm{E} & 00 \\
1.64 \mathrm{E} & 01 \\
3.11 \mathrm{E} & 02 \\
1.63 \mathrm{E} & 02 \\
1.13 \mathrm{E} & 05 \\
9.90 \mathrm{E} & 03 \\
3.89 \mathrm{E} & 06\end{array}$ & $\begin{array}{ll}1.13 \mathrm{E} & 03 \\
1.6 \mathrm{E} & 02 \\
5.47 \mathrm{E} & 04 \\
3.76 \mathrm{E} & 06 \\
2.43 \mathrm{E} & 04 \\
9.51 \mathrm{E} & 00 \\
1.99 \mathrm{E} & 01 \\
3.04 \mathrm{E} & 02 \\
1.86 \mathrm{E} & 02 \\
1.14 \mathrm{E} & 05 \\
1.00 \mathrm{E} & 04 \\
3.96 \mathrm{E} & 06\end{array}$ \\
\hline
\end{tabular}

Table 3.33. Watts of fission-product isotopes accusulated at SORFP (shipped 5 years after discharge)

$\begin{array}{llllllllll}1990 & 1995 & 2000 & 2005 & 2010 & 2015 & 2020 & 2025 & 2030 & 2035\end{array}$

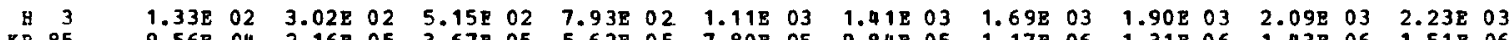

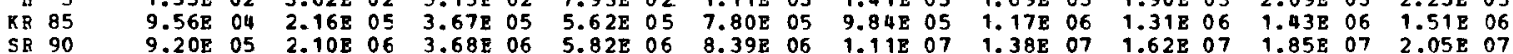

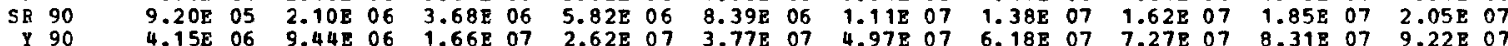

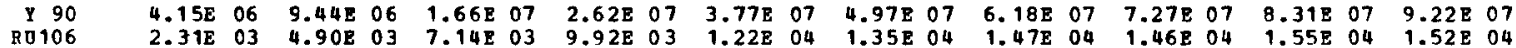

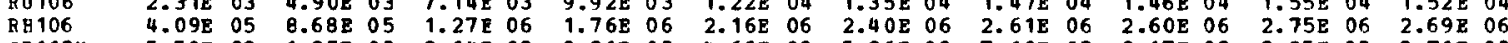

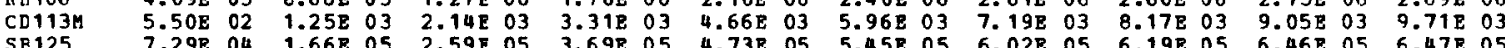

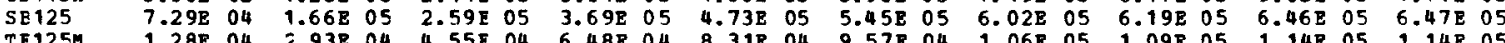

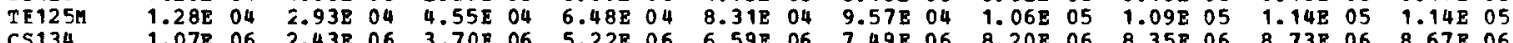

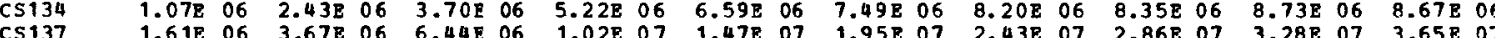

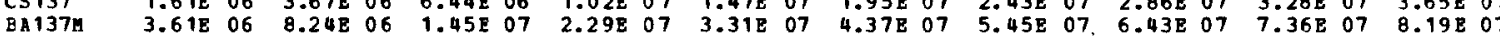

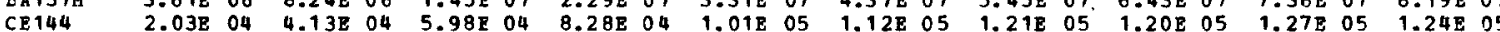

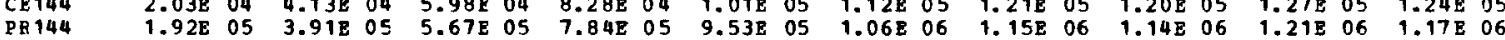

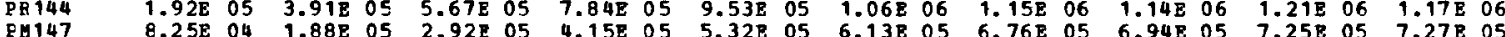

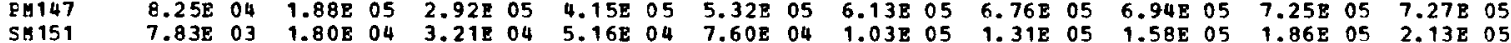

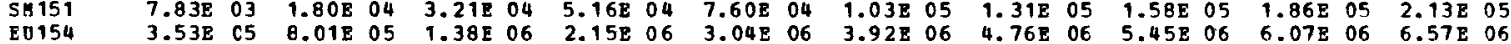

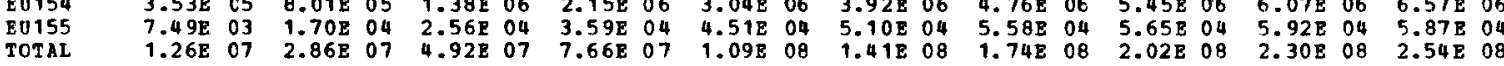

Table 3.34. Natts of heavy-elenent isotopes accunulated at sURF (shipped 5 years after discharge)

$\begin{array}{llllllllll}1990 & 1995 & 2000 & 2005 & 2010 & 2015 & 2020 & 2025 & 2030 & 2035\end{array}$

$\begin{array}{lllllllllllllllllllllll}4.12 \mathrm{E} & 02 & 9.51 \mathrm{E} & 02 & 1.72 \mathrm{E} & 03 & 2.81 \mathrm{E} & 03 & 4.21 \mathrm{E} & 03 & 5.80 \mathrm{E} & 03 & 7.57 \mathrm{E} & 03 & 9.39 \mathrm{E} & 03 & 1.13 \mathrm{E} & 04 & 1.33 \mathrm{E} & 04\end{array}$

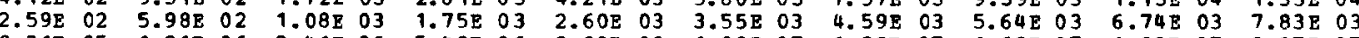

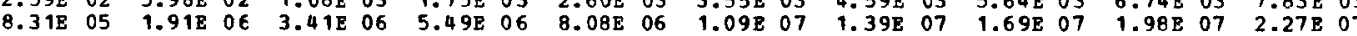

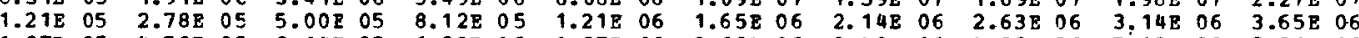

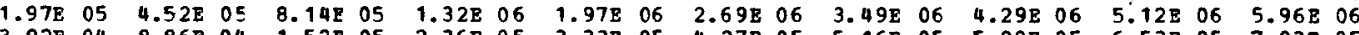
$\begin{array}{llllllllllllllllllll}3.92 E & 04 & 8.86 E & 04 & 1.52 E & 05 & 2.36 E & 05 & 3.33 E & 05 & 4.27 E & 05 & 5.16 E & 05 & 5.88 E & 05 & 6.53 E & 05 & 7.03 E & 05\end{array}$

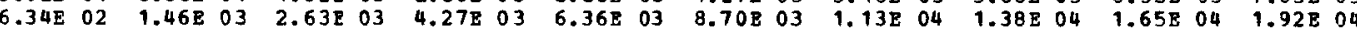
$\begin{array}{llllllllllllllllllll}6.99 \mathrm{E} & 05 & 1.65 \mathrm{E} & 06 & 3.15 \mathrm{E} & 06 & 5.41 \mathrm{E} & 06 & 8.54 \mathrm{E} & 06 & 1.24 \mathrm{E} & 07 & 1.70 \mathrm{E} & 07 & 2.21 \mathrm{E} & 07 & 2.76 \mathrm{E} & 07 & 3.35 \mathrm{E} & 07\end{array}$ $\begin{array}{llllllllllllllllllllll}1.52 \mathrm{E} & 01 & 3.47 \mathrm{E} & 01 & 6.20 \mathrm{E} & 01 & 1.00 \mathrm{E} & 02 & 1.48 \mathrm{E} & 02 & 2.02 \mathrm{E} & 02 & 2.59 \mathrm{E} & 02 & 3.15 \mathrm{E} & 02 & 3.74 \mathrm{E} & 02 & 4.30 \mathrm{E} & 02\end{array}$

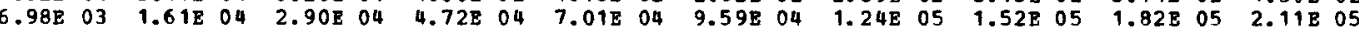
$\begin{array}{lllllllllllllllllllllllll}2.33 E & 03 & 5.04 \mathrm{E} & 03 & 8.55 \mathrm{E} & 03 & 1.34 \mathrm{E} & 04 & 1.90 \mathrm{~B} & 04 & 2.50 \mathrm{E} & 04 & 3.148 & 04 & 3.74 \mathrm{E} & 04 & 4.38 \mathrm{E} & 04 & 4.97 \mathrm{E} & 04\end{array}$

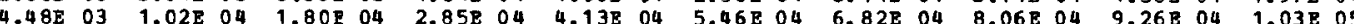

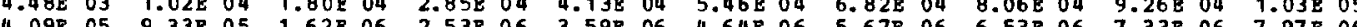

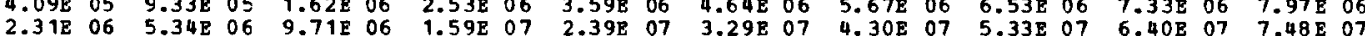


Table 3.35. Ingestion toxicity $\left(\mathrm{a}^{3}\right.$ vater) of light-elenent isotopes accuoulated at soRF (shipped 5 years after aischarge)

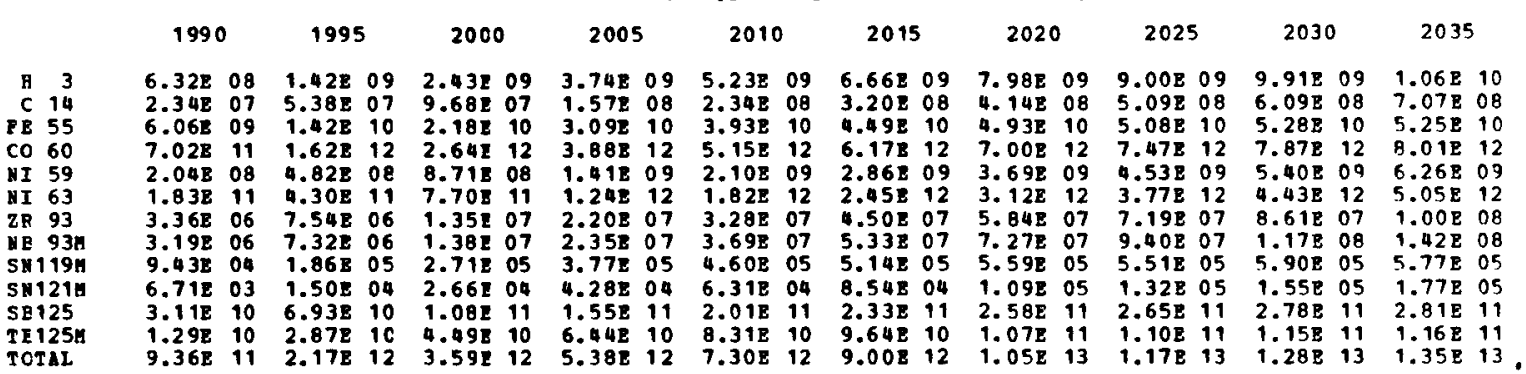

Table 3.36. Ingestion toxicity ( ${ }^{3}$ vater) of fission-product isotopes accunulated at SURP (shipped 5 years after discharge)

\begin{tabular}{|c|c|c|c|c|c|}
\hline $\begin{array}{rr}H & 3 \\
R & 85 \\
R & 90 \\
Y & 90 \\
0 & 106 \\
\text { H } 106\end{array}$ & $\begin{array}{l}1.25 E \\
5.89 E \\
2.34 E \\
3.52 E \\
3.89 E \\
3.89 E \\
4.16 E \\
1.80 E \\
7.45 E \\
1.12 E \\
4.91 E \\
9.19 E \\
2.48 E \\
2.48 E \\
8.00 E \\
1.12 E \\
2.15 E \\
4.45 E \\
2.45 E\end{array}$ & $\begin{array}{l}09 \\
07 \\
15 \\
13 \\
12 \\
07 \\
05 \\
11 \\
10 \\
13 \\
13 \\
08 \\
12 \\
07 \\
11 \\
10 \\
12 \\
10 \\
15\end{array}$ & $\begin{array}{l}2.83 \mathrm{E} \\
1.33 \mathrm{E} \\
5.34 \mathrm{E} \\
8.02 \mathrm{E} \\
8.26 \mathrm{E} \\
8.26 \mathrm{E} \\
9.44 \mathrm{E} \\
4.11 \mathrm{E} \\
1.70 \mathrm{E} \\
2.55 \mathrm{E} \\
1.12 \mathrm{E} \\
2.10 \mathrm{E} \\
5.05 \mathrm{E} \\
5.05 \mathrm{E} \\
1.82 \mathrm{E} \\
2.58 \mathrm{~B} \\
4.88 \mathrm{E} \\
1.01 \mathrm{E} \\
5.57\end{array}$ & $\begin{array}{l}09 \\
08 \\
15 \\
13 \\
12 \\
07 \\
05 \\
11 \\
11 \\
13 \\
14 \\
05 \\
12 \\
07 \\
12 \\
10 \\
12 \\
11 \\
15\end{array}$ & 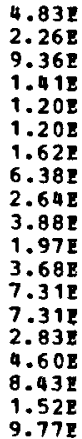 \\
\hline
\end{tabular}

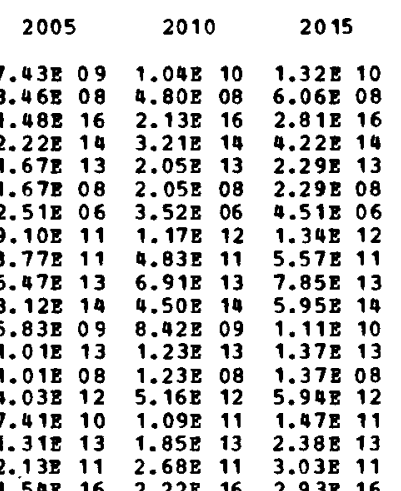

$$
2020
$$

2025

2030

2035

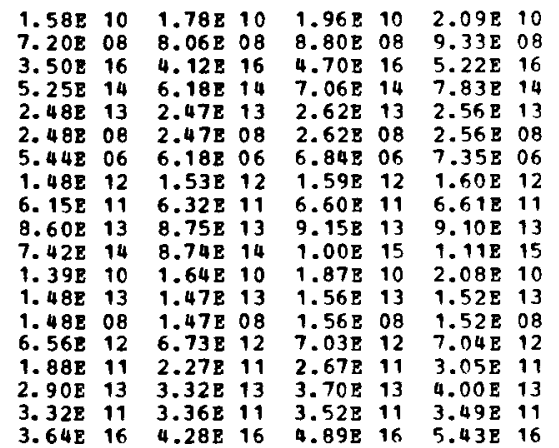

Table 3.37. Ingestion toxicity ( ${ }^{3}$ water) of heavy-elesent isotopes accunulated at sorPr (shipped 5 years after discharge)

\begin{tabular}{|c|c|c|c|c|c|c|c|c|c|c|c|c|c|c|c|c|c|c|}
\hline \multicolumn{2}{|c|}{1990} & \multicolumn{2}{|c|}{1995} & \multicolumn{2}{|l|}{2000} & \multicolumn{2}{|c|}{2005} & \multicolumn{2}{|l|}{2010} & 2015 & & \multicolumn{2}{|c|}{2020} & \multicolumn{2}{|c|}{2025} & \multicolumn{2}{|l|}{2030} & 2035 \\
\hline $\begin{array}{l}77 E \\
92 E \\
02 E \\
78 E \\
26 E \\
72 E \\
30 E \\
24 E \\
33 E \\
78 E \\
16 E \\
44 E \\
67 E\end{array}$ & $\begin{array}{l}08 \\
09 \\
12 \\
11 \\
12 \\
12 \\
c 9 \\
12 \\
10 \\
10 \\
09 \\
10 \\
12\end{array}$ & $\begin{array}{l}4.42 \mathrm{E} \\
1.15 \mathrm{E} \\
1.79 \mathrm{E} \\
2.91 \mathrm{E} \\
1.07 \mathrm{E} \\
9.90 \mathrm{E} \\
1.23 \mathrm{E} \\
3.05 \mathrm{E} \\
1.10 \mathrm{E} \\
6.84 \mathrm{E} \\
5.56 \mathrm{~B} \\
3.81 \mathrm{~B} \\
4.33 \mathrm{~B}\end{array}$ & $\begin{array}{l}09 \\
09 \\
13 \\
12 \\
12 \\
13 \\
09 \\
13 \\
10 \\
11 \\
05 \\
10 \\
12 \\
13\end{array}$ & $\begin{array}{l}1.99 \mathrm{E} \\
7.96 \mathrm{E} \\
2.06 \mathrm{E} \\
3.21 \mathrm{E} \\
5.23 \mathrm{I} \\
1.84 \mathrm{E} \\
1.78 \mathrm{E} \\
2.36 \mathrm{E} \\
5.45 \mathrm{E} \\
1.99 \mathrm{E} \\
1.16 \mathrm{E} \\
9.78 \mathrm{E} \\
6.61 \mathrm{E} \\
7.80 \mathrm{E}\end{array}$ & $\begin{array}{l}09 \\
09 \\
13 \\
12 \\
12 \\
13 \\
10 \\
13 \\
10 \\
11 \\
10 \\
10 \\
12\end{array}$ & & $\begin{array}{l}09 \\
10 \\
13 \\
12 \\
12 \\
13 \\
10 \\
13 \\
10 \\
11 \\
10 \\
11 \\
13 \\
14\end{array}$ & $\begin{array}{l}4.88 \mathrm{E} \\
1.92 \mathrm{E} \\
4.88 \mathrm{~B} \\
7.77 \mathrm{E} \\
1.26 \mathrm{E} \\
4.01 \mathrm{E} \\
4.31 \mathrm{E} \\
6.39 \mathrm{E} \\
1.30 \mathrm{E} \\
4.80 \mathrm{E} \\
2.58 \mathrm{E} \\
2.24 \mathrm{E} \\
1.46 \mathrm{E} \\
1.89 \mathrm{E}\end{array}$ & $\begin{array}{l}09 \\
10 \\
13 \\
12 \\
13 \\
13 \\
10 \\
13 \\
11 \\
11 \\
10 \\
11 \\
13 \\
14\end{array}$ & & $\begin{array}{l}09 \\
10 \\
13 \\
13 \\
13 \\
13 \\
10 \\
13 \\
11 \\
11 \\
10 \\
11 \\
13\end{array}$ & & $\begin{array}{l}09 \\
10 \\
13 \\
13 \\
13 \\
13 \\
10 \\
14 \\
11 \\
11 \\
10 \\
11 \\
13 \\
14\end{array}$ & & $\begin{array}{l}10 \\
10 \\
14 \\
13 \\
13 \\
13\end{array}$ & $\begin{array}{l}.31 \mathrm{E} \\
.99 \mathrm{E} \\
.20 \mathrm{E}\end{array}$ & $\begin{array}{l}10 \\
10 \\
14 \\
13\end{array}$ & 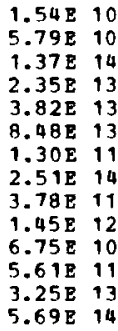 \\
\hline
\end{tabular}


Table 3.38. Inhalation toxicity $\left(\mathrm{a}^{3}\right.$ air) of light-elenent isotopes accunulated at SURF (shipped 5 years after discharge)

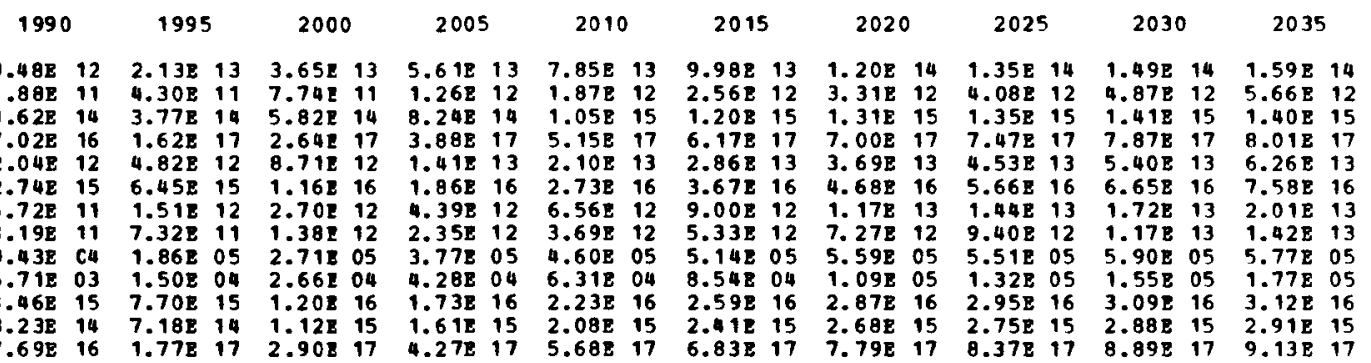

Table 3.39. Inhalation toxicity $\left(\mathrm{n}^{3}\right.$ air) of fission-product isotopes ccuaviated at soRP (shipped 5 years after discharge)

$\begin{array}{llllllllll}1990 & 1995 & 2000 & 2005 & 2010 & 2015 & 2020 & 2025 & 2030 & 2035\end{array}$

\begin{tabular}{rr} 
R & 3 \\
KR & 85 \\
\hline
\end{tabular}

SR 90

70
106

106

CD113

SE125

TE 125 H

$\operatorname{cs} 134$

$\operatorname{cs137}$

CA 1374

CR140

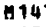

SH 151

20155

TOTAL

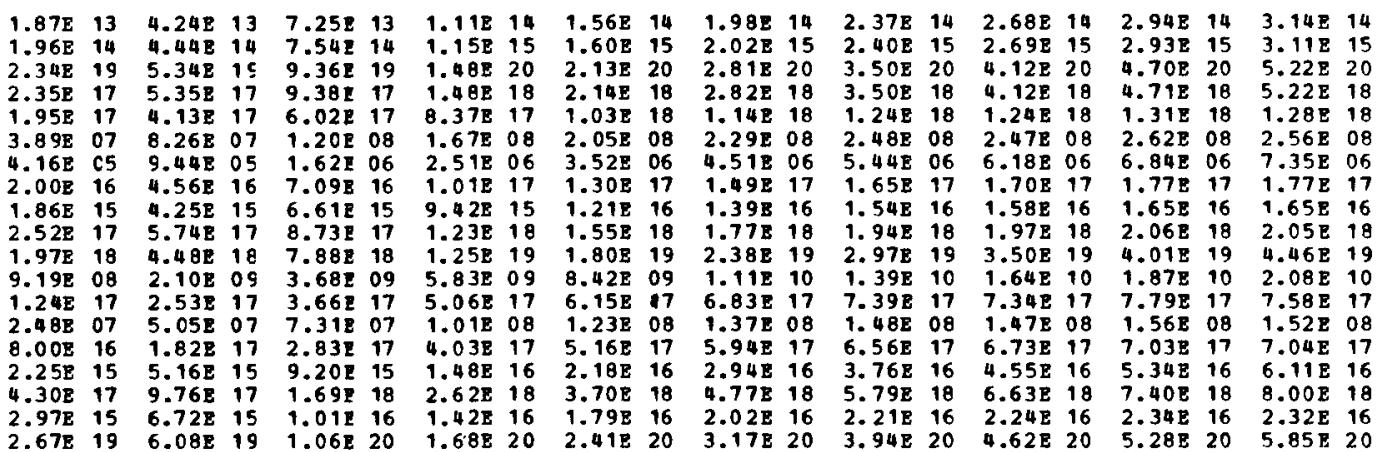

Table 3.40. Inhalation toricity $\left(a^{3}\right.$ air) of heary-elenent isotopes accuinulated suRF (shipped 5 years after discharge)

$\begin{array}{lllllllll}1990 & 1995 & 2000 & 2005 & 2010 & 2015 & 2020 & 2025 & 2030\end{array}$

0234

NF239

P0238

00239

PO24

P0242

An.241

A 4242 !

A 243

$\mathrm{CH} 242$

CH243

CA244

$\begin{array}{llllllllll}7.16 \mathrm{E} & 14 & 1.65 \mathrm{E} & 15 & 2.99 \mathrm{E} & 15 & 4.89 \mathrm{E} & 15 & 7.32 \mathrm{E} & 15 \\ 6.39 \mathrm{E} & 12 & 1.47 \mathrm{E} & 13 & 2.65 \mathrm{E} & 13 & 4.31 \mathrm{E} & 13 & 6.41 \mathrm{E} & 13\end{array}$

$\begin{array}{llllllllll}3.58 E & 20 & 8.24 E & 20 & 1.47 E & 21 & 2.37 E & 21 & 3.49 E & 21\end{array}$

$\begin{array}{llllllllll}6.48 E & 19 & 1.49 E & 20 & 2.68 \mathrm{E} & 20 & 4.35 E & 20 & 6.48 \mathrm{E} & 20\end{array}$

$\begin{array}{llllll}1.05 E & 20 & 2.42 \mathrm{E} & 20 & 4.36 \mathrm{E} & 20\end{array}$

$\begin{array}{llllll}3.15 E & 20 & 7.12 \mathrm{E} & 20 & 1.22 \mathrm{E} & 21\end{array}$

$\begin{array}{llllll}3.58 E & 17 & 8.25 \mathrm{E} & 17 & 1.49 \mathrm{E} & 18\end{array}$

$1.05 \mathrm{E} 20 \quad 2.47 \mathrm{E} 2 \mathrm{C} \quad 4.72 \mathrm{E} 20$

$2.67 \mathrm{E} 17$ 6.09E 17 T.09E 18

$1.56 E \quad 17$ 2.21E 18 3.98E 18

$1.58 \mathrm{E}$ 16 $3.42 \mathrm{E} 16 \quad 5.80 \mathrm{E} 16$

$\begin{array}{llllll}6.09 E & 17 & 1.39 \mathrm{E} & 18 & 2.45 \mathrm{E} & 18 \\ 3.90 \mathrm{E} & 19 & \mathbf{8 . 8 9 E} & 15 & 1.54 \mathrm{E} & 20\end{array}$

$7.08 \mathrm{E} 20 \mathrm{O} 1.05 \mathrm{E} 21$

$\begin{array}{llll}1.90 \mathrm{~B} & 21 & 2.67 \mathrm{E} & 21 \\ 2.41 \mathrm{~B} & 18 & 3.598 & 18\end{array}$

$8.11 \mathrm{E} 20 \mathrm{1} 2.28 \mathrm{E} 21$

$\begin{array}{lllll}1.768 & 18 & 2.61 \mathrm{E} & 18\end{array}$

$6.46 E 18 \quad 9.61818$

$9.06 \mathrm{E} \quad 16 \quad 1.29 \mathrm{E} \quad 17$

$3.88 \mathrm{E} \quad 18 \mathrm{5} \quad 5.61 \mathrm{E} \quad 18$

$1.01 \mathrm{E} 16$

1. $32 \mathrm{E} 16$

$1.63 \mathrm{E} 16$

$\begin{array}{llll}1.97 \mathrm{E} & 16 \quad 2.30 \mathrm{E} \quad 16\end{array}$

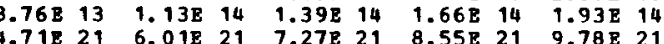
$8.86 \mathrm{E} 20 \quad 1.15 \mathrm{E} 21$ 1.41E 21 1.69E 21 1.96E 21

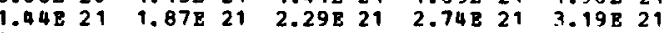

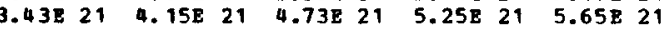
$\begin{array}{llllllllll}4.91 \mathrm{E} & 18 & 6.35 \mathrm{E} & 18 & 7.81 \mathrm{E} & 18 & 9.33 \mathrm{~B} & 18 & 1.08 \mathrm{E} & 19\end{array}$ $\begin{array}{llllllllll}1.86 \mathrm{E} & 21 & 2.54 \mathrm{E} & 21 & 3.31 \mathrm{E} & 21 & 4.13 \mathrm{~B} & 21 & 5.01 \mathrm{E} & 21\end{array}$ $3.54 \mathrm{E} 18$ 4.55E 18 5.54E $186.56 \mathrm{~B} 18 \mathrm{7} .56 \mathrm{E}$

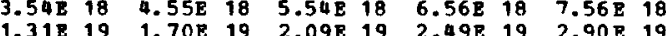
$1.31819 \quad 1.70 \mathrm{E} 1902.09 \mathrm{E} 19 \mathrm{2.49E} 19$ 2.90 E 19

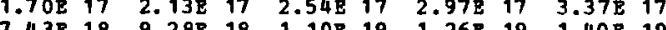

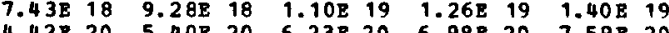

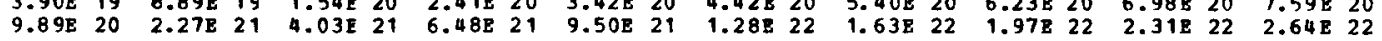


Table 3.41. Cories of accunulated 11ght-elenent 1sotopes after 2036

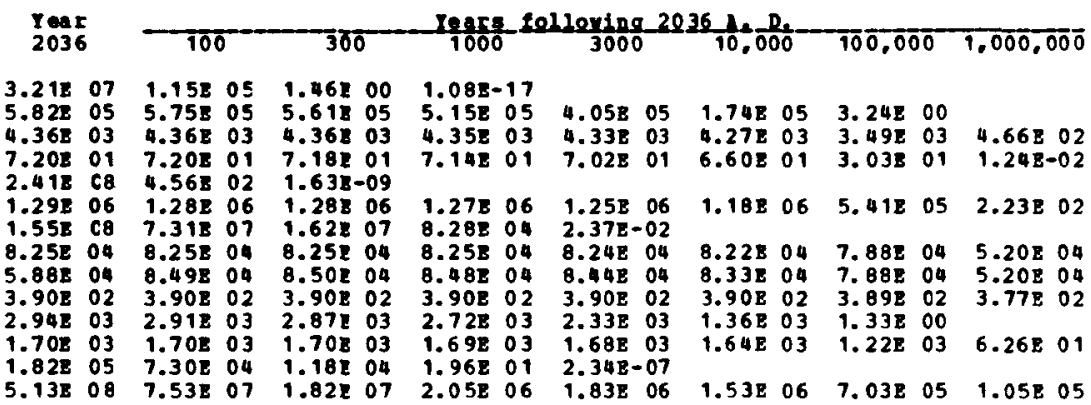

Table 3.42. Curies of accunalated fission-product isotopes after 2036

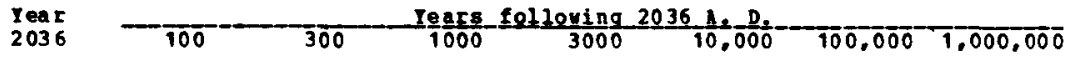

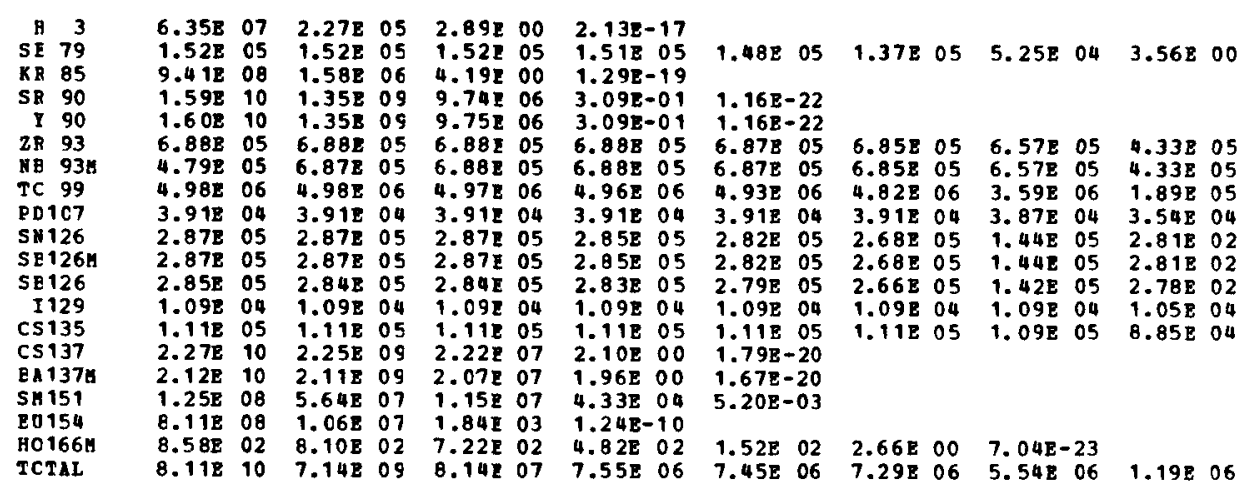


rable 3.43. Curies of accuanlated heary-element isotopes after 2036

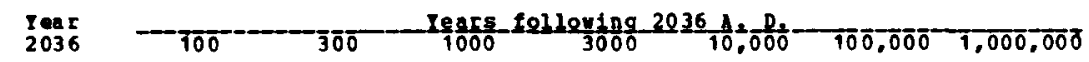

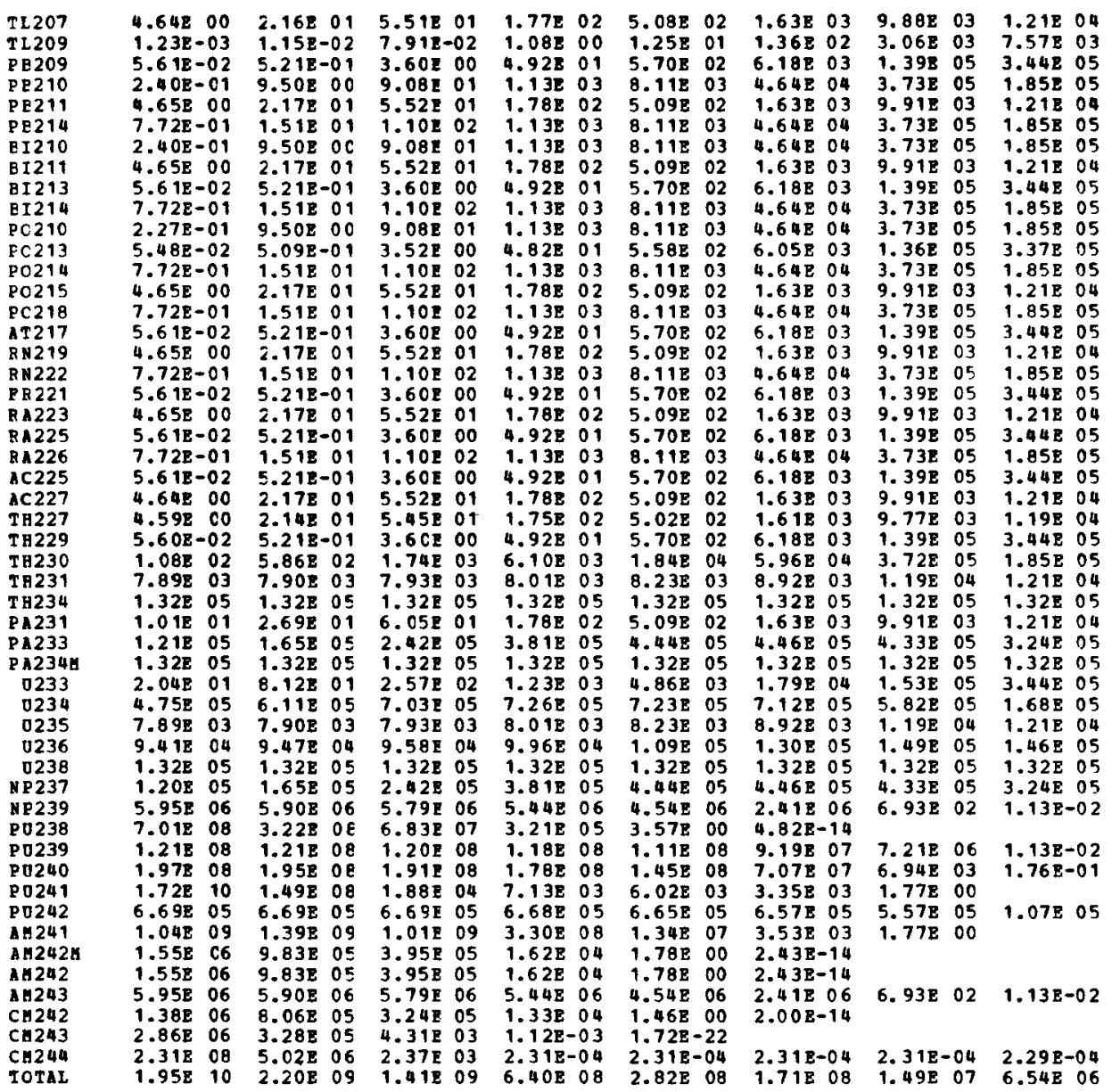


Table 3.44. Projected accunalation of spent fuel assenblies at sonfr (ahipped 10 years after discharge)

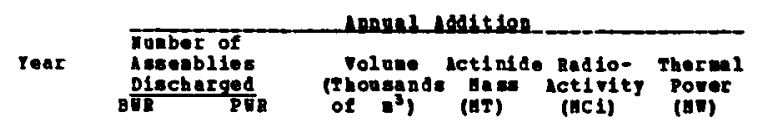

1989

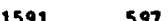

$\begin{array}{ll}1591 & 597 \\ 2027 & 955\end{array}$

1958

1988

1990

1991
1992

1993
1994
1995
1996

1997

1998
1999
2000

200

2002

2004

2005

2006

200

2009

2010

2012

2013

2015

2016

2018

2020

2021

2023

2023
2024
2025
2026

2025
2026
2027
2028

2028
2029
2030
2031

2032
2033

2034

2035
2036

2038

2040
2041

$\begin{array}{rr}0.25 & 549 \\ 0.33 & 742 \\ 0.41 & 918 \\ 0.48 & 1073 \\ 0.54 & 1222 \\ 0.68 & 1531 \\ 0.73 & 1649 \\ 0.78 & 1765\end{array}$

1765

$\begin{array}{ll}0.86 & 2192 \\ 0.97 & \end{array}$

$\begin{array}{ll}1.01 & 2297 \\ 1.17 & 2667\end{array}$

$1.33 \quad 3020$

$\begin{array}{ll}1.46 & 3322 \\ 1.59 & 3607\end{array}$

$\begin{array}{ll}1.72 & 3894 \\ 1.85 & 203\end{array}$

$\begin{array}{ll}1.85 & 203 \\ 2.00 & 4532\end{array}$

$\begin{array}{ll}2.00 & 4532 \\ 2.15 & 4875\end{array}$

$2.30 \quad 5227$

$\begin{array}{ll}2.47 & 5600 \\ 2.65 & 6005\end{array}$

$2.83 \quad 6417$

$\begin{array}{ll}3.01 & 6825 \\ 3.19 & 7227\end{array}$

$\begin{array}{ll}3.19 & 7227 \\ 3.36 & 7622\end{array}$

3.53798

$\begin{array}{ll}3.64 & 8256 \\ 3.74 & 8477\end{array}$

3.74
3.83
3.9665

$3.90 \quad 8820$

$3.98 \quad 9024$

4.06
$4.14 \quad 9368$
4.24

$\begin{array}{rr}.14 & 9368 \\ .24 & 9587 \\ .34 & 9802\end{array}$

. .34
$4.41 \quad 9802$
.4964

$\begin{array}{rr}4.41 & 9964 \\ .50 & 10178\end{array}$

$4.55 \quad 10301$
. .55

$\begin{array}{rr}4.65 & 10519 \\ 4.72 \quad 10654\end{array}$

$4.62 \quad 1045$

4.61
.610454

$\begin{array}{rr}.650 & 10424 \\ 4.57 & 10346\end{array}$

$\begin{array}{ll}4.57 & 10346 \\ 4.61 & 1042\end{array}$

$\begin{array}{ll}4.61 & 10428\end{array}$

$\begin{array}{rr}4.74 & 10740 \\ 4.85 & 10976\end{array}$

$\begin{array}{ll}0.85 & 10976 \\ . .92 & 11135 \\ 5.01 & 11331 \\ 4.95 & 11107\end{array}$

$\begin{array}{lll}4.95 & 11187 & 3987 \\ 4.85 & 10944 & 3908\end{array}$

4.82 10880

$\begin{array}{ll}4.82 & 10880 \\ 4.85 & 10950\end{array}$

87
135
187
307
375
497
556
604
662
737
768
804
1010
1133
1248
1356
1965
1579
1700
1826
1958
2099
2246
2397
2546
2696
2834
2956
3062
3141
3201
3276
3340
3403
3480
3552
3608
3678
3724
3792
3829
3772
3778
3770
3743
3769
3788
3871
3942
3985
4037
3987
3908
3879
3881
3901
3914

0
0
1
1
1
2
2
2
2
2
2
3
3
3
4
4
4
5
5
6
6
6
7
7
8
8
9
9
9
10
10
10
10
10
11
11
11
11
11
12
12
11
12
11
11
11
12
12
12
12
12
12
12
12
12
12
12

TIHE AFTER SHOTDORH, YEARS

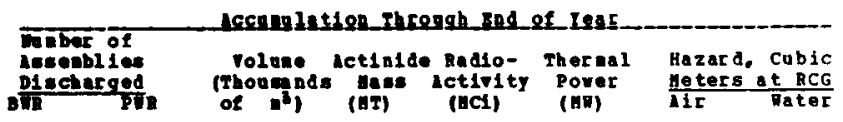

1591
3617

597
1452

$\begin{array}{rr}3617 & 1452 \\ 3575 & 2726 \\ 9231 & 9066 \\ 11960 & 5696\end{array}$

11006

14320

17820

25508

$29793 \quad 18572$

39282

21964

25916
30391

504
5140

35300

58356

40613

$74105 \quad 52486$

$\begin{array}{ll}02978 & 59107 \\ 92557 & 66217\end{array}$

$\begin{array}{rr}92557 & 66217 \\ 102862 & 73926\end{array}$

11393881968

12585490673

$\begin{array}{rr}138627 & 99965 \\ 152245 & 109834\end{array}$

$\begin{array}{ll}152245 & 109834 \\ 166711 & 120268\end{array}$

102015

120268
131253

198093142746

(1)

154617

249422

267287

166786
179216

19216

285525
304130
218005

323090238005

342764231449

$363227 \quad 258904$

383965

05242

258904
272970

287302
302037

331848

492245
546593
513213
361666

$533956 \quad 376763$

$555233 \quad 391471$

576958 406184

598610
520547
636371

620547
642966
6452036

642966
666144
6657754

$689988 \quad 483646$

$\begin{array}{ll}713679 & 499275 \\ 736992 & 514511\end{array}$

760188529605

$783511 \quad 544693$

807000
859873
830502 575120

$\begin{array}{ll}0.2 & \\ 0.5 & 1 \\ 1.0 & 2 \\ 1.5 & 3 \\ 2.0 & \\ 2.7 & 6\end{array}$

$\begin{array}{rr}0.2 & 519 \\ 0.6 & 129 \\ 1.0 & 220 \\ 1.5 & 328 \\ 2.0 & 450\end{array}$

$2.7 \quad 6035$

3.4
5.2 948

$\begin{array}{ll}5.1 & 11393 \\ 6.0 & 13585 \\ 7.0 & 15802\end{array}$

$\begin{array}{ll}6.0 & 13589 \\ 7.0 & 15809\end{array}$

$\begin{array}{rr}8.2 & 18549 \\ 9.5 & 21569\end{array}$

$\begin{array}{ll}11.0 & 2489 \\ 12.6 & 28998\end{array}$

$\begin{array}{ll}12.6 & 28498 \\ 14.3 & 32392\end{array}$

$16.2 \quad 36595$

$\begin{array}{ll}18.2 & 41127 \\ 20.3 & 46002\end{array}$

$22.6 \quad 51229$

25.1
27.7

$\begin{array}{ll}27.7 & 62839 \\ 30.6 & 69251 \\ 33.6 & 76076\end{array}$

$\begin{array}{ll}33.6 & 76076 \\ 36.8 & 83303\end{array}$

36.8
$40.1 \quad 90926$

43.79891

47.3107169

$51.0 \quad 115646$

$58.8 \quad 133132$

$62.7 \quad 142152$

$\begin{array}{ll}66.8 & 15134 \\ 70.9 & 160714\end{array}$

$\begin{array}{ll}75.2 & 170301 \\ 79.5 & 180103\end{array}$

83.9190067

$88.4 \quad 200245$

$93.0 \quad 210546$

97.6221065

102.3231719

$107.0 \quad 242170$

$111.6 \quad 252624$

116.2263048

$120.8 \quad 273394$

135.4283822

$134.7 \quad 305042$

$\begin{array}{ll}139.6 & 316017 \\ 144.5 & 327152\end{array}$

$144.5 \quad 327152$

$154.5 \quad 349670$

159.3360614

164.1371473

$\begin{array}{rr}169.0 & 382353 \\ 173.8 & 393303\end{array}$

$178.7 \quad 404303$

87

87
219
398
591

691
1042
1502

1502

2500

3115

3746

5125

5964

7920

9016
10185

10185
11432

11432
12760

14173
15675
1725

17272

18966
20759

2264

24623

26897

30957

33131

37483

39665

41848

44046
46254

48454

50662

52855
55053
57227

57227

59284
61292

63237

66948

68762

70611

74345
76210

76210
77975

77975

8118

82716
84229
85717

85717

$\begin{array}{ll}830602 & 575120 \\ 830602 & 575120\end{array}$

830602 575120

830602575120

(830602

830602
830602

830602

830602
830602

575120

575120
575120

575120
575120
$178.7 \quad 404303$

$178.7 \quad 404303$

$178.7 \quad 404303$

178.7904303

178.7404303
178.7404303

178.7404303
8566
1489
647
291
180
75
21
13
8

$2.1 \mathrm{E} 19 \quad 4.8 \mathrm{z} 13$ 9.381922 .2814 1.6220
2.4820 3.5E 20 8.2E 14 4. 72 20 1.1815 6.08 20 1.4E 15 $7.4820 \quad 1.7815$ 8.92 202021215 1.22 212.9815 $1.4 \mathrm{E} 21 \quad 3.3 \mathrm{E} 15$ $1.7821 \quad 3.8 \mathrm{P} 15$ 1.9221 4. 4215 2.28 215.0815 $\begin{array}{llll}2.5 \mathrm{E} & 21 & 5.6 \mathrm{E} & 15 \\ 2.8 \mathrm{E} & 21 & 6.3 \mathrm{E} & 15 \\ 3 & & \end{array}$ $\begin{array}{llll}2.8 E & 21 & 6.3 E & 15 \\ 3.2 E & 21 & 7.1 E & 15\end{array}$ 3.58 217.9815 3.98 218.7815 4.32219 .6815 $4.8 \mathrm{E} 21.1 \mathrm{E} 16$ 5. $8 E 21 \quad 1.38$ 16 5. 3821 1.4F 6. 7.0E 21 1.58 16 8.6E $21, .9816$ 9.2E $21 \quad 20 \mathrm{E} 16$ $9.9 \mathrm{E} 212.1816$ $1.0 \mathrm{~B} 22 \quad 2.2816$ I.1E $22 \quad 22 \mathrm{E} 16$ 1.28222 .5816 1.2E 22 2.68 16 $1.3 \mathrm{~B} 22 \quad 2.7 \mathrm{E} 16$ 1. $4 \mathrm{E} 222.9 \mathrm{E} 16$ $1.5 E^{22} \quad 3.1 \mathrm{E} 96$ $1.6 \mathrm{E} \mathrm{22} 3.2 \mathrm{E} 16$ $1.7822 \quad 3.4 \mathrm{E} 16$ 1.78 $22 \quad 3.5 \mathrm{~B} 16$ $\begin{array}{llll}1.88 & 22 & 3.68 & 16 \\ 1.98 & 22 & 3.78 & 6\end{array}$ $1.9 \mathrm{E} 22 \quad 3.8 \mathrm{E} 16$ 2. OE 22 3.9E 16 $2.0 \mathrm{E} 22 \quad 4.0 \mathrm{~B} 16$ $2.1 \mathrm{E} 22 \quad \mathrm{AE} \quad 1 \mathrm{~K}$ 2.2E 22 4. 3816 $2.3 \mathrm{E} 22$ प. $4 \mathrm{E} 16$ 2.3E 22 4.5816 2.4822 ..68 16 2.5822 प.78 16 $2.6 \mathrm{~B} 22 \quad 4.8 \mathrm{E} \quad 16$ 
Table 3.45. Grans of light elenents accunulated at soRF (shipped 10 years after discharge)

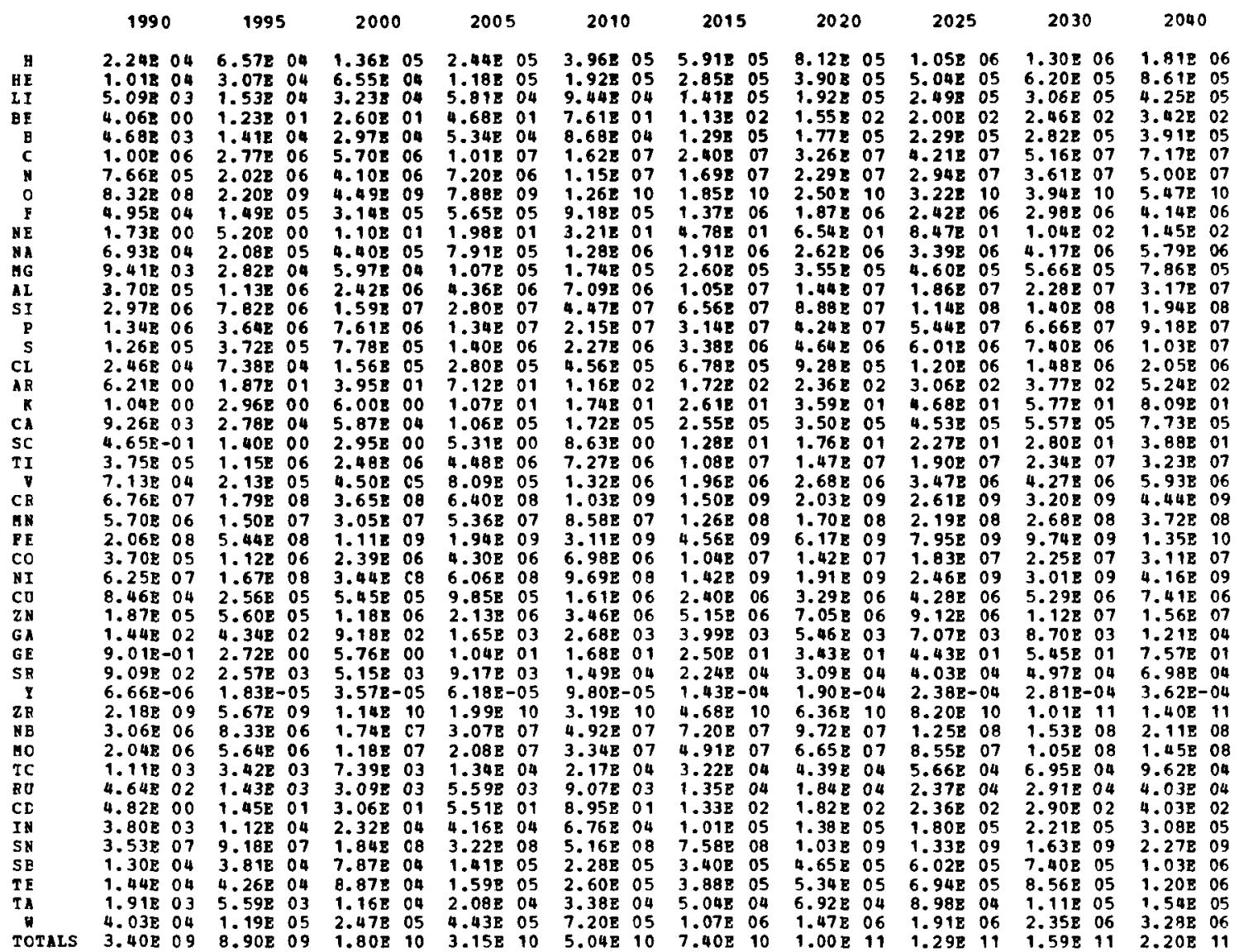


Table 3.46. Grass of flselon-product elenents accunulated at surp (shipped 10 years after discharge) $\begin{array}{llllllllll}1990 & 1995 & 2000 & 2005 & 2010 & 2015 & 2020 & 2025 & 2030 & 2040\end{array}$

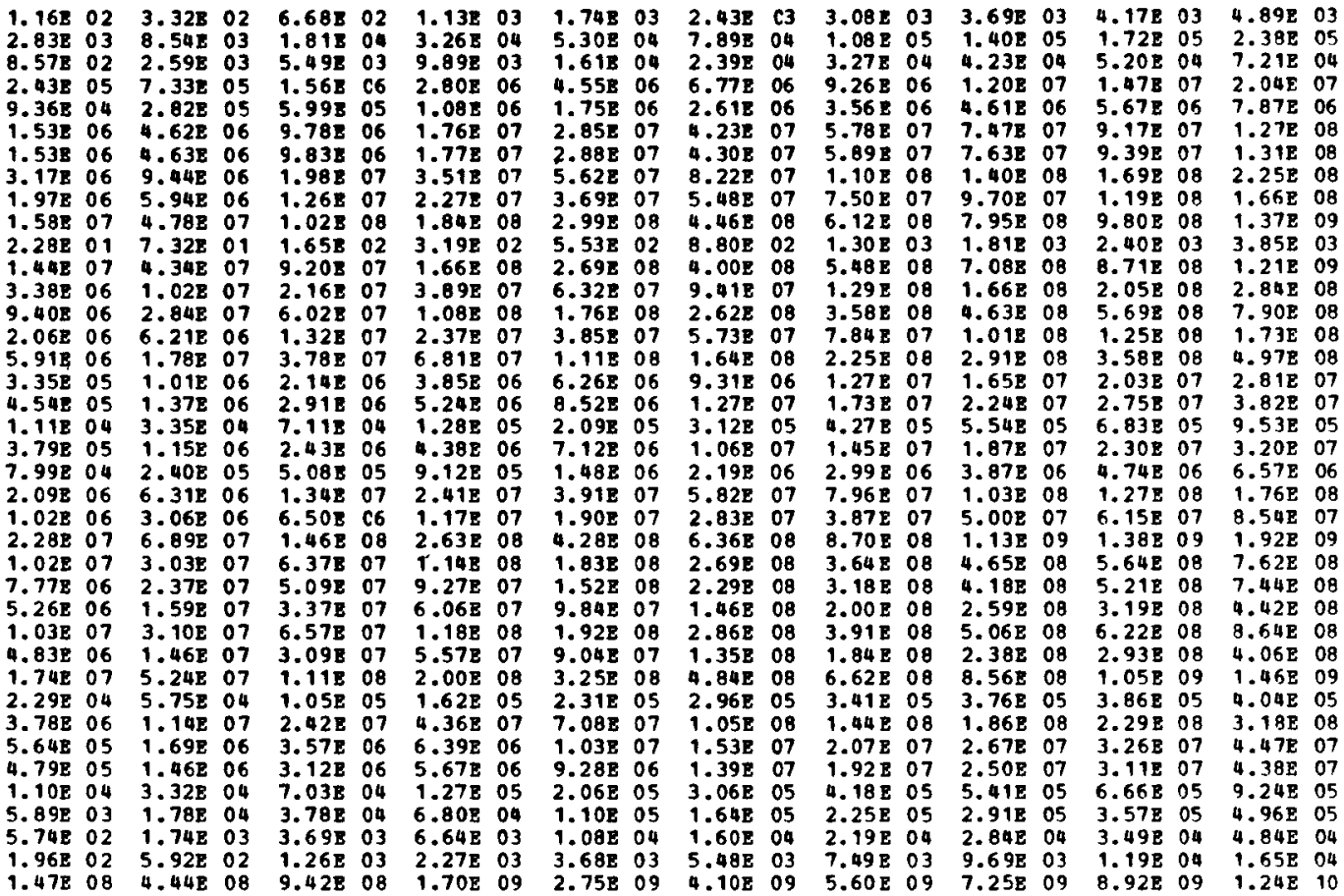

Table 3.47. Grams of heary elements accunulated at SoRPP (shipped 10 years after discharge)

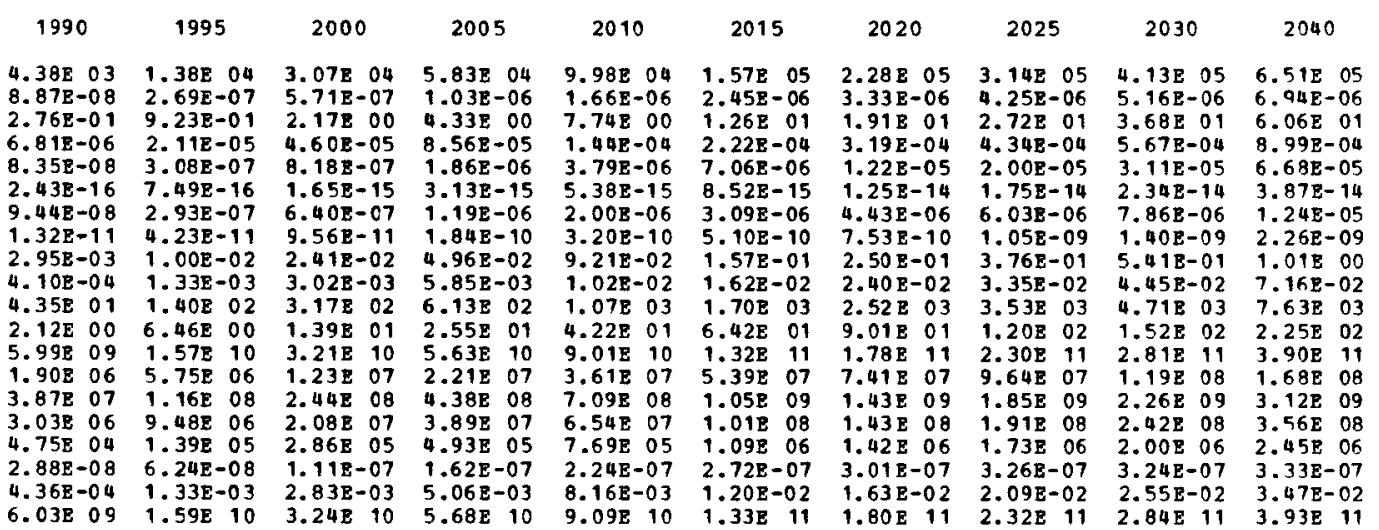


Table 3.48. Curies of 11ght-elenent 180topes accunulated at sorfF (shipped 10 years after discharge) $\begin{array}{llllllllll}1990 & 1995 & 2000 & 2005 & 2010 & 2015 & 2020 & 2025 & 2030 & 2040\end{array}$

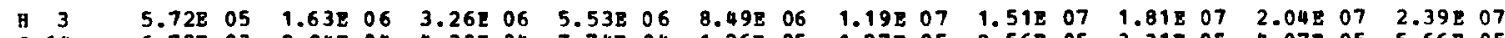

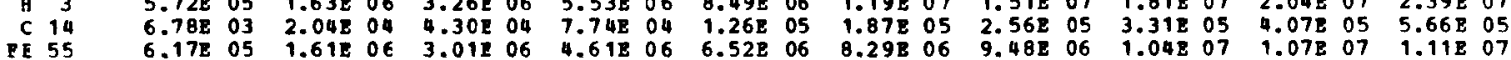

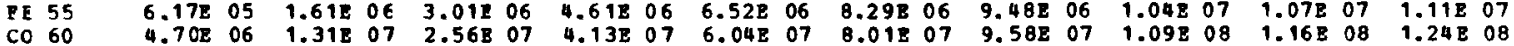

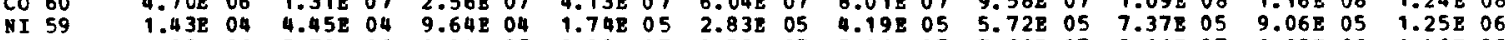

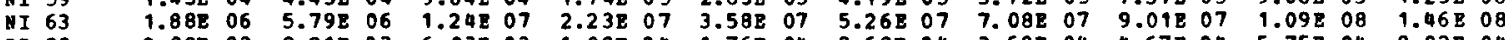

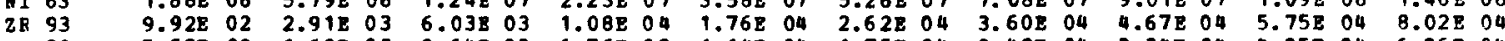

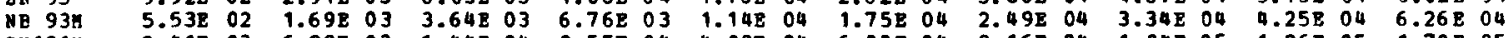
$\begin{array}{llllllllllllllllllllll}5 N 121 M & 2.41 E & 03 & 6.99 E & 03 & 1.44 E & 04 & 2.55 E & 04 & 4.09 E & 04 & 6.03 E & 04 & 8.16 E & 04 & 1.04 E & 05 & 1.268 & 05 & 1.70 \mathrm{E} & 05\end{array}$

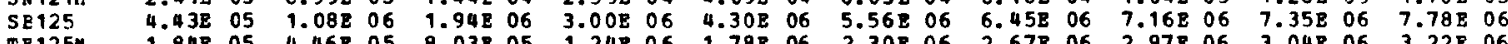

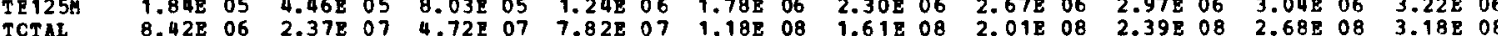

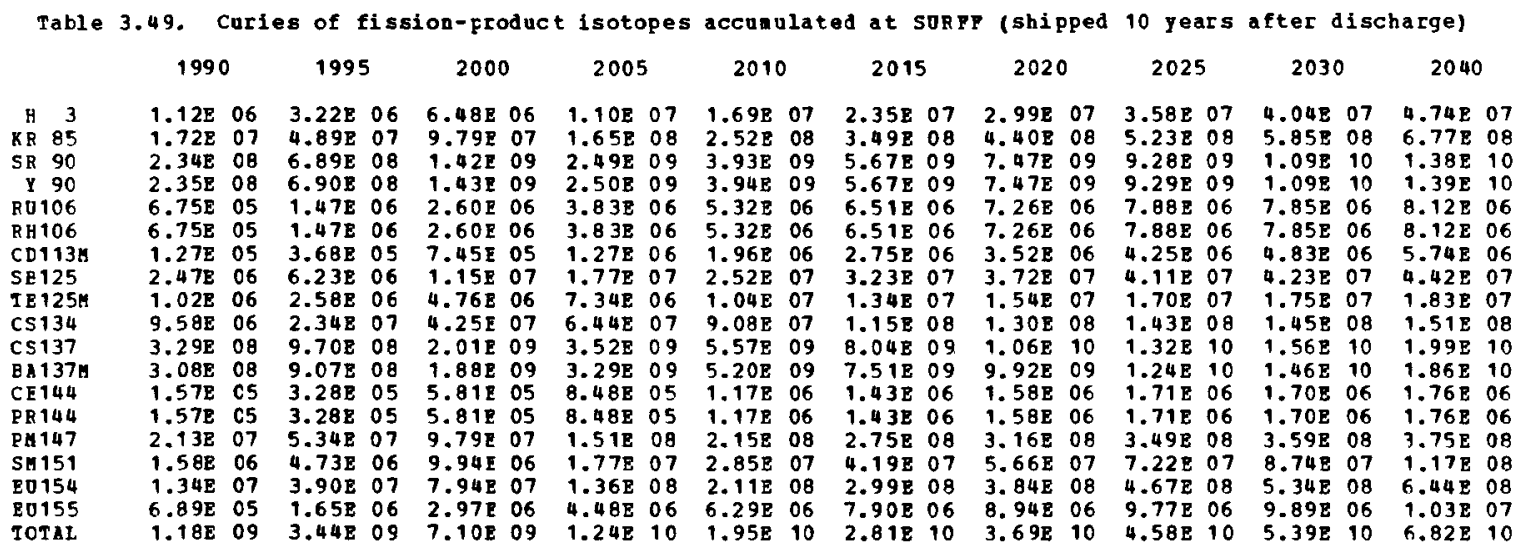

Table 3.50. Curies of heavy-elenent isotopes accunulated at surpf (shipped 10 years after discharge)

\begin{tabular}{|c|c|c|c|c|c|c|c|c|c|c|c|c|c|c|c|c|c|c|}
\hline & \multicolumn{2}{|c|}{1995} & \multicolumn{2}{|c|}{2000} & \multicolumn{2}{|l|}{2005} & \multicolumn{2}{|l|}{2010} & \multicolumn{2}{|l|}{2015} & \multicolumn{2}{|l|}{2020} & 2025 & & \multicolumn{2}{|l|}{2030} & 2040 \\
\hline $\begin{array}{l}24 E \\
89 E \\
80 E \\
41 E \\
28 E \\
92 E \\
74 E \\
15 E \\
91 E \\
87 E \\
57 E \\
10 E \\
71 E \\
18 E\end{array}$ & $\begin{array}{l}03 \\
04 \\
06 \\
06 \\
06 \\
08 \\
03 \\
06 \\
04 \\
04 \\
04 \\
04 \\
06 \\
08\end{array}$ & $\begin{array}{l}1.59 \mathrm{E} \\
2.08 \mathrm{E} \\
2.64 \mathrm{E} \\
4.23 \mathrm{E} \\
6.86 \mathrm{E} \\
8.42 \mathrm{E} \\
2.33 \mathrm{E} \\
2.88 \mathrm{E} \\
5.68 \mathrm{E} \\
2.08 \mathrm{E} \\
4.67 \mathrm{E} \\
1.21 \mathrm{E} \\
1 . \mathrm{CBE} \\
9.20 \mathrm{E}\end{array}$ & $\begin{array}{l}04 \\
05 \\
07 \\
06 \\
06 \\
08 \\
04 \\
07 \\
04 \\
05 \\
04 \\
05 \\
07 \\
08\end{array}$ & $\begin{array}{l}3.38 \mathrm{E} \\
4.42 \mathrm{E} \\
5.56 \mathrm{E} \\
8.93 \mathrm{E} \\
1.45 \mathrm{E} \\
1.70 \mathrm{E} \\
4.95 \mathrm{E} \\
6.35 \mathrm{E} \\
1.19 \mathrm{E} \\
4.42 \mathrm{E} \\
9.80 \mathrm{E} \\
2.51 \mathrm{E} \\
2.22 \mathrm{E} \\
1.87 \mathrm{E}\end{array}$ & $\begin{array}{l}04 \\
05 \\
07 \\
06 \\
07 \\
09 \\
04 \\
07 \\
05 \\
05 \\
04 \\
05 \\
07 \\
09\end{array}$ & $\begin{array}{l}6.12 \mathrm{E} \\
7.96 \mathrm{E} \\
9.93 \mathrm{E} \\
1.61 \mathrm{~B} \\
2.62 \mathrm{E} \\
2.91 \mathrm{E} \\
8.92 \mathrm{E} \\
1.19 \mathrm{E} \\
2.13 \mathrm{E} \\
7.95 \mathrm{~B} \\
1.75 \mathrm{E} \\
4.40 \mathrm{E} \\
3.84 \mathrm{E} \\
3.21 \mathrm{E}\end{array}$ & $\begin{array}{l}04 \\
05 \\
07 \\
07 \\
07 \\
09 \\
04 \\
08 \\
05 \\
05 \\
05 \\
05 \\
07 \\
09\end{array}$ & $\begin{array}{l}1.00 \mathrm{~B} \\
1.29 \mathrm{~B} \\
1.60 \mathrm{E} \\
2.61 \mathrm{E} \\
4.25 \mathrm{E} \\
4.50 \mathrm{~B} \\
1.45 \mathrm{E} \\
2.01 \mathrm{E} \\
3.45 \mathrm{E} \\
1.29 \mathrm{E} \\
2.83 \mathrm{E} \\
6.97 \mathrm{E} \\
5.97 \mathrm{E} \\
4.99 \mathrm{E}\end{array}$ & $\begin{array}{l}05 \\
06 \\
08 \\
07 \\
07 \\
09 \\
05 \\
08 \\
05 \\
06 \\
05 \\
05 \\
07 \\
09\end{array}$ & $\begin{array}{l}1.50 \mathrm{E} \\
1.92 \mathrm{E} \\
2.35 \mathrm{E} \\
3.89 \mathrm{E} \\
6.32 \mathrm{E} \\
6.33 \mathrm{E} \\
2.15 \mathrm{E} \\
3.10 \mathrm{E} \\
5.10 \mathrm{E} \\
1.92 \mathrm{E} \\
4.19 \mathrm{E} \\
1.01 \mathrm{E} \\
8.48 \mathrm{E} \\
7.07 \mathrm{E}\end{array}$ & $\begin{array}{l}05 \\
06 \\
08 \\
07 \\
07 \\
09 \\
05 \\
08 \\
05 \\
06 \\
05 \\
06 \\
07 \\
09\end{array}$ & $\begin{array}{l}2.06 \mathrm{E} \\
2.63 \mathrm{E} \\
3.17 \mathrm{E} \\
5.32 \mathrm{E} \\
8.65 \mathrm{E} \\
8.12 \mathrm{E} \\
2.95 \mathrm{E} \\
4.42 \mathrm{E} \\
6.93 \mathrm{E} \\
2.63 \mathrm{E} \\
5.69 \mathrm{E} \\
1.33 \mathrm{E} \\
1.10 \mathrm{E} \\
9.14 \mathrm{E}\end{array}$ & $\begin{array}{l}05 \\
06 \\
08 \\
07 \\
07 \\
09 \\
05 \\
08 \\
05 \\
06 \\
05 \\
06 \\
08 \\
09\end{array}$ & $\begin{array}{l}2.69 \mathrm{E} \\
3.40 \mathrm{E} \\
4.05 \mathrm{E} \\
6.8 \mathrm{E} \\
1.12 \mathrm{E} \\
9.82 \mathrm{E} \\
3.81 \mathrm{E} \\
5.93 \mathrm{E} \\
8.89 \mathrm{E} \\
3.40 \mathrm{E} \\
7.31 \mathrm{E} \\
1.67 \mathrm{E} \\
1.34 \mathrm{E} \\
1.11 \mathrm{E}\end{array}$ & $\begin{array}{l}05 \\
06 \\
08 \\
07 \\
08 \\
09 \\
05 \\
08 \\
05 \\
06 \\
05 \\
06 \\
08 \\
10\end{array}$ & $\begin{array}{l}3.33 \mathrm{~B} \\
4.17 \mathrm{E} \\
4.90 \mathrm{E} \\
8.46 \mathrm{~B} \\
1.38 \mathrm{E} \\
1.12 \mathrm{E} \\
4.69 \mathrm{E} \\
7.56 \mathrm{~B} \\
1.08 \mathrm{E} \\
4.17 \mathrm{E} \\
8.90 \mathrm{~B} \\
1.97 \mathrm{~B} \\
1.54 \mathrm{~B} \\
1.28 \mathrm{~B}\end{array}$ & $\begin{array}{l}05 \\
06 \\
08 \\
07 \\
08 \\
10 \\
05 \\
08 \\
06 \\
06 \\
05 \\
06 \\
08 \\
10\end{array}$ & $\begin{array}{l}4.70 \mathrm{E} \\
5.79 \mathrm{E} \\
6.58 \mathrm{E} \\
1.18 \mathrm{E} \\
1.91 \mathrm{E} \\
1.34 \mathrm{E} \\
6.51 \mathrm{E} \\
1.12 \mathrm{E} \\
1.48 \mathrm{E} \\
5.79 \mathrm{E} \\
1.21 \mathrm{E} \\
2.52 \mathrm{E} \\
1.88 \mathrm{E} \\
1.57 \mathrm{E}\end{array}$ \\
\hline
\end{tabular}


Table 3.51. Matts of light-eleaent isotopes accunulated at sorrp (shipped 10 years after discharge)

\begin{tabular}{|c|c|c|c|c|c|c|c|c|c|c|c|c|c|c|c|}
\hline & 1990 & 1995 & 2000 & 2005 & & 201 & & 201 & & $202 \mathrm{C}$ & & 2025 & 203 & & 2040 \\
\hline $\begin{array}{ll}\text { H } & 3 \\
\text { C } & 14 \\
\text { FE } & 55 \\
\text { CO } & 60 \\
\text { II } & 63 \\
\text { ZR } & 93 \\
\text { HB } & 934 \\
\text { SH } 1214 \\
\text { SB } 125 \\
\text { TE125M } \\
\text { TOTAL }\end{array}$ & $\begin{array}{cc}2.03 E & 01 \\
2.01 E & 00 \\
8.05 E & 02 \\
7.35 E & 04 \\
3.01 E & 02 \\
1.18 E-01 \\
1.93 E-01 \\
2.52 E & 00 \\
1.80 E & 03 \\
1.58 E & 02 \\
7.66 E & 04\end{array}$ & 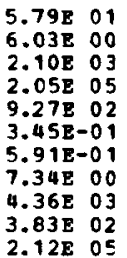 & $\begin{array}{lll}1.16 \mathrm{E} & 02 \\
1.28 \mathrm{E} & 01 \\
3.93 \mathrm{E} & 03 \\
4.00 \mathrm{E} & 05 \\
1.99 \mathrm{E} & 03 \\
7.15 \mathrm{E} & 01 \\
1.27 \mathrm{E} & 00 \\
1.51 \mathrm{E} & 01 \\
7.85 \mathrm{E} & 03 \\
6.90 \mathrm{E} & 02 \\
4.14 \mathrm{E} & 05\end{array}$ & $\begin{array}{l}1.97 E \\
2.29 E \\
6.02 E \\
6.45 E \\
3.57 E \\
1.28 E \\
2.37 E \\
2.67 E \\
1.22 E \\
1.07 E \\
6.68 E\end{array}$ & $\begin{array}{ll}02 & 2 \\
01 & 1 \\
03 & 3 \\
0 & 5 \\
0 & 3 \\
0 & 0 \\
0 & 0 \\
0 & 1 \\
04 & 4 \\
0 & 3 \\
0 & 5\end{array}$ & $\begin{array}{l}3.02 \mathrm{E} \\
3.73 \mathrm{E} \\
8.50 \mathrm{E} \\
9.44 \mathrm{E} \\
5.73 \mathrm{E} \\
2.08 \mathrm{E} \\
3.97 \mathrm{E} \\
4.30 \mathrm{E} \\
1.75 \mathrm{E} \\
1.53 \mathrm{E} \\
9.78 \mathrm{E}\end{array}$ & $\begin{array}{l}02 \\
01 \\
03 \\
05 \\
03 \\
00 \\
00 \\
01 \\
04 \\
03 \\
05\end{array}$ & $\begin{array}{l}4.22 E \\
5.55 \mathrm{E} \\
1.08 \mathrm{E} \\
1.25 \mathrm{E} \\
8.41 \mathrm{E} \\
3.11 \mathrm{E} \\
6.12 \mathrm{E} \\
6.33 \mathrm{E} \\
2.25 \mathrm{E} \\
1.98 \mathrm{E} \\
1.30 \mathrm{~B}\end{array}$ & $\begin{array}{l}02 \\
01 \\
04 \\
06 \\
03 \\
00 \\
00 \\
01 \\
04 \\
03 \\
06\end{array}$ & $\begin{array}{l}5.36 \mathrm{E} \\
7.59 \mathrm{E} \\
1.24 \mathrm{E} \\
1.50 \mathrm{E} \\
1.13 \mathrm{E} \\
4.27 \mathrm{E} \\
8.70 \mathrm{E} \\
8.56 \mathrm{E} \\
2.61 \mathrm{E} \\
2.30 \mathrm{E} \\
1.55 \mathrm{E}\end{array}$ & $\begin{array}{l}02 \\
01 \\
04 \\
06 \\
04 \\
00 \\
00 \\
01 \\
04 \\
03 \\
06\end{array}$ & 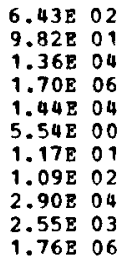 & $\begin{array}{l}7.25 \mathrm{E} \\
1.21 \mathrm{E} \\
1.40 \mathrm{E} \\
1.81 \mathrm{E} \\
1.74 \mathrm{E} \\
6.82 \mathrm{E} \\
1.49 \mathrm{E} \\
1.32 \mathrm{E} \\
2.98 \mathrm{E} \\
2.62 \mathrm{E} \\
1.88 \mathrm{E}\end{array}$ & $\begin{array}{l}02 \\
02 \\
04 \\
06 \\
04 \\
00 \\
01 \\
02 \\
04 \\
03 \\
06\end{array}$ & $\begin{array}{lll}8.52 \mathrm{E} & 0 \\
1.68 \mathrm{E} & 0 \\
1.44 \mathrm{E} & 0 \\
1.94 \mathrm{E} & 0 \\
2.34 \mathrm{E} & 0 \\
9.51 \mathrm{E} & 0 \\
2.19 \mathrm{E} & 0 \\
1.78 \mathrm{E} & 0 \\
3.15 \mathrm{E} & 0 \\
2.77 \mathrm{E} & 0 \\
2.02 \mathrm{E} & 0\end{array}$ \\
\hline
\end{tabular}

Table 3.52. Watts of fission-product isotopes accunulated at suRPP (shipped 10 years after discharge)

$\begin{array}{llllllllll}1990 & 1995 & 2000 & 2005 & 2010 & 2015 & 2020 & 2025 & 2030 & 2040\end{array}$

$\begin{array}{lllllllllllllllllllllllll}4.00 \mathrm{E} & 01 & 1.15 \mathrm{E} & 02 & 2.30 \mathrm{E} & 02 & 3.91 \mathrm{E} & 02 & 6.00 \mathrm{E} & 02 & \mathbf{8 . 3 7 E} & 02 & 1.06 \mathrm{E} & 03 & 1.27 \mathrm{E} & 03 & 1.44 \mathrm{E} & 03 & 1.69 \mathrm{E} & 03\end{array}$

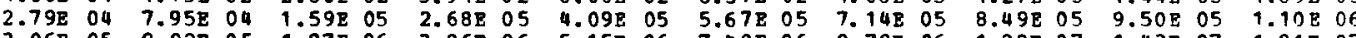
$\begin{array}{llllllllllllllllllll}3.06 \mathrm{E} & 05 & 9.02 \mathrm{E} & 05 & 1.87 \mathrm{E} & 06 & 3.26 \mathrm{E} & 06 & 5.15 \mathrm{E} & 06 & \mathbf{7 . 4 2 E} & 06 & 9.78 \mathrm{E} & 06 & 1.22 \mathrm{E} & 07 & 1.43 \mathrm{E} & 07 & 1.81 \mathrm{E} & 07\end{array}$

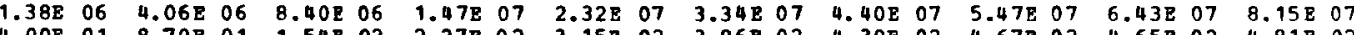

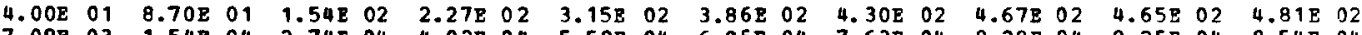

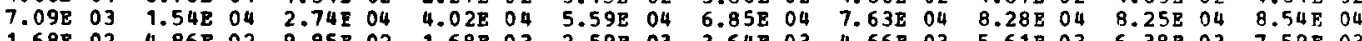

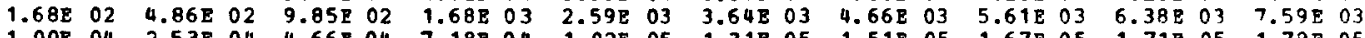

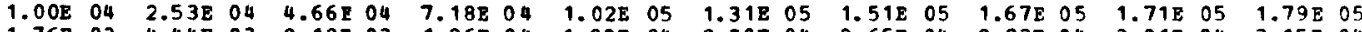
$\begin{array}{llllllllllllllllllll}1.76 \mathrm{E} & 03 & 4.44 \mathrm{E} & 03 & 8.18 \mathrm{E} & 03 & 1.26 \mathrm{E} & 04 & 1.80 \mathrm{E} & 04 & 2.30 \mathrm{E} & 04 & 2.65 \mathrm{E} & 04 & 2.93 \mathrm{E} & 04 & 3.01 \mathrm{E} & 04 & 3.15 \mathrm{E} & 04\end{array}$ $\begin{array}{llllllllllllllllllllll}1.01 E & 05 & 2.48 E & 05 & 4.50 E & 05 & 6.82 E & 05 & 9.62 E & 05 & 1.21 E & 06 & 1.38 E & 06 & 1.51 E & 06 & 1.54 E & 06 & 1.60 E & 06\end{array}$ $\begin{array}{lllllllllllllllllllllll}5.38 \mathrm{E} & 05 & 1.59 \mathrm{E} & 06 & 3.29 \mathrm{P} & 06 & 5.76 \mathrm{E} & 06 & 9.11 \mathrm{E} & 06 & 1.31 \mathrm{E} & 07 & 1.74 \mathrm{E} & 07 & 2.16 \mathrm{E} & 07 & 2.55 \mathrm{E} & 07 & 3.25 \mathrm{E} & 07\end{array}$

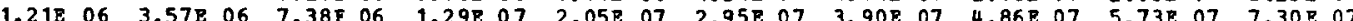

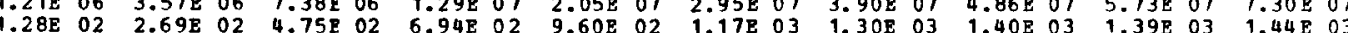
$\begin{array}{llllllllll}1.39 \mathrm{E} & 03 & 1.44 \mathrm{E} & 03 \\ \end{array}$

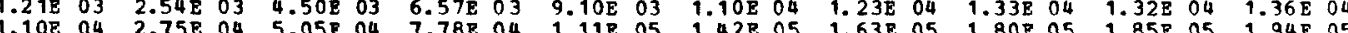

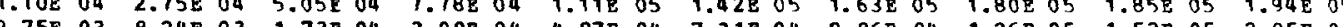

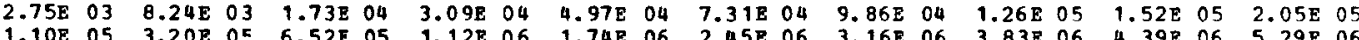

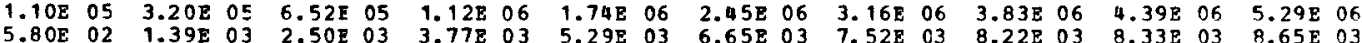

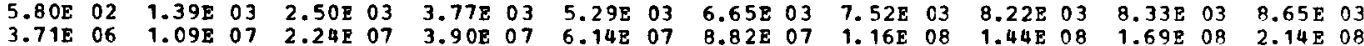

Table 3.53. Watts of heapy-element isotopes accualated at SURF (shipped 10 years after discharge) $\begin{array}{llllllllll}1990 & 1995 & 2000 & 2005 & 2010 & 2015 & 2020 & 2025 & 2030 & 2040\end{array}$

$\begin{array}{lllllllllllllllllllll}1.51 \mathrm{E} & 02 & 4.57 \mathrm{E} & 02 & 9.73 \mathrm{E} & 02 & 1.76 \mathrm{E} & 03 & 2.88 \mathrm{E} & 03 & 4.31 \mathrm{E} & 03 & 5.93 \mathrm{E} & 03 & 7.74 \mathrm{E} & 03 & 9.59 \mathrm{E} & 03 & 1.35 \mathrm{E} & 04\end{array}$

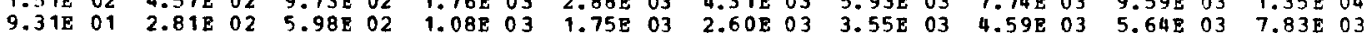

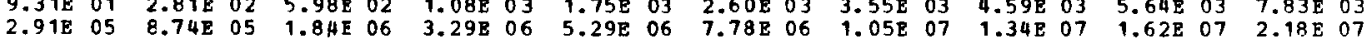

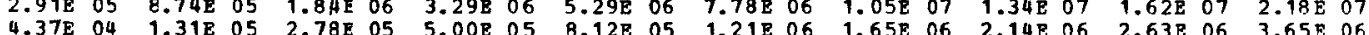

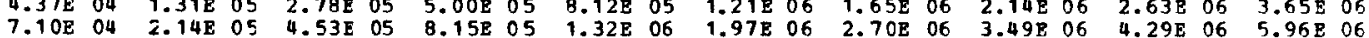

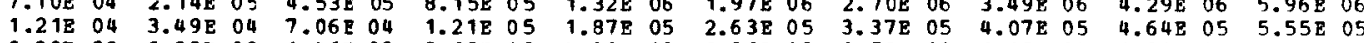
$\begin{array}{lllllllllllllllllllll}2.28 \mathrm{E} & 02 & 6.89 \mathrm{E} & 02 & 1.46 \mathrm{E} & 03 & 2.63 \mathrm{E} & 03 & 4.28 \mathrm{E} & 03 & 6.36 \mathrm{E} & 03 & 8.70 \mathrm{E} & 03 & 1.13 \mathrm{E} & 04 & 1.38 \mathrm{E} & 04 & 1.92 \mathrm{E} & 04\end{array}$ $\begin{array}{llllllllllllllllllllll}3.05 \mathrm{E} & 05 & 9.61 \mathrm{E} & 05 & 2.12 \mathrm{E} & 06 & 3.98 \mathrm{E} & 06 & 6.71 \mathrm{E} & 06 & 1.04 \mathrm{E} & 07 & 1.47 \mathrm{E} & 07 & 1.98 \mathrm{E} & 07 & 2.52 \mathrm{E} & 07 & 3.72 \mathrm{E} & 07\end{array}$ $\begin{array}{lllllllllllllllllllll}5.42 \mathrm{E} & 00 & 1.62 \mathrm{E} & 01 & 3.39 \mathrm{E} & 01 & 6.07 \mathrm{E} & 01 & 9.81 \mathrm{E} & 01 & 1.45 \mathrm{E} & 02 & 1.97 \mathrm{E} & 02 & 2.53 \mathrm{E} & 02 & 3.08 \mathrm{E} & 02 & 4.21 \mathrm{E} & 02\end{array}$ $\begin{array}{llllllllllllllllllllllll}2.51 \mathrm{E} & 03 & 7.58 \mathrm{E} & 03 & 1.51 \mathrm{E} & 04 & 2.90 \mathrm{E} & 04 & 4.71 \mathrm{E} & 04 & 7.01 \mathrm{E} & 04 & 9.58 \mathrm{E} & 04 & 1.24 \mathrm{E} & 05 & 1.52 \mathrm{E} & 05 & 2.11 \mathrm{E} & 05\end{array}$ $\begin{array}{lllllllllllllllllllll}5.77 \mathrm{E} & 02 & 1.72 \mathrm{E} & 03 & 3.61 \mathrm{E} & 03 & 6.46 \mathrm{E} & 03 & 1.04 \mathrm{E} & 04 & 1.54 \mathrm{E} & 04 & 2.10 \mathrm{E} & 04 & 2.69 \mathrm{E} & 04 & 3.28 \mathrm{E} & 04 & 4.47 \mathrm{E} & 04\end{array}$

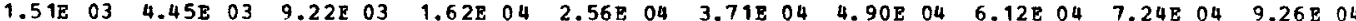

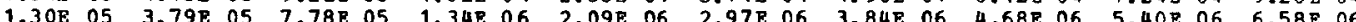
$\begin{array}{lllllllllllllllllllll}8.59 E & 05 & 2.61 E & 0 E & 5.58 E & 06 & 1.01 E & 07 & 1.65 \mathrm{E} & 07 & 2.47 \mathrm{E} & 07 & 3.40 \mathrm{E} & 07 & 4.42 \mathrm{E} & 07 & 5.45 \mathrm{E} & 07 & 7.62 \mathrm{E} & 07\end{array}$ 
Table 3.54. Ingestion toxicity ( $a^{3}$ water) of 1ight-elenent isotopes accuiviated at SURPF (shipped 10 years after discharge)

1990 1995 2000 2005 2010

2015

2020

2025

2030

2040

$\begin{array}{lrll}3 & 1.91 E & 08 \\ C & 14 & 5\end{array}$

$5.43 E$ OE $1.09 E$ Og

$\begin{array}{llll}1.84 \mathrm{E} & 099 & 2.83 \mathrm{E} & 09 \\ 9.68 \mathrm{E} & 07 & 1.57 \mathrm{E} & 08\end{array}$

$3.95 E \quad 09 \quad 5.0$

$03 \mathrm{~B} 09$

$6.02 \mathrm{E} 09$

$7.71 \mathrm{E} \quad 08$

$\begin{array}{llll}2.01 E & 09 & 3.368 & 07 \\ 2.76 E & 09\end{array}$

$5.77 E$ O9 $8.15 E$ O9

$1.04810 \quad 1.208$ O8 $1.14 \mathrm{E} 08$

CO 60

NI 59

$\begin{array}{ll}\text { NI } & 63 \\ \text { 2R } & 93\end{array}$

$\begin{array}{rl}7.57 E & 11 \\ 6.17 E & 07\end{array}$

$4.36 \mathrm{E} 11 \quad 8.53 \mathrm{E} 11$

$\begin{array}{llll}1.38 \mathrm{E} & 12 & 2.01 \mathrm{E} & 12\end{array}$

$\begin{array}{llllll}1.67 \mathrm{E} & 12 & 3.19 \mathrm{E} & 12 & 3.30 \mathrm{E} & 10\end{array}$

$1.22 \mathrm{E} 08$ 4.82E 08

$8.71 \mathrm{E} 08 \quad 1.41 \mathrm{E}$ O9 $2.10 \mathrm{E} 09$

2. $19 \mathrm{~B} 12$

$\begin{array}{ll}3.62 \mathrm{~B} & 12 \\ 3.69 \mathrm{E} & 09\end{array}$

$1.24 \mathrm{E} 06$

$3.64 \mathrm{E} \quad 06 \quad 7.54 \mathrm{E}$ 06

$\begin{array}{llllllll}7.43 \mathrm{E} & 11 & 1.19 \mathrm{E} & 12 & 1.75 \mathrm{E} & 12 & 2.36 \mathrm{E} & 12\end{array}$

$3.00 \mathrm{E} 12$

4.238 O6 $9.10 \mathrm{E}$ O6

1.698072 .208 .

$\begin{array}{llllll}3.28 \mathrm{E} & 07 & 4.50 \mathrm{E} & 07 & 5.84 \mathrm{E} & 07\end{array}$

NB 934

SN121M

$2.41 E \quad 03$

$6.99803 \quad 1.44 E$ O4

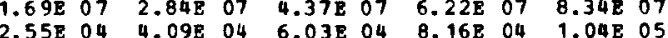

4.43809

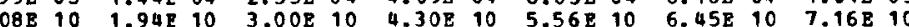

125

$\begin{array}{ll}1.84 \mathrm{E} & 09 \\ 2.27 \mathrm{E} & 11\end{array}$

$4.46 \mathrm{E}$ O5 $8.03 \mathrm{E}$ 09

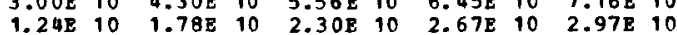

$\begin{array}{llllllllll}1.24 E & 10 & 1.78 E & 10 & 2.30 E & 10 & 2.67 E & 10 & 2.97 E & 10 \\ 2.17 E & 12 & 3.28 E & 12 & 4.52 E & 12 & 5.66 E & 12 & 6.75 E & 12\end{array}$

$\begin{array}{rlll}6.798 & 09 & 7.98 \mathrm{E} & 09\end{array}$

$\begin{array}{llll}5.09 \mathrm{E} & 08 & 7.07 \mathrm{E} & 08\end{array}$

$1.34 E \quad 10 \quad 1.38 E \quad 10$

$3.86 \mathrm{E} 12$ 4. $14 \mathrm{E} \quad 12$

$\begin{array}{lllll}4.53 \mathrm{E} & 09 & 6.26 \mathrm{E} & 09\end{array}$

$3.63 \mathrm{E} \quad 12 \quad 4.87 \mathrm{E} 12$

$7.19 \mathrm{E} 07 \quad 1.00 \mathrm{E} 08$

$1.26 \mathrm{E}$ 05 $1.70 \mathrm{E}$ 05

$7.35 \mathrm{E} \quad 10 \quad 7.78 \mathrm{E} 10$

$3.04 \mathrm{E} 10 \quad 3.22 \mathrm{E} 10$

$\begin{array}{llll}3.04 \mathrm{E} & 10 & 3.22 \mathrm{E} & 10 \\ 7.63 \mathrm{E} & 12 & 9.15 \mathrm{E} & 12\end{array}$

Table 3.55. Ingestion toxicity $\left(\mathrm{n}^{3}\right.$ water) of fission-product isotopes accunulated at SURF (shipped 10 years after discharge)

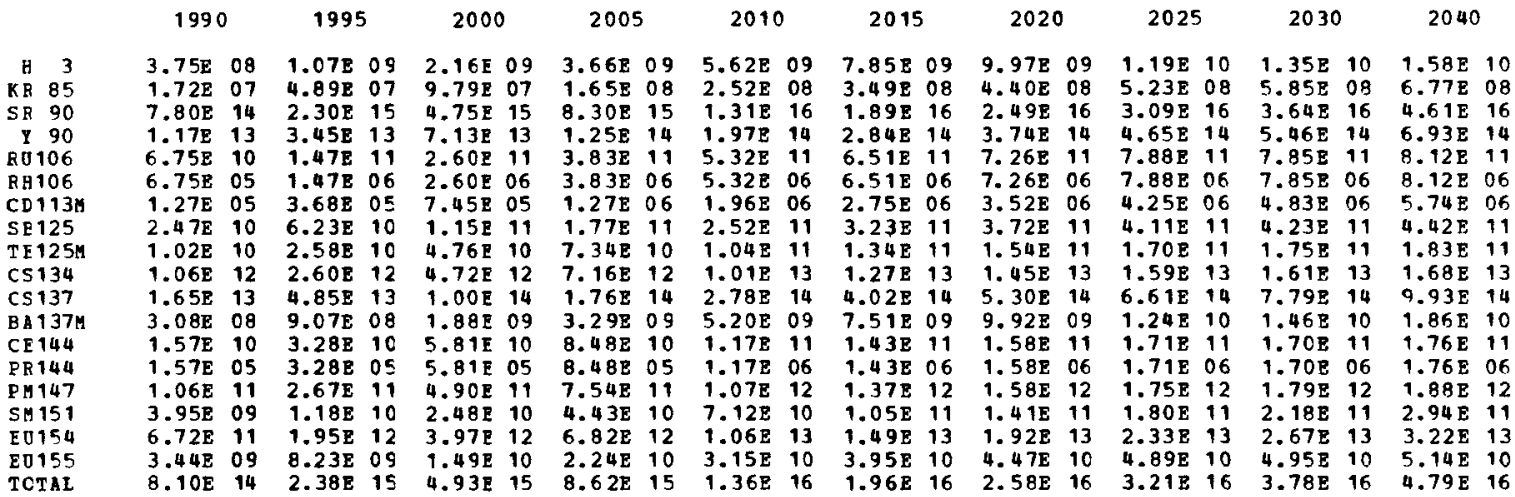

Table 3.56. Ingestion toxicity ( $\mathrm{m}^{3}$ water) of heavy-element isotopes accuiviated at SoRP (shipped 10 years after discharge)

$\begin{array}{llllllllll}1990 & 1995 & 2000 & 2005 & 2010 & 2015 & 2020 & 2025 & 2030 & 2040\end{array}$

0234

NP 239

P0238

PU 239

$\mathrm{P} 0240$

PU241

$P 0242$

A 12424

A 243

CM242

C. 243

Cत 243

TOTAL

\begin{tabular}{|c|c|c|c|c|c|c|c|c|c|c|c|c|c|c|c|c|c|c|}
\hline $\begin{array}{l}75 E \\
89 E \\
76 E \\
81 E \\
56 E \\
46 E\end{array}$ & $\begin{array}{l}08 \\
08 \\
12 \\
11 \\
11 \\
12\end{array}$ & $\begin{array}{l}5.29 \mathrm{E} \\
2.08 \mathrm{E} \\
5.28 \mathrm{E} \\
8.45 \mathrm{E} \\
1.37 \mathrm{E} \\
4.21 \mathrm{E}\end{array}$ & $\begin{array}{l}08 \\
09 \\
12 \\
11 \\
12 \\
12\end{array}$ & $\begin{array}{l}1.13 \mathrm{E} \\
4.42 \mathrm{E} \\
1.11 \mathrm{E} \\
1.79 \mathrm{E} \\
2.91 \mathrm{E} \\
8.51 \mathrm{E}\end{array}$ & $\begin{array}{l}09 \\
09 \\
13 \\
12 \\
12 \\
12\end{array}$ & $\begin{array}{l}2.04 \mathrm{E} \\
7.96 \mathrm{E} \\
1.99 \mathrm{E} \\
3.21 \mathrm{E} \\
5.23 \mathrm{E} \\
1.46 \mathrm{E}\end{array}$ & $\begin{array}{l}09 \\
09 \\
13 \\
12 \\
12 \\
13\end{array}$ & $\begin{array}{l}3.33 \mathrm{E} \\
1.29 \mathrm{E} \\
3.19 \mathrm{E} \\
5.22 \mathrm{E} \\
8.50 \mathrm{E} \\
2.25 \mathrm{E}\end{array}$ & $\begin{array}{l}09 \\
10 \\
13 \\
12 \\
12 \\
13\end{array}$ & $\begin{array}{l}4.99 \mathrm{E} \\
1.92 \mathrm{E} \\
4.70 \mathrm{E} \\
7.77 \mathrm{E} \\
1.26 \mathrm{E} \\
3.17 \mathrm{E}\end{array}$ & $\begin{array}{l}09 \\
10 \\
13 \\
12 \\
13 \\
13\end{array}$ & $\begin{array}{l}6.87 \mathrm{E} \\
2.63 \mathrm{E} \\
6.34 \mathrm{E} \\
1.06 \mathrm{E} \\
1.73 \mathrm{E} \\
4.06 \mathrm{E}\end{array}$ & $\begin{array}{l}09 \\
10 \\
13 \\
13 \\
13 \\
13\end{array}$ & $\begin{array}{l}8.96 \mathrm{E} \\
3.40 \mathrm{E} \\
8.09 \mathrm{E} \\
1.38 \mathrm{E} \\
2.24 \mathrm{E} \\
4.91 \mathrm{E}\end{array}$ & $\begin{array}{l}09 \\
10 \\
13 \\
13 \\
13 \\
13\end{array}$ & $\begin{array}{l}1.11 \mathrm{E} \\
4.17 \mathrm{E} \\
9.80 \mathrm{E} \\
1.69 \mathrm{E} \\
2.75 \mathrm{E} \\
5.59 \mathrm{E}\end{array}$ & $\begin{array}{l}10 \\
10 \\
13 \\
13 \\
13 \\
13\end{array}$ & $\begin{array}{l}1.57 \mathrm{E} \\
5.79 \mathrm{E} \\
1.32 \mathrm{E} \\
2.35 \mathrm{E} \\
3.92 \mathrm{E} \\
6.69 \mathrm{E}\end{array}$ \\
\hline & $\begin{array}{l}09 \\
12\end{array}$ & & $\begin{array}{l}05 \\
12\end{array}$ & & $\begin{array}{l}09 \\
13\end{array}$ & & $\begin{array}{l}10 \\
13\end{array}$ & & $\begin{array}{l}10 \\
13\end{array}$ & & $\begin{array}{l}10 \\
13\end{array}$ & & $\begin{array}{l}10 \\
14\end{array}$ & & & & $\begin{array}{l}10 \\
14\end{array}$ & \\
\hline & 09 & & & & 10 & & 10 & & 10 & & 11 & & 11 & $2 \mathrm{E}$ & 11 & & 11 & \\
\hline & 10 & & 10 & & 11 & & 11 & & 11 & & 11 & & 11 & & & & & \\
\hline & 08 & & os & & 09 & & 09 & & 10 & & 10 & & 10 & & & & 10 & \\
\hline & 09 & & $1 \mathrm{C}$ & & 10 & & 10 & & 11 & & 11 & & 1 & & & & 1 & \\
\hline & 11 & & 12 & & 12 & & 12 & & 12 & & 13 & & 13 & & & & 3 & \\
\hline & 12 & & 13 & $.36 \mathrm{E}$ & 13 & $5 \mathrm{E}$ & 13 & $.28 \mathrm{E}$ & 14 & $90 \mathrm{E}$ & 14 & & 14 & & 94 & $4.11 \mathrm{~B}$ & 14 & \\
\hline
\end{tabular}

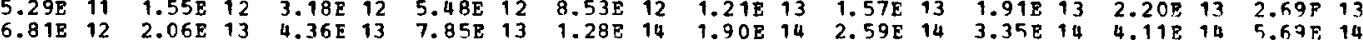


Table 3.57. Inhalation toxicity ( $\mathrm{a}^{3}$ air) of light-elenent isotopes accunulated at SORPP (shipped 10 years after discharge)

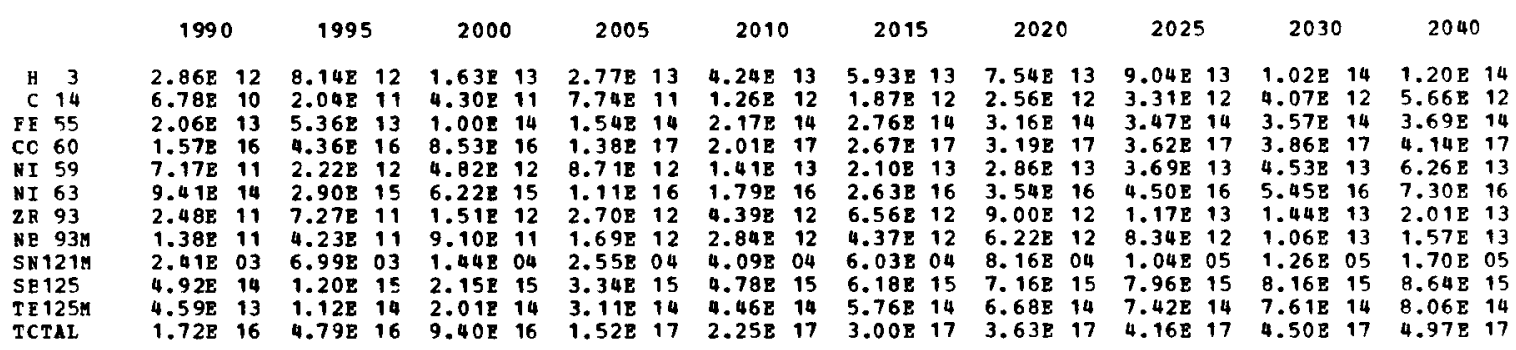

Table 3.58. Inhalation toricity ( $\mathbf{m}^{3}$ air) of fission-product isotopes accumulated at SURF (shipped 10 years after discharge)

\begin{tabular}{|c|c|c|c|c|c|c|c|c|c|c|c|c|c|c|c|c|c|c|c|c|}
\hline & \multicolumn{2}{|c|}{1990} & \multicolumn{2}{|c|}{1995} & \multicolumn{2}{|l|}{2000} & \multicolumn{2}{|c|}{2005} & \multicolumn{2}{|l|}{2010} & \multicolumn{2}{|c|}{2015} & \multicolumn{2}{|c|}{2020} & \multicolumn{2}{|l|}{2025} & \multicolumn{2}{|l|}{2030} & \multicolumn{2}{|l|}{2040} \\
\hline H 3 & $5.62 \mathrm{E}$ & 12 & $61 \mathrm{E}$ & 13 & $.24 \mathrm{E}$ & 13 & 3.5 & 13 & $8.43 \mathrm{~B}$ & 13 & $1.18 \mathrm{E}$ & 14 & $1.49 E$ & 14 & $1.79 \mathrm{E}$ & 1 & $2.02 \mathrm{E}$ & 14 & $2.37 \mathrm{E}$ & \\
\hline CR 85 & & 13 & & 14 & & 14 & & & & 14 & & & & & & & & & & \\
\hline 90 & & 18 & & 19 & & 19 & & 19 & & 20 & & 20 & $2.49 \mathrm{E}$ & 2 & $9 \mathrm{E}$ & 0 & & 20 & & \\
\hline 90 & & 16 & & 17 & & 17 & & 17 & & 18 & & & & & & & & & & \\
\hline 106 & & 15 & & 15 & & 16 & & 16 & & 1 & & & & & & & & 6 & & \\
\hline 106 & & 05 & 1.1 & 06 & & 06 & & 06 & & 05 & & & & 0 & & & & & $2 E$ & \\
\hline 113 & $\begin{array}{l}1.27 \mathrm{E} \\
2.74 \mathrm{E}\end{array}$ & $\begin{array}{l}05 \\
15\end{array}$ & $\begin{array}{l}3.6 \\
6.9\end{array}$ & $\begin{array}{l}05 \\
15\end{array}$ & & $\begin{array}{l}05 \\
16\end{array}$ & & $\begin{array}{l}06 \\
16\end{array}$ & & $\begin{array}{l}06 \\
16\end{array}$ & & & & $\begin{array}{l}0 \\
1\end{array}$ & & & & & & \\
\hline 12 & $2.55 \mathrm{E}$ & 14 & $6.46 \mathrm{E}$ & 14 & 198 & 15 & & 15 & & 15 & & 1 & & 1 & & & & 5 & & \\
\hline & & 16 & & 16 & & 17 & & 1 & & 1 & & & & 1 & & & & 17 & & \\
\hline 137 & & 17 & & 18 & & 18 & & 1 & & 1 & & & & & & & & 19 & & \\
\hline 13 & $8 E$ & 08 & 78 & $O E$ & $1.88 \mathrm{E}$ & 09 & & 09 & & 09 & & 0 & & 0 & $1.24 \mathrm{E}$ & & & 10 & & \\
\hline & & 14 & & 15 & & 15 & & 15 & & 15 & & & & & & & & 5 & & \\
\hline & & 05 & & 05 & & 05 & & & & 06 & & & & & & & & & & \\
\hline & & 16 & & 16 & & 16 & & 18 & & 17 & & 17 & & 1 & & & & 17 & & \\
\hline & & 14 & & 15 & & & & & & 16 & & & & & & & & & & \\
\hline & & $\begin{array}{l}17 \\
14\end{array}$ & & $\begin{array}{l}17 \\
14\end{array}$ & & $\begin{array}{l}17 \\
14\end{array}$ & & $\begin{array}{l}18 \\
15\end{array}$ & & $\begin{array}{l}1 \\
1\end{array}$ & & 1 & & & $\begin{array}{l}7 \mathrm{E} \\
\mathrm{SE}\end{array}$ & & & $\begin{array}{l}18 \\
15\end{array}$ & & \\
\hline TAL & & 18 & $2.56 \mathrm{E}$ & 19 & $5.30 \mathrm{E}$ & 19 & & 19 & $1.46 \mathrm{~B}$ & 20 & & 20 & $2.77 \mathrm{E}$ & 20 & $3.44 \mathrm{E}$ & & $4.05 \mathrm{E}$ & 20 & $5.13 \mathrm{E}$ & \\
\hline
\end{tabular}

Iable 3.59. Inhalation toxicity ( $n^{3}$ air) of heary-elenent isotopes accunulated at SURPF (shipped 10 years after discharge)

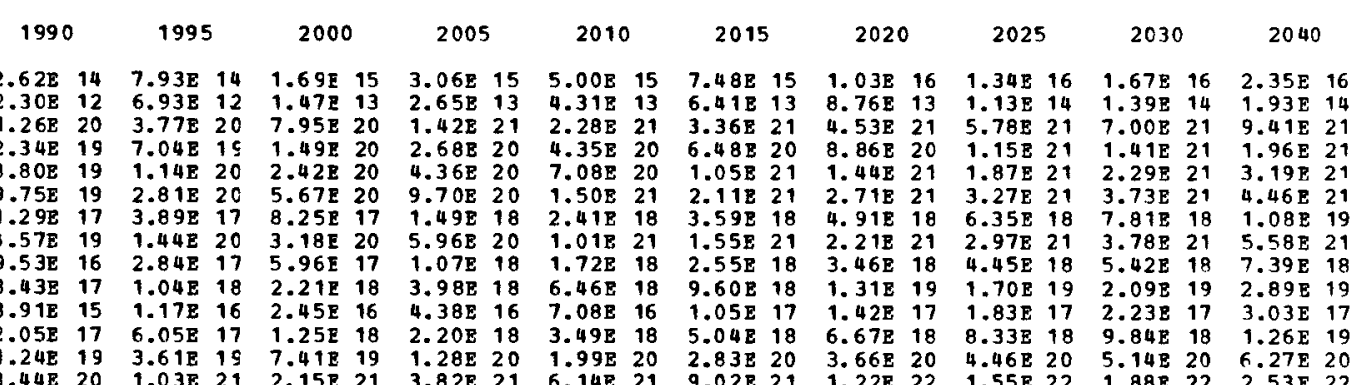


Table 3.60. Curies of accunulated light-eleant isotopes after 2041

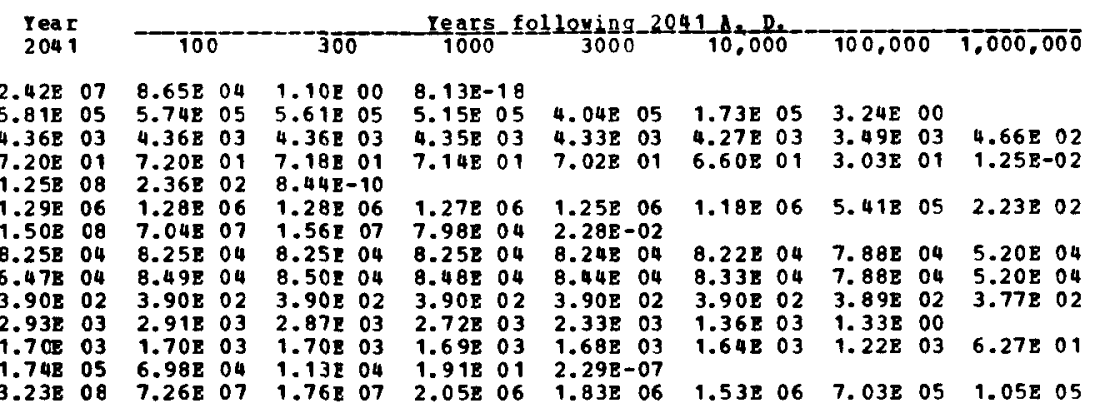

Table 3.61. Curies of accuaulated fission-product isotopes after 2041

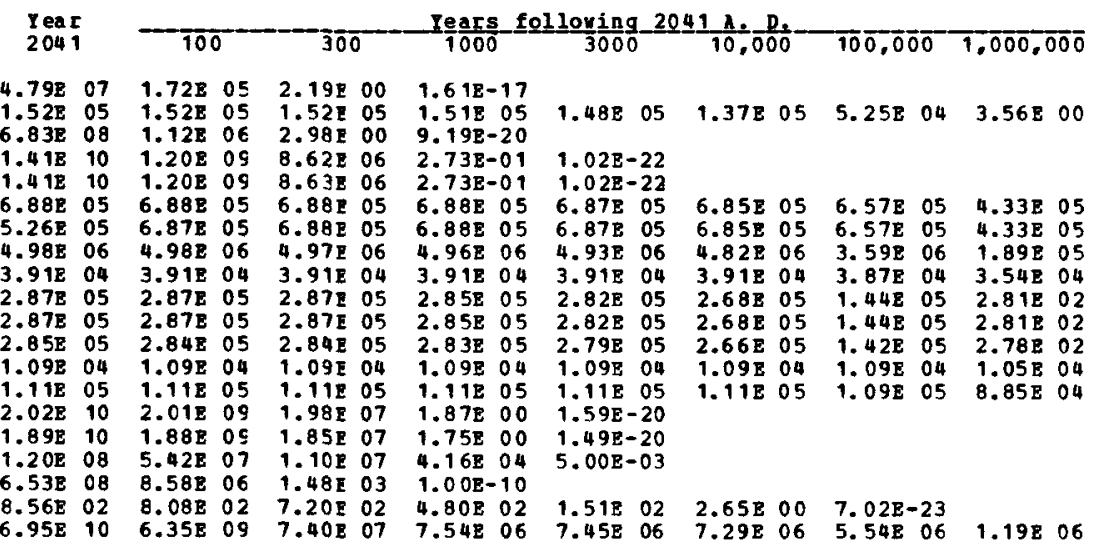


Table 3.62. Carles of accunlated hesvy-elenent 1sotopes after 2041

rear 2041 100 300

$5.44 E 00$ 6.58E-02

3.38z-01

5.468 00

1.02800

$3.39 \mathrm{E}-01$ $6.58 \mathrm{E}-02$

$6.588-02$
1.02800

3.22E-01

$6.44 z-02$

$1.02 E 00$

$5.46 \mathrm{E} 00$

1.02800

$6.588-02$
$5.46 \mathrm{E}$

1.02200

$6.58 \mathrm{E}-02$

5.46800

$6.58 \mathrm{E}-02$
$1.02 \mathrm{E} 00$

$6.58 \mathrm{-c2}$

$5.44 E 00$

$5.38 \mathrm{E} 00$

$6.578-02$

7.89203

1.328 o5

$1.22 E 05$

1.32805

2.30E 01

4.84E 05

$\begin{array}{lll}7.898 & 03 \\ 9.428 & 04\end{array}$

$\begin{array}{lll}9.428 & 04 \\ 1.32 E & 05\end{array}$

1. 22805

$5.95 \mathrm{E} 06$

$6.74 E 08$

$1.21 E 08$

1.97E $O B$

$1.35 \mathrm{E} 10$

6.698
1.158

$1.52 \mathrm{E} 06$

1.52806

5.95E 06

$\begin{array}{ll}1.25 E & 06 \\ 2.57 E & 06\end{array}$

2.57E 08

$1.59 \mathrm{E} 10$
5.58201

8.181-02

3.722 00

5.60101

1.14202

9.1420101

9.4018
5.608
3.72

3.72800

9.14202

9.40201

3.64800

1.14202
5.608

1.14102

3.72800

5.608 or

1.14202

5.60201

3.72100

1.14802

3.72100

5.608 or

5.52101

3.721 o0 1.77E 03 1.328 o5

6.12201

2.44205

1.32105

2.628 02

7.93803

9.93803

$1.32 \mathrm{E} 05$

2.44205

5.79806

6.57807

1.20208

$1.91 \mathrm{E}$ O8

$\begin{array}{lll}1.642 & 04 \\ 6.691 & 05\end{array}$

1.012 og

3.862 05

$3.86 \mathrm{P} 05$

5.79806

$\begin{array}{llll}3.172 & 05 \\ 3.878 & 03\end{array}$

1.95203

1.40209 rears

1000

$\frac{94 i n g}{3000}$

10,000

$.78 E 02 \quad 5.098$ o2

$4.98801 \quad 5.72 \mathrm{E} 02$

$1.15803 \quad 0.14803$

1.785 O2 5.10802

$1.15 E 03$ B.14E 03

1.158038 .14803

$1.78 \mathrm{E} 02 \quad 5.10 \mathrm{z} 02$

$\begin{array}{llll}4.982 & 01 & 5.722 & 02 \\ 1.158 & 03 & 8.148 & 03\end{array}$

$\begin{array}{llll}1.158 & 03 & 8.148 & 03 \\ 1.158 & 03 & 8.148 & 03\end{array}$

4.872 or 5.608 o

1.158 O3 $8.14 \mathrm{~B} 03$

1.788025 .10802

$1.15 \mathrm{E} 03 \quad 8.14 \mathrm{E} 03$

$4.98 \mathrm{~B} 01 \quad 5.72 \mathrm{E} 02$

$1.78 \mathrm{E} 025.10 \mathrm{E} \quad 02$

4.988 o1 $5.72 \mathrm{E}$ O2

$1.78802 \quad 5.10802$

$4.98 \mathrm{E}$ or $5.72 \mathrm{E} 02$

$1.15 \mathrm{E} 03 \quad 8.14 \mathrm{~B} 03$

$4.98 \mathrm{E}$ o1 $5.72 \mathrm{E}$ O2

1.788 O2 $5.10 \mathrm{E} 02$

$1.76 \mathrm{E} 02 \quad 5.03 \mathrm{E} 02$

$4.98 \mathrm{E} 01$ 5.72E 02

$\begin{array}{llll}6.13 \mathrm{~B} & 03 & 1.85 \mathrm{E} & 04 \\ 8.018 & 03 & 8.23 \mathrm{E} & 03\end{array}$

$1.32 E$ OS $1.32 E$ OS

1.788025 .10802

$3.81 \mathrm{E}$ 05 4.44E 05

1.32E 05 1.32E 05

$7.26 \mathrm{~B}$ 05 $7.23 \mathrm{E}$ 05

$\begin{array}{llll}7.268 & 05 & 7.23 E & 05 \\ 8.018 & 03 & 8.23 E & 03\end{array}$

$\begin{array}{llll}8.018 & 03 & 8.238 & 03 \\ 9.968 & 04 & 1.098 & 05\end{array}$

1. $32 E$ O5 $1.32 E$ 05

$3.81 \mathrm{E} 05 \quad 4.44 \mathrm{E} 05$

$\begin{array}{llll}5.438 & 06 & 4.538 & 06 \\ 3.108 & 05 & 3.498 & 00\end{array}$

1.18E OB 1.11E OO

1.78E OB 1.45E 08

$7.12803 \quad 6.02 \mathrm{E} 03$

$\begin{array}{llll}6.688 & 05 & 6.65 \mathrm{E} & 05 \\ 3.288 & 08 & 1.338 & 07\end{array}$

1.598 O4 1.748 00

i. $59 \mathrm{E}$ O4 $1.74 \mathrm{E}$ OO

$\begin{array}{llll}1.598 & 04 & 1.74 \mathrm{E} & 00 \\ 5.43 \mathrm{E} & 06 & 4.53 \mathrm{E} & 06\end{array}$

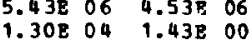

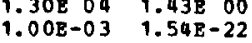

$2.318-04 \quad 2.318-04 \quad 2.318-04 \quad 2.318-04 \quad 2.298-04$

6.378 $08 \quad 2.82 \mathrm{E} \quad 08$ $\begin{array}{lllllll}.638 & 03 & 9.888 & 03 & 1.218 & 04\end{array}$ $\begin{array}{llllll} & & \end{array}$ $\begin{array}{llllll}198 & 03 & 1.398 & 05 & 3.448 & 05\end{array}$ $\begin{array}{llllllll}1.64 E & 04 & 3.74 E & 05 & 1.852 & 05\end{array}$

$\begin{array}{lllllll}.63 E & 03 & 9.918 & 03 & 1.218 & 04\end{array}$ $.64204 \quad 3.74805 \quad 1.85805$ $\begin{array}{lllll}9.918 & 03 & 1.218 & 04\end{array}$ .19E 03 1.39E 05 3.44E 05 4.6 04 3.74E 05 1.85E O5 6.65E 0U 3.748 05 1.85E 05 1.36E O5 3.37E 05 .65E O4 3.74E 05 1.85B 05 $\begin{array}{lllllll}.638 & 03 & 9.912 & 03 & 1.218 & 04\end{array}$ $.64 \mathrm{E}$ O4 3.74E 05 1.85E 05 $\begin{array}{llllll}198 & 03 & 1.39 \mathrm{E} & 05 & 3.44 \mathrm{~B} & 05\end{array}$ $\begin{array}{lllllll}1.63 E & 03 & 9.91 E & 03 & 1.21 E & 04\end{array}$ $\begin{array}{llllllll}6.648 & 04 & 3.74 \mathrm{E} & 05 & 1.85 \mathrm{~B} & 05\end{array}$ $19 \mathrm{E} 03 \quad 1.39 \mathrm{E} 05 \quad 3.44 \mathrm{E} 05$ $1.63 \mathrm{E} 03 \quad 9.91 \mathrm{E} 03 \quad 1.21804$ $6.19 \mathrm{E} 03 \quad 139 \mathrm{~B} 05 \quad 3.44805$ 648 04 3.74 051.85805 $\begin{array}{llllll}198 & 1.398 & 05 & 3.44 E & 05\end{array}$ $.63 \mathrm{E} 03 \quad 9.91803 \quad 1.21804$ $\begin{array}{llllll}1.618 & 03 & 9.77 \mathrm{E} & 03 & 1.198 & 04\end{array}$ $\begin{array}{lllllll}6.198 & 03 & 1.39 E & 05 & 3.448 & 05\end{array}$ $\begin{array}{llllll}5.96 \mathrm{E} & 04 & 3.72 \mathrm{E} & 05 & 1.85 \mathrm{E} & 05\end{array}$ $\begin{array}{llllll}9.92 E & 03 & 1.198 & 04 & 1.21 E & 04\end{array}$ $\begin{array}{llllll}.32 \mathrm{E} & 05 & 1.32 \mathrm{E} & 05 & 1.32 \mathrm{E} & 05\end{array}$ $\begin{array}{llllll}.63 \mathrm{E} & 03 & 9.91 \mathrm{E} & 03 & 1.218 & 04\end{array}$ $\begin{array}{lllllll}468 & 05 & 4.33 \mathrm{E} & 05 & 3.24 \mathrm{E} & 05\end{array}$ $\begin{array}{llllll}1.32 \mathrm{E} & 05 & 1.32 \mathrm{E} & 05 & 1.32 \mathrm{E} & 05\end{array}$ 1.798 O4 $1.53 E 05 \quad 3.44805$ 1. $12=0505$ T.68 05 1.198 O4 1.21804 1. 198 OS 1.218 O4 1.30 05 1.46E 05 . 3605 1. 325 O5 $\begin{array}{lllll}.40 \mathrm{E} & 06 & 6.91 \mathrm{E} & 02 & 1.13 \mathrm{E}-02\end{array}$ $4.71 E-14$

$9.19 E$ of $7.21 E$ 06 1.13E-02 $\begin{array}{lllll}.078 & 07 & 6.94 \mathrm{E} & 03 & 1.76 \mathrm{E}-01\end{array}$ $\begin{array}{llll}.35 E & 03 & 1.778 & 00\end{array}$ $\begin{array}{lllllll}.57 \mathrm{E} & 05 & 5.57 \mathrm{E} & 05 & 1.07 \mathrm{E} & 05\end{array}$ $\begin{array}{llll}5.77 \mathrm{E} & 00\end{array}$

$38 E-14$

2. $38 E-14$

$.40 \mathrm{E} 06 \quad 6.87 \mathrm{~B} \quad 02 \quad 1.13 \mathrm{E}-02$ $1.71 E$ OB $1.49 E$ O7 $6.54 \mathrm{E} 06$
$.64 \mathrm{E} 04 \quad 3.74 \mathrm{E} 05 \quad 1.85 \mathrm{E} 05$ 
Table 4.1. NAC projections of spent fuel discharged ${ }^{a}$

\begin{tabular}{|c|c|c|c|c|c|c|c|c|c|}
\hline \multirow[b]{2}{*}{ Year } & \multirow{2}{*}{$\begin{array}{l}\text { Installed } \\
\text { capacity } \\
\text { (MW) }\end{array}$} & \multicolumn{3}{|c|}{$\begin{array}{c}\text { Number of assemblies } \\
\text { discharged per year }\end{array}$} & \multicolumn{3}{|c|}{$\begin{array}{l}\text { Metric tons of heavy } \\
\text { metal discharged/year }\end{array}$} & \multicolumn{2}{|c|}{ Accumulations $^{\mathbf{a}}$} \\
\hline & & BWR & PWR & Total & BWR & PWR & Total & $\begin{array}{l}\text { Number of } \\
\text { assemblies }\end{array}$ & $\overline{\text { MTHM }^{b}}$ \\
\hline 1976 & 40,108 & & & 2,705 & & & 772 & 7,490 & 1,925 \\
\hline 1977 & 49,796 & 3,025 & 1,346 & 4,371 & 510 & 524 & 1,034 & 11,861 & 2,959 \\
\hline 1978 & 54,671 & 3,142 & 1,973 & 5,115 & 562 & 795 & 1,357 & 16,976 & 4,316 \\
\hline 1979 & 63,622 & 3,117 & 2,361 & 5,478 & 555 & 970 & 1,525 & 22,454 & 5,841 \\
\hline 1980 & 75,936 & 3,763 & 2,924 & 6,687 & 656 & 1,207 & 1,863 & 29,141 & 7,704 \\
\hline 1981 & 89,691 & 3,679 & 3,028 & 6,707 & 640 & 1,256 & 1,896 & 35,848 & 9,600 \\
\hline 1982 & 106,739 & 5,161 & 3,725 & 8,886 & 922 & 1,551 & 2,473 & 44,734 & 12,073 \\
\hline 1983 & 121,883 & 5,551 & 4,509 & 10,060 & 976 & 1,900 & 2,876 & 54,794 & 14,949 \\
\hline 1984 & 143,874 & 6,283 & 5,108 & 11,391 & 1,103 & 2,137 & 3,240 & 66,185 & 18,189 \\
\hline 1985 & 158,721 & 8,489 & 5,936 & 14,425 & 1,494 & 2,487 & 3,981 & 80,610 & 22,170 \\
\hline 1986 & 175,851 & 9,261 & 6,420 & 15,681 & 1,629 & 2,687 & 4,316 & 96,291 & 26,486 \\
\hline
\end{tabular}

a Does not include 417 metric tons of heavy metal already in away-from-reactor storage and 244 metric tons of heavy metal already reprocessed.

${ }^{b}$ Metric tons of heavy metal. 
ORNL/ TM-6008

\section{INTERNAL DISTRIBUTION}

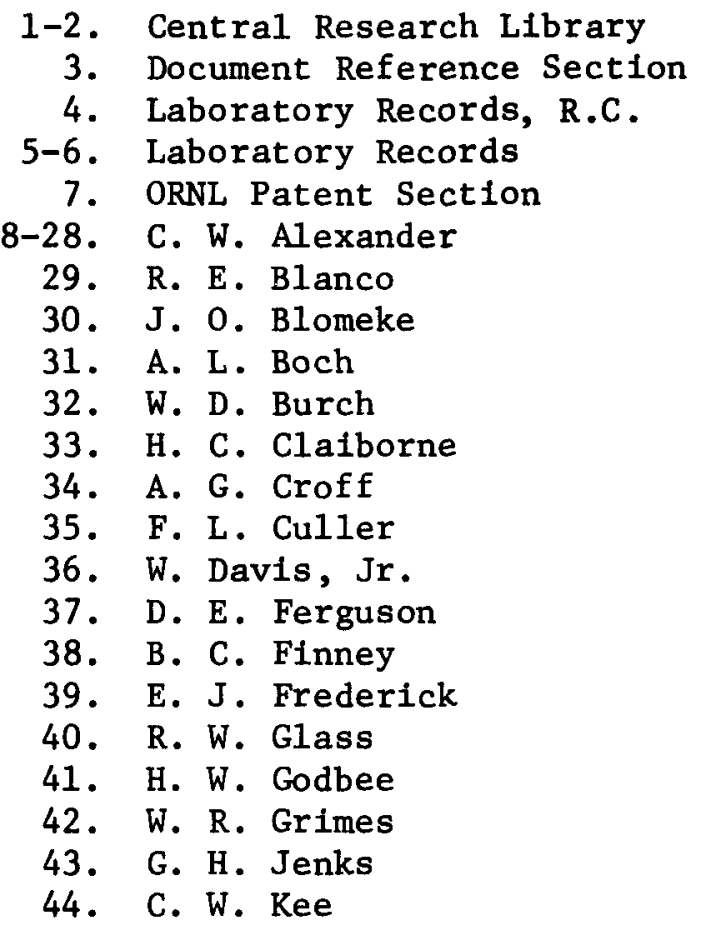

45. E. Lamb

46. R. E. Leuze

47. K. H. Lin

48. W. C. McClain

49. J. D. McGaugh

50. L. E. McNeese

51. J. G. Moore

52. J. M. Morrison

53. K. J. Notz

54. C. D. Scott

55. D. W. Tedder

56. D. B. Trauger

57. D. W. Turner

58. B. L. Vondra

59. R. G. Wymer

60. G. R. Choppin (consultant)

61. E. L. Gaden, Jr. (consultant)

62. C. H. Ice (consultant)

63. L. E. Swabb, Jr. (consultant)

64. K. D. Timmerhaus (consultant)

EXTERNAL DISTRIBUTION

65-91. Technical Information Center, Oak Ridge, TN 37830

DOE/ORO, Oak Ridge, TN 37830

92. Research and Technical Support Division

93. J. J. Schreiber

Department of Energy, Washington, DC 20545

94. C. B. Bastin

95. C. R. Cooley

96. W. K. Eister

97. J. A. Leary

U.S. Nuclear Regulatory Commission, Washington, DC 20555

98. C. B. Bartlett

99. W. P. Bishop

DOE/Nevada Operations Office, P. O. Box 14100, Las Vegas, NV 89114

100. R. M. Nach

101. D. T. Schueler

102. J. W. Henderson 
DOE/Savannah River Operations, P. O. Box A, Alken, SC 29801 103. W. J. Brumbey

E. I. du Pont de Nemours and Company, Aiken, SC 29801 104. S. D. King

Battelle Northwest, P. 0. Box 999, Richland, WA 99352 105. R. M. Fleischman 106. R. C. Lilkala

107. A. M. Platt

108. R. D. Widrig

Exxon Nuclear, 2101 Horn Rapids Road, Richland, WA 99352 109. L. T. Lakey

Allied-General Nuclear Services, P. O. Box 847, Barnwe11, SC 29812 110. A. K. Williams

Argonne National Laboratory, 9700 South Cass Ave., Argonne, IL 62439 111. M. J. Steindler

Hanford Engineering Development Laboratory, P. O. Box 1970, Richland, WA 99352

112. R. L. Knecht

Rockwell Hanford Operations, P. 0. Box 800, Richland, WA 99352 113. W. J. Kurzeka

114. D. A. Turner

DOE/Richland Operations Office, P. O. Box 550, Richland, WA 99352 115. R. B. Goranson

116. S. L. Goldsberry

Sandia Laboratories, P. O. Box 5800, Albuquerque, NM 87115

117. P. D. O'Brien

118. J. Campbe11

Nuclear Fuel Services, Inc., P. 0. Box 124, West Valley, NY 14171 119. J. P. Duckworth

The University of Arizona, Department of Nuclear Engineering, Tucson, AZ 85721

120. R. G. Post

Science Applications, Inc., 1600 Anderson Road, McLean, VA 22101 121. G. Bray

Allied Chemical Corp., P. O. Box 2204, Idaho Falls, ID 83401 122. B. C. Musgrave 
George C. Marshall Space Flight Center, Marshall Space Flight Center, AL 35812

123. R. E. Burns

The University of California, Department of Nuclear Engineering, Berkeley, CA 94720

124. T. H. Pigford

General Electric Company, 175 Curtner Ave., San Jose, CA 95100 125. A. B. Carson

Georgia Institute of Technology, Department of Nuclear Engineering, Atlanta, GA 30332

126. Alfred Schneider

Nuclear Safety Associates, 5101 River Road, Bethesda, MD 20016 127. W. A. Rodger

E. R. Johnson Associates, 8206 Leesburg Pike, Vienna, VA 22180 128. J. A. McBride

Westinghouse Electric Corp., P. O. Box 355, Pittsburgh, PA 15230 129. A. Rosenblatt

Jet Propulsion Laboratory, California Institute of Technology, 4800 Oak Grove Drive, Pasadena, CA 91103

130. Ed Edelson

North Carolina State University, P. 0. Box 5636, Nuclear Engineering Department, Rale1gh, NC 27607

131. Lloyd Zumwalt 